Published in final edited form as:

Cochrane Database Syst Rev. ; (3): CD007949. doi:10.1002/14651858.CD007949.

\title{
Addition of long-acting beta-agonists to inhaled corticosteroids for chronic asthma in children
}

\author{
Muireann Ni Chroinin ${ }^{1}$, Toby J Lasserson², llana Greenstone ${ }^{3}$, and Francine M Ducharme ${ }^{4}$ \\ ${ }^{1}$ Division of Children's Services, Cork University Hospital, Cork, Ireland ${ }^{2}$ Community Health \\ Sciences, St George's, University of London, London, UK ${ }^{3}$ Montreal Children's Hospital, \\ Montreal, Canada ${ }^{4}$ Research Centre, CHU Sainte-Justine and the Department of Pediatrics, \\ University of Montreal, Montreal, Canada
}

\begin{abstract}
Background-Long-acting $ß_{2}$-agonists (LABA) in combination with inhaled corticosteroids (ICS) are increasingly prescribed in asthmatic children.

Objectives-To compare the safety and benefit of adding LABA to ICS with the same or an increased dose of ICS in children with persistent asthma.

Search methods-We searched the Cochrane Airways Group Asthma Trials Register (May 2008).

Selection criteria-We included randomised controlled trials testing the combination of LABA and ICS versus the same or an increased dose of ICS for minimum of at least 28 days in children and adolescents with asthma. The main outcome was the rate of exacerbations requiring rescue oral steroids. Secondary outcomes included pulmonary function, symptoms, adverse events, and withdrawals.
\end{abstract}

Data collection and analysis-Studies were assessed independently by two review authors for methodological quality and data extraction. Confirmation was obtained from the trialists when possible.

Copyright (C) 2010 The Cochrane Collaboration. Published by John Wiley \& Sons, Ltd.

Contact address: Muireann Ni Chroinin, Division of Children's Services, Cork University Hospital, Cork, Ireland.

Muireann.NiChroinin@hse.ie.

Editorial group: Cochrane Airways Group.

Publication status and date: Edited (no change to conclusions), published in Issue 2, 2010.

Review content assessed as up-to-date: 8 December 2008.

CONTRIBUTIONS OF AUTHORS

MNC: Protocol initiation \& write-up; study assessment, characterisation \& data extraction; write-up

FD: Protocol development; review development and write-up

TJL: Study assessment, data extraction; analysis; write-up

IG: Protocol development, write-up

DECLARATIONS OF INTEREST

In the past five years, Francine Ducharme received some research funding from GSK, MERCK, and Astra Zeneca and gave CME conferences supported by Merck Frost. M Ni Chroinin has received some research funding and given a CME lecture sponsored by Astra Zeneca and attended CME conferences supported by GSK. Toby Lasserson, Ilana Greenstone and previous authors: A Danish, $\mathrm{H}$ Magalinos, V Masse and X Zhang report no conflict of interest. 
Main results-A total of 25 trials representing 31 control-intervention comparisons were included in the review randomising 5572 children. Most of the participants were inadequately controlled on current ICS dose. We assessed the addition of LABA to the same dose of ICS and to an increased dose of ICS:

(1) The addition of LABA to ICS was compared to same dose ICS, namely $400 \mathrm{mcg} / \mathrm{day}$ of beclomethasone or less in 16 of the 24 studies. The mean age of participants was 10 years and males accounted for $64 \%$ of the study populations. The mean $\mathrm{FEV}_{1}$ at baseline was $80 \%$ of predicted or above in 10 studies; $\mathrm{FEV}_{1} 61 \%$ to $79 \%$ of predicted in eight studies; and unreported in the remaining study. Participants were inadequately controlled before randomisation in all but seven studies. Compared to ICS alone, the addition of LABA to ICS was not associated with a significant reduction in exacerbations requiring oral steroids (seven studies, RR $0.9295 \%$ CI 0.60 to 1.40). Compared to ICS alone, there was a significantly greater improvement in FEV1 with the addition of LABA (nine studies; 0.08 Litres, $95 \%$ CI 0.06 to 0.11 ) but no statistically significant group differences in symptom-free days, hospital admission, quality of life, use of reliever medication, and adverse events. Withdrawals occurred significantly less frequently with the addition of LABA.

(2) A total of seven studies assessed the addition of LABA to ICS therapy compared with an increased dose of ICS randomising 1021 children. The mean age of participants was 8 years with $67 \%$ of males. The baseline mean $\mathrm{FEV}_{1}$ was $80 \%$ of predicted or above in 2 of the 3 studies reporting this characteristic. All trials enrolled participants who were inadequately controlled on a baseline dose equivalent to $400 \mathrm{mcg} / \mathrm{day}$ of beclomethasone or less. There was no group significant difference in the risk of an exacerbation requiring oral steroids with the combination of LABA and ICS compared to a double dose of ICS (two studies, RR $1.595 \%$ CI 0.65 to 3.48). The increased risk of hospital admission with combination therapy was also not statistically significant (RR 2.21 95\% CI 0.74 to 6.64). Compared to double dose ICS, use of LABA was associated with a significantly greater improvement in morning PEF (four studies; MD $7.55 \mathrm{~L} / \mathrm{min}$ 95\% CI: 3.57 to 11.53 ) and evening PEF L/min (three studies, MD 5.5 L/ min; 95\% CI 1.21 to 9.79), but there were insufficient data to aggregate data on $\mathrm{FEV}_{1}$, symptoms, rescue reliever use, and quality of life. There was no statistically significant difference in the overall risk of all cause withdrawals (five studies; RR $0.71 ; 95 \%$ CI 0.42 to 1.20 . There was no group difference in the risk of overall adverse effects detected. Short term growth was significantly greater in children treated with combination therapy compared to double dose ICS (two studies: MD 1.2 $\mathrm{cm} /$ year; $95 \%$ CI 0.72 to 1.7 ).

Authors' conclusions-In children with persistent asthma, the addition of LABA to ICS was not associated with a significant reduction in the rate of exacerbations requiring systemic steroids, but was superior for improving lung function compared to the same dose of ICS. Similarly, compared to a double dose ICS, the combination of LABA and ICS did not significantly increase the risk of exacerbations requiring oral steroids, but was associated with a significantly greater improvement in PEF and growth. The possibility of an increased risk of rescue oral steroids and hospital admission with LABA therapy needs to be further examined. 


\section{Medical Subject Headings (MeSH)}

Adrenal Cortex Hormones [*administration \& dosage; adverse effects]; Adrenergic beta-Agonists [*administration \& dosage; adverse effects]; Albuterol [administration \& dosage; analogs \& derivatives]; Anti-Asthmatic Agents [*administration \& dosage; adverse effects]; Asthma [drug therapy]; Beclomethasone [administration \& dosage; adverse effects]; Drug Therapy, Combination; Ethanolamines [administration \& dosage]

\section{MeSH check words}

Adolescent; Child; Female; Humans; Male

\section{BACKGROUND}

Inhaled corticosteroids (ICS) are the most effective treatment for long-term control of asthma in children (Adams 2005; Adams 2008a; Manning 2008). They are recommended as first line agent in management of childhood asthma in all national and international consensus statements. When ICS are insufficient to achieve control, various options may be considered, such as increasing the dose of inhaled corticosteroids (Adams 2008b), or adding a second drug such as a long-acting beta-2 agonist (LABA) or a leukotriene receptor antagonist (Ducharme 2006).

In adults with unsatisfactory control, international guidelines clearly favour the addition of LABA to low or moderate doses of inhaled steroids over other options such as increasing the dose of steroids or adding other agents. In children, however, recommendations regarding the dose of ICS to which LABA should be added differ markedly across national boundaries. The British Thoracic Society guidelines recommend the combination therapy at a low dose (200-400mcg /day beclomethasone equivalent, BTS 2007). In contrast, Canadian and Australian guidelines recommend the addition of LABA to a higher dose $(800 \mathrm{mcg} / \mathrm{day}$, Canadian Paediatric Asthma Consensus Guidelines; NAC Guidelines 2006). In the American guidelines no clear preference is given between adding LABA to a low dose ICS or increasing the dose ICS in children greater than 5 years of age with moderate asthma; in children with severe asthma, the addition of LABA is preferred option (NIH Publication 2007). Finally, the international GINA guidelines recommend the addition of LABA as an option, irrespective of the baseline dose of ICS $(100-800 \mathrm{mcg} /$ day beclomethasone equivalent, GINA 2007). There is no formal recommendation for their use in the pre-school age group. The wide divergence of recommendations is likely to stem from the lack of solid evidence in children, which may be helped by a systematic review of the topic.

Data from paediatric clinical trials have been included in three previous meta-analyses assessing the effects of LABA. Bisgaard 2003 cautioned against the routine use of LABAs in children, since they did not offer protection against exacerbations and led to an increased risk of hospital admission. Other outcomes such as adverse effects, lung function and symptoms were not examined. More recent work has failed to detect a significant increase in the risk of exacerbations in children, although the direction of effect favoured placebo and the definition of this outcome was not consistent across the studies (OR 1.15 (95\% CI 0.88 
to 1.49; Walters 2007). In both Bisgaard 2003 and Walters 2007, the presence of ICS as background therapy varied between eligible trials. In 2005 we published a Cochrane review combining data from 24 adult and 10 paediatric trials (12 control-intervention comparisons), demonstrating that LABA and ICS led to a significant reduction in risk of exacerbations requiring oral steroids compared with ICS alone (Ni Chroinin 2005). With additional published and unpublished paediatric trials available, we believe that this separate review focused solely on children receiving co-treatment with ICS would shed more light on the role of LABA as an adjunct therapy to ICS in the management of children partially controlled on ICS alone.

\section{OBJECTIVES}

The objective of this review was to assess the safety and efficacy of adding a LABA to ICS in children and adolescents with asthma. We also wished to determine if the benefit of LABA was influenced by baseline severity of airway obstruction, the dose of ICS to which it was added or with which it was compared, the type of LABA used, the number of devices used to deliver combination therapy, and trial duration.

\section{METHODS}

\section{Criteria for considering studies for this review}

Types of studies-Only randomised controlled trials conducted in children, in whom a LABA was added to ICS were eligible.

Types of participants-Children aged two to 18 years with persistent asthma and having received daily ICS therapy for at least 28 days prior to study entry.

Types of interventions-LABA (salmeterol or formoterol) versus placebo administered daily for at least 28 days. The addition of LABA to ICS compared with:

1. the same ICS dose or

2. an increased dose of inhaled corticosteroids.

Studies where maintenance ICS therapy was interrupted for the purposes of run-in were not eligible for the review. Other co-interventions such as xanthines, anticholinergics and other antiasthmatic medications were permitted, provided that the dose remained unchanged throughout the study. Inhaled short-acting $\beta_{2}$-agonists (SABA) and short courses of systemic steroids were allowed as rescue medications.

\section{Types of outcome measures}

Primary outcomes: The primary outcome was the number of asthma exacerbations of moderate intensity, that is, requiring a short course of systemic corticosteroids.

\section{Secondary outcomes}

1. Admissions to hospital

2. Pulmonary function test (morning and evening peak flow; FEV1) 
3. Symptoms

4. Quality of life scores

5. Use of rescue SABA

6. Changes in measures of inflammation such as serum eosinophil cationic protein and sputum eosinophils.

7. Rates of clinical and biochemical adverse effects were examined.

8. Any adverse effect including growth, adrenal suppression, and bone mineral. A suite of related Cochrane reviews in consider serious adverse effects (Cates 2008a; Cates 2009a; Cates 2009b).

\section{Search methods for identification of studies}

As authors of previous related Cochrane reviews (Ni Chroinin 2005; Greenstone 2005) in adults and children, we had reviewed the literature until April 2004. For the present review, we updated the search to May 2008 using the following search strategies:

Electronic searches-An electronic literature search was carried out in the Cochrane Airways Group Specialised Register of asthma trials which is derived from systematic searches of bibliographic databases including the Cochrane Central Register of Controlled Trials (CENTRAL), MEDLINE, EMBASE and CINAHL and handsearching of respiratory journals and meeting abstracts. This Register also contains a variety of studies published in foreign languages. We did not exclude trials on the basis of language. The Register was searched using the following terms:

(((beta* and agonist*) and long-acting or "long-acting") or ((beta* and adrenergic*) and long-acting or "long-acting") or (bronchodilat* and long-acting or "long-acting") or (salmeterol or formoterol or advair or symbicort)) and (((steroid* or glucocorticoid* or corticosteroid*) and inhal*) or (budesonide or beclomethasone or fluticasone or triamcinolone or flunisolide)).

This search was then limited with the text word terms (child* or paediat* or pediat* or adolesc* or infan* or toddler* or bab* or young* or preschool* or "pre school*" or preschool* or newborn* or "new born*" or new-born* or neo-nat* or neonat*)

Searching other resources-Reference lists of all included studies and reviews were checked to identify potentially relevant citations.

We searched manufacturers' and clinical trial websites (Glaxo Smith Kline clinical trials website;

AstraZeneca clinical trials website; Novartis clinical trial results website; Clinical Study Results), to identify other published or unpublished study data. 


\section{Data collection and analysis}

Selection of studies-From the title, abstract or descriptors, the review authors (MNC \& TL) independently reviewed the literature searches. All studies that were not randomised controlled trials or that clearly did not fit the inclusion criteria were excluded. All other citations were reviewed independently in full text by two review authors to assess eligibility.

Data extraction and management-Data for the trials were independently extracted by two review authors (MNC \& TL) in Excel spreadsheets and entered into the Cochrane Collaboration software program Review Manager. Where necessary expansions of graphic reproductions and estimations from other data presented in the paper were performed. Primary authors or sponsors were requested to confirm the methodology and data extraction and were asked to provide additional information as needed.

We recorded as a 'User defined order':

1. the mean daily dose of inhaled corticosteroids in trials where both the intervention and control groups used the same dose of inhaled steroid and

2. the dose difference between the two groups in studies comparing the addition of LABA to ICS with an increased dose of inhaled corticosteroid. Both values were reported in chlorofluorocarbon (CFC)-propelled beclomethasone-equivalents, where $1 \mu \mathrm{g}$ of beclomethasone dipropionate equates to $1 \mu \mathrm{g}$ of budesonide and 0.5 $\mu \mathrm{g}$ of fluticasone propionate (NIH Publication 2007). All doses of inhaled medications were reported as ex-valve, rather than ex-inhaler, values.

Assessment of risk of bias in included studies-We assessed the risk of bias according to five domains:

1. Allocation generation \& concealment

2. Blinding

3. Handling of missing data

4. Selective reporting bias

5. Information on the proportion of the screened population that meet the eligibility criteria.

For each domain we judged the risk of bias as being low ('Yes'), unclear ('Unclear'), and high ('No'), in line with recommendations from the Cochrane Handbook.

Unit of analysis issues-We excluded crossover studies from contributing data to dichotomous measurements of exacerbations since we used analyses that assume measurements are taken from independent samples.

Dealing with missing data-We contacted study investigators (or study sponsors where trials had pharmaceutical company sponsorship) directly to obtain confirmation of the methodology and to obtain data missing from the original trial report. We primarily sought data on the outcomes relating to exacerbations (oral steroid use and admission to hospital). 
Assessment of heterogeneity-Homogeneity of effect sizes between pooled studies was examined with the $\mathrm{I}^{2}$ statistic using $25 \%$ or more as a cut-off for exploring possible causes of heterogeneity (Higgins 2003). In the absence of heterogeneity, the fixed-effect model was used, otherwise the Dersimonian and Laird random-effects model (DerSimonian 1986) was applied to the summary estimates. Results of the fixed effects model have been reported unless otherwise stated in the text.

Assessment of reporting biases-Funnel plots were used to check for the indications of possible publication bias.

Data synthesis-Treatment effects for dichotomous variables were calculated as a pooled relative risk (RR) with 95\% confidence intervals (CI). For continuous outcomes, such as pulmonary function tests, pooled statistics were calculated as weighted mean differences (MD) or standardized mean differences (SMD) if results were on different scales, and reported with $95 \%$ confidence intervals. When standard deviations were not presented but could be estimated from an effect estimate and either confidence intervals, standard error, or $\mathrm{P}$-value, we combined the mean differences with the generic inverse variance function in Review Manager (GIV).

Equivalence was assumed if the relative risk estimate and its confidence interval were between 0.9 and 1.1. Numbers needed to treat (NNT) were derived from the pooled Risk Ratios using Visual Rx.

The analysis examined two main comparisons namely the combination of LABA and ICS to:

1. A similar dose of ICS with placebo, representing step 2 of the BTS guidelines.

2. An increased dose of ICS with placebo, representing step 3 of the BTS guidelines.

When a trial tested more than two arms, additional control-intervention group comparisons were considered for this review. If the same group was used twice as a comparator in a three-arm study, the number of participants in the group used twice was halved to avoid over-representation. For event rates, the denominator was also halved in the control group. (Zimmerman 2004b; Zimmerman 2004a; Pohunek 2006a; Pohunek 2006b; SD 039 0725a; SD 039 0725b; Morice 2008a; Morice 2008b).

Subgroup analysis and investigation of heterogeneity-Subgroup analyses were planned to explore possible reasons for heterogeneity and, in the absence of heterogeneity, to identify potential effect modifiers where the magnitude of benefit may vary according to baseline characteristics. The following a priori defined subgroups were examined:

1. Magnitude of airway obstruction at baseline as determined by the mean percent predicted forced expiratory volume in one second $\left(\mathrm{FEV}_{1}\right)$ : classified as mild $(80 \%$ of predicted or more), moderate (61 to $79 \%$ of predicted) or severe (60\% of predicted or less) (GINA 2007).

2. Mean dose (ex-valve) of ICS in comparison 1 when comparing LABA + ICS versus placebo + ICS and the dose difference in comparison 2 when comparing 
LABA + ICS versus increased dose of ICS, both reported in CFC-propelled beclomethasone-equivalent doses ( $\mu \mathrm{g} / \mathrm{day})$ and portrayed as the user-defined number

3. Usual versus higher than usual dose (reported as ex-valve in $\mu \mathrm{g}$ ) of the LABA (salmeterol or formoterol).

4. Type of LABA (salmeterol versus formoterol).

5. Use of one or two devices to deliver the combination of ICS and LABA.

6. Trial duration comparing trials $\leq 16$ weeks to those $\geq 24$ weeks.

Sensitivity analysis-Sensitivity analyses were performed to assess whether the results of our primary outcome was sensitive to blinding and completeness of follow-up, publication status, and funding.

\section{RESULTS}

\section{Description of studies}

See: Characteristics of included studies; Characteristics of excluded studies.

\section{Results of the search}

See Figure 1 for an overview of the literature search results, their assessment and inclusion of studies in the review. Updated electronic and additional handsearches from February 2004 to May 2008 retrieved 337 additional citations. We included 15 new trials, giving a total of 25 eligible trials.

Six of the trials generated an additional control-intervention comparison. Three trials (representing six control-intervention comparisons) assessed two forms of additive therapy (ICS and LABA via one inhaler or via two inhaler devices against one dose of ICS): Morice 2008a; Morice 2008b; Pohunek 2006a; Pohunek 2006b; SD 039 0725a; SD 039 0725b. Three trials (representing six control-intervention comparisons) assessed one form of ICS and LABA against two doses of ICS (SAM40012a; SAM40012b; Verberne 1998a; Verberne 1998b; Zimmerman 2004a; Zimmerman 2004b). The review therefore lists 31 controlintervention comparisons.

\section{Included studies}

The trials randomized 5,572 children. Sixteen were available as full text publications and nine were unpublished reports of trials accessed from pharmaceutical company trials registries.

The majority of studies were funded by producers of both LABA and ICS inhalers: ten were supported by GSK; nine by Astra Zeneca; one by Allen \& Hanburys, a subsidiary of GSK in the United Kingdom (Russell 1995). Only two were independently supported by a charitable organization (Langton Hewer 1995; Stelmach 2007), and three failed to identify the source of funding (Heuck 2000; Teper 2005; Zimmerman 2004a; Zimmerman 2004b). 
The studies were classified in to one of two comparisons according to the research question addressed. In accordance to with therapeutic management recommended by GINA 2007 and BTS 2007, patients on ICS alone were considered as receiving step 2 therapy. The comparison of the addition of LABA versus placebo to the same ICS dose, were referred to Step 3 versus Step 2 comparison (24 control-intervention comparisons). Comparisons testing the combination of LABA and ICS to a double dose of the ICS used before randomisation are hereafter referred to a Step 3 versus Step 3 comparison (seven controlintervention comparisons). Bisgaard 2006 examined the addition of LABA to a lower dose of ICS (BDP 100mcg) than has been advocated by international guidelines as Step 3, and was included in the Step 3/Step 3 comparison.

We describe hereafter the characteristics of the studies which contribute outcome data to one or more comparisons in the review. Full study description of each eligible study see Characteristics of included studies.

\section{Long-acting betaß2 agonist + inhaled corticosteroid versus same dose of inhaled corticosteroid as used prior to randomisation (Step 3/Step 2)-Twenty-}

four control-intervention comparisons randomising 4,625 children assessed the addition of LABA versus placebo with the same dose of ICS in the control group (Akpinarli 1999; Langton Hewer 1995; Meijer 1995; Morice 2008a; Morice 2008b; Pohunek 2006a; Pohunek 2006b; Russell 1995; SAM40012a; Malone 2005; SD 039 0714, SD 039 0718; SD 039 0719; SD 039 0725a; SD 039 0725b; SFA100314; SFA100316; Simons 1997; Stelmach 2007; Tal 2002; Teper 2005; Verberne 1998a; Zimmerman 2004b and Zimmerman 2004a).

Participants-The mean age of participants was 10 years and males accounted for $64 \%$ of the study populations. Mean $\mathrm{FEV}_{1} \%$ predicted at baseline was $80 \%$ predicted or above) in 10 control-intervention comparisons (Langton Hewer 1995; Malone 2005; Meijer 1995; Pohunek 2006a; Pohunek 2006b; SD 039 0718; SD 039 0719; Simons 1997; Teper 2005; Verberne 1998a); $\mathrm{FEV}_{1} 61 \%$ to $79 \%$ of predicted) in eight control-intervention comparisons Akpinarli 1999; Russell 1995, SD 039 0714, SD 039 0725a; SD 039 0725b; Tal 2002; Zimmerman 2004b; Zimmerman 2004a) and unreported in the remaining studies. Participants were inadequately controlled before randomisation in all but five studies where they were described as well controlled (Meijer 1995; Pohunek 2006a; Pohunek 2006b; Simons 1997) or where control was unreported (SD 039 0725a; SD 039 0725b; SFA100314; SFA100316; Teper 2005).

Interventions-Salmeterol was assessed in 10, and formoterol in 14, control-intervention comparisons. All but one comparison tested the usual recommended dose of the long-acting B2-agonist (that is, salmeterol $50 \mu \mathrm{g}$ twice daily, formoterol 6 or $12 \mu \mathrm{g}$ twice daily); in Langton Hewer 1995, salmeterol 100 bid was used. The dose of inhaled steroid (BDP equivalent) was 200mcg/d in four studies (SD 039 0718; SD 039 0725a; SD 039 0725b; Stelmach 2007); $400 \mathrm{mcg} / \mathrm{day}$ in 11 (46\%) control-intervention comparisons (Morice 2008a; Morice 2008b; Pohunek 2006a; Pohunek 2006b; SAM40012a; SFA100314; SFA100316; Malone 2005; SD 039 0714; Tal 2002; Verberne 1998a) 500mcg/day was used in two studies (Meijer 1995; Teper 2005); 800mcg in SD 0390719 and an unspecified/varied dose 
in six studies (Akpinarli 1999; Langton Hewer 1995; Russell 1995; Simons 1997;

Zimmerman 2004a; Zimmerman 2004b).

Twelve (50\%) control-intervention comparisons assessed the combination of LABA and ICS in a single device, the remainder assessed the efficacy and safety of a LABA administered separately to ICS.

Trial duration varied from eight weeks or less in six studies (Akpinarli 1999; Simons 1997; Langton Hewer 1995; SFA100314; SFA100316; Stelmach 2007), 12-16 weeks in 14 control-intervention comparisons (Meijer 1995; Morice 2008a; Morice 2008b; Pohunek 2006a; Pohunek 2006b; Russell 1995; Malone 2005; SD 039 0714; SD 039 0718; SD 039 0725a; SD 039 0725b; Tal 2002; Zimmerman 2004b; Zimmerman 2004a), 24-26 weeks in two studies (SAM40012a; SD 039 0719) and one year in two studies (Teper 2005; Verberne 1998a).

Although co-intervention with other prophylactic medications was permitted, trial protocols stipulated that their doses should remain unchanged throughout. The proportion of patients on additional therapy was not consistently reported. Permitted drugs included systemic steroids, anticholinergics, and xanthines (Langton Hewer 1995), immunotherapy (Zimmerman 2004b; Zimmerman 2004a), unspecified agents (Russell 1995). Other preventative medication were not permitted in the other studies except for Teper 2005, where this was unspecified. Rescue medications such as inhaled short-acting 32 -agonists and systemic steroids were permitted in all the studies.

Outcomes-The primary outcome of this review is the proportion of participants who experienced exacerbations requiring systemic steroids. This was available for eight studies. Where data were not reported, or were only described in a format we could not use directly, we requested study sponsors to provide further information. Our requests for data on exacerbations requiring rescue oral steroids for Morice 2008a; Morice 2008b; Pohunek 2006a; Pohunek 2006b; SD 039 0714; SD 039 0718; SD 039 0719; SD 039 0725a; SD 039 0725b; Stelmach 2007 have not been successful.

Hospital admission data were available for six studies. A measurement of lung function was reported in all studies. Symptoms were reported in all studies except for (SD 039 0714; Simons 1997). Data on rescue 32 -agonist were available for all but one study (Simons 1997). Withdrawals were reported in all but three studies (Akpinarli 1999; Meijer 1995; Teper 2005). Adverse events were reported in all studies.

\section{Long-acting betaß2 agonist + Inhaled corticosteroid versus increased dose of inhaled corticosteroid (Step 3/Step3)—A total of seven studies assessed the addition of LABA versus placebo to ICS therapy with increased dose of ICS in the control group (Bisgaard 2006; Heuck 2000; Ortega-Cisneros 1998; SAM40012b; SAM40100; SAM104926 and Verberne 1998b). One study did not contribute data (Ortega-Cisneros 1998). The studies randomised 1048 children.}


Participants-The mean age of participants was 10 years with $77 \%$ of males. Baseline airway obstruction was reported in three of the six studies (SAM104926: 102\%; Verberne 1998b: 87\%; Bisgaard 2006: 75\%). Heuck 2000 recruited children with mild asthma; however, the remaining trials recruited participants whose asthma was inadequately controlled asthma at the time of enrolment on inhaled steroids.

Interventions-Salmeterol and formoterol were studied in four and three studies respectively. All of the comparisons tested the usual recommended dose of the long-acting B2-agonist (i.e. salmeterol $50 \mu \mathrm{g}$ twice daily, formoterol 6 or $12 \mu \mathrm{g}$ twice daily). Intervention groups in four studies received BDP equivalent doses of $400 \mathrm{mcg} /$ day (SAM40012b; SAM104926; SAM40100; Verberne 1998b). BDP at 100mcg/d was used in Bisgaard 2006 and $200 \mathrm{mcg} / \mathrm{day}$ was used in Heuck 2000. The respective control groups received double the dose of inhaled steroid administered as the intervention group dose. Four studies assessed LABA and ICS as a single inhaler administration (Bisgaard 2006; SAM40012b; SAM40100; SAM104926).

Study duration was 6 weeks in SAM40100, 12-16 weeks in three studies (Bisgaard 2006; Heuck 2000; SAM104926), 24 weeks in one comparison (SAM40012b), and one year for the one (Verberne 1998b).

All studies recruited children who were taking an inhaled steroid at baseline. Rescue medications such as inhaled short-acting B2-agonists and systemic steroids were permitted in all the trials.

Outcomes-Data on oral steroid exacerbations were available from three studies (Heuck 2000; SAM104926; Verberne 1998b). Since Heuck 2000 was a crossover study however, we were not able to combine dichotomous data from this with the other trials. Where data were not reported, or were described for an undefined exacerbation or composite of types of exacerbations, we requested study sponsors to provide further information. Our requests for data on exacerbations requiring rescue oral steroids from study sponsors for Bisgaard 2006; SAM40012b; SAM40100 have not been successful.

Hospital admission data were available for two studies. Lung function outcomes were available for all studies. Data on symptoms, rescue $\beta_{2}$-agonist use, adverse events and withdrawals were reported in all studies. Two studies provided data on growth (Bisgaard 2006, Verberne 1998b).

\section{Excluded studies}

Details of 63 studies which did not meet the eligibility criteria of our review are given in Characteristics of excluded studies (this number is drawn from all years searches to May 2008 across this review, Greenstone 2005 and Ni Chroinin 2005).

\section{Risk of bias in included studies}

An overview of judgements on domains relating to the risk of bias is provided in Figure 2. We summarise our findings below. 
Verification of study details with study authors was obtained for six control-intervention comparisons (Pohunek 2006a; Pohunek 2006b; Russell 1995; Simons 1997; Verberne 1998a; Verberne 1998b). Information on the design of GSK-sponsored studies was provided in correspondence (Appendix 1).

Allocation-In 19 studies randomisation procedures were adequate in reducing the risk of group imbalances at baseline; in the remaining 12 studies this was unclear.

Blinding - Blinding of intervention was maintained with double-dummy designs, or the use of identical inhaler devices in 29 studies. Two studies had an open-label design; one study primarily designed to assess safety outcomes (SD 039 0719), the other study did not contribute any numerical outcome data (Ortega-Cisneros 1998).

Incomplete outcome data-There was a lack of information available on the definition of intention to treat principles across the studies. Our judgments of this aspect of the studies reflect uncertainty over the reliability of the stated methods.

Selective reporting-The availability of our pre-specified primary outcome, patients with exacerbations requiring rescue systemic steroids, from the reports of trials was limited. This is partly explained by different definitions of exacerbation used by investigators across the studies. Despite extensive efforts to obtain data for our primary outcome we have only a limited amount of data available for analysis. We remain uncertain as to whether data for this end-point were collected in 10 studies (Langton Hewer 1995; Meijer 1995; OrtegaCisneros 1998; Pohunek 2006a; Pohunek 2006b; SAM104926; SAM40100; Stelmach 2007;

Tal 2002; Teper 2005).

\section{Effects of interventions}

\section{Long-acting betaß2 agonist + inhaled corticosteroid versus same dose of inhaled corticosteroid (Step 3 versus Step 2)}

Primary outcome: There was no statistically significant difference in the risk of exacerbations requiring systemic corticosteroids between treatments (seven studies; RR 0.92 $95 \%$ CI 0.60 to $1.40, \mathrm{~N}=1,088$; Figure 3 ).

Subgroup analysis: Since the number of studies was low, together with inconsistent reporting of characteristics, the impact of the baseline severity of airway obstruction, baseline dose of ICS, type of LABA, and trial duration on the magnitude of effect could not be examined.

Sensitivity analysis: With all the studies being double-dummy or used identical inhaler devices, sensitivity analysis on the quality of blinding was not done. The removal of two studies where follow-up of randomized patients was at a high risk of bias gave a similar effect size to the primary analysis (RR $0.9995 \%$ CI 0.53 to 1.85 ). Removing three studies where data were available on request, and not from full text sources, gave a pooled result of RR 0.9 ( 0.57 to 1.42$)$. All studies were funded by manufacturers of the products. 


\section{Secondary outcomes}

Hospital admission, serious adverse events \& withdrawal: There was no statistically significant difference in exacerbations requiring admission to hospital (five studies, RR 1.65 95\% CI 0.83 to 3.25, Figure 4), serious adverse events (seventeen studies, RR $1.1695 \%$ CI 0.73, 1.85; Analysis 1.3), withdrawals due to poor asthma control (six studies, RR 0.69; $95 \%$ CI: 0.34 to 1.4; Analysis 1.5). Withdrawal due to any reason was lower with LABA compared to placebo (21 studies, RR 0.79 95\% CI 0.67 to 0.93; Analysis 1.4).

Lung function: The addition of LABA to ICS provided significantly greater improvement from baseline in lung function compared to using placebo and ICS. This was true, irrespective of whether the group differences ere reported for $\mathrm{FEV}_{1}$ as change in litres (nine studies; 0.08 Litres, $95 \%$ CI 0.06 to 0.11, random-effects model; Analysis 1.8), change in \% predicted (2.35\% 95\% CI 0.07 to 4.64; Analysis 1.9) in \% predicted at endpoint (3.79\%; 95\% CI 1.99 to 5.60; Analysis 1.10), as change in morning peak expiratory flow (14 studies; MD $10.38 \mathrm{~L} / \mathrm{min}, 95 \%$ CI 8.23, 12.52, random-effects model; Analysis 1.14) or evening peak expiratory flow, (11 studies, MD 9.37 L/min, 95\% CI 6.96 to 11.79; Analysis 1.17). The severity of airway obstruction at baseline had no apparent effect of the magnitude of improvement in lung function when assessed using morning PEF ( $\mathrm{P}=0.647)$. Duration of intervention did not affect the magnitude of improvement in FEV1 over time (Analysis 1.11). The favourable effect of LABA was sustained from 6 to 24 weeks, which did not support the presence of tachyphylaxis. The studies contributing data to lung function endpoints recruited children with mild to moderate airway obstruction, with a range of lung function at baseline and examined the effects of both salmeterol and formoterol, given in conjunction with a range of doses of ICS.

Symptoms, rescue beta-agonist use \& quality of life: The addition of LABA did not result in significant group differences for the following outcomes:

Change in mean symptom scores (four studies, SMD -0.04 ; $95 \%$ CI -0.16 to 0.08 ; Analysis 1.22).

$\%$ symptom-free days as end of treatment values (outcome 01.24; two studies, WMD 0.07; $95 \% \mathrm{CI}-0.14$ to 0.27 ; Analysis 1.27). Change in symptom-free days (three studies WMD $1.30 \%$; $95 \%$ CI -3.12 to 5.71 ; Analysis 1.26 ).

Change in daytime use of rescue short-acting B2-agonists (five studies, WMD -0.07 puffs/ day, $95 \%$ CI -0.14 to 0.01 ; Analysis 1.20 ).

$\%$ days without relief medication use (three studies, WMD $-0.56 \%$; $95 \%$ CI -2.84 to 1.73 ; Analysis 1.21).

Asthma quality of life questionnaire scores (PAQLQ; ten studies, WMD 0.03; 95\% CI -0.04 to 0.11; Analysis 1.29).

There was an insufficient number of studies to combine data on other outcomes relating to the change in number of night time rescue inhalations (Analysis 1.23), change in night time 
awakenings (Analysis 1.24), \% nights with awakening (Analysis 1.25), \% symptom-free nights (Analysis 1.28), and non-specific cardiovascular events (Analysis 1.35).

Tolerability: There was no statistically significant difference in the risk of overall adverse effects (15 studies, RR 1.04; 95\% 0.98 to 1.10; Analysis 1.30), reaching our a priori defined criteria for equivalence. Specifically, there was no significant group difference in the risk of oral candidiasis (two studies; RR 3.78, 95\% CI: 0.63, 22.75; Analysis 1.32); tremor (two studies; RR 3.07; 95\% CI 0.38, 25.05; Analysis 1.33), palpitations (two studies; RR 0.4 95\% CI: 0.05 to 3.25; Analysis 1.34), and headache (14 studies, RR $1.1095 \%$ CI 0.90 to 1.33 ; Analysis 1.31). The large confidence interval for specific adverse events, due to the small number of reporting trials, rules out total reassurance.

Although the effect on growth could not be aggregated because only one study documented this outcome (Verberne 1998a), there was no statistically significant group difference in the growth velocity over 52 weeks in prepubertal children (mean age 10-11 years) comparing BDP $400 \mathrm{mcg}+$ Salmeterol to BDP $400 \mathrm{mcg}$ alone (5.1 cm versus $4.5 \mathrm{~cm}$ respectively). No studies reported deaths. The risk of withdrawals due to adverse effects was not significantly different between groups (18 studies, RR 0.78 95\% CI 0.52 to 1.19; Analysis 1.7).

\section{Long-acting $B 2$ agonist and inhaled corticosteroid versus increased dose of inhaled corticosteroids (Step 3 versus Step3)-Six studies contributed data to} outcomes under this comparison (Bisgaard 2006; Heuck 2000; SAM40012b; SAM104926; SAM40100; Verberne 1998b).

Primary outcome: Despite correspondence with study sponsors to obtain data on exacerbations requiring rescue oral steroids, data was obtained from only two studies SAM104926; Verberne 1998b. There was no significant group difference in the risk of patients having an exacerbation requiring oral steroids between LABA+ICS and higher dose ICS (RR $1.595 \%$ CI 0.65 to 3.48, two studies, $\mathrm{N}=441$ Figure 5).

Secondary outcomes: There was no significant group difference in the risk of exacerbations requiring hospitalisation (four studies; RR $2.2195 \%$ CI 0.74 to 6.64, Figure 6), or the risk of serious adverse events (RR $1.4595 \%$ CI 0.63 to 3.33, Analysis 2.3). There was no statistically significant difference in the overall risk of all cause withdrawals (five studies; RR $0.71 ; 95 \%$ CI 0.42 to 1.20 , Analysis 2.4 .

The addition of LABA to ICS was led to greater improvement from baseline in the change in morning PEF (four studies; MD 7.55 L/min 95\% CI: 3.57 to 11.53, Analysis 2.10), evening PEF L/min (three studies, MD 5.5 L/min; 95\% CI 1.21 to 9.79, Analysis 2.11). Change from baseline in FEV1 was not statistically significant between treatment options (2 trials, MD $0.01 \mathrm{~L}, 95 \% \mathrm{CI}-0.03,0.05$ ) (Analysis 2.7). There was insufficient data to pool other lung function data.

There were insufficient studies to aggregate other data related to symptoms including number of daytime rescue inhalations, number of nighttime awakenings, daytime symptoms, and nighttime symptoms. 
There was insufficient number of trials to aggregate data on inflammatory mediators.

There was no statistically significant difference in the risk of overall adverse effects: four studies; RR $1.0595 \%$ CI 0.90 to 1.23 , Analysis 2.15 , failing to meet our a priori criteria for equivalence. The relative risk of headache (three studies; RR 1.37 95\% CI 0.98 to 1.90; Analysis 1.31), or withdrawal due to adverse effects (two studies; RR 1.44 95\% CI 0.25 to 8.42, Analysis 2.5) did not reach statistically significant differences. Growth over one year was measured in two studies (Verberne 1998b; Bisgaard 2006); the findings favoured LABA-treated children by an average of $(1.2 \mathrm{~cm} /$ year $95 \%$ CI 0.72 to 1.7 , Analysis 2.17$)$.

\section{DISCUSSION}

In school-aged children insufficiently controlled on inhaled corticosteroids (ICS), there is no statistically significant benefit of adding long-acting beta2-agonist (LABA) to ICS compared to using the same or an increased dose of ICS for preventing exacerbations requiring systemic steroids. Lung function endpoints consistently favoured the addition of LABA to ICS therapy, whether compared to the same dose or increased dose ICS. Irrespective of the intervention compared to the combination of LABA and ICS, it was not possible to identify the characteristics of patients (age, severity of airway obstruction, duration of disease) or the characteristics of the intervention (ICS dose, duration of treatment) associated with greatest benefit. The addition of LABA to ICS did not result in significantly greater improvement in symptoms or reduction in the use of rescue 32 -agonist (SABA) compared with the same dose of ICS, and there were insufficient data to assess these outcomes comparing LABA with an increased ICS dose. With one notable exception, there was no statistically significant group differences in reported adverse events, but the wide confidence interval for most outcomes preclude reassurance. It was noteworthy that the combination of LABA and ICS lead to a greater gain in linear growth than an increased dose of ICS by 300 to $400 \mathrm{mcg} / \mathrm{day}$. Moreover, while not statistically significant, there was also a trend towards a more than two-fold increase in the risk of exacerbations requiring systemic steroids and hospital admissions respectively, in children treated with combination therapy, compared with a double dose of ICS; the possibility of increased risks of such exacerbations on combination therapy will need to be tested in future trials.

The lack of an effect of combination therapy on exacerbations requiring systemic steroids when used as step 3 compared to same dose of ICS (step 2) is concordant with findings of Bisgaard 2003 and Walters 2007. We must interpret these findings in view of the fact that in our review most data were derived from school-aged children with normal or near normal lung function; they were predominantly treated with ICS and salmeterol delivered in separate devices, and the dose of maintenance ICS varied across the studies. One may anticipate different results in more severe patients, treated for longer, although this is not suggested by the subgroup analysis of children with moderate (RR $0.8995 \%$ CI 0.48 to 1.64) versus mild (RR $0.8795 \%$ CI 0.47 to 1.64 ) airway obstruction.

There are some aspects of our findings which contrast with estimates derived from our prior systematic review of adult trials. Indeed, when compared with a similar dose of ICS, the addition of LABA to ICS reduces by $20 \%$ the risk of adults with exacerbations requiring 
systemic steroids (RR 0.80; 95\% CI 0.73 to 0.89 ; Ni Chroinin 2005). This was accompanied by a notably greater improvement in lung function $(170 \mathrm{~mL}$ in FEV1) and symptom-free days $(+17 \%)$ and a modest reduction in the use of rescue SABA ( -0.7 puffs/days). In this paediatric review, other than an improvement in lung function, the addition of LABA to ICS did not result in improvements in clinical outcomes. One might argue that considering the smaller lung volumes in children, the observed $80 \mathrm{~mL}$ greater improvement in FEV1 associated with the addition of LABA to ICS in children may be clinical importance, similar perhaps to that observed in adults. Yet, the improvement in lung function appears to contradict the lack of protection for exacerbations. This disparity may be due to a more rapid effect of LABA on lung function, more easily detectable in studies of short duration, whereas a longer period of follow-up may be required to detect an effect on exacerbations, particularly in children with normal or near normal lung function. In view of the requirement for significant reversibility with SABA for study eligibility, the observed improvement in FEV1 may also be both expected and specific to these patients, and may not be generalisable to other patients. Hence the importance of other outcomes to judge the benefit of LABA, which unfortunately were not observed in the studies. While the absence of a significant group difference in other outcomes may be due to patient characteristics (age, severity of airway obstruction, duration of asthma) or study design (ICS dose, duration of treatment, reported outcomes and power), the aggregation of the best available evidence provides little data to support the addition of LABA to ICS as a step 3 approach, let alone enables us to identify whom and at which dose this approach may be most effective.

With regard to the second comparison in our review (LABA and ICS versus a higher dose of ICS), our findings differ from those of an ongoing update of a Cochrane review of studies in adults (personal communication Ducharme et al), which demonstrates a significant reduction in the risk of patients with exacerbations requiring rescue systemic steroids.

Disappointingly, despite the identification of six studies, only two provided data for the primary outcome under this comparison. A modest improvement $(<7 \mathrm{~L} / \mathrm{min})$ in morning and evening PEF, but not in FEV1, was associated with the use of LABA compared to a higher ICS dose. Insufficient reporting prevented the aggregation of other outcomes. However, the trends toward an increased risk of exacerbations requiring systemic steroids and admission and serious adverse effects with combination therapy is of concern. Is it possible that ongoing inflammation associated with the use of a lower dose of ICS or tachyphylaxis associated with the prolonged use of LABA may be associated with more severe exacerbations with combination therapy? These concerns call for urgent trials to clarify these observations. Meanwhile, the available data are also insufficient to recommend the preference of any given step 3 strategy over any other. One must weigh the greater linear growth and modest improvement in PEF against the possible but unproven increased risk of increased severity of exacerbations associated with combination therapy.

There was no group difference in adverse effects or withdrawals due to adverse effects when the combination of LABA and ICS was compared to either step 2 or step 3 strategy. Of note, side effects were scarcely reported in the short-term trials and there was a paucity of longterm studies. Moreover, while an increased dose of inhaled corticosteroids calls for the assessment of growth, adrenal function and bone mineralization in children, no trial reported data that could be aggregated on adrenal function and bone mineralization. Growth was 
examined in only two studies examining the addition of LABA to $400 \mathrm{mcg}$ versus $800 \mathrm{mcg}$ of BDP (Verberne 1998b) and to $100 \mathrm{mcg}$ versus $400 \mathrm{mcg}$ of Budesonide (Bisgaard 2006), a differential of 300 to $400 \mathrm{mcg}$ of BDP-equivalent. The observed reduction in growth averaging $1.2 \mathrm{~cm} /$ year is in line with the documented decrease in linear growth associated with $400 \mathrm{mcg} /$ day of BDP (Sharek 1999); the data may also indicate a dose-response relationship with inhaled steroids. Any apparent benefit of doubling the dose ICS should be weighed against the possible impact on growth compared to other therapeutic regimes, and deserves careful evaluation.

Although we have managed to identify a number of unpublished studies, we have had only limited success in obtaining usable data for our primary outcome. We successfully obtained data for exacerbations requiring rescue systemic steroids and for hospital admissions for a small number of trials from a recent meta-analysis of GSK sponsored trials (Bateman 2008). The availability of data in study reports available as downloads from pharmaceutical company trial results registries was poor. Moreover, the limited response from study authors or sponsors to our requests for providing data contributed to the lack of precision of, and possible bias in, our main outcome. At the time of writing, we are still awaiting the response to requests for data on exacerbations requiring rescue oral steroids from study sponsors for: Bisgaard 2006; SAM104926; SAM40012a; SAM40012b; SAM40100; SD 039 0714; SD 039 0718; SD 039 0719; SD 039 0725a; SD 039 0725b; Zimmerman 2004a; Zimmerman 2004b. These studies represent $52 \%$ of the children randomised.

\section{AUTHORS' CONCLUSIONS}

\section{Implications for practice}

There is insufficient evidence at present to firmly support the use of LABA as adjunct therapy to ICS as a step 3 strategy to reduce the risk of asthma exacerbations requiring steroids, as compared to using the same dose of ICS or an increase in the dose of ICS. The wide confidence intervals do not rule out a superior effect of either treatment; however the trend for an increased risk of exacerbations requiring systemic steroids, hospital admission, and serious adverse health event associated with combination therapy compared with an increased dose of ICS is of some concern, and indicates that trials are urgently required to address this issue. There is clear evidence that stepping up therapy with the addition of LABA improves lung function beyond that observed with remaining on ICS as step 2 strategy, with no apparent effect on symptoms and use of rescue B2-agonists. Significant improvements with LABA in morning PEF over increased dose of ICS have not been associated with improvements in other outcomes and more work is a priority in this area. The apparent reduction in growth associated with use of 400 to $800 \mathrm{mcg} / \mathrm{day}$ of BDPequivalent raises concern if high dose BDP or BUD are considered.

\section{Implications for research}

Future trials should aim for the following characteristics: 
Population

There urgently needs to be a large study in children with more severe asthma than those recruited to the trials in this review. Stratifiication according to age, degree of airway obstruction (i.e. baseline FEV1), and the inclusion of younger, pre-school aged children should feature in the design of such trials. It should also include those with a clinical diagnosis of asthma who do not necessarily have a positive bronchodilator response which would then be more generalisable to the general paediatric asthma population.

\section{Interventions}

Future interventions should examine combination therapy delivered using a single inhaler (combining LABA and ICS) to avoid use of LABA as monotherapy. Interventions may include head-to-head comparisons of salmeterol versus formoterol, combined with low or moderate dose inhaled steroids, as once versus twice daily regimen. The control intervention should include an increased dose of inhaled corticosteroids (step 3) and a similar dose ICS (step 2).

\section{Design}

1. Double blinding, adequate randomisation and complete reporting of withdrawals and dropouts with an explicit definition of the intention-to-treat population analysed.

2. Intervention period of 24 weeks or more to properly assess the impact on exacerbations requiring systemic corticosteroids, and those resulting in hospital admission as well as adverse health events (growth, adrenal function, bone mineralization, serious adverse health events).

3. Clear reporting of the per cent (and reasons) of non-eligibility of approached patients and of those enrolled in the run-in period are required as inadequate reporting of the selected population results in difficulty in identifying to whom the results can be generalized.

4. Complete reporting of continuous (denominators, mean change and mean standard deviation of change) and dichotomous (denominators and rate) data in the units used in this systematic review would allow aggregation of data.

Outcomes of particular importance to assess include:

1. Exacerbations requiring systemic corticosteroid;

2. Asthma-related hospital admission;

3. Compliance to either intervention both pre (for ICS) and post -randomisation (for both ICS and combination therapy). The impact of compliance to combination therapy versus placebo and ICS on the magnitude of the effect size should be examined;

4. Cost effectiveness of use of combination inhalers as compared to inhaled corticosteroids alone; 
5. Long-term side effects of long-acting $ß 2$-agonists on serious adverse effects, and admission and of ICS (growth, adrenal function, bone mineralization);

6. Functional measures including quality of life.

\title{
Acknowledgments
}

\begin{abstract}
We thank the Cross Canada Respiratory Rounds Review Group for their valuable comments. We are indebted to the Cochrane Airways Review Group, namely Stephen Milan, Elizabeth Arnold, Veronica Stewart, Karen Blackhall and Bettina Reuben for the literature search and ongoing support as well as to Peter Gibson and Christopher Cates for their constructive comments. We are indebted to the trialists who provided us with data and information regarding their studies, namely A Tal, E Simons, G Russell, F Meijer, Petr Pohunek, Christine Sorkness and AAPH Verberne. We thank Richard Follows, Tracey Armstrong and Maggie Hemedah from GlaxoSmithKline, Robyn von Maltzahn, Nils Grundstrom and Roger Metcalf from AstraZeneca who cooperated with our requests for information.
\end{abstract}

\section{SOURCES OF SUPPORT}

Internal sources

- $\quad$ St George's University of London, UK.

External sources

- $\quad$ No sources of support supplied

\section{CHARACTERISTICS OF STUDIES}

\section{Characteristics of included studies [ordered by study ID]}

Akpinarli 1999

\begin{tabular}{ll}
\hline Methods & Parallel group, multicentre study. \\
\hline Participants & Symptomatic asthmatic children \\
\% ELIGIBLE OF SCREENED POPULATION: Not reported \\
\% RUN-IN PARTICIPANTS RANDOMISED: Not reported \\
RANDOMISED: 32 (ICS + F12 (BID): 16; ICS: 16) \\
WITHDRAWALS: Not described \\
AGE mean (range) or mean (SD): 6 to 14 years \\
GENDER:(\% male): 47\% \\
SEVERITY: not reported \\
BASELINE \% PRED. FEV1: Not described \\
BASELINE DOSE OF ICS: 400-800mcg \\
ASTHMA DURATION: not described \\
ATOPY (\%): 68 \\
ELIGIBILITY CRITERIA: Met ATS criteria for asthma; >= 15\%increase in FEV1 \\
within the previous year \\
EXCLUSION CRITERIA: Asthma exacerbation or respiratory infection in < \\
month \\
ELIGIBILITY CRITERIA DURING RUN IN: Only patients requiring salbutamol \\
more than once a week were randomised \\
LABA + ICS vs SAME dose of ICS \\
OUTCOMES reported at 6 weeks \\
RUN IN PERIOD: 2 weeks with ICS $400-800$ mcg/day to document symptoms \\
and beta2-use \\
DOSE OPTIMISATION PERIOD: NONE \\
INTERVENTION PERIOD: 6 weeks \\
TEST GROUP: (ICS + F12) ICS 400-800 mcg/day + formoterol 12 mcg BID \\
CONTROL GROUP: (ICS) ICS (400-800 mcg/day) + placebo BID \\
DEVICE: MDI + large volume spacer (Volumatic) \\
NUMBER OF DEVICES: 2 \\
COMPLIANCE: assessed by weighing canisters \\
CO-TREATMENT: prn SABA \\
\hline Interventions \\
\end{tabular}


Outcomes

\begin{tabular}{|c|c|c|}
\hline Outcomes & \multicolumn{2}{|c|}{$\begin{array}{l}\text { PULMONARY FUNCTION TEST: FEV1 predicted; am PEFR; pm PEF; PEF } \\
\text { variability (\%); PC } 20 \\
\text { SYMPTOM SCORES: score of } 0 \text { to } 3 \text { (max 9); nighttime symptom score; } \\
\text { symptom-free days or nights } \\
\text { FUNCTIONAL STATUS: Rescue medication use; exacerbation requiring } \\
\text { systemic steroids; exacerbations requiring admission } \\
\text { INFLAMMATORY MARKERS: not described } \\
\text { ADVERSE EFFECTS: described } \\
\text { WITHDRAWALS: not described } \\
\text { primary outcome measure not reported }\end{array}$} \\
\hline Notes & \multicolumn{2}{|c|}{$\begin{array}{l}\text { Full-text publication } \\
\text { Funded by Astra Zeneca } \\
\text { Author contacted and unable to confirm methodology or data } \\
\text { User-defined number: } 600 \text { (mean ICS dose in LAB2 group in mcg/day of BDP- } \\
\text { equivalent: } 400 \text { - 800) }\end{array}$} \\
\hline \multicolumn{3}{|l|}{ Risk of bias } \\
\hline Item & Authors' judgement & Description \\
\hline Adequate sequence generation? & Unclear & $\begin{array}{l}\text { Described as randomised; no other information } \\
\text { presented }\end{array}$ \\
\hline Allocation concealment? & Unclear & Information not available \\
\hline $\begin{array}{l}\text { Blinding? } \\
\text { All outcomes }\end{array}$ & Yes & Double-blind; identical placebo used \\
\hline $\begin{array}{l}\text { Incomplete outcome data } \\
\text { addressed? } \\
\text { All outcomes }\end{array}$ & Unclear & $\begin{array}{l}\text { No information available on the statistical handling of } \\
\text { missing data }\end{array}$ \\
\hline Free of selective reporting? & Yes & Data on OCS-treated exacerbations available \\
\hline Free of other bias? & Unclear & $\begin{array}{l}\text { No data provided on } \% \text { participants meeting eligibility } \\
\text { criteria from screening or run-in populations }\end{array}$ \\
\hline
\end{tabular}

Bisgaard 2006 
CO-TREATMENT: prn SABA

\begin{tabular}{|c|c|c|}
\hline Outcomes & \multicolumn{2}{|c|}{$\begin{array}{l}\text { PULMONARY FUNCTION TEST: Recorded but not reported } \\
\text { SYMPTOM SCORES: Recorded but not reported } \\
\text { FUNCTIONAL STATUS: Night awakenings; rescue medication use; } \\
\text { exacerbations* (hospitalisation/need for OCS or other medication, increase in } \\
\text { ICS, PEF >I=70\% baseline on two consecutive days) } \\
\text { INFLAMMATORY MARKERS: Not reported } \\
\text { ADVERSE EFFFECTS: Reported } \\
\text { WITHDRAWAL: Not reported } \\
\text { *primary outcome }\end{array}$} \\
\hline Notes & \multicolumn{2}{|c|}{$\begin{array}{l}\text { Full text article } \\
\text { Additional data downloaded from AZ website } \\
\text { (www.astrazenecaclinicaltrials.com) } \\
\text { Funded by AstraZeneca } \\
\text { User defined: } 320\end{array}$} \\
\hline \multicolumn{3}{|l|}{ Risk of bias } \\
\hline Item & Authors' judgement & Description \\
\hline Adequate sequence generation? & Yes & Computer generated randomisation scheme \\
\hline Allocation concealment? & Unclear & $\begin{array}{l}\text { Eligible patients were randomized in balanced blocks by } \\
\text { allocating patient numbers in consecutive order }\end{array}$ \\
\hline $\begin{array}{l}\text { Blinding? } \\
\text { All outcomes }\end{array}$ & Yes & Double-blind; identical inhaler devices used. \\
\hline $\begin{array}{l}\text { Incomplete outcome data } \\
\text { addressed? } \\
\text { All outcomes }\end{array}$ & Unclear & $\begin{array}{l}\text { 'All analyses were performed on an intention-to-treat } \\
\text { basis.' Additional information on the composition of the } \\
\text { ITT population was not provided }\end{array}$ \\
\hline Free of selective reporting? & Yes & $\begin{array}{l}\text { Data on OCS-treated exacerbations reported as } \\
\text { composite with ED visits/hospitalisations, PEF falls and } \\
\text { requirement for medical intervention. Request for } \\
\text { separate data on OCS-treated exacerbations from study } \\
\text { sponsors has not been successful }\end{array}$ \\
\hline Free of other bias? & Unclear & $\begin{array}{l}\text { No data provided on } \% \text { participants meeting eligibility } \\
\text { criteria from screening or run-in populations }\end{array}$ \\
\hline
\end{tabular}

Heuck 2000

\begin{tabular}{ll}
\hline Methods & Crossover study; single centre (outpatient referral centre). \\
\hline Participants & Asthmatic Children \\
\%ELIGIBLE OF SCREENED POPULATION: Not reported \\
\%RUN IN PARTICIPANTS RANDOMISED: Not reported \\
RANDOMISED: 27 \\
WITHDRAWALS: 2 patients were withdrawn during treatment with budesonide \\
alone 1 withdrawal - unclear which period \\
MEAN AGE (RANGE): 9.6 (6.1-13.5) \\
GENDER (\% male): 52 \\
SEVERITY: Mild to moderate \\
BASELINE FEV1: Not reported \\
BASELINE PEF L/min (range): $2801 /$ min \\
BASELINE DOSE OF ICS: Mean (range) - BUD 200 bid or equivalent \\
ASTHMA DURATION (range in years): 4.5 (1.4-9.5) \\
ATOPY (\%): Not reported \\
ELIGIBILITY CRITERIA: Treatment with inhaled budesonide 200 ?g twice daily \\
(or equipotent doses of other ICS) for one month before study entry; children were \\
prepubertal \\
EXCLUSION CRITERIA: Not described \\
LABA + ICS versus INCREASED DOSE ICS \\
OUTCOMES: reported weekly \\
RUN-IN: None \\
DOSE OF ICS DURING RUN IN: N/A \\
INTERVENTION PERIOD: 12 weeks \\
TEST GROUP: (Form + ICS) Formoterol 12 mcg bid + Budesonide 100 bid \\
\hline Interventions
\end{tabular}

Cochrane Database Syst Rev. Author manuscript; available in PMC 2014 September 19. 
CONTROL GROUP: Placebo + ICS Budesonide 200 bid

DEVICE: Turbuhaler (ICS) and Aerolizer (formoterol)

NUMBER OF DEVICES: 2

COMPLIANCE: Turbuhalers weighed and number of formoterol capsules counted CO-TREATMENT: prn SABA

\begin{tabular}{|c|c|c|}
\hline Outcomes & \multicolumn{2}{|c|}{$\begin{array}{l}\text { PULMONARY FUNCTION TEST: FEV1; am PEF; pm PEF } \\
\text { SYMPTOM SCORE: Daytime and night time score (score of } 0 \text { to 4) } \\
\text { FUNCTIONAL STATUS Exacerbations; rescue medication use; lower leg growth; } \\
\text { serum and urinary markers of type I and III collagen turnover } \\
\text { INFLAMMATORY MARKERS: Inflammatory markers in serum } \\
\text { ADVERSE EFFECTS: Reported } \\
\text { WITHDRAWALS: Reported } \\
\text { Primary outcome measure: Not reported }\end{array}$} \\
\hline Notes & \multicolumn{2}{|c|}{$\begin{array}{l}\text { Full text publication } \\
\text { Source of funding not stated } \\
\text { Confirmation of methodology and data not obtained } \\
\text { User-defined number: } 200 \text { (400-200) }\end{array}$} \\
\hline \multicolumn{3}{|l|}{ Risk of bias } \\
\hline Item & Authors' judgement & Description \\
\hline Adequate sequence generation? & Yes & $\begin{array}{l}\text { 'Treatment order was allocated by a } \\
\text { computerised randomisation scheme } \\
\text { prepared in balanced blocks.' }\end{array}$ \\
\hline Allocation concealment? & Unclear & $\begin{array}{l}\text { Information on concealment of allocation not } \\
\text { provided }\end{array}$ \\
\hline $\begin{array}{l}\text { Blinding? } \\
\text { All outcomes }\end{array}$ & Yes & Double-blind; double dummy \\
\hline $\begin{array}{l}\text { Incomplete outcome data } \\
\text { addressed? } \\
\text { All outcomes }\end{array}$ & Unclear & No information available \\
\hline Free of selective reporting? & Yes & Data on OCS-treated exacerbations available \\
\hline Free of other bias? & Unclear & $\begin{array}{l}\text { No data provided on } \% \text { participants meeting } \\
\text { eligibility criteria from screening or run-in } \\
\text { populations }\end{array}$ \\
\hline
\end{tabular}

Langton Hewer 1995

\begin{tabular}{ll}
\hline Methods & Parallel group, single centre study \\
\hline Participants & Symptomatic children \\
\%ELIGIBLE OF SCREENED POPULATION: Not reported \\
\% RUN IN PARTICIPANTS RANDOMISED: Not reported \\
NUMBER RECRUITED NOT RANDOMISED: Not stated \\
RANDOMISED: 23 (usual ICS + Sal 100 bid: 11; usual ICS: 12) \\
WITHDRAWALS: Usual ICS + S: 0; usual ICS: 2 \\
AGE median (range) years: 15 (12-17) \\
GENDER:(\% male): 70 \\
SEVERITY: Severe \\
BASELINE \% PRED. FEV1: 82 \\
BASELINE DOSE OF ICS (start of run in): 400 \\
ASTHMA DURATION: 13 years \\
ATOPY (\%): 100\% \\
ELIGIBILITY CRITERIA: Severe asthma (not defined but severe enough to be \\
attending residential school for asthma and persistent symptoms) \\
EXCLUSION CRITERIA: Already on LABA; \\
CRITERIA FOR RANDOMISATION DURING RUN-IN: None specified \\
\hline LABA + ICS versus SAME DOSE (usual dose) of ICS \\
OUTCOMES reported at 8 \& 10 weeks \\
RUN IN PERIOD: 2 weeks \\
DOSE OF ICS DURING RUN IN: Same as during study \\
DOSE OPTIMISATION PERIOD: None \\
INTERVENTION PERIOD: 8 weeks
\end{tabular}

Cochrane Database Syst Rev. Author manuscript; available in PMC 2014 September 19. 
TEST GROUP: (Usual ICS+S): Salmeterol 100mcg bid

CONTROL GROUP: Usual ICS and placebo bid

DEVICE: diskhaler

NUMBER OF DEVICES: 2

COMPLIANCE: Supervised in school taking medication by investigators

CO-TREATMENT oral steroids, methylxanthines \& anticholinergics taken by $20 \%$ participants

\begin{tabular}{|c|c|c|}
\hline Outcomes & \multicolumn{2}{|c|}{$\begin{array}{l}\text { PULMONARY FUNCTION TEST: FEV1; am PEF; pm PEF } \\
\text { SYMPTOM SCORES: Morning and evening symptom scores } \\
\text { FUNCTIONAL STATUS: Rescue B2-agonist; symptom-free days/nights; } \\
\text { exacerbation (requiring systemic steroids); quality of life score } \\
\text { INFLAMMATORY MARKERS: none } \\
\text { ADVERSE EFFECTS: described } \\
\text { WITHDRAWALS: described } \\
\text { Primary outcome measure not reported }\end{array}$} \\
\hline Notes & \multicolumn{2}{|c|}{$\begin{array}{l}\text { Full-text publication } \\
\text { Funded by Charity } \\
\text { Confirmation of methodology and data pending } \\
\text { User-defined number: not reported }\end{array}$} \\
\hline \multicolumn{3}{|l|}{ Risk of bias } \\
\hline Item & Authors' judgement & Description \\
\hline Adequate sequence generation? & Unclear & Described as randomised; no other information presented \\
\hline Allocation concealment? & Unclear & Information not available \\
\hline $\begin{array}{l}\text { Blinding? } \\
\text { All outcomes }\end{array}$ & Yes & Identical placebo \\
\hline $\begin{array}{l}\text { Incomplete outcome data } \\
\text { addressed? } \\
\text { All outcomes }\end{array}$ & Unclear & $\begin{array}{l}\text { Data analysis described as intention to treat; methods } \\
\text { applied not elaborated }\end{array}$ \\
\hline Free of selective reporting? & Yes & Data on OCS-treated exacerbations available \\
\hline Free of other bias? & Unclear & $\begin{array}{l}\text { No data provided on \% participants meeting eligibility } \\
\text { criteria from screening or run-in populations (niche } \\
\text { population sampled for residential school for children } \\
\text { with particularly difficult to treat asthma) }\end{array}$ \\
\hline
\end{tabular}

Malone 2005

\begin{tabular}{ll}
\hline Methods & Parallel group, multicentre (66 centres in North America) \\
\hline Participants & Steroid-using asthmatic children \\
\%ELIGIBLE OF SCREENED POPULATION: Not reported \\
\%RUN-IN PARTICIPANTS RANDOMISED: 48 \\
RANDOMISED: 203 (FP/SAL: 101; FP: 102) \\
WITHDRAWAL: FP/SAL: 19; FP: 16 \\
AGE mean: 8 years \\
GENDER (male\%): 64 \\
ASTHMA SEVERITY: Mild-moderate \\
BASELINE \% PRED. FEV1 mean: 80 \\
BASELINE DOSE OF ICS (start of run in): 166mcg (FP stratum) \\
ASTHMA DURATION: Not reported \\
ATOPY(\%): Not reported \\
ELIGIBILITY CRITERIA: 4-11 years; ATS defined asthma for at least 2 months; \\
ICS therapy (BDP equivalent 252-336mcg/d) for 1 month prior to entry; \\
participants aged 6-11 required to have FEV1\% predicted; participants aged 4-5 \\
required to have am PEF 50-95\% predicted; >/=12\% response to beta-agonist at \\
screening visit or within one year of screening visit \\
EXCLUSION CRITERIA: History of life-threatening asthma; hospitalisation with \\
asthma twice or more in previous year; significant concurrent disease; oral or \\
parenteral use of steroids in month prior to study entry \\
CRITERIA FOR RANDOMISATION DURING RUN-IN: am FEV1 50-95\% \\
predicted; daytime asthma (score at least 1)/use of SABA on 3+ days of last 7 days \\
of run-in; 70\% or greater diary card entry \\
\hline
\end{tabular}




\begin{tabular}{|c|c|c|}
\hline Interventions & \multicolumn{2}{|c|}{$\begin{array}{l}\text { LABA + ICS versus SAME dose of ICS } \\
\text { OUTCOMES: Reported at } 3 \text { months } \\
\text { RUN IN PERIOD: } 2 \text { weeks } \\
\text { DOSE OF ICS DURING RUN IN: Usual maintenance dose } \\
\text { INTERVENTION PERIOD: } 3 \text { months } \\
\text { TEST GROUP: Combination Salmeterol 50/Fluticasone 100mcg bid } \\
\text { CONTROL GROUP: Fluticasone 100mcg bid } \\
\text { DEVICE: Diskus } \\
\text { NUMBER OF DEVICES: } 1 \\
\text { COMPLIANCE: Not reported } \\
\text { CO-TREATMENT: prn SABA }\end{array}$} \\
\hline Outcomes & \multicolumn{2}{|c|}{$\begin{array}{l}\text { PULMONARY FUNCTION TEST: FEV1; Clinic PEF; am PEF; pm PEF } \\
\text { SYMPTOM SCORES: Symptom scores; symptom-free days } \\
\text { FUNCTIONAL STATUS: OCS-treated exacerbations; hospitalisations; Use of } \\
\text { reliever medication; SABA-free days } \\
\text { INFLAMMATORY MARKERS: Not reported } \\
\text { ADVERSE EFFECTS: Reported } \\
\text { WITHDRAWAL: Stated } \\
\text { * primary outcome: not identified (safety study) }\end{array}$} \\
\hline Notes & \multicolumn{2}{|c|}{$\begin{array}{l}\text { Full-text publication } \\
\text { Funded by GSK } \\
\text { User-defined number: } 400 \\
\text { Confirmation of data and methodology obtained }\end{array}$} \\
\hline \multicolumn{3}{|l|}{ Risk of bias } \\
\hline Item & Authors' judgement & Description \\
\hline Adequate sequence generation? & Yes & See Appendix 1 \\
\hline Allocation concealment? & Yes & See Appendix 1 \\
\hline $\begin{array}{l}\text { Blinding? } \\
\text { All outcomes }\end{array}$ & Yes & Double-blind; identical inhaler devices \\
\hline $\begin{array}{l}\text { Incomplete outcome data } \\
\text { addressed? } \\
\text { All outcomes }\end{array}$ & No & $\begin{array}{l}\text { 'For patients who withdrew from the } \\
\text { study prematurely, all available data } \\
\text { up to the time of discontinuation were } \\
\text { included in the intent-to-treat } \\
\text { population.' }\end{array}$ \\
\hline Free of selective reporting? & Yes & $\begin{array}{l}\text { Data on OCS treated exacerbations } \\
\text { and hospital admission available on } \\
\text { request }\end{array}$ \\
\hline Free of other bias? & Yes & $\begin{array}{l}48 \% \text { of screening population eligible } \\
\text { for randomisation }\end{array}$ \\
\hline
\end{tabular}

Meijer 1995

\begin{tabular}{ll}
\hline Methods & Parallel group, single centre study \\
\hline Participants & Asymptomatic asthmatic children \\
\%ELIGIBLE OF SCREENED POPULATION: Not reported \\
\%RUN-IN PARTICIPANTS RANDOMISED: Not reported \\
RANDOMISED: 40 (Sal 50 mcg BID + ICS: 20; ICS + Placebo: 20) \\
WITHDRAWALS: Sal50 mcg BID + ICS: 0; ICS + Placebo: 1 (5\%) \\
AGE: mean (SD): 11.4 (2.6) \\
GENDER (\%male): 58 \\
SEVERITY: Mild \\
BASELINE \% PRED. FEV1: 94 \\
BASELINE DOSE OF ICS: Twice daily 200 or 400 mcg beclomethasone \\
dipropionate rotadisk \\
ASTHMA DURATION: 8.4 years \\
ATOPY (\%): 100 \\
ELIGIBILITY CRITERIA: None reported \\
EXCLUSION CRITERIA: None reported \\
CRITERIA FOR RANDOMISATION DURING RUN IN: N/A
\end{tabular}

Interventions

LABA + ICS versus SAME dose of ICS 
OUTCOMES: reported at $1,8,16$ weeks

RUN IN PERIOD: None

DOSE OPTIMISATION PERIOD: None

INTERVENTION PERIOD: 16 weeks

TEST GROUP: Salmeterol $50 \mathrm{mcg}$ BID + BDP $250 \mathrm{mcg}$ BID

CONTROL GROUP: BDP $250 \mathrm{mcg}$ BID + placebo

DEVICE: dry powder inhaler (diskhaler)

NUMBER OF DEVICES: 2

COMPLIANCE: Returned powder disks counted

CO-TREATMENT: prn SABA

\begin{tabular}{|c|c|c|}
\hline Outcomes & \multicolumn{2}{|c|}{$\begin{array}{l}\text { PULMONARY FUNCTION TEST: FEV1 predicted; PC20 doubling doses (DD); } \\
\text { circadian variation (day-night differences in FEV1) } \\
\text { SYMPTOM SCORES: Only individual symptoms reported (yes/no) } \\
\text { FUNCTIONAL STATUS: Rescue medication use } \\
\text { INFLAMMATORY MARKERS: Not reported } \\
\text { ADVERSE EFFECTS: Not reported } \\
\text { WITHDRAWALS: Reported } \\
\text { Primary outcome not specified }\end{array}$} \\
\hline Notes & \multicolumn{2}{|c|}{$\begin{array}{l}\text { Full-text publication } \\
\text { Funded by Glaxo } \\
\text { User-defined number: } 500 \\
\text { Confirmation of data and methodology not obtained }\end{array}$} \\
\hline \multicolumn{3}{|l|}{ Risk of bias } \\
\hline Item & Authors' judgement & Description \\
\hline Adequate sequence generation? & Unclear & $\begin{array}{l}\text { Described as randomised; no other } \\
\text { information presented }\end{array}$ \\
\hline Allocation concealment? & Unclear & Information not available \\
\hline $\begin{array}{l}\text { Blinding? } \\
\text { All outcomes }\end{array}$ & Yes & Double-blind; double dummy \\
\hline $\begin{array}{l}\text { Incomplete outcome data } \\
\text { addressed? } \\
\text { All outcomes }\end{array}$ & Unclear & $\begin{array}{l}\text { Not enough information presented to } \\
\text { determine this }\end{array}$ \\
\hline Free of selective reporting? & Unclear & $\begin{array}{l}\text { Unclear whether data on OCS-treated } \\
\text { exacerbations were collected in the study }\end{array}$ \\
\hline Free of other bias? & Unclear & $\begin{array}{l}\text { No data presented on } \% \text { screening population } \\
\text { eligible for randomisation }\end{array}$ \\
\hline
\end{tabular}

Morice 2008a

\begin{tabular}{ll}
\hline Methods & $\begin{array}{l}\text { Parallel group, multicentre study (53 centres in South America, Europe, Hong Kong } \\
\text { and Taiwan) }\end{array}$ \\
\hline Participants & \% ELIGIBLE OF SCREENED POPULATION: Not reported \\
\% RUN-IN PARTICIPANTS RANDOMISED: 77 \\
RANDOMISED: 622 (BUD: 207; BUD/F (dpi): 203; BUD/F (mdi): 212) \\
WITHDRAWALS: BUD: 14 BUD/F (dpi): 11; BUD/F (mdi): 14 \\
AGE: mean (range): 9 (6-11 years) \\
GENDER (\%male): 66 \\
SEVERITY: Not specified \\
BASELINE \% PRED. FEV1: 89 \\
BASELINE DOSE OF ICS: (start of run-in): 470mcg \\
ASTHMA DURATION: Not reported \\
ATOPY (\%): Not reported \\
ELIGIBILITY CRITERIA: Age 6-11 years; diagnosis of asthma for at least 6 \\
months; PEF >50\% of predicted normal; history daily ICS use (stable dose of \\
375-1000 mcg 30 days prior to enrolment); clinically important exercise-induced \\
bronchoconstriction for 3 months before enrolment; ability to use DPI, pMDI \& \\
peak flow meter \\
EXCLUSION CRITERIA: Not reported \\
CRITERIA FOR RANDOMISATION DURING RUN-IN: Symptom score 1-4; \\
mean morning PEF 50-85\% post-SABA \\
\hline
\end{tabular}




\begin{tabular}{|c|c|c|}
\hline Interventions & \multicolumn{2}{|c|}{$\begin{array}{l}\text { OUTCOMES: } 12 \text { weeks } \\
\text { RUN-IN PERIOD: } 2 \text { weeks } \\
\text { DOSE OF ICS DURING RUN-IN: } 470 \\
\text { DOSE OPTIMISATION PERIOD: Not reported } \\
\text { INTERVENTION PERIOD: } 12 \text { weeks } \\
\text { TEST GROUP: Combination formoterol and budesonide (160/9mcg) BID via dry } \\
\text { powder inhaler + placebo metered dose inhaler } \\
\text { CONTROL GROUP: Budesonide 100mcg BID } \\
\text { DEVICE: BUD/F mdi and Budesonide: MDI; BUD/F dpi: DPI } \\
\text { NUMBER OF DEVICES: } 2 \\
\text { COMPLIANCE: Not reported } \\
\text { CO-TREATMENT: prn SABA }\end{array}$} \\
\hline Outcomes & \multicolumn{2}{|c|}{$\begin{array}{l}\text { PULMONARY FUNCTION TEST: Am PEF*; pm PEF; FEV1 } \\
\text { SYMPTOM SCORES: Day/night scores } \\
\text { FUNCTIONAL STATUS: Paediatric AQLQ } \\
\text { INFLAMMATORY MARKERS: NA } \\
\text { ADVERSE EFFECTS: Stated } \\
\text { WITHDRAWALS: Stated } \\
\text { Primary outcome measure* }\end{array}$} \\
\hline Notes & \multicolumn{2}{|c|}{$\begin{array}{l}\text { Full text publication } \\
\text { AZ funded } \\
\text { User defined: } 200 \\
\text { Confirmation of data and methodology not obtained }\end{array}$} \\
\hline \multicolumn{3}{|l|}{ Risk of bias } \\
\hline Item & Authors' judgement & Description \\
\hline Adequate sequence generation? & Yes & Computer-generated schedule \\
\hline Allocation concealment? & Unclear & Information not provided \\
\hline $\begin{array}{l}\text { Blinding? } \\
\text { All outcomes }\end{array}$ & Yes & Double-blind; double dummy \\
\hline $\begin{array}{l}\text { Incomplete outcome data } \\
\text { addressed? } \\
\text { All outcomes }\end{array}$ & Unclear & $\begin{array}{l}\text { '...all randomised patients with post- } \\
\text { randomisation data.' }\end{array}$ \\
\hline Free of selective reporting? & No & $\begin{array}{l}\text { Data on OCS-treated exacerbations were not } \\
\text { reported in the trial publication. Study } \\
\text { sponsors have indicated that the data from } \\
\text { this study are not available }\end{array}$ \\
\hline Free of other bias? & Yes & $\begin{array}{l}77 \% \text { of screening population eligible for } \\
\text { randomisation }\end{array}$ \\
\hline
\end{tabular}

Morice 2008b

\begin{tabular}{lll}
\hline Methods & See Morice 2008a \\
\hline Participants & See Morice 2008a \\
\hline Interventions & $\begin{array}{l}\text { See Morice 2008a, except for: } \\
\text { TEST GROUP: } \\
\text { Combination formoterol and budesonide (160/9mcg) BID via metered dose } \\
\text { inhaler + placebo dry powder inhaler }\end{array}$ \\
\hline Outcomes & See Morice 2008a \\
\hline Notes & See Morice 2008a & \\
\hline Risk of bias & Description \\
\hline Item & Authors' judgement & See Morice 2008a \\
\hline Adequate sequence generation? & Yes & See Morice 2008a \\
\hline Allocation concealment? & Unclear & \\
\hline
\end{tabular}

Cochrane Database Syst Rev. Author manuscript; available in PMC 2014 September 19. 


\begin{tabular}{lll}
$\begin{array}{l}\text { Blinding? } \\
\text { All outcomes }\end{array}$ & Yes & See Morice 2008a \\
\hline $\begin{array}{l}\text { Incomplete outcome data addressed? } \\
\text { All outcomes }\end{array}$ & Unclear & See Morice 2008a \\
\hline Free of selective reporting? & No & See Morice 2008a \\
\hline Free of other bias? & Yes & See Morice 2008a \\
\hline
\end{tabular}

Ortega-Cisneros 1998

\begin{tabular}{|c|c|c|}
\hline Methods & \multicolumn{2}{|c|}{ Parallel-group, single centre } \\
\hline Participants & \multicolumn{2}{|c|}{$\begin{array}{l}\text { Symptomatic Asthmatic children } \\
\text { \%ELIGIBLE OF SCREENED POPULATION: Not reported } \\
\text { \%RUN IN PARTICAPNTS RANDOMISED: Not reported } \\
\text { RANDOMISED: S50 + BDP: 10; BDP: } 10 \\
\text { WITHDRAWALS: Not described } \\
\text { AGE (range): } 6 \text { to } 19 \text { years } \\
\text { GENDER (\% male): Not described } \\
\text { SEVERITY: Moderate } \\
\text { BASELINE \% PRED. FEV1 mean (SD): Not described } \\
\text { ASTHMA DURATION: Not reported } \\
\text { ATOPY(\%): Not reported } \\
\text { ELIGIBILITY CRITERIA: Still symptomatic despite maintenance treatment } \\
\text { with } 200 \text { mcg bid of BDP } \\
\text { EXCLUSION CRITERIA: Not described }\end{array}$} \\
\hline Interventions & \multicolumn{2}{|c|}{$\begin{array}{l}\text { LABA + ICS versus INCREASED dose } \\
\text { ICS RUN-IN PERIOD: } 2 \text { weeks } \\
\text { OUTCOMES: Reported at } 8,12 \text { weeks } \\
\text { DOSE OF ICS DURING RUN-IN: BDP } 200 \text { bid } \\
\text { DOSE OPTIMISATION PERIOD: None } \\
\text { INTERVENTION PERIOD: } 12 \text { weeks } \\
\text { TEST GROUP: Salmeterol } 50 \text { mcg bid + beclomethasone 200mcg bid } \\
\text { CONTROL GROUP: Beclomethasone } 400 \text { bid } \\
\text { DEVICE: Not specified } \\
\text { NUMBER OF DEVICES: } 2 \\
\text { COMPLIANCE: Not reported } \\
\text { CO-TREATMENT: Not specified }\end{array}$} \\
\hline Outcomes & \multicolumn{2}{|c|}{$\begin{array}{l}\text { PULMONARY FUNCTION TEST: FEV1; PEF; FEF 25-75\% } \\
\text { SYMPTOM SCORES: Symptoms } \\
\text { FUNCTIONAL STATUS: Not reported } \\
\text { INFLAMMATORY MARKERS: Not reported } \\
\text { ADVERSE EFFECTS: Not reported } \\
\text { WITHDRAWAL: Not reported }\end{array}$} \\
\hline Notes & \multicolumn{2}{|c|}{$\begin{array}{l}\text { Abstract } \\
\text { Funding not reported } \\
\text { Confirmation of methodology and data extraction: not obtained } \\
\text { User-defined order: } 400\end{array}$} \\
\hline \multicolumn{3}{|l|}{ Risk of bias } \\
\hline Item & Authors' judgement & Description \\
\hline Adequate sequence generation? & Unclear & $\begin{array}{l}\text { Described as randomised; no other information } \\
\text { presented }\end{array}$ \\
\hline Allocation concealment? & Unclear & Information not provided \\
\hline $\begin{array}{l}\text { Blinding? } \\
\text { All outcomes }\end{array}$ & No & Open label \\
\hline $\begin{array}{l}\text { Incomplete outcome data } \\
\text { addressed? } \\
\text { All outcomes }\end{array}$ & Unclear & No information provided \\
\hline
\end{tabular}




\begin{tabular}{lll} 
Free of selective reporting? & Unclear & $\begin{array}{l}\text { Unclear whether data on OCS-treated exacerbations } \\
\text { were collected in the study }\end{array}$ \\
\hline Free of other bias? & Unclear & No information provided \\
\hline
\end{tabular}

Pohunek 2006a

\begin{tabular}{|c|c|}
\hline Methods & Parallel group, multicentre study ( 80 centres in Europe). Three treatment groups \\
\hline Participants & $\begin{array}{l}\text { Steroid-using asthmatic children } \\
\text { \%ELIGIBLE OF SCREENED POPULATION: Not reported } \\
\text { \%RUN-IN PARTICIPANTS RANDOMISED: } 77 \\
\text { RANDOMISED: } 429 \text { (BUD/F: 216; BUD: } 213 \text { ) } \\
\text { WITHDRAWAL: BUD/F: } 14 \text { BUD: } 13 \\
\text { AGE mean (range) } 8 \text { (4-11) } \\
\text { GENDER (male\%): } 67 \\
\text { ASTHMA SEVERITY: Mild-moderate } \\
\text { BASELINE \% PRED. FEV1 mean: } 92 \% \\
\text { BASELINE DOSE OF ICS (start of run in): } 454 \mathrm{mcg} / \mathrm{d} \\
\text { ASTHMA DURATION: } 3 \\
\text { ATOPY(\%): Not reported } \\
\text { ELIGIBILITY CRITERIA: } 4-11 \text { years; diagnosis of asthma (ATS) for a minimum } \\
\text { period of } 6 \text { months; pre-SABA PEF >/ = 50\% predicted; ICS treatment for at least } \\
12 \text { weeks before entry into the study, at a constant dose of } 375 ? 1000 \text { mcg/d during } \\
\text { the } 30 \text { days prior to enrolment; History an average of }>/=1 \text { clinically important } \\
\text { exercise induced bronchoconstriction per week during the } 12 \text { weeks months leading } \\
\text { up to the study; ability to use Turbuhaler device \& peak flow metre } \\
\text { EXCLUSION CRITERIA: Oral, parenteral or rectal corticosteroids within } 30 \text { days; } \\
\text { respiratory infection affecting asthma control within } 30 \text { days; any significant } \\
\text { coexisting disease/disorder; known/suspected hypersensitivity to study medication } \\
\text { or inhaled lactose; inhaled anticholinergics, beta-blockers (including eye drops), } \\
\text { xanthines \& other anti-asthma agents not permitted during the study } \\
\text { POST-RUN IN: Total asthma-symptom score of at least one on a minimum of four } \\
\text { of last } 7 \text { days of the run-in period; during last } 7 \text { days of the run-in, patients had to } \\
\text { have a mean morning PEF of 50-85\% of the post-SABA PEF }\end{array}$ \\
\hline Interventions & $\begin{array}{l}\text { LABA + ICS versus SAME dose ICS } \\
\text { OUTCOMES: } 12 \text { weeks } \\
\text { RUN IN PERIOD: } 10-14 \text { days } \\
\text { DOSE OF ICS DURING RUN IN: Usual dose of ICS } \\
\text { INTERVENTION PERIOD: } 12 \text { weeks } \\
\text { TEST GROUP: Combination Budesonide/Formoterol 200/6mcg bid } \\
\text { CONTROL GROUP: Budesonide } 200 \mathrm{mcg} \text { bid } \\
\text { DEVICE: Turbuhaler } \\
\text { NUMBER OF DEVICES: } 2 \text { (double dummy) } \\
\text { COMPLIANCE: Not reported } \\
\text { CO-TREATMENT: prn SABA }\end{array}$ \\
\hline Outcomes & $\begin{array}{l}\text { PULMONARY FUNCTION TEST: Am PEF; pm PEF; FEV1 } \\
\text { SYMPTOM SCORES: Not reported } \\
\text { FUNCTIONAL STATUS: Not reported } \\
\text { INFLAMMATORY MARKERS: Not reported } \\
\text { ADVERSE EFFECTS: Reported } \\
\text { WITHDRAWAL: Not reported } \\
\text { primary outcome: not reported }\end{array}$ \\
\hline Notes & $\begin{array}{l}\text { Full-text publication } \\
\text { Funded by AstraZeneca } \\
\text { Confirmation of methodology and data: Not obtained } \\
\text { User-defined order: } 400\end{array}$ \\
\hline \multicolumn{2}{|l|}{ Risk of bias } \\
\hline Item & Authors' judgement \\
\hline Adequate sequence generation? & $\begin{array}{l}\text { Schedule generated using a computer } \\
\text { program (AstraZeneca, UK) }\end{array}$ \\
\hline Allocation concealment? & $\begin{array}{l}\text { Person not involved in the study team } \\
\text { generated the randomisation schedule }\end{array}$ \\
\hline
\end{tabular}




\begin{tabular}{lll}
$\begin{array}{l}\text { Blinding? } \\
\text { All outcomes }\end{array}$ & Yes & Double-blind; double dummy \\
\hline $\begin{array}{l}\text { Incomplete outcome data } \\
\text { addressed? } \\
\text { All outcomes }\end{array}$ & Unclear & $\begin{array}{l}\text { 'Intent to treat analysis was performed using } \\
\text { data from all randomized patients.' } \\
\text { No additional data were provided on the } \\
\text { composition of the ITT population }\end{array}$ \\
\hline Free of selective reporting? & Unclear & $\begin{array}{l}\text { Unclear whether OCS-treated exacerbations } \\
\text { were collected in the study. Correspondence } \\
\text { with trialist has failed to clarify this }\end{array}$ \\
\hline Free of other bias? & Yes & $\begin{array}{l}78 \% \text { screening population eligible for } \\
\text { randomisation }\end{array}$ \\
\hline
\end{tabular}

Pohunek 2006b

\begin{tabular}{|c|c|c|}
\hline Methods & \multicolumn{2}{|l|}{ See Pohunek 2006a } \\
\hline Participants & \multicolumn{2}{|c|}{$\begin{array}{l}\text { See Pohunek 2006a, except for } \\
\text { RANDOMISED: } 414 \text { (F + BUD: 201; Bud: 213) }\end{array}$} \\
\hline Interventions & \multicolumn{2}{|c|}{$\begin{array}{l}\text { See Pohunek 2006a, except for } \\
\text { TEST GROUP: Separate Formoterol } 6 \text { and Budesonide } 200 \mathrm{mcg} \text { bid } \\
\text { NUMBER OF DEVICES: } 2\end{array}$} \\
\hline Outcomes & \multicolumn{2}{|l|}{ See Pohunek 2006a } \\
\hline Notes & \multicolumn{2}{|l|}{ See Pohunek 2006a } \\
\hline \multicolumn{3}{|l|}{ Risk of bias } \\
\hline Item & Authors' judgement & Description \\
\hline Adequate sequence generation? & Yes & See Pohunek 2006a \\
\hline Allocation concealment? & Yes & See Pohunek 2006a \\
\hline $\begin{array}{l}\text { Blinding? } \\
\text { All outcomes }\end{array}$ & Yes & See Pohunek 2006a \\
\hline $\begin{array}{l}\text { Incomplete outcome data addressed? } \\
\text { All outcomes }\end{array}$ & Unclear & See Pohunek 2006a \\
\hline Free of selective reporting? & Unclear & See Pohunek 2006a \\
\hline Free of other bias? & Yes & See Pohunek 2006a \\
\hline
\end{tabular}

Russell 1995

\begin{tabular}{ll}
\hline Methods & Parallel-group, multicentre study (78 centres) \\
\hline Participants & Symptomatic Asthmatic Children \\
\%ELIGIBLE OF SCREENED POPULATION: Not reported \\
\%RUN-IN PARTICIPANTS RANDOMISED: Not reported \\
RANDOMISED: 208 (Salm50 + ICS: 99; Placebo + ICS: 109) \\
WITHDRAWALS: Salm50 + ICS: 22\%; Placebo + ICS: 16.8\% \\
AGE: mean (SD): $10.2(2.7)$ \\
GENDER: $\%$ male): 60 \\
SEVERITY: Moderate \\
BASELINE MEAN \% PRED. FEV1: 78 \\
BASELINE DOSE OF ICS: 750 mcg \\
ASTHMA DURATION (\%): <1 year: $3 ; 1$ to 5 years: $20 ;>5$ years: 77 \\
ATOPY(\%): 77 \\
ELIGIBILITY CRITERIA DURING RUN-IN: Morning PEF-PP(percent predicted) \\
<=90 on 4 or more days of the last 10 days of the baseline period; either recorded \\
symptoms on at least 7 of 14 days of the baseline period for which they used at least
\end{tabular}

Cochrane Database Syst Rev. Author manuscript; available in PMC 2014 September 19. 
one salbutamol blister per episode; recorded a diurnal variation in PEF of $>=15 \%$ on at least 7 occasions during baseline period

EXCLUSION CRITERIA: Received a course of oral corticosteroids; change in prophylactic therapy during the previous 2 weeks

\begin{tabular}{|c|c|c|}
\hline Interventions & \multicolumn{2}{|c|}{$\begin{array}{l}\text { LABA + ICS vs SAME dose of ICS } \\
\text { OUTCOMES: Reported at 4, } 8 \text { and } 12 \text { weeks } \\
\text { RUN IN PERIOD: } 2 \text { weeks } \\
\text { DOSE OF ICS DURING RUN-IN: Continued on usual ICS of at least } 400 \mathrm{mcg} / \mathrm{day} \\
\text { BDP } \\
\text { DOSE OPTIMISATION PERIOD: None } \\
\text { INTERVENTION PERIOD: } 12 \text { weeks } \\
\text { TEST GROUP: (Salm } 50+\text { ICS) Salmeterol } 50 \mathrm{mg} \text { bid + ICS } 400-2400 \mathrm{mg} / \mathrm{day} \\
\text { (average: } 750 \text { mcg/day) } \\
\text { CONTROL GROUP: (Placebo+ICS) Placebo + ICS } 400-2400 \mathrm{mg} / \text { day (average } 750 \\
\text { mcg/day) } \\
\text { DEVICE: Diskhaler } \\
\text { NUMBER OF DEVICES: } 2 \\
\text { COMPLIANCE: Evaluated using patient kept record booklets } \\
\text { CO-TREATMENT: Salbutamol as needed and any other prophylactic asthma } \\
\text { medication via diskhaler }\end{array}$} \\
\hline Outcomes & \multicolumn{2}{|c|}{$\begin{array}{l}\text { PULMONARY FUNCTION TEST: am PEF percent predicted*; pm PEF percent } \\
\text { predicted } \\
\text { SYMTPOM SCORES: Symptoms were recorded daily as either being present or } \\
\text { absent, wheeze or cough during day or night } \\
\text { FUNCTIONAL STATUS: Proportion symptom-free days; proportion symptom-free } \\
\text { nights; rescue medication use } \\
\text { INFLAMMATORY MARKERS: Not described } \\
\text { ADVERSE EFFECTS: Described } \\
\text { WITHDRAWALS: Described } \\
\text { primary outcome measure* }\end{array}$} \\
\hline Notes & \multicolumn{2}{|c|}{$\begin{array}{l}\text { Full-text publication } \\
\text { Funded by Allen \& Hanburys } \\
\text { Confirmation of methodology and data obtained. } \\
\text { User-defined number: } 750 \text { ( } 750 \mathrm{mcg} / \text { day })\end{array}$} \\
\hline \multicolumn{3}{|l|}{ Risk of bias } \\
\hline Item & Authors' judgement & Description \\
\hline Adequate sequence generation? & Yes & Computer generated random numbers \\
\hline Allocation concealment? & Yes & $\begin{array}{l}\text { Numbered coded envelopes supplied by } \\
\text { Pharmacy }\end{array}$ \\
\hline $\begin{array}{l}\text { Blinding? } \\
\text { All outcomes }\end{array}$ & Yes & Double-blind; identical placebo \\
\hline $\begin{array}{l}\text { Incomplete outcome data } \\
\text { addressed? } \\
\text { All outcomes }\end{array}$ & No & $\begin{array}{l}\text { 'Total population used, this comprised all } \\
\text { subjects who received at least one puff of } \\
\text { medication and recorded at least one day of } \\
\text { valid diary or clinic data during the treatment } \\
\text { period. Where a subject withdrew before } \\
\text { completion of the study, data recorded after } \\
\text { this withdrawal data was excluded.' }\end{array}$ \\
\hline Free of selective reporting? & Yes & OCS-treated exacerbation data available \\
\hline Free of other bias? & Unclear & $\begin{array}{l}\text { Information on } \% \text { screening population } \\
\text { eligible not available }\end{array}$ \\
\hline
\end{tabular}

SAM104926

\begin{tabular}{ll}
\hline Methods & Parallel group, multicentre study \\
\hline Participants & \% ELIGIBLE OF SCREENED POPULATION: Not reported \\
& \% RUN-IN PARTICIPANTS RANDOMISED: Not reported \\
& RANDOMISED: 321 (FP/SAL: 160; FP: 161) \\
WITHDRAWALS: FP/SAL: 3; FP: 6 \\
AGE mean (range) or mean (SD): $8(2)$
\end{tabular}

Cochrane Database Syst Rev. Author manuscript; available in PMC 2014 September 19. 
SEVERITY: Not reported

BASELINE \% PRED. FEV1: 102

BASELINE DOSE OF ICS: Not stated

ASTHMA DURATION: Not stated

ATOPY (\%): Not stated

ELIGIBILITY CRITERIA: 4-11 years; diagnosis of asthma for a minimum of 6

months; airway reversibility of $=15 \%$ based either on FEV1 or PEF; treatment with

medium dose ICS (beclomethasone dipropionate (BDP) equivalent 400-500

$\mathrm{mcg} /$ day for 3 months prior to Visit 1

EXCLUSION CRITERIA: Respiratory tract infection in previous 4 weeks; acute asthma exacerbation requiring emergency room treatment within the last 4 weeks/

hospitalisation within last 12 weeks; use of systemic corticosteroid within the last

12 weeks, or use of LABA, oral B2-agonists, leukotriene antagonists or

theophyllines during 4 weeks prior to screening visit; $\mathrm{e}$ for randomisation if, during

the run-in period, change in asthma medication including use of systemic

corticosteroids, or respiratory tract infection or asthma exacerbation

ELIGIBILITY CRITERIA DURING RUN IN: Asthma assessed as not 'Well

Controlled' for at least 2 of 4 weeks of run-in; FEV1 $>60 \%$ during run-in

\begin{tabular}{|c|c|c|}
\hline Interventions & \multicolumn{2}{|c|}{$\begin{array}{l}\text { ICS and LABA versus INCREASED dose ICS } \\
\text { OUTCOMES: } 12 \text { weeks } \\
\text { RUN IN PERIOD: } 4 \text { weeks } \\
\text { DOSE OPTIMISATION PERIOD: NA } \\
\text { INTERVENTION PERIOD: } 12 \text { weeks } \\
\text { TEST GROUP: Combination fluticasone and salmeterol 100/50 mcg BID, via DPI } \\
\text { CONTROL GROUP: Fluticasone } 200 \mathrm{mcg} \text { BID, via DPI } \\
\text { NUMBER OF DEVICES: } 1 \\
\text { COMPLIANCE: Not assessed } \\
\text { CO-TREATMENT: prn SABA }\end{array}$} \\
\hline Outcomes & \multicolumn{2}{|c|}{$\begin{array}{l}\text { PULMONARY FUNCTION TEST: Am PEF } \\
\text { SYMPTOM SCORES: NA } \\
\text { FUNCTIONAL STATUS: N achieving well controlled asthma } \\
\text { INFLAMMATORY MARKERS: NA } \\
\text { ADVERSE EFFECTS: Reported } \\
\text { WITHDRAWALS: Reported }\end{array}$} \\
\hline Notes & \multicolumn{2}{|c|}{$\begin{array}{l}\text { Unpublished study } \\
\text { Funding source: GSK } \\
\text { Confirmation of methodology and data not obtained. } \\
\text { User defined: } 800\end{array}$} \\
\hline \multicolumn{3}{|l|}{ Risk of bias } \\
\hline Item & Authors' judgement & Description \\
\hline Adequate sequence generation? & Yes & See Appendix 1 \\
\hline Allocation concealment? & Yes & See Appendix 1 \\
\hline $\begin{array}{l}\text { Blinding? } \\
\text { All outcomes }\end{array}$ & Yes & Double-blind; double dummy \\
\hline $\begin{array}{l}\text { Incomplete outcome data } \\
\text { addressed? } \\
\text { All outcomes }\end{array}$ & Unclear & $\begin{array}{l}\text { No detailed information on how intention to } \\
\text { treat population was composed }\end{array}$ \\
\hline Free of selective reporting? & Unclear & $\begin{array}{l}\text { Unclear whether data on OCS-treated } \\
\text { exacerbations were collected. Request for } \\
\text { data from study sponsors has not been } \\
\text { successful. Data on hospital admission } \\
\text { available from published meta-analysis }\end{array}$ \\
\hline Free of other bias? & Unclear & $\begin{array}{l}\text { Information on } \% \text { screening population } \\
\text { eligible not reported }\end{array}$ \\
\hline
\end{tabular}

SAM40012a

\begin{tabular}{ll}
\hline Methods & Parallel group, multicentre study in Europe and Middle East. \\
\hline Participants & Steroid-using asthmatic children \\
\%ELIGIBLE OF SCREENED POPULATION: Not reported
\end{tabular}

Cochrane Database Syst Rev. Author manuscript; available in PMC 2014 September 19. 
\%RUN-IN PARTICIPANTS RANDOMISED: Not reported

RANDOMISED: 362 (FP/SAL: 181; FP: 181)

WITHDRAWAL: FP/SAL: 3 ; FP: 10

AGE mean: 8 years

GENDER (male\%): 68

ASTHMA SEVERITY: Moderate

BASELINE \% PRED. FEV1 mean: Not reported

BASELINE DOSE OF ICS (start of run in): Not reported

ASTHMA DURATION: Not reported

ATOPY(\%): Not reported

ELIGIBILITY CRITERIA: 4-500mcg BDP equivalent; documented history of

asthma

EXCLUSION CRITERIA: Not reported

ELIGIBILITY CRITERIA DURING RUN-IN: Symptom score $>/=2$ on three of last seven days of run-in

Interventions

LABA + ICS versus SAME dose of ICS

OUTCOMES: Reported at 6 months

RUN IN PERIOD: 2 weeks

DOSE OF ICS DURING RUN IN: Not clear

DOSE OPTIMISATION PERIOD: None reported

INTERVENTION PERIOD: 6 months

TEST GROUP: Combination Salmeterol 50/Fluticasone 100mcg bid

CONTROL GROUP: Fluticasone 100mcg bid

DEVICE: Diskus

NUMBER OF DEVICES: 1

COMPLIANCE: Not reported

CO-TREATMENT: prn SABA

\begin{tabular}{|c|c|c|}
\hline Outcomes & \multicolumn{2}{|c|}{$\begin{array}{l}\text { OUTCOMES: Reported at } 6 \text { months } \\
\text { PULMONARY FUNCTION TEST: am PEF; pm PEF; FEV1 } \\
\text { SYMPTOM SCORES: Symptom-free days } \\
\text { FUNCTIONAL STATUS: Use of reliever medication; exacerbations (undefined) } \\
\text { INFLAMMATORY MARKERS: Not reported } \\
\text { ADVERSE EFFECTS: Reported } \\
\text { WITHDRAWAL: Reported } \\
\text { *primary outcome }\end{array}$} \\
\hline Notes & \multicolumn{2}{|c|}{$\begin{array}{l}\text { Full unpublished data set available from http://www.ctr.gsk.co.uk } \\
\text { Source of funding: GSK } \\
\text { Confirmation of methodology and data: Not obtained } \\
\text { User defined number: } 400\end{array}$} \\
\hline \multicolumn{3}{|l|}{ Risk of bias } \\
\hline Item & Authors' judgement & Description \\
\hline Adequate sequence generation? & Yes & See Appendix 1 \\
\hline Allocation concealment? & Yes & See Appendix 1 \\
\hline $\begin{array}{l}\text { Blinding? } \\
\text { All outcomes }\end{array}$ & Yes & Identical inhaler devices \\
\hline $\begin{array}{l}\text { Incomplete outcome data } \\
\text { addressed? } \\
\text { All outcomes }\end{array}$ & Unclear & $\begin{array}{l}\text { 'To be evaluable, subjects had to meet the entry and } \\
\text { randomisation criteria, receive at least one dose of study } \\
\text { medication and have completed at least one day's post- } \\
\text { randomisation diary information.' }\end{array}$ \\
\hline Free of selective reporting? & Unclear & $\begin{array}{l}\text { Exacerbations described in trial report available; OCS- } \\
\text { treated exacerbations could not be identified from the } \\
\text { data available }\end{array}$ \\
\hline Free of other bias? & Unclear & Information not available \\
\hline
\end{tabular}

SAM40012b

\begin{tabular}{ll}
\hline Methods & See SAM40012a \\
\hline Participants & See SAM40012a, except for \\
& RANDOMISED: FP/SAL: 181; FP: 186 \\
& WITHDRAWAL: FP/SAL: 3; FP: 5 \\
\hline
\end{tabular}

Cochrane Database Syst Rev. Author manuscript; available in PMC 2014 September 19. 


\begin{tabular}{lll} 
Interventions & See SAM40012a & \\
\hline Outcomes & See SAM40012a & \\
\hline Notes & See SAM40012a & \\
\hline Risk of bias & & \\
\hline Item & Authors' judgement & Description \\
\hline Adequate sequence generation? & Yes & See Appendix 1 \\
\hline Allocation concealment? & Yes & See Appendix 1 \\
\hline $\begin{array}{l}\text { Blinding? } \\
\text { All outcomes }\end{array}$ & Yes & See SAM40012a \\
\hline $\begin{array}{l}\text { Incomplete outcome data addressed? } \\
\text { All outcomes }\end{array}$ & Unclear & See SAM40012a \\
\hline Free of selective reporting? & Unclear & See SAM40012a \\
\hline Free of other bias? & Unclear & See SAM40012a \\
\hline
\end{tabular}

SAM40100

\begin{tabular}{|c|c|}
\hline Methods & Parallel group, multicentre study \\
\hline Participants & $\begin{array}{l}\text { \% ELIGIBLE OF SCREENED POPULATION: Not reported } \\
\% \text { RUN-IN PARTICIPANTS RANDOMISED: Not reported } \\
\text { RANDOMISED: } 24 \text { (FP/SAL: } 12 \text {; FP: 12) } \\
\text { WITHDRAWALS: F/SAL: 1; FP: } 1 \\
\text { AGE mean: } 7.3 \\
\text { SEVERITY: Not stated. } \\
\text { BASELINE \% PRED. FEV1: Not reported. } \\
\text { BASELINE DOSE OF ICS: Not stated. } \\
\text { ASTHMA DURATION: Not reported } \\
\text { ATOPY (\%): Not reported } \\
\text { ELIGIBILITY CRITERIA: } 4-8 \text { years; history of asthma for at least } 3 \text { months; } \\
\text { maintenance ICS dose of 200-800mcg/day BDP or equivalent for at least } 4 \text { weeks; } \\
\text { sufficiently stable to receive FP 200mcg/day during 2-week run-in; sRAW value of } \\
\text { =1.3kPa.s for entry into the screening and treatment period } \\
\text { EXCLUSION CRITERIA: Use of systemic steroids in } 4 \text { weeks prior to study } \\
\text { entry; required } 3 \text { or more courses of oral corticosteroids in } 12 \text { months prior to study } \\
\text { entry; admitted to intensive care for asthma within } 3 \text { months prior to study entry } \\
\text { ELIGIBILITY CRITERIA DURING RUN IN: Participants who had a change in } \\
\text { medication following an exacerbation during run-in were excluded }\end{array}$ \\
\hline Interventions & $\begin{array}{l}\text { LABA+ICS versus INCREASED DOSE ICS } \\
\text { OUTCOMES: } 6 \text { weeks } \\
\text { RUN IN PERIOD: } 2 \text { weeks } \\
\text { DOSE OPTIMISATION PERIOD: } 2 \text { weeks } \\
\text { INTERVENTION PERIOD: } 6 \text { weeks } \\
\text { TEST GROUP: Combination fluticasone and salmeterol 100/50mcg BID via DPI } \\
\text { CONTROL GROUP: Fluticasone 200mcg BID via DPI } \\
\text { NUMBER OF DEVICES: } 1 \\
\text { COMPLIANCE: Not assessed } \\
\text { CO-TREATMENT: prn SABA }\end{array}$ \\
\hline Outcomes & $\begin{array}{l}\text { PULMONARY FUNCTION TEST: FEV1 } \\
\text { SYMPTOM SCORES: Day and nocturnal scores } \\
\text { FUNCTIONAL STATUS: Rescue medication use } \\
\text { INFLAMMATORY MARKERS: sRAW* } \\
\text { ADVERSE EFFECTS: Reported } \\
\text { WITHDRAWALS: Reported }\end{array}$ \\
\hline Notes & $\begin{array}{l}\text { Unpublished data sourced from http://ctr.gsk.co.uk } \\
\text { Funding source: GSK } \\
\text { Confirmation of methodology and data not obtained. } \\
\text { User defined: } 400\end{array}$ \\
\hline Risk of bias & \\
\hline
\end{tabular}




\begin{tabular}{|c|c|c|}
\hline Item & Authors' judgement & Description \\
\hline Adequate sequence generation? & Yes & See Appendix 1 \\
\hline Allocation concealment? & Yes & See Appendix 1 \\
\hline $\begin{array}{l}\text { Blinding? } \\
\text { All outcomes }\end{array}$ & Yes & Double-blind; identical devices used \\
\hline $\begin{array}{l}\text { Incomplete outcome data } \\
\text { addressed? } \\
\text { All outcomes }\end{array}$ & Unclear & $\begin{array}{l}\text { No detailed information on how } \\
\text { intention to treat population was } \\
\text { composed }\end{array}$ \\
\hline Free of selective reporting? & Unclear & $\begin{array}{l}\text { Unclear whether data on OCS-treated } \\
\text { exacerbations were collected. Reques } \\
\text { for data from study sponsors has not } \\
\text { been successful }\end{array}$ \\
\hline Free of other bias? & Unclear & $\begin{array}{l}\text { Information on } \% \text { screening/run-in } \\
\text { population eligible not reported }\end{array}$ \\
\hline
\end{tabular}

SD 0390714

\begin{tabular}{|c|c|}
\hline Methods & Parallel group, multicentre study \\
\hline Participants & $\begin{array}{l}\text { Steroid-using symptomatic asthmatic adolescents } \\
\text { \%ELIGIBLE OF SCREENED POPULATION Not reported } \\
\text { \%RUN-IN PARTICIPANTS RANDOMISED: 60\% } \\
\text { RANDOMISED: } 271 \text { (F6/Bud 200mcg bid: 136; Bud 200mcg bid: 135) } \\
\text { WITHDRAWAL: F6/Bud 200mcg bid: 25; Bud 200mcg bid: } 27 \\
\text { AGE: mean (range): } 14 \text { (11-17) } \\
\text { GENDER (male\%): } 42 \\
\text { ASTHMA SEVERITY: Moderate } \\
\text { BASELINE \% PRED. FEV1 mean: } 75 \\
\text { BASELINE DOSE OF ICS (start of run in): Not reported } \\
\text { ASTHMA DURATION: Not reported } \\
\text { ATOPY(\%): Not reported } \\
\text { ELIGIBILITY CRITERIA: ICS 375-1000mcg BDP equivalent; FEV1 40-90\% } \\
\text { predicted normal; >= 12\% improvement following inhalation of 1mg of terbutaline } \\
\text { EXCLUSION CRITERIA: Not reported } \\
\text { CRITERIA FOR RANDOMISATION DURING RUN-IN: Symptomatic }\end{array}$ \\
\hline Interventions & $\begin{array}{l}\text { LABA + ICS versus SAME dose of ICS } \\
\text { OUTCOMES: Reported at } 1,2 \text { and } 3 \text { months } \\
\text { RUN IN PERIOD: } 2 \text { weeks to document stability } \\
\text { DOSE OF ICS DURING RUN IN: Not clear } \\
\text { DOSE OPTIMISATION PERIOD: None reported } \\
\text { INTERVENTION PERIOD: } 3 \text { months } \\
\text { TEST GROUP: Combination budesonide and formoterol } 200 / 6 \mathrm{mcg} \text { bid } \\
\text { CONTROL GROUP: Budesonide } 200 \mathrm{mcg} \text { bid } \\
\text { DEVICE:-Turbuhaler } \\
\text { NUMBER OF DEVICES: } 1 \\
\text { COMPLIANCE: Not reported } \\
\text { CO-TREATMENT: prn SABA }\end{array}$ \\
\hline Outcomes & $\begin{array}{l}\text { PULMONARY FUNCTION TEST: FEV1; am PEF*; pm PEF } \\
\text { SYMPTOM SCORES: Recorded but not reported } \\
\text { FUNCTIONAL STATUS: Rescue medication use (recorded but not reported); } \\
\text { nocturnal awakening (recorded but not reported); episode free days (recorded but } \\
\text { not reported) } \\
\text { INFLAMMATORY MARKERS: Not reported } \\
\text { ADVERSE EFFECTS: Reported } \\
\text { WITHDRAWAL: Reported } \\
\text { * primary outcome }\end{array}$ \\
\hline Notes & $\begin{array}{l}\text { Unpublished data downloaded from AZ website } \\
\text { (www.astrazenecaclinicaltrials.com) } \\
\text { Funded by AstraZeneca } \\
\text { Confirmation of data and methodology: Obtained } \\
\text { User defined: } 400\end{array}$ \\
\hline
\end{tabular}




\begin{tabular}{lll} 
Risk of bias & & \\
\hline Item & Authors' judgement & Description \\
\hline Adequate sequence generation? & Unclear & Information not available \\
\hline Allocation concealment? & Unclear & Information not available \\
\hline $\begin{array}{l}\text { Blinding? } \\
\text { All outcomes }\end{array}$ & Yes & Double-blind; double dummy \\
\hline $\begin{array}{l}\text { Incomplete outcome data } \\
\text { addressed? }\end{array}$ & Unclear & $\begin{array}{l}\text { No detailed information on how intention to treat } \\
\text { population was composed }\end{array}$ \\
\hline Free of selective reporting? & Unclear & $\begin{array}{l}\text { OCS-treated exacerbations were not reported in the } \\
\text { study publication. Data request has been made to } \\
\text { study sponsors for this information }\end{array}$ \\
\hline Free of other bias? & & \begin{tabular}{l} 
59\% run-in population eligible \\
\hline
\end{tabular} \\
\hline
\end{tabular}

SD 0390718

\begin{tabular}{|c|c|}
\hline Methods & Parallel group; multicentre study ( 52 centres in USA) \\
\hline Participants & $\begin{array}{l}\% \text { ELIGIBLE OF SCREENED POPULATION: Not reported } \\
\% \text { RUN-IN PARTICIPANTS RANDOMISED } 60 \\
\text { RANDOMISED: } 273 \text { (BUD/F: 128; BUD: } 145 \text { ) } \\
\text { WITHDRAWALS: BUD/F: 36; BUD: } 51 \\
\text { AGE mean (range) or mean (SD): } 10.4 \text { (2.6) } \\
\text { SEVERITY: Not stated } \\
\text { BASELINE \% PRED. FEV1: } 82 \\
\text { BASELINE DOSE OF ICS: } 235 \mathrm{mcg} / \mathrm{d} \\
\text { ASTHMA DURATION: } 7 \text { years } \\
\text { ATOPY (\%): Not stated } \\
\text { ELIGIBILITY CRITERIA: } 6-15 \text { years; low to medium dose of ICS; FEV1 } \\
\text { predicted > 50\%; reversibility criteria age dependent: >12 years } 14 \% \text { and } 0.2 \mathrm{~L} ;<12 \\
\text { years: } 12 \% \\
\text { EXCLUSION CRITERIA: Not reported. } \\
\text { ELIGIBILITY CRITERIA DURING RUN IN: Symptoms and lung function not } \\
\text { otherwise described }\end{array}$ \\
\hline Interventions & $\begin{array}{l}\text { LABA+ICS versus SAME DOSE ICS } \\
\text { OUTCOMES: } 12 \text { weeks } \\
\text { RUN IN PERIOD: } 1-2 \text { weeks } \\
\text { DOSE OPTIMISATION PERIOD: Not applicable } \\
\text { INTERVENTION PERIOD: } 12 \text { weeks } \\
\text { TEST GROUP: Combination budesonide/formoterol }(100 / 9 \mathrm{mcg}) \text { BID via metered } \\
\text { dose inhaler } \\
\text { CONTROL GROUP: Budesonide } 100 \mathrm{mcg} \text { BID via metered dose inhaler } \\
\text { NUMBER OF DEVICES: } 1 \\
\text { COMPLIANCE: Not assessed } \\
\text { CO-TREATMENT: prn SABA }\end{array}$ \\
\hline Outcomes & $\begin{array}{l}\text { PULMONARY FUNCTION TEST: Am PEF; pm PEF; FEV1 } \\
\text { SYMPTOM SCORES: NA } \\
\text { FUNCTIONAL STATUS: NA } \\
\text { INFLAMMATORY MARKERS: NA } \\
\text { ADVERSE EFFECTS: Stated } \\
\text { WITHDRAWALS: } \text { Stated }\end{array}$ \\
\hline Notes & $\begin{array}{l}\text { Unpublished data from AZ clinical trials website } \\
\text { Funded by AstraZeneca } \\
\text { Confirmation of data and methodology: Obtained } \\
\text { User defined: } 200\end{array}$ \\
\hline \multicolumn{2}{|l|}{ Risk of bias } \\
\hline Item & Authors' judgement Description \\
\hline Adequate sequence generation? & Described as randomised; no other information presented \\
\hline
\end{tabular}




\begin{tabular}{lll} 
Allocation concealment? & Unclear & Information not available \\
\hline $\begin{array}{l}\text { Blinding? } \\
\text { All outcomes }\end{array}$ & Yes & Double-blind; double dummy \\
\hline $\begin{array}{l}\text { Incomplete outcome data } \\
\text { addressed? } \\
\text { All outcomes }\end{array}$ & Unclear & $\begin{array}{l}\text { 'The efficacy analysis set (EAS) was defined as all } \\
\text { randomized subjects who took at least 1 dose of study } \\
\text { medication and contributed at least 1 PEF value to the } \\
\text { primary end-point.' } \\
\text { No information given on whether EAS population } \\
\text { included last observation }\end{array}$ \\
\hline Free of selective reporting? & Unclear & $\begin{array}{l}\text { OCS-treated exacerbations were not reported in the study } \\
\text { publication. Data request has been made to study } \\
\text { sponsors for this information }\end{array}$ \\
\hline Free of other bias? & Yes & 59\% screening population eligible \\
\hline
\end{tabular}

SD 0390719

\begin{tabular}{|c|c|}
\hline Methods & Parallel group, multicentre study. \\
\hline Participants & $\begin{array}{l}\% \text { ELIGIBLE OF SCREENED POPULATION: Not stated } \\
\% \text { RUN-IN PARTICIPANTS RANDOMISED: } 74 \\
\text { RANDOMISED: } 186 \text { (BUD/F: 123; BUD: 63) } \\
\text { WITHDRAWALS: BUD/F: 13; BUD: } 10 \\
\text { AGE mean (range) or mean (SD): } 9 \text { (1.7) } \\
\text { SEVERITY: Not stated } \\
\text { BASELINE \% PRED. FEV1: } 84 \% \\
\text { BASELINE DOSE OF ICS: } 307 \\
\text { ASTHMA DURATION: } 6 \text { years } \\
\text { ATOPY (\%): Not stated } \\
\text { ELIGIBILITY CRITERIA: 6-12 years; inhaled steroid-dependent asthma; FEV1 } \\
\text { >50\% of predicted; history of PEF or FEV1 reversibility } 12 \% \text {; subjects without } \\
\text { history of reversibility must have demonstrated FEV1 reversibility as above at any } \\
\text { time before Visit } 2 \\
\text { EXCLUSION CRITERIA: Not reported } \\
\text { ELIGIBILITY CRITERIA DURING RUN IN: Not reported }\end{array}$ \\
\hline Interventions & $\begin{array}{l}\text { LABA and ICS versus SAME dose ICS } \\
\text { OUTCOMES: } 26 \text { weeks } \\
\text { RUN IN PERIOD: } 1 \text { week } \\
\text { DOSE OPTIMISATION PERIOD: NA } \\
\text { INTERVENTION PERIOD: } 26 \text { weeks } \\
\text { TEST GROUP: Combination budesonide and formoterol 160/4.5 mcg per } \\
\text { actuation, } 2 \text { puffs BID via MDI } \\
\text { CONTROL GROUP: Budesonide } 160 \mathrm{mcg} \text { per actuation, } 2 \text { puffs BID via MDI } \\
\text { NUMBER OF DEVICES: } 1 \\
\text { COMPLIANCE: Not assessed } \\
\text { CO-TREATMENT: prn SABA }\end{array}$ \\
\hline Outcomes & $\begin{array}{l}\text { PULMONARY FUNCTION TEST: FEV1; am PEF } \\
\text { SYMPTOM SCORES: NA } \\
\text { FUNCTIONAL STATUS: Paediatric AQLQ } \\
\text { INFLAMMATORY MARKERS: NA } \\
\text { ADVERSE EFFECTS: Adverse events* } \\
\text { WITHDRAWALS: Stated per treatment group } \\
\text { *Primary outcome }\end{array}$ \\
\hline Notes & $\begin{array}{l}\text { Downloaded from AZ clinical trials website (accessed } 4^{\text {th }} \text { January 2008) } \\
\text { Funded by Astra Zeneca } \\
\text { Confirmation of data: Provided by AZ in April } 2008 \\
\text { User-defined number: } 640\end{array}$ \\
\hline \multicolumn{2}{|l|}{ Risk of bias } \\
\hline Item & Authors' judgement \\
\hline Adequate sequence generation? & $\begin{array}{l}\text { Described as randomised; no other } \\
\text { information presented }\end{array}$ \\
\hline
\end{tabular}




\begin{tabular}{|c|c|c|}
\hline Allocation concealment? & Unclear & Information not available \\
\hline $\begin{array}{l}\text { Blinding? } \\
\text { All outcomes }\end{array}$ & No & Open label \\
\hline $\begin{array}{l}\text { Incomplete outcome data } \\
\text { addressed? } \\
\text { All outcomes }\end{array}$ & Unclear & $\begin{array}{l}\text { 'The safety analysis set was defined as all } \\
\text { randomized subjects who took at least } 1 \\
\text { dose of study medication.' } \\
\text { No information given on whether safety } \\
\text { population included last observation }\end{array}$ \\
\hline Free of selective reporting? & No & $\begin{array}{l}\text { OCS-treated exacerbations were not } \\
\text { reported in the study publication. Data } \\
\text { request has been made to study sponsors for } \\
\text { this information }\end{array}$ \\
\hline Free of other bias? & Yes & $\begin{array}{l}74 \% \text { screening population eligible for } \\
\text { randomisation }\end{array}$ \\
\hline
\end{tabular}

SD 039 0725a

\begin{tabular}{|c|c|}
\hline Methods & Parallel group, multicentre study \\
\hline Participants & $\begin{array}{l}\text { \% ELIGIBLE OF SCREENED POPULATION: } 35 \\
\% \text { RUN-IN PARTICIPANTS RANDOMISED: } 79 \\
\text { RANDOMISED: } 521 \text { (BUD/F BID: 184; BUD/F QD: 168; BUD: 169) } \\
\text { WITHDRAWALS: BUD/F BID: } 21 \text {; BUD/F QD: 37; BUD: } 33 \\
\text { AGE mean (range) or mean (SD): } 10.3 \text { (2.5) } \\
\text { SEVERITY: Not stated } \\
\text { BASELINE \% PRED. FEV1: } 78.3(8.56) \\
\text { BASELINE DOSE OF ICS: } 245.3 \\
\text { ASTHMA DURATION: } 6.8 \\
\text { ATOPY (\%): Not reported } \\
\text { ELIGIBILITY CRITERIA: } 6-15 \text { years; diagnosis of asthma for at least } 6 \text { months; } \\
\text { maintenance inhaled corticosteroids treatment for at least } 4 \text { weeks prior to } \\
\text { screening; FEV1 predicted } 60-90 \% \text { predicted; reversibility of FEV1 of } 12 \% \text { or } \\
\text { more and >0.20 L from baseline; children >11 years were required to demonstrate } \\
\text { reversibility of >12\% only } \\
\text { EXCLUSION CRITERIA: Not stated. } \\
\text { ELIGIBILITY CRITERIA DURING RUN IN Stable asthma symptoms }\end{array}$ \\
\hline Interventions & $\begin{array}{l}\text { LABA and ICS versus SAME DOSE ICS } \\
\text { OUTCOMES: } 12 \text { weeks } \\
\text { RUN IN PERIOD: } 4-5 \text { weeks } \\
\text { DOSE OPTIMISATION PERIOD: Not reported } \\
\text { INTERVENTION PERIOD: } 12 \text { weeks } \\
\text { TEST GROUP: Combination budesonide and formoterol 80/9mcg BID via MDI } \\
\text { CONTROL GROUP: Budesonide } 160 \mathrm{mcg} \text { QD via MDI } \\
\text { NUMBER OF DEVICES: } 1 \\
\text { COMPLIANCE: Not assessed } \\
\text { CO-TREATMENT: prn SABA }\end{array}$ \\
\hline Outcomes & $\begin{array}{l}\text { PULMONARY FUNCTION TEST: FEV1; Am PEF; Pm PEF** } \\
\text { SYMPTOM SCORES: Day \& nocturnal symptoms } \\
\text { FUNCTIONAL STATUS: AQLQ } \\
\text { INFLAMMATORY MARKERS: Not reported } \\
\text { ADVERSE EFFECTS: Reported } \\
\text { WITHDRAWALS: Reported by treatment group }\end{array}$ \\
\hline Notes & $\begin{array}{l}\text { Funding source: AZ } \\
\text { Confirmation of methodology and data obtained from AZ in April } 2008 \\
\text { Unpublished data downloaded from: http://www.astrazenecaclinicaltrials.com } \\
\text { User defined: } 160\end{array}$ \\
\hline \multicolumn{2}{|l|}{ Risk of bias } \\
\hline Item & Authors' judgement \\
\hline Adequate sequence generation? & $\begin{array}{l}\text { Described as randomised; no other information } \\
\text { presented. Age-based strata to ensure balance in ages } \\
\text { between groups ( } 6-11 \text { years \& } 12-15 \text { years) }\end{array}$ \\
\hline
\end{tabular}




\begin{tabular}{lll} 
Allocation concealment? & Unclear & Information not available \\
\hline $\begin{array}{l}\text { Blinding? } \\
\text { All outcomes }\end{array}$ & Yes & Double-blind; double dummy \\
\hline $\begin{array}{l}\text { Incomplete outcome data } \\
\text { addressed? } \\
\text { All outcomes }\end{array}$ & Unclear & $\begin{array}{l}\text { Efficacy analysis does not explicitly describe } \\
\text { whether missing data imputed or drawn from follow- } \\
\text { up: 'all randomized subjects who took at least 1 dose } \\
\text { of double-blind treatment, and who contributed at } \\
\text { least 1 evening PEF diary entry after receiving } \\
\text { double-blind medication, was used in the primary } \\
\text { analysis.' }\end{array}$ \\
\hline Free of selective reporting? & No & $\begin{array}{l}\text { OCS-treated exacerbations were not reported in the } \\
\text { study publication. Data request has been made to } \\
\text { study sponsors for this information }\end{array}$ \\
\hline Free of other bias? & Yes & 37\% screening population eligible for randomisation \\
\hline
\end{tabular}

SD 039 0725b

\begin{tabular}{|c|c|c|}
\hline Methods & \multicolumn{2}{|l|}{ See SD 039 0725b } \\
\hline Participants & \multicolumn{2}{|l|}{ See SD 039 0725b } \\
\hline Interventions & \multicolumn{2}{|c|}{$\begin{array}{l}\text { See SD } 0390725 \mathrm{~b} \\
\text { TEST GROUP: } \\
\text { Combination budesonide and formoterol 160/9mcg QD via MDI }\end{array}$} \\
\hline Outcomes & \multicolumn{2}{|l|}{ See SD 039 0725b } \\
\hline Notes & \multicolumn{2}{|l|}{ See SD 039 0725b } \\
\hline \multicolumn{3}{|l|}{ Risk of bias } \\
\hline Item & Authors' judgement & Description \\
\hline Adequate sequence generation? & Unclear & Information not available \\
\hline Allocation concealment? & Unclear & Information not available \\
\hline $\begin{array}{l}\text { Blinding? } \\
\text { All outcomes }\end{array}$ & Yes & Double-blind; double dummy \\
\hline $\begin{array}{l}\text { Incomplete outcome data addressed? } \\
\text { All outcomes }\end{array}$ & Unclear & $\begin{array}{l}\text { Efficacy analysis does not explicitly describe whether } \\
\text { missing data imputed or drawn from follow-up: } \\
\text { 'all randomized subjects who took at least } 1 \text { dose of } \\
\text { double-blind treatment, and who contributed at least } 1 \\
\text { evening PEF diary entry after receiving double-blind } \\
\text { medication, were used in the primary analysis.' }\end{array}$ \\
\hline Free of selective reporting? & No & $\begin{array}{l}\text { OCS-treated exacerbations were not reported in the } \\
\text { study publication. Data request has been made to study } \\
\text { sponsors for this information }\end{array}$ \\
\hline Free of other bias? & Yes & $37 \%$ screening population eligible for randomisation \\
\hline
\end{tabular}

\section{SFA100314}

\begin{tabular}{ll}
\hline Methods & Parallel group, multicentre study (51 centres in USA) \\
\hline Participants & \% ELIGIBLE OF SCREENED POPULATION: Not reported \\
& \% RUN-IN PARTICIPANTS RANDOMISED: Not reported \\
& RANDOMISED: 248 (FP/SAL: $124 ;$ FP: 124) \\
WITHDRAWALS: FP/SAL: $13 / 124 ;$ FP: $22 / 124$ \\
AGE mean (range) or mean (SD): 11 \\
SEVERITY: Not reported
\end{tabular}

Cochrane Database Syst Rev. Author manuscript; available in PMC 2014 September 19. 
BASELINE \% PRED. FEV1: Not reported

BASELINE DOSE OF ICS: $100 \mathrm{mcg} /$ day

ASTHMA DURATION: Not reported

ATOPY (\%): Not reported

ELIGIBILITY CRITERIA: Diagnosed with persistent asthma for 3 months or

longer; experience worsened asthma symptoms during physical activity; using or used an inhaled steroid for the last 4 weeks or longer (such as Aerobid, Azmacort,

Flovent, Pulmicort, QVAR, or Vanceril)

EXCLUSION CRITERIA: Use of systemic steroids as either liquids, pills, or injections to treat asthma within the last 3 months; intermittent, seasonal, or exercise induced asthma, and not persistent asthma; admitted to a hospital within the last 6 months due to asthma symptoms; poorly controlled medical conditions that may make study participation unsafe or inappropriate in the opinion of the study physician (such as cystic fibrosis, congenital heart disease, insulin dependent diabetes, glaucoma, drug allergies, etc.)

ELIGIBILITY CRITERIA DURING RUN IN : Not reported

\begin{tabular}{|c|c|c|}
\hline Interventions & \multicolumn{2}{|c|}{$\begin{array}{l}\text { PROTOCOL: LABA+ICS versus SAME dose ICS } \\
\text { OUTCOMES: } 4 \text { weeks } \\
\text { RUN IN PERIOD: } 7-14 \text { days } \\
\text { DOSE OPTIMISATION PERIOD: Not reported } \\
\text { INTERVENTION PERIOD: } 4 \text { weeks } \\
\text { TEST GROUP: Combination fluticasone and salmeterol 100/50mcg bid } \\
\text { CONTROL GROUP: Fluticasone 100mcg bid } \\
\text { NUMBER OF DEVICES: } 1 \\
\text { COMPLIANCE: Not assessed } \\
\text { CO-TREATMENT: prn SABA }\end{array}$} \\
\hline Outcomes & \multicolumn{2}{|c|}{$\begin{array}{l}\text { PULMONARY FUNCTION TEST: FEV1 AUC; } \\
\text { SYMPTOM SCORES: Not reported } \\
\text { FUNCTIONAL STATUS: exacerbations requiring rescue oral steroids } \\
\text { INFLAMMATORY MARKERS: Not reported } \\
\text { ADVERSE EFFECTS: Reported } \\
\text { WITHDRAWALS: Reported }\end{array}$} \\
\hline Notes & \multicolumn{2}{|c|}{$\begin{array}{l}\text { Funding source: GSK } \\
\text { Confirmation of methodology and data obtained from GSK in August } 2008 \\
\text { Unpublished data downloaded from: www.ctr.gsk.co.uk } \\
\text { User defined: } 400\end{array}$} \\
\hline \multicolumn{3}{|l|}{ Risk of bias } \\
\hline Item & Authors' judgement & Description \\
\hline Adequate sequence generation? & Yes & See Appendix 1 \\
\hline Allocation concealment? & Yes & See Appendix 1 \\
\hline $\begin{array}{l}\text { Blinding? } \\
\text { All outcomes }\end{array}$ & Yes & Identical in haler devices used \\
\hline $\begin{array}{l}\text { Incomplete outcome data } \\
\text { addressed? } \\
\text { All outcomes }\end{array}$ & Unclear & $\begin{array}{l}\text { The ITT population consisted of all } \\
\text { subjects who were randomized to } \\
\text { study drug.' }\end{array}$ \\
\hline Free of selective reporting? & Yes & $\begin{array}{l}\text { OCS-treated exacerbation data } \\
\text { available from GSK on request }\end{array}$ \\
\hline Free of other bias? & Unclear & $\begin{array}{l}\mathrm{N} \text { of screening population not } \\
\text { reported }\end{array}$ \\
\hline
\end{tabular}

SFA100316

\begin{tabular}{ll}
\hline Methods & Parallel group, multicentre study (49 centres in USA) \\
\hline Participants & \% ELIGIBLE OF SCREENED POPULATION: Not reported \\
& \% RUN-IN PARTICIPANTS RANDOMISED: Not reported \\
& RANDOMISED: 231 (FP/SAL: $113 ;$ FP: 118 ) \\
WITHDRAWALS: FP/SAL: 7/113; FP 10/118 \\
AGE mean (range) or mean (SD): 11.6 \\
SEVERITY: Not reported \\
BASELINE \% PRED. FEV1: Not reported
\end{tabular}

Cochrane Database Syst Rev. Author manuscript; available in PMC 2014 September 19. 
BASELINE DOSE OF ICS: FP 100mcg

ASTHMA DURATION: Not reported

ATOPY (\%): Not reported

ELIGIBILITY CRITERIA: Diagnosed with persistent asthma for 3 months or

longer; experience worsened asthma symptoms during physical activity; using or

used an inhaled steroid for the last 4 weeks or longer (such as Aerobid, Azmacort,

Flovent, Pulmicort, QVAR, or Vanceril)

EXCLUSION CRITERIA: Use of systemic steroids as either liquids, pills, or

injections to treat asthma within the last 3 months; intermittent, seasonal, or

exercise induced asthma, and not persistent asthma; admitted to a hospital within

the last 6 months due to asthma symptoms; poorly controlled medical conditions

that may make study participation unsafe or inappropriate in the opinion of the

study physician (such as cystic fibrosis, congenital heart disease, insulin dependent

diabetes, glaucoma, drug allergies, etc.)

ELIGIBILITY CRITERIA DURING RUN IN: Not reported

Interventions

PROTOCOL: LABA+ICS versus SAME dose ICS

OUTCOMES: 4 weeks

RUN IN PERIOD: 7-14 days

DOSE OPTIMISATION PERIOD: Not reported

INTERVENTION PERIOD: 4 weeks

TEST GROUP: Combination fluticasone and salmeterol 100/50mcg bid

CONTROL GROUP: Fluticasone 100mcg bid

NUMBER OF DEVICES: 1

COMPLIANCE: Not assessed

CO-TREATMENT: prn SABA

\begin{tabular}{|c|c|c|}
\hline Outcomes & \multicolumn{2}{|c|}{$\begin{array}{l}\text { PULMONARY FUNCTION TEST: FEV1 AUC. } \\
\text { SYMPTOM SCORES: Not reported } \\
\text { FUNCTIONAL STATUS: exacerbations requiring rescue oral steroids } \\
\text { INFLAMMATORY MARKERS: Not reported } \\
\text { ADVERSE EFFECTS: Reported } \\
\text { WITHDRAWALS: Reported }\end{array}$} \\
\hline Notes & \multicolumn{2}{|c|}{$\begin{array}{l}\text { Funding source: GSK } \\
\text { Confirmation of methodology and data obtained from GSK in August } 2008 \\
\text { Unpublished data downloaded from: www.ctr.gsk.co.uk } \\
\text { User defined: } 400\end{array}$} \\
\hline \multicolumn{3}{|l|}{ Risk of bias } \\
\hline Item & Authors' judgement & Description \\
\hline Adequate sequence generation? & Yes & See Appendix 1 \\
\hline Allocation concealment? & Yes & See Appendix 1 \\
\hline $\begin{array}{l}\text { Blinding? } \\
\text { All outcomes }\end{array}$ & Yes & Identical in haler devices used \\
\hline $\begin{array}{l}\text { Incomplete outcome data } \\
\text { addressed? } \\
\text { All outcomes }\end{array}$ & Unclear & $\begin{array}{l}\text { Intention to treat population; no } \\
\text { further details on analytical model } \\
\text { used }\end{array}$ \\
\hline Free of selective reporting? & Yes & $\begin{array}{l}\text { OCS-treated exacerbation data } \\
\text { available from GSK on request }\end{array}$ \\
\hline Free of other bias? & Unclear & $\begin{array}{l}\mathrm{N} \text { of screening population not } \\
\text { reported }\end{array}$ \\
\hline
\end{tabular}

Simons 1997

\begin{tabular}{ll}
\hline Methods & Crossover, single centre study \\
\hline Participants & Asymptomatic Children \\
& \%ELIGIBLE OF SCREENED POPULATION: Not reported \\
& \%RUN-IN PARTICIPANTS RANDOMISED: Not reported \\
RANDOMISED: 16 \\
WITHDRAWALS: $2(13 \%)$ \\
AGE mean (range): $13.1(12-16$ yrs) \\
GENDER (\%male): 44 \\
SEVERITY: Not described
\end{tabular}

Cochrane Database Syst Rev. Author manuscript; available in PMC 2014 September 19. 
BASELINE \% PRED. FEV1: 93.4

BASELINE DOSE OF ICS: $100-200 \mathrm{mcg}$ BDP BID

ASTHMA DURATION: $5.9+/-3.4 \mathrm{yrs}$

ATOPY (\%): 100

ELIGIBILITY CRITERIA: 12 to 18 years old; well-controlled chronic asthma; diagnosed according to American Thoracic Society criteria; able to perform treadmill running tests; do pulmonary function tests satisfactorily; use a Nebulizer Chronolog correctly

EXCLUSION CRITERIA: Any significant medical conditions other than mild asthma, allergic rhinitis, or eczema; respiratory tract infection, or an acute asthma exacerbation within the previous month; prednisone treatment, and emergency department visit or hospitalization within 3 months; life-threatening asthma episode or an adverse reaction to any B2-adrenergic agonist, or used salmeterol previously CRITERIA FOR RANDOMISATION DURING RUN-IN: N/A

\begin{tabular}{|c|c|c|}
\hline Interventions & \multicolumn{2}{|c|}{$\begin{array}{l}\text { LABA + ICS vs SAME dose of ICS } \\
\text { OUTCOMES measured at: day } 1 \text { and } 28 \\
\text { RUN IN PERIOD: Not specified } \\
\text { DOSE OF ICS DURING RUN-IN: Not reported } \\
\text { DOSE OPTIMISATION PERIOD: None } \\
\text { INTERVENTION PERIOD: } 28 \text { days } \\
\text { WASH OUT PERIOD: } 14 \text { days } \\
\text { TEST GROUP: Salmeterol } 50 \text { mcg once daily + BDP } 100-200 \text { mcg BID } \\
\text { CONTROL GROUP: BDP } 100-200 \text { mcg BID + placebo } \\
\text { DEVICE: Metered-dose inhaler and Nebulizer Chronolog device } \\
\text { NUMBER OF DEVICES: } 2 \\
\text { COMPLIANCE: Medication usage recorded in patient diary. A device inserted into } \\
\text { MDI recorded date, hour and minute of each inhalation } \\
\text { CO-TREATMENT: prn SABA (200 ug up to three times daily) except that albuterol } \\
\text { was not permitted } 8 \text { hours before each exercise test. If subjects had allergic rhinitis, } \\
\text { they were permitted to use pseudoephedrine (Sudafed) one to three times daily as } \\
\text { needed, except on the days when exercise tests were scheduled }\end{array}$} \\
\hline Outcomes & \multicolumn{2}{|c|}{$\begin{array}{l}\text { PULMONARY FUNCTION TEST: Exercise challenge (max \% fall in FEV1 from } \\
\text { pre-exercise baseline) } \\
\text { SYMPTOM SCORES: Symptoms } \\
\text { FUNCTIONAL STATUS: Rescue medication use; exacerbations requiring systemic } \\
\text { steroids } \\
\text { INFLAMMATORY MARKERS: Not reported } \\
\text { ADVERSE EFFECTS: Reported } \\
\text { WITHDRAWALS: Described } \\
\text { Primary outcome: Not specified }\end{array}$} \\
\hline Notes & \multicolumn{2}{|c|}{$\begin{array}{l}\text { Full-text publication } \\
\text { Funded by GSK } \\
\text { Confirmation of data and methodology obtained } \\
\text { User defined number: } 300 \text { ( } 1 / 2 \text { with BDP } 100 \text { bid; } 1 / 2 \text { with BDP } 200 \text { bid })\end{array}$} \\
\hline \multicolumn{3}{|l|}{ Risk of bias } \\
\hline Item & Authors' judgement & Description \\
\hline Adequate sequence generation? & Yes & Computer generated random numbers \\
\hline Allocation concealment? & Unclear & Information not available \\
\hline $\begin{array}{l}\text { Blinding? } \\
\text { All outcomes }\end{array}$ & Yes & Double-blind; double dummy \\
\hline $\begin{array}{l}\text { Incomplete outcome data } \\
\text { addressed? } \\
\text { All outcomes }\end{array}$ & Yes & All participants completed the study \\
\hline Free of selective reporting? & Yes & Data on OCS-treated exacerbations available \\
\hline Free of other bias? & Unclear & $\begin{array}{l}\text { Information on } \% \text { screening population } \\
\text { eligible not available }\end{array}$ \\
\hline
\end{tabular}

Stelmach 2007

Methods $\quad$ Parallel group single centre study in Poland




\begin{tabular}{|c|c|c|}
\hline Participants & \multicolumn{2}{|c|}{$\begin{array}{l}\text { \% ELIGIBLE OF SCREENED POPULATION: } 97 \\
\text { \% RUN-IN PARTICIPANTS RANDOMISED: Not reported } \\
\text { RANDOMISED: } 58 \text { (BUD/F: 29; BUD: 29) } \\
\text { WITHDRAWALS: } 0 \\
\text { AGE mean (range) or mean (SD): } 10 \text { years } \\
\text { SEVERITY: Moderate } \\
\text { BASELINE \% PRED. FEV1: } 94 \% \\
\text { BASELINE DOSE OF ICS: } 400 \mathrm{mcg} / \mathrm{d} \text { BDP equivalent } \\
\text { ASTHMA DURATION: } 4 \text { years } \\
\text { ATOPY (\%): } 100 \\
\text { ELIGIBILITY CRITERIA: 6-18 years; history of asthma requiring treatment with } \\
\text { ICS } \\
\text { EXCLUSION CRITERIA: Upper RTI in previous } 3 \text { weeks; sinus disease requiring } \\
\text { antibiotics within } 4 \text { weeks; oral steroids within } 4 \text { weeks of study entry; } \\
\text { immunotherapy } \\
\text { ELIGIBILITY CRITERIA DURING RUN IN: Not reported }\end{array}$} \\
\hline Interventions & \multicolumn{2}{|c|}{$\begin{array}{l}\text { ICS and LABA versus SAME DOSE ICS } \\
\text { OUTCOMES: } 8 \text { weeks } \\
\text { RUN IN PERIOD: } 4 \text { weeks } \\
\text { DOSE OPTIMISATION PERIOD: } \\
\text { INTERVENTION PERIOD: } 8 \text { weeks } \\
\text { TEST GROUP: Budesonide } 200 \mathrm{mcg}+\text { formoterol } 9 \mathrm{mcg} \text { via turbuhaler } \\
\text { CONTROL GROUP: Budesonide 200mcg daily via turbuhaler } \\
\text { NUMBER OF DEVICES: } 2 \\
\text { COMPLIANCE: Not assessed } \\
\text { CO-TREATMENT: prn SABA }\end{array}$} \\
\hline Outcomes & \multicolumn{2}{|c|}{$\begin{array}{l}\text { PULMONARY FUNCTION TEST: FEV1 predicted; FEF25-75; SRaw } \\
\text { SYMPTOM SCORES: Not reported } \\
\text { FUNCTIONAL STATUS: Not reported } \\
\text { INFLAMMATORY MARKERS: Not reported } \\
\text { ADVERSE EFFECTS: Not reported } \\
\text { WITHDRAWALS: Reported }\end{array}$} \\
\hline Notes & \multicolumn{2}{|c|}{$\begin{array}{l}\text { Full-text article } \\
\text { Funded by grant from Lodz University, Poland } \\
\text { Confirmation of data and methodology: Not obtained } \\
\text { User defined: } 200\end{array}$} \\
\hline \multicolumn{3}{|l|}{ Risk of bias } \\
\hline Item & Authors' judgement & Description \\
\hline Adequate sequence generation? & Yes & Computer-generated randomisation schedule \\
\hline Allocation concealment? & Unclear & Information not available \\
\hline $\begin{array}{l}\text { Blinding? } \\
\text { All outcomes }\end{array}$ & Yes & Double-blind; double dummy \\
\hline $\begin{array}{l}\text { Incomplete outcome data } \\
\text { addressed? } \\
\text { All outcomes }\end{array}$ & Yes & All completed \\
\hline Free of selective reporting? & Unclear & $\begin{array}{l}\text { Unclear whether data on OCS-treated exacerbations were } \\
\text { collected. Request for this information from study } \\
\text { investigator has not been successful }\end{array}$ \\
\hline Free of other bias? & Yes & $97 \%$ screening population eligible for study \\
\hline
\end{tabular}

Tal 2002

\begin{tabular}{ll}
\hline Methods & Parallel study, multicentre study (48 centers in 7 countries) \\
\hline Participants & Asymptomatic children \\
& \%ELIGIBLE OF SCREENED POPULATION: \\
& Not reported \\
& \%RUN-IN PARTICIPANTS RANDOMISED: \\
& Not reported \\
RANDOMISED: 286 (F+BDP: 148; BDP: 138) & WITHDRAWALS: F/BDP: 9; BDP: 9
\end{tabular}

Cochrane Database Syst Rev. Author manuscript; available in PMC 2014 September 19. 


\begin{tabular}{|c|c|c|}
\hline & \multicolumn{2}{|c|}{$\begin{array}{l}\text { AGE: mean (range): 11(4-17) } \\
\text { GENDER: (\%male): } 62 \\
\text { SEVERITY: Mild } \\
\text { BASELINE \% PRED. FEV1: } 75 \\
\text { BASELINE DOSE OF ICS: } 548 \\
\text { ASTHMA DURATION: } 6.8 \text { years } \\
\text { ATOPY (\%): Not reported } \\
\text { ELIGIBILITY CRITERIA: 4-17rs old; asthma diagnosed minimum } 6 \text { months; } \\
\text { FEV1 40-90\%predicted and >15\% reversibility in FEV1 within } 15 \text { minutes of } \\
\text { bronchodilator; constant dose ICS for prior } 6 \text { weeks ( }>400 \text { mcg budesonide } \\
\text { turbuhaler, >600mcg Budes-onide via MDI, >375 mcg fluticasone propionate or > } \\
\text { 600mcg CFC beclomethasone dipropionate) } \\
\text { EXCLUSION CRITERIA: Unstable asthma (defined as use of oral, parenteral or } \\
\text { rectal corticosteroids within } 30 \text { days of study commencement); respiratory tract } \\
\text { infection within previous } 4 \text { weeks; if they had known hypersensitivity to study } \\
\text { medications or inhaled lactose; use of inhaled ICS other than study medication not } \\
\text { allowed } \\
\text { CRITERIA FOR RANDOMISATION DURING RUN-IN: No other additional } \\
\text { criteria }\end{array}$} \\
\hline Interventions & \multicolumn{2}{|c|}{$\begin{array}{l}\text { LABA + ICS vs SAME dose of ICS } \\
\text { OUTCOMES measured at: } 4,8 \text { and } 12 \text { weeks } \\
\text { RUN IN PERIOD: } 2-4 \text { weeks } \\
\text { DOSE OF ICS DURING RUN-IN: BUD } 200 \text { bid } \\
\text { DOSE OPTIMISATION PERIOD: None } \\
\text { INTERVENTION PERIOD: } 12 \text { weeks } \\
\text { TEST GROUP: Formoterol } 12 \text { cg bid + BDP } 200 \text { mcg BID } \\
\text { CONTROL GROUP: BDP } 200 \text { mcg bid and placebo } \\
\text { DEVICE: Turbuhaler } \\
\text { NUMBER OF DEVICES: } 2 \\
\text { COMPLIANCE: Not reported } \\
\text { CO-TREATMENT: prn SABA. If subjects had allergic rhinitis, they were permitted } \\
\text { to use nasal corticosteroids; treatment with other asthma medication not permitted }\end{array}$} \\
\hline Outcomes & \multicolumn{2}{|c|}{$\begin{array}{l}\text { PULMONARY FUNCTION TEST: Am PEF*; pm PEF; FEV1 predicted } \\
\text { SYMPTOM SCORES: Daily and nocturnal on } 4 \text { point scale } \\
\text { FUNCTIONAL STATUS: Rescue medication use; night time awakening; } \\
\text { symptom-free days } \\
\text { INFLAMMATORY MARKERS: Not reported } \\
\text { ADVERSE EFFECTS: Reported } \\
\text { WITHDRAWALS: Described } \\
\text { Primary outcome* }\end{array}$} \\
\hline Notes & \multicolumn{2}{|c|}{$\begin{array}{l}\text { Full-text publication } \\
\text { Source of Funding Astra Zeneca } \\
\text { Confirmation of data and methodology obtained } \\
\text { User defined number: } 400\end{array}$} \\
\hline \multicolumn{3}{|l|}{ Risk of bias } \\
\hline Item & Authors' judgement & Description \\
\hline Adequate sequence generation? & Yes & Computer generated random numbers \\
\hline Allocation concealment? & Yes & $\begin{array}{l}\text { 'Individual treatment code envelopes were } \\
\text { provided for each subject.' }\end{array}$ \\
\hline $\begin{array}{l}\text { Blinding? } \\
\text { All outcomes }\end{array}$ & Yes & Double-blind; double dummy \\
\hline $\begin{array}{l}\text { Incomplete outcome data } \\
\text { addressed? } \\
\text { All outcomes }\end{array}$ & Unclear & $\begin{array}{l}\text { Explicit description of how ITT population } \\
\text { was composed was not presented: 'An } \\
\text { intention-to-treat analysis was used with all } \\
\text { available data.' }\end{array}$ \\
\hline Free of selective reporting? & Unclear & $\begin{array}{l}\text { Unclear whether data on OCS-treated } \\
\text { exacerbations were collected in the study. } \\
\text { Correspondence with study investigators has } \\
\text { not clarified this }\end{array}$ \\
\hline Free of other bias? & Unclear & $\begin{array}{l}\text { No information available on } \% \text { screening/run- } \\
\text { in populations eligible for the study }\end{array}$ \\
\hline
\end{tabular}

Teper 2005 


\begin{tabular}{|c|c|c|}
\hline Methods & \multicolumn{2}{|c|}{ Parallel group, single centre study } \\
\hline Participants & \multicolumn{2}{|c|}{$\begin{array}{l}\text { Mild-moderate asthmatic children } \\
\text { \%ELIGIBLE OF SCREENED POPULATION: Not reported } \\
\text { \%RUN-IN PARTICIPANTS RANDOMISED: Not reported } \\
\text { RANDOMISED: } 82 \text { (FP/SAL: 43; FP: 39) } \\
\text { WITHDRAWAL: Not reported } \\
\text { AGE mean: } 10 \text { years } \\
\text { GENDER (male\%): } 59 \\
\text { ASTHMA SEVERITY: Mild to moderate } \\
\text { BASELINE \% PRED. FEV1: } 95 \\
\text { BASELINE DOSE OF ICS (start of run in): Not reported } \\
\text { ASTHMA DURATION: Not reported } \\
\text { ATOPY(\%): Not reported } \\
\text { ELIGIBILITY CRITERIA: ATS diagnosed mild or moderate asthma; age 6-14 } \\
\text { years participants; FEV1 > 70 \% predicted; methacholine PC20 <2mcg/ml } \\
\text { EXCLUSION CRITERIA: Not reported } \\
\text { CRITERIA FOR RANDOMISATION DURING RUN-IN: Not reported }\end{array}$} \\
\hline Interventions & \multicolumn{2}{|c|}{$\begin{array}{l}\text { LABA + ICS versus SAME dose ICS } \\
\text { OUTCOMES: } 12 \text { months } \\
\text { RUN IN PERIOD: Unclear } \\
\text { DOSE OF ICS DURING RUN IN: Not reported } \\
\text { DOSE OPTIMISATION PERIOD: None reported } \\
\text { INTERVENTION PERIOD: } 12 \text { months } \\
\text { TEST GROUP: Combination fluticasone and salmeterol } 125 / 25 \text { bid } \\
\text { CONTROL GROUP: Fluticasone } 125 \mathrm{mcg} \text { bid } \\
\text { DEVICE: MDI (+ aerochamber) } \\
\text { NUMBER OF DEVICES: } 1 \\
\text { COMPLIANCE: Not reported } \\
\text { CO-TREATMENT: prn SABA }\end{array}$} \\
\hline Outcomes & \multicolumn{2}{|c|}{$\begin{array}{l}\text { PULMONARY FUNCTION TEST: FEV1 \% predicted } \\
\text { SYMPTOM SCORES: \% symptom-free days; \% symptom-free nights } \\
\text { FUNCTIONAL STATUS: \% SABA-free days } \\
\text { INFLAMMATORY MARKERS: PC20 } \\
\text { ADVERSE EFFECTS: Reported } \\
\text { WITHDRAWAL: Not reported } \\
\text { Primary outcome: not clear }\end{array}$} \\
\hline Notes & \multicolumn{2}{|c|}{$\begin{array}{l}\text { Unpublished conference abstract } \\
\text { Source of Funding: Not reported } \\
\text { Confirmation of data and methodology: Not obtained } \\
\text { User defined number: } 500\end{array}$} \\
\hline \multicolumn{3}{|l|}{ Risk of bias } \\
\hline Item & Authors' judgement & Description \\
\hline Adequate sequence generation? & Unclear & $\begin{array}{l}\text { Described as randomised; no other } \\
\text { information available }\end{array}$ \\
\hline Allocation concealment? & Unclear & Information not available \\
\hline $\begin{array}{l}\text { Blinding? } \\
\text { All outcomes }\end{array}$ & Unclear & $\begin{array}{l}\text { Double-blind; means by which assignment } \\
\text { to treatment group is masked is not } \\
\text { available }\end{array}$ \\
\hline $\begin{array}{l}\text { Incomplete outcome data } \\
\text { addressed? } \\
\text { All outcomes }\end{array}$ & Unclear & $\begin{array}{l}\text { No information provided on ITT } \\
\text { population }\end{array}$ \\
\hline Free of selective reporting? & Unclear & $\begin{array}{l}\text { Unclear whether data on OCS-treated } \\
\text { exacerbations were collected }\end{array}$ \\
\hline Free of other bias? & Unclear & $\begin{array}{l}\text { No information available on } \% \text { screening/ } \\
\text { run-in populations eligible for the study }\end{array}$ \\
\hline
\end{tabular}

Verberne 1998a 


\begin{tabular}{|c|c|}
\hline Methods & $\begin{array}{l}\text { Parallel group, multicentre study ( } 9 \text { centres). Three groups of which two are } \\
\text { considered in this review }\end{array}$ \\
\hline Participants & $\begin{array}{l}\text { Asthmatic Children } \\
\% \text { ELIGIBLE OF SCREENED POPULATION: Not reported } \\
\text { \%RUN-IN PARTICIPANTS } \\
\text { RANDOMISED: Not reported } \\
\text { RANDOMISED: } 117 \text { (BDP400 + Salm: 60; BDP400: 57) } \\
\text { WITHDRAWALS: BDP400 + Salm: 5; BDP400: } 4 \\
\text { AGE: mean (SD): } 11 \text { (2.6) years } \\
\text { GENDER (\% male): } 65 \\
\text { SEVERITY: Mild } \\
\text { BASELINE \% PRED.FEV1: } 88 \\
\text { BASELINE DOSE OF ICS (SD): } 489 \text { (153) } \\
\text { ASTHMA DURATION mean (SD): } 8.1 \text { (3.2) } \\
\text { ATOPY(\%): } 88 \\
\text { ELIGIBILITY CRITERIA: FEV1 between } 55 \text { and 90\% predicted or a FEV1/FVC } \\
\text { ratio of 50 to 75\%; >=10\% improvement in FEV1 after inhalation of salbutamol; } \\
\text { airway hyper-responsiveness to methacholine (PD20); ability to reproduce lung } \\
\text { function test; history of stable asthma for >= 1 month without exacerbation or } \\
\text { respiratory tract infection; use of inhaled steroids between } 200 \text { and } 800 \text { mg/day for } \\
\text { at least } 3 \text { months prior to the beginning of the study; } \\
\text { EXCLUSION CRITERIA: Operations for congenital heart disease, oesophageal } \\
\text { atresia, congenital or acquired anatomical malformation of the lungs or airways, } \\
\text { dyskinetic cilia syndrome; bronchiectasis; bronchopulmonary dysplasia; diabetes; } \\
\text { renal disease; other serious conditions which may influence the possibility of } \\
\text { continuation of the study; were using oral corticosteroids continuously or inhaled } \\
\text { corticosteroids at a dose of more than } 800 \text { mcg daily; were using B-blocking agents } \\
\text { or had used cromoglycate or nedocromil sodium within the previous two weeks; } \\
\text { were allergic to B-agonists; were pregnant or lactating, or females of childbearing } \\
\text { age who in the opinion of the supervising physician were not taking adequate } \\
\text { contraceptive precautions; an ongoing hyposensitising programme; inability to } \\
\text { follow therapy instructions, inability to inhale medications adequately or inability to } \\
\text { use peak flow meter. During study: non-compliance with respect to study } \\
\text { medication, completing the diary cards, clinic visits; withdrawal at own or } \\
\text { investigators discretion; total number of course of oral corticosteroids more than } \\
\text { allowed in study } \\
\text { CRITERIA FOR RANODOMISATION DURING RUN IN: No additional criteria }\end{array}$ \\
\hline Interventions & $\begin{array}{l}\text { LABA + ICS vs SAME dose ICS } \\
\text { OUTCOMES: Reported at 6,12,18,24, 30,36,42,48 and } 54 \text { weeks } \\
\text { RUN IN PERIOD: } 6 \text { weeks } \\
\text { DOSE OF ICS DURING RUN IN: BDP } 200 \text { bid } \\
\text { INTERVENTION PERIOD: } 54 \text { weeks } \\
\text { DOSE OPTIMISATION PERIOD: None } \\
\text { TEST GROUP: (Salm50 + BDP200) Salmeterol } 50 \text { mcg bid and Beclomethasone } \\
200 \text { mcg bid } \\
\text { CONTROL GROUP: (BDP } 200+\text { placebo) Beclomethasone } 200 \text { mcg bid + placebo } \\
\text { DEVICE: Rotadisks in combination with a diskhaler } \\
\text { NUMBER OF DEVICES: } 2 \\
\text { COMPLIANCE: Not reported } \\
\text { CO-TREATMENT: prn SABA }\end{array}$ \\
\hline Outcomes & $\begin{array}{l}\text { PULMONARY FUNCTION TEST: FEV1; am PEF; pm PEF; FVC } \\
\text { SYMPTOM SCORES: Asthma symptoms like wheezing, dyspnea, exercise induced } \\
\text { asthma and cough were scored in the morning and evening using a scale from } 1 \text { to } 3 \\
\text { FUNCTIONAL STATUS: Rescue medication use; exacerbation ( requiring } \\
\text { systemic steroids); height, body weight, heart rate, systolic and diastolic blood } \\
\text { pressure were measured } \\
\text { INFLAMMATORY MARKERS: Total IgE } \\
\text { ADVERSE EFFECTS: Reported } \\
\text { WITHDRAWALS: Reported } \\
\text { *primary outcome: airway calibre measured as FEV1 and airway responsiveness to } \\
\text { methacholine }\end{array}$ \\
\hline Notes & $\begin{array}{l}\text { Full-text publication } \\
\text { Funded by GSK } \\
\text { Confirmation of methodology and data obtained } \\
\text { User-defined number: } 400\end{array}$ \\
\hline \multicolumn{2}{|l|}{ Risk of bias } \\
\hline Item & Authors' judgement \\
\hline
\end{tabular}




\begin{tabular}{lll} 
Adequate sequence generation? & Yes & Computer generated random numbers \\
\hline Allocation concealment? & Yes & $\begin{array}{l}\text { Telephone notification of assignment } \\
\text { by coordinating centre }\end{array}$ \\
\hline $\begin{array}{l}\text { Blinding? } \\
\text { All outcomes }\end{array}$ & Yes & Double-blind; identical placebo used \\
\hline $\begin{array}{l}\text { Incomplete outcome data } \\
\text { addressed? } \\
\text { All outcomes }\end{array}$ & Unclear & $\begin{array}{l}\text { Not clear how population for primary } \\
\text { outcome. Incomplete diary card data } \\
\text { not included in analysis: }\end{array}$ \\
& & $\begin{array}{l}\text { Where patients failed to complete } \\
\text { their daily record cards for more than } \\
\text { 7d in any 14-d period such } \\
\text { assessments were not included in the } \\
\text { analysis. Otherwise, when there were } \\
\text { missing days in the record, pro rata } \\
\text { adjustment was made to give a 2-wk } \\
\text { assessment. }\end{array}$ \\
\hline Free of selective reporting? & Yes & $\begin{array}{l}\text { OCS-treated exacerbation data } \\
\text { available }\end{array}$ \\
\hline Free of other bias? & Unclear & $\begin{array}{l}\text { No information available on \% } \\
\text { screening/run-in populations eligible } \\
\text { for the study }\end{array}$ \\
\hline
\end{tabular}

Verberne 1998b

\begin{tabular}{|c|c|}
\hline Methods & See Verberne 1998a \\
\hline Participants & $\begin{array}{l}\text { As for Verberne 1998a, except for } \\
\text { RANDOMISED: } 120 \text { (BDP400 + Salm: 60; BDP800: 60) } \\
\text { WITHDRAWALS: BDP400 + Salm: 5; BDP800: } 6\end{array}$ \\
\hline Interventions & $\begin{array}{l}\text { LABA + ICS vs INCREASED dose ICS } \\
\text { OUTCOMES: reported at } 6,12,18,24,30,36,42,48 \text { and } 54 \\
\text { RUN IN PERIOD: } 6 \text { weeks } \\
\text { DOSE OF ICS DURING RUN-IN: BDP } 200 \text { bid } \\
\text { DOSE OPTIMISATION PERIOD: None } \\
\text { INTERVENTION PERIOD: } 54 \text { weeks } \\
\text { TEST GROUP: (Salm50 + BDP200): Salmeterol } 50 \mathrm{mcg} \text { bid + beclomethasone } \\
\text { dipropionate } 200 \text { mcg bid } \\
\text { CONTROL GROUP: (BDP400 + placebo): Beclomethasone dipropionate } 400 \\
\text { mcg/day + placebo } \\
\text { DEVICE: Rotadisks in combination with a diskhaler } \\
\text { NUMBER OF DEVICES: } 2 \\
\text { COMPLIANCE: Not reported } \\
\text { CO-TREATMENT: prn SABA }\end{array}$ \\
\hline Outcomes & $\begin{array}{l}\text { PULMONARY FUNCTION TEST: FEV1; am PEF; pm PEF; FVC } \\
\text { SYMPTOM SCORES: Asthma symptoms like wheezing, dyspnea, exercise } \\
\text { induced asthma and cough were scored in the morning and evening using a scale } \\
\text { from } 1 \text { to } 3 \\
\text { FUNCTIONAL STATUS: Rescue medication use; exacerbation ( requiring } \\
\text { systemic steroids); height, body weight, heart rate, systolic and diastolic blood } \\
\text { pressure were measured } \\
\text { INFLAMMATORY MARKERS: Total IgE } \\
\text { ADVERSE EFFECTS: Reported } \\
\text { WITHDRAWALS: Reported } \\
\text { *primary outcome: airway calibre measured as FEV1 and airway responsiveness to } \\
\text { methacholine }\end{array}$ \\
\hline Notes & $\begin{array}{l}\text { Full-text publication } \\
\text { Funded by GSK } \\
\text { Confirmation of methodology and data obtained } \\
\text { User-defined number: } 400\end{array}$ \\
\hline \multicolumn{2}{|l|}{ Risk of bias } \\
\hline Item & Authors' judgement \\
\hline
\end{tabular}




\begin{tabular}{lll} 
Adequate sequence generation? & Yes & See Verberne 1998a \\
\hline Allocation concealment? & Yes & See Verberne 1998a \\
\hline $\begin{array}{l}\text { Blinding? } \\
\text { All outcomes }\end{array}$ & Yes & See Verberne 1998a \\
\hline $\begin{array}{l}\text { Incomplete outcome data } \\
\text { addressed? }\end{array}$ & Unclear & See Verberne 1998a \\
\hline All outcomes & & \\
\hline Free of selective reporting? & Yes & See Verberne 1998a \\
\hline Free of other bias? & Unclear & See Verberne 1998a \\
\hline
\end{tabular}

Zimmerman 2004a

\begin{tabular}{|c|c|}
\hline Methods & $\begin{array}{l}\text { Parallel-group, multicentre study ( } 27 \text { centres in Canada). Three treatment arms } \\
\text { comparing LABA/ICS with } 2 \text { doses of LABA and ICS alone. Two groups will be } \\
\text { considered here and since the same control group is being used for both } \\
\text { comparisons half the control group will be applied to each }\end{array}$ \\
\hline Participants & $\begin{array}{l}\text { Children aged >= 6-11 years } \\
\text { \%ELIGIBLE OF SCREENED POPULATION: Not reported } \\
\% \text { RUN-IN PARTICIPANTS RANDOMISED: } 68 \\
\text { RANDOMISED: } 196 \text { (F + usual ICS bid: } 95 \text {; usual ICS: 101) } \\
\text { WITHDRAWALS: F + usual ICS: 7; usual ICS: } 16 \\
\text { Mean AGE years(range): } 9 \text { ( } 6 \text { to } 11 \text { ) } \\
\text { GENDER: (\%male): } 63 \\
\text { SEVERITY: Moderate } \\
\text { BASELINE FEV1 (\% Pred): } 77.4 \\
\text { BASELINE DOSE OF ICS: } 445 \\
\text { ASTHMA DURATION ( years): } 5.7 \\
\text { ATOPY (\%): Not reported } \\
\text { ELIGIBILITY CRITERIA: Aged >=12 years; clinical diagnosis of asthma } \\
\text { according to ATS criteria for at least } 12 \text { months; treated with ICS for at least } 3 \\
\text { month prior to entry; FEV1 between } 50-90 \% \text { predicted normal; >=15\% reversibility } \\
\text { after bronchodilator; asthma symptoms suggestive that additional therapy might be } \\
\text { needed; able to use peak flow meter and turbuhaler, answer questions form the } \\
\text { Pediatric Asthma Quality of Life Questionnaire and parent or guardian had to } \\
\text { complete a daily diary card } \\
\text { EXCLUSION CRITERIA: Systemic corticosteroids or anti-leukotrienes within } 30 \\
\text { days of study entry, astemizole within } 120 \text { days, sodium cromoglycate or ketotifen } \\
\text { within } 7 \text { days, salmeterol or formoterol within } 72 \text { hours or xanthines or } \\
\text { antihistamines within } 48 \text { hours; nasal corticosteroids and immunotherapy permitted } \\
\text { provided dose had been constant for at least } 30 \text { days and } 90 \text { days respectively prior } \\
\text { to study entry; smoking history } \\
\text { RANDOMISATION CRITERIA FOLLOWING RUN-IN: Post-bronchodilator } \\
\text { reversibility of at least } 12 \% \text { of the pre-bronchodilator value or at least } 9 \% \text { of } \\
\text { predicted normal or diurnal variability or at least } 15 \% \text { on any } 5 \text { of the last } 10 \text { days of } \\
\text { run-in; } 75124 \% \text { compliance with prescribed dose as assessed by diary card; } \\
\text { symptoms during the last } 10 \text { days of run-in (defined as having one or more of the } \\
\text { following: four or more inhalations of rescue medication; daytime symptoms on } 4 \text { or } \\
\text { more days, or night time awakening on } 1 \text { or more nights) }\end{array}$ \\
\hline Interventions & $\begin{array}{l}\text { LABA + ICS versus usual dose of ICS } \\
\text { OUTCOMES: Measured at trial entry and after } 4,8 \text { and } 12 \text { week intervals } \\
\text { RUN-IN PERIOD: } 2 \text { weeks } \\
\text { DOSE OF ICS DURING RUN-IN: Usual ICS } \\
\text { DOSE OPTIMISATION PERIOD: None } \\
\text { INTERVENTION PERIOD: } 12 \text { weeks } \\
\text { TEST GROUP: Usual dose ICS + Formoterol } 12 \text { mcgs bid } \\
\text { CONTROL GROUP: Usual dose ICS + placebo bid } \\
\text { DEVICE: Turbuhaler } \\
\text { NUMBER OF DEVICES: } 2 \\
\text { COMPLIANCE: Measured during run-in } \\
\text { CO-TREATMENT: prn SABA }\end{array}$ \\
\hline
\end{tabular}

Outcomes

PULMONARY FUNCTION TEST: am PEF*; pm PEF; FEV1 (Note: Mean value during treatment for 12 weeks reported rather than value at endpoint) SYMPTOM SCORES: Total asthma symptom score 
FUNCTIONAL STATUS: Rescue medication use; Pediatric asthma quality of life score

INFLAMMATORY MARKERS: Not described

ADVERSE EFFECTS: Described

WITHDRAWALS: Described

Primary outcome measure*

\begin{tabular}{|c|c|c|}
\hline Notes & \multicolumn{2}{|c|}{$\begin{array}{l}\text { Full-text publication } \\
\text { Supported by: not stated } \\
\text { Confirmation of methodology and data extraction not obtained } \\
\text { User defined number (mean ICS dose in LABA group in mcg/day of BDP- } \\
\text { equivalent): } 444\end{array}$} \\
\hline \multicolumn{3}{|l|}{ Risk of bias } \\
\hline Item & Authors' judgement & Description \\
\hline Adequate sequence generation? & Unclear & $\begin{array}{l}\text { Described as randomised; no other } \\
\text { information presented }\end{array}$ \\
\hline Allocation concealment? & Unclear & Information not available \\
\hline $\begin{array}{l}\text { Blinding? } \\
\text { All outcomes }\end{array}$ & Yes & Double-blind; double dummy \\
\hline $\begin{array}{l}\text { Incomplete outcome data } \\
\text { addressed? } \\
\text { All outcomes }\end{array}$ & Unclear & $\begin{array}{l}\text { Intention to treat population presented in the } \\
\text { study publication, but handling of missing } \\
\text { data not described }\end{array}$ \\
\hline Free of selective reporting? & Yes & $\begin{array}{l}\text { Exacerbations described as those requiring } \\
\text { OCS-treatment and those requiring increased } \\
\text { inhaled steroid. Separate OCS-treated } \\
\text { exacerbation data could not be extracted }\end{array}$ \\
\hline Free of other bias? & Yes & $68 \%$ of screening population randomised \\
\hline
\end{tabular}

\section{Zimmerman 2004b}

\begin{tabular}{|c|c|c|}
\hline Methods & \multicolumn{2}{|l|}{ See Zimmerman 2004a } \\
\hline Participants & \multicolumn{2}{|c|}{$\begin{array}{l}\text { See Zimmerman 2004a, except for } \\
\text { RANDOMISED: } 196 \text { (F + Usual ICS: 95; usual ICS: 101) } \\
\text { WITHDRAWALS: F + usual ICS: 7; usual ICS: } 16\end{array}$} \\
\hline Interventions & \multicolumn{2}{|c|}{$\begin{array}{l}\text { As for Zimmerman 2004a, except for: } \\
\text { TEST GROUP: Usual dose ICS + Formoterol } 6 \text { mcgs bid }\end{array}$} \\
\hline Outcomes & \multicolumn{2}{|l|}{ See Zimmerman 2004a } \\
\hline Notes & \multicolumn{2}{|l|}{ See Zimmerman 2004a } \\
\hline \multicolumn{3}{|l|}{ Risk of bias } \\
\hline Item & Authors' judgement & Description \\
\hline Adequate sequence generation? & Unclear & See Zimmerman 2004a \\
\hline Allocation concealment? & Unclear & See Zimmerman 2004a \\
\hline $\begin{array}{l}\text { Blinding? } \\
\text { All outcomes }\end{array}$ & Yes & See Zimmerman 2004a \\
\hline $\begin{array}{l}\text { Incomplete outcome data addressed? } \\
\text { All outcomes }\end{array}$ & Unclear & See Zimmerman 2004a \\
\hline Free of selective reporting? & Yes & See Zimmerman 2004a \\
\hline Free of other bias? & Yes & See Zimmerman 2004a \\
\hline
\end{tabular}

LABA: Long-acting beta agonist (salmeterol or formoterol);

Sal: Salmeterol;

F: Formoterol;

Cochrane Database Syst Rev. Author manuscript; available in PMC 2014 September 19. 
ICS: Inhaled corticosteroid;

BUD: Budesonide;

FP: Fluticasone;

BID: twice per day;

QD: once per day

\section{Characteristics of excluded studies [ordered by study ID]}

\begin{tabular}{|c|c|}
\hline Study & Reason for exclusion \\
\hline Aldington 2006 & Inadequate duration \\
\hline Aubier 1999 & Study conducted in adults \\
\hline Bergmann 2004 & Not exclusively children \\
\hline Borker 2005 & No LABA alone \\
\hline Boulet 2003 & Adult study \\
\hline Bousquet 2005 & No LABA alone \\
\hline Bracamonte 2005 & Comparison of devices \\
\hline Bruce 2005 & Review article \\
\hline Bruggenjurgen 2005 & Not exclusively paediatric \\
\hline Buchvald 2002 & No ICS alone \\
\hline Buhl 2004 & Adjustable dosing in adults \\
\hline Caffey 2005 & No LABA alone \\
\hline Chopra 2005 & No ICS alone \\
\hline Chuchalin 2005 & No ICS alone \\
\hline Cowan 2004 & Not RCT \\
\hline Daviskas 2005 & No ICS alone \\
\hline Delaronde 2005 & No assessment of asthma control \\
\hline Dubus 2003 & No LABA \\
\hline Emeryk 2003 & Comparison of devices \\
\hline Everden 2004 & Not placebo-controlled \\
\hline Fardon 2005 & No LABA \\
\hline Grady 1995 & Not exclusively children \\
\hline Holgate 2004 & Study of Xolair \\
\hline Holt 2005 & Not exclusively children \\
\hline Ilowite 2004 & Adult study \\
\hline Jenkins 2005 & Adult study \\
\hline Karaman 2007 & No prior ICS exposure \\
\hline Lara-Perez 2005 & No ICS alone \\
\hline Levy 2005 & $\begin{array}{l}\text { Co-treatment with ICS in only } 3 / 4 \text { of participants. Study primarily interested in efficacy of } \\
\text { formoterol on top of usual therapy. ICS dosing not standardised }\end{array}$ \\
\hline LOCCS & Adult study \\
\hline Matthys 2004 & Open study \\
\hline Miraglia 2007 & No prior exposure to ICS \\
\hline
\end{tabular}




\begin{tabular}{|c|c|}
\hline Mitchell 2005 & Not LABA \\
\hline Mitra 2003 & No concurrent ICS \\
\hline Morice 2005 & Not exclusively children \\
\hline Morice 2005a & Not exclusively children \\
\hline Murray 2004 & Not exclusively children \\
\hline Nathan 2005 & Not exclusively children \\
\hline Nelson 2006 & Not exclusively children \\
\hline Nguyen 2005 & Not RCT \\
\hline O‘Byrne 2001 & Adolescents and adults \\
\hline Pearlman 2004 & Study in adults \\
\hline Peroni 2005 & No ICS alone \\
\hline Pijnenburg 2005 & No LABA \\
\hline Prieto 2005 & Not exclusively children \\
\hline Renzi 2005 & Not exclusively children \\
\hline SAM30002 & Not exclusively children \\
\hline SAM40101 & Inadequate duration \\
\hline SAS30021 & Steroid naive children \\
\hline Schauer 2003 & No ICS alone \\
\hline Scicchitano 2004a & Study in adults \\
\hline Selroos 2004 & No LABA \\
\hline SFCF3001 & Different devices \\
\hline SFCF3002 & Different devices \\
\hline Sienra-Monge 2004 & Not RCT \\
\hline Sorkness 2007 & Mixed population at baseline \\
\hline Stelmach 2008 & Steroids were stopped for 4 weeks prior to study visit \\
\hline Storms 2004 & Study in adults \\
\hline van den Toorn 2005 & No ICS alone \\
\hline Vogelmeier 2005 & No ICS alone \\
\hline Von Berg 2003 & No concurrent ICS. \\
\hline Weiler 2005 & Study in adults \\
\hline You-Ning 2005 & Study in adults; no ICS alone \\
\hline
\end{tabular}

EIB: Exercise-induced bronchoconstriction; LABA: long-acting beta-agonist 
DATA AND ANALYSES

Comparison 1

Long-acting beta2 versus placebo: both groups receiving similar dose ICS

\begin{tabular}{|c|c|c|c|c|}
\hline $\begin{array}{l}\text { Outcome or subgroup } \\
\text { title }\end{array}$ & No. of studies & No. of participants & Statistical method & Effect size \\
\hline $\begin{array}{l}1 \text { \# patients with } \\
\text { exacerbations requiring } \\
\text { systemic steroids }\end{array}$ & 8 & 1084 & $\begin{array}{c}\text { Risk Ratio (M-H, Fixed, } \\
95 \% \text { CI) }\end{array}$ & $0.92[0.60,1.40]$ \\
\hline $\begin{array}{l}1.1 \text { Mean baseline } \\
\text { FEV1 }>/=80 \% \text { of } \\
\text { predicted }\end{array}$ & 4 & 375 & $\begin{array}{c}\text { Risk Ratio (M-H, Fixed, } \\
95 \% \text { CI) }\end{array}$ & $0.87[0.47,1.64]$ \\
\hline $\begin{array}{l}1.2 \text { Mean baseline } \\
\text { FEV1 } 61-79 \% \text { of } \\
\text { predicted }\end{array}$ & 2 & 230 & $\begin{array}{c}\text { Risk Ratio (M-H, Fixed, } \\
95 \% \text { CI) }\end{array}$ & $0.89[0.48,1.64]$ \\
\hline $\begin{array}{l}\text { 1.3 Mean baseline } \\
\text { FEV1 not reported }\end{array}$ & 2 & 479 & $\begin{array}{c}\text { Risk Ratio (M-H, Fixed, } \\
95 \% \mathrm{CI})\end{array}$ & $1.54[0.26,9.09]$ \\
\hline $\begin{array}{l}2 \text { \# patients with } \\
\text { exacerbations requiring } \\
\text { hospitalisation }\end{array}$ & 6 & 1266 & $\begin{array}{l}\text { Risk Ratio (M-H, Fixed, } \\
95 \% \mathrm{CI})\end{array}$ & $1.65[0.83,3.25]$ \\
\hline $\begin{array}{l}2.1 \text { Mean baseline } \\
\text { FEV1 }>/=80 \% \text { of } \\
\text { predicted }\end{array}$ & 2 & 139 & $\begin{array}{c}\text { Risk Ratio (M-H, Fixed, } \\
95 \% \mathrm{CI})\end{array}$ & $0.99[0.18,5.39]$ \\
\hline $\begin{array}{l}2.2 \text { Mean baseline } \\
\text { FEV1 } 61-79 \% \text { of } \\
\text { predicted }\end{array}$ & 3 & 772 & $\begin{array}{c}\text { Risk Ratio (M-H, Fixed, } \\
95 \% \mathrm{CI})\end{array}$ & $1.81[0.86,3.82]$ \\
\hline $\begin{array}{l}2.3 \text { Mean baseline } \\
\text { FEV1 not reported }\end{array}$ & 1 & 355 & $\begin{array}{c}\text { Risk Ratio (M-H, Fixed, } \\
95 \% \mathrm{CI})\end{array}$ & Not estimable \\
\hline 3 Serious adverse events & 17 & 4165 & $\begin{array}{c}\text { Risk Ratio (M-H, Fixed, } \\
95 \% \mathrm{CI})\end{array}$ & $1.16[0.73,1.85]$ \\
\hline $\begin{array}{l}3.1 \text { Mean baseline } \\
\text { FEV1 }>/=80 \% \text { of } \\
\text { predicted }\end{array}$ & 9 & 2041 & $\begin{array}{c}\text { Risk Ratio (M-H, Fixed, } \\
95 \% \mathrm{CI})\end{array}$ & $0.87[0.42,1.80]$ \\
\hline $\begin{array}{l}\text { 3.2 Mean baseline } \\
\text { FEV1 61-79\% of } \\
\text { predicted }\end{array}$ & 5 & 1283 & $\begin{array}{c}\text { Risk Ratio (M-H, Fixed, } \\
95 \% \mathrm{CI})\end{array}$ & $1.39[0.74,2.59]$ \\
\hline $\begin{array}{l}\text { 3.3 Mean baseline } \\
\text { FEV1 not reported }\end{array}$ & 3 & 841 & $\begin{array}{c}\text { Risk Ratio (M-H, Fixed, } \\
95 \% \mathrm{CI})\end{array}$ & $2.0[0.18,21.86]$ \\
\hline 4 Total \# withdrawals & 21 & 4295 & $\begin{array}{c}\text { Risk Ratio (M-H, Fixed, } \\
95 \% \mathrm{CI})\end{array}$ & $0.79[0.67,0.93]$ \\
\hline $\begin{array}{l}\text { 4.1 Mean baseline } \\
\text { FEV1 >/=80\% of } \\
\text { predicted }\end{array}$ & 11 & 2126 & $\begin{array}{c}\text { Risk Ratio (M-H, Fixed, } \\
95 \% \mathrm{CI})\end{array}$ & $0.80[0.63,1.02]$ \\
\hline $\begin{array}{l}\text { 4.2 Mean baseline } \\
\text { FEV1 } 61-79 \% \text { of } \\
\text { predicted }\end{array}$ & 6 & 1315 & $\begin{array}{c}\text { Risk Ratio (M-H, Fixed, } \\
95 \% \mathrm{CI})\end{array}$ & $0.88[0.68,1.15]$ \\
\hline $\begin{array}{l}\text { 4.3 Mean baseline } \\
\text { FEV1 not reported }\end{array}$ & 4 & 854 & $\begin{array}{c}\text { Risk Ratio (M-H, Fixed, } \\
95 \% \mathrm{CI})\end{array}$ & $0.56[0.35,0.91]$ \\
\hline $\begin{array}{l}5 \# \text { withdrawals due to } \\
\text { poor asthma control or } \\
\text { exacerbation }\end{array}$ & 9 & 1615 & $\begin{array}{c}\text { Risk Ratio (M-H, Fixed, } \\
95 \% \mathrm{CI})\end{array}$ & $0.79[0.42,1.48]$ \\
\hline $\begin{array}{l}5.1 \text { Mean baseline } \\
\text { FEV } 1>/=80 \% \text { of } \\
\text { predicted }\end{array}$ & 3 & 342 & $\begin{array}{c}\text { Risk Ratio (M-H, Fixed, } \\
95 \% \mathrm{CI})\end{array}$ & $0.54[0.15,1.92]$ \\
\hline $\begin{array}{l}5.2 \text { Mean baseline } \\
\text { FEV1 } 61-79 \% \text { of } \\
\text { predicted }\end{array}$ & 4 & 794 & $\begin{array}{c}\text { Risk Ratio (M-H, Fixed, } \\
95 \% \mathrm{CI})\end{array}$ & $0.78[0.34,1.81]$ \\
\hline
\end{tabular}




\begin{tabular}{|c|c|c|c|c|}
\hline $\begin{array}{l}\text { Outcome or subgroup } \\
\text { title }\end{array}$ & No. of studies & No. of participants & Statistical method & Effect size \\
\hline $\begin{array}{l}5.3 \text { Mean baseline } \\
\text { FEV1 not reported }\end{array}$ & 2 & 479 & $\begin{array}{c}\text { Risk Ratio (M-H, Fixed, } \\
95 \% \text { CI) }\end{array}$ & $1.36[0.31,5.98]$ \\
\hline $\begin{array}{l}6 \# \text { withdrawals due to } \\
\text { serious non-respiratory } \\
\text { event }\end{array}$ & 2 & & $\begin{array}{l}\text { Risk Ratio (M-H, } \\
\text { Random, 95\% CI) }\end{array}$ & Totals not selected \\
\hline $\begin{array}{l}\text { 6.1 Mean baseline } \\
\text { FEV1 } 61-79 \% \text { of } \\
\text { predicted }\end{array}$ & 2 & & $\begin{array}{l}\text { Risk Ratio (M-H, } \\
\text { Random, 95\% CI) }\end{array}$ & Not estimable \\
\hline $\begin{array}{l}7 \text { \# withdrawals due to } \\
\text { adverse events }\end{array}$ & 19 & 4419 & $\begin{array}{c}\text { Risk Ratio (M-H, Fixed, } \\
95 \% \text { CI) }\end{array}$ & $0.78[0.52,1.19]$ \\
\hline $\begin{array}{l}\quad 7.1 \text { Mean baseline } \\
\text { FEV } 1>/=80 \% \text { of } \\
\text { predicted }\end{array}$ & 9 & 2052 & $\begin{array}{c}\text { Risk Ratio (M-H, Fixed, } \\
95 \% \text { CI) }\end{array}$ & $0.62[0.34,1.13]$ \\
\hline $\begin{array}{l}\text { 7.2 Mean baseline } \\
\text { FEV1 } 61-79 \% \text { of } \\
\text { predicted }\end{array}$ & 7 & 1585 & $\begin{array}{c}\text { Risk Ratio (M-H, Fixed, } \\
95 \% \text { CI) }\end{array}$ & $1.40[0.68,2.87]$ \\
\hline $\begin{array}{l}\text { 7.3 Mean baseline } \\
\text { FEV1 not reported }\end{array}$ & 3 & 782 & $\begin{array}{c}\text { Risk Ratio (M-H, Fixed, } \\
\text { 95\% CI) }\end{array}$ & $0.37[0.11,1.25]$ \\
\hline $\begin{array}{l}8 \text { Change in FEV1 at } \\
\text { endpoint stratifying on } \\
\text { baseline FEV1 }\end{array}$ & 9 & 1235 & Litres (Random, 95\% CI) & $0.08[0.06,0.11]$ \\
\hline $\begin{array}{l}\quad 8.1 \text { Mean Baseline } \\
\text { FEV1 }>/=80 \% \text { of } \\
\text { predicted }\end{array}$ & 5 & 808 & Litres (Random, 95\% CI) & $0.09[0.04,0.15]$ \\
\hline $\begin{array}{l}\text { 8.2 Mean Baseline } \\
\text { FEV1 61-79\% of } \\
\text { predicted }\end{array}$ & 4 & 427 & Litres (Random, 95\% CI) & $0.08[0.05,0.11]$ \\
\hline $\begin{array}{l}\text { 8.3 Mean Baseline } \\
\text { FEV1 not reported }\end{array}$ & 0 & 0 & Litres (Random, 95\% CI) & Not estimable \\
\hline $\begin{array}{l}9 \text { Change in FEV1 at } \\
\text { endpoint (\% predicted) } \\
\text { stratifying on baseline } \\
\text { FEV1 }\end{array}$ & 5 & 476 & $\begin{array}{l}\text { Mean Difference (IV, } \\
\text { Fixed, 95\% CI) }\end{array}$ & $2.35[0.07,4.64]$ \\
\hline $\begin{array}{l}9.1 \text { Mean baseline } \\
\text { FEV1 }>/=80 \% \\
\text { predicted }\end{array}$ & 2 & 156 & $\begin{array}{l}\text { Mean Difference (IV, } \\
\text { Fixed, 95\% CI) }\end{array}$ & $3.20[0.07,6.33]$ \\
\hline $\begin{array}{l}9.2 \text { Mean Baseline } \\
\text { FEV1 } 61-79 \% \text { of } \\
\text { predicted }\end{array}$ & 2 & 238 & $\begin{array}{l}\text { Mean Difference (IV, } \\
\text { Fixed, 95\% CI) }\end{array}$ & $3.35[-1.50,8.20]$ \\
\hline $\begin{array}{l}\text { 9.3 Mean Baseline } \\
\text { FEV1 not reported }\end{array}$ & 1 & 82 & $\begin{array}{l}\text { Mean Difference (IV, } \\
\text { Fixed, 95\% CI) }\end{array}$ & $-0.40[-5.03,4.23]$ \\
\hline $\begin{array}{l}10 \mathrm{FEV} 1 \text { predicted at } \\
\text { endpoint stratifying on } \\
\text { baseline FEV1 }\end{array}$ & 4 & 634 & $\%$ (Random, 95\% CI) & $3.56[1.83,5.28]$ \\
\hline $\begin{array}{l}10.1 \text { Mean Baseline } \\
\text { FEV1 }>/=80 \% \text { of } \\
\text { predicted }\end{array}$ & 1 & 58 & $\%$ (Random, 95\% CI) & $1.1[-4.74,6.94]$ \\
\hline $\begin{array}{l}\text { 10.2 Mean Baseline } \\
\text { FEV1 } 61-79 \% \text { of } \\
\text { predicted }\end{array}$ & 3 & 576 & $\%$ (Random, 95\% CI) & $3.79[1.99,5.60]$ \\
\hline $\begin{array}{l}\text { 10.3 Mean Baseline } \\
\text { FEV1 not reported }\end{array}$ & 0 & 0 & $\%$ (Random, 95\% CI) & Not estimable \\
\hline $\begin{array}{l}11 \text { Change in FEV1 (L } \\
\text { or \% pred) stratifying on } \\
\text { trial duration }\end{array}$ & 11 & & $\begin{array}{l}\text { Std. Mean Difference } \\
\quad \text { (Fixed, 95\% CI) }\end{array}$ & Subtotals only \\
\hline
\end{tabular}




\begin{tabular}{|c|c|c|c|c|}
\hline $\begin{array}{l}\text { Outcome or subgroup } \\
\text { title }\end{array}$ & No. of studies & No. of participants & Statistical method & Effect size \\
\hline $\begin{array}{l}11.1 \text { Change in FEV1 } \\
\text { (L) or (\% predicted) at } 6 \\
+/-2 \text { weeks of treatment }\end{array}$ & 1 & & $\begin{array}{l}\text { Std. Mean Difference } \\
\quad \text { (Fixed, 95\% CI) }\end{array}$ & $0.31[-0.31,0.93]$ \\
\hline $\begin{array}{l}11.2 \text { Change in FEV1 } \\
\text { (L) or ( } \% \text { predicted) at } \\
12+/-4 \text { weeks of } \\
\text { treatment }\end{array}$ & 10 & & $\begin{array}{l}\text { Std. Mean Difference } \\
\quad \text { (Fixed, 95\% CI) }\end{array}$ & $0.32[0.23,0.42]$ \\
\hline $\begin{array}{l}11.3 \text { Change in FEV1 } \\
\text { (L) or }(\% \text { predicted) at } \\
24+/-4 \text { weeks of } \\
\text { treatment }\end{array}$ & 2 & & $\begin{array}{l}\text { Std. Mean Difference } \\
\quad \text { (Fixed, 95\% CI) }\end{array}$ & $0.36[0.13,0.60]$ \\
\hline $\begin{array}{l}\text { 11.4 Change in FEV1 } \\
\text { (L) or ( } \% \text { predicted) at } \\
52+/-4 \text { weeks of } \\
\text { treatment }\end{array}$ & 0 & & $\begin{array}{l}\text { Std. Mean Difference } \\
\quad \text { (Fixed, 95\% CI) }\end{array}$ & Not estimable \\
\hline $\begin{array}{l}12 \text { End of treatment } \\
\text { FEV1 (L) }\end{array}$ & 3 & 901 & $\begin{array}{l}\text { Mean Difference (IV, } \\
\text { Fixed, 95\% CI) }\end{array}$ & $-0.54[-0.61,-0.47]$ \\
\hline $\begin{array}{l}12.1 \text { Mean Baseline } \\
\text { FEV1 }>/=80 \% \text { of } \\
\text { predicted }\end{array}$ & 2 & 615 & $\begin{array}{l}\text { Mean Difference (IV, } \\
\text { Fixed, 95\% CI) }\end{array}$ & $-0.54[-0.61,-0.47]$ \\
\hline $\begin{array}{l}\text { 12.2 Mean baseline } \\
\text { FEV1 } 61-79 \% \text { of } \\
\text { predicted }\end{array}$ & 1 & 286 & $\begin{array}{l}\text { Mean Difference (IV, } \\
\text { Fixed, 95\% CI) }\end{array}$ & Not estimable \\
\hline $\begin{array}{l}13 \text { Change in PEF } \\
\text { variability at endpoint }\end{array}$ & 1 & & $\begin{array}{l}\text { Mean Difference (IV, } \\
\text { Fixed, 95\% CI) }\end{array}$ & Totals not selected \\
\hline $\begin{array}{l}\text { 13.1 Mean baseline } \\
\text { FEV1 } 61-79 \% \text { of } \\
\text { predicted }\end{array}$ & 1 & & $\begin{array}{l}\text { Mean Difference (IV, } \\
\text { Fixed, 95\% CI) }\end{array}$ & Not estimable \\
\hline $\begin{array}{l}14 \text { Change in morning } \\
\text { PEF at endpoint }\end{array}$ & 14 & 2934 & $\mathrm{~L} / \mathrm{min}$ (Fixed, 95\% CI) & $10.38[8.23,12.52]$ \\
\hline $\begin{array}{l}14.1 \text { Mean Baseline } \\
\text { FEV1 }>/=80 \% \text { of } \\
\text { predicted }\end{array}$ & 8 & 1870 & $\mathrm{~L} / \mathrm{min}$ (Fixed, 95\% CI) & $11.23[8.31,14.15]$ \\
\hline $\begin{array}{l}\text { 14.2 Mean Baseline } \\
\text { FEV1 61-79\% of } \\
\text { predicted }\end{array}$ & 5 & 713 & $\mathrm{~L} / \mathrm{min}$ (Fixed, 95\% CI) & $9.99[6.58,13.41]$ \\
\hline $\begin{array}{l}\text { 14.3 Mean Baseline } \\
\text { FEV1 not reported }\end{array}$ & 1 & 351 & $\mathrm{~L} / \mathrm{min}$ (Fixed, 95\% CI) & $5.7[-2.62,14.02]$ \\
\hline $\begin{array}{l}15 \text { Morning PEF at } \\
\text { endpoint }\end{array}$ & 3 & 459 & $\mathrm{~L} / \mathrm{min}$ (Fixed, $95 \% \mathrm{CI}$ ) & $8.64[2.53,14.75]$ \\
\hline $\begin{array}{l}15.1 \text { Mean Baseline } \\
\text { FEV1 }>/=80 \% \text { of } \\
\text { predicted }\end{array}$ & 0 & 0 & $\mathrm{~L} / \mathrm{min}$ (Fixed, 95\% CI) & Not estimable \\
\hline $\begin{array}{l}\text { 15.2 Mean Baseline } \\
\text { FEV1 61-79\% of } \\
\text { predicted }\end{array}$ & 3 & 459 & $\mathrm{~L} / \mathrm{min}$ (Fixed, 95\% CI) & $8.64[2.53,14.75]$ \\
\hline $\begin{array}{l}16 \text { Change in morning } \\
\text { PEF ( } \% \text { predicted) }\end{array}$ & 1 & & $\%$ (Fixed, $95 \% \mathrm{CI}$ ) & Totals not selected \\
\hline $\begin{array}{l}16.1 \text { Mean Baseline } \\
\text { FEV1 }>/=80 \% \text { of } \\
\text { predicted }\end{array}$ & 0 & & $\%$ (Fixed, $95 \% \mathrm{CI}$ ) & Not estimable \\
\hline $\begin{array}{l}\text { 16.2 Mean Baseline } \\
\text { FEV1 61-79\% of } \\
\text { predicted }\end{array}$ & 1 & & $\%$ (Fixed, 95\% CI) & Not estimable \\
\hline $\begin{array}{l}\text { 16.3 Mean Baseline } \\
\text { FEV1 not reported }\end{array}$ & 0 & & $\%$ (Fixed, $95 \% \mathrm{CI}$ ) & Not estimable \\
\hline
\end{tabular}




\begin{tabular}{|c|c|c|c|c|}
\hline $\begin{array}{l}\text { Outcome or subgroup } \\
\text { title }\end{array}$ & No. of studies & No. of participants & Statistical method & Effect size \\
\hline $\begin{array}{l}17 \text { Change in evening } \\
\text { PEF at endpoint }\end{array}$ & 11 & 2636 & L/min (Fixed, 95\% CI) & $9.37[6.96,11.79]$ \\
\hline $\begin{array}{l}\text { 17.1 Mean Baseline } \\
\text { FEV1 >/= } 80 \% \text { of } \\
\text { predicted }\end{array}$ & 7 & 1480 & L/min (Fixed, 95\% CI) & $9.38[6.29,12.47]$ \\
\hline $\begin{array}{l}\text { 17.2 Mean Baseline } \\
\text { FEV1 61-79\% of } \\
\text { predicted }\end{array}$ & 3 & 805 & L/min (Fixed, 95\% CI) & $10.46[6.15,14.78]$ \\
\hline $\begin{array}{l}\text { 17.3 Mean Baseline } \\
\text { FEV1 not reported }\end{array}$ & 1 & 351 & L/min (Fixed, 95\% CI) & $5.0[-3.58,13.58]$ \\
\hline $\begin{array}{l}18 \text { Change in evening } \\
\text { PEF ( } \% \text { predicted) }\end{array}$ & 1 & & $\%$ (Fixed, 95\% CI) & Totals not selected \\
\hline $\begin{array}{l}\text { 18.1 Mean Baseline } \\
\text { FEV1 >/= } 80 \% \text { of } \\
\text { predicted }\end{array}$ & 0 & & $\%$ (Fixed, 95\% CI) & Not estimable \\
\hline $\begin{array}{l}\text { 18.2 Mean Baseline } \\
\text { FEV1 } 61-79 \% \text { of } \\
\text { predicted }\end{array}$ & 1 & & $\%$ (Fixed, 95\% CI) & Not estimable \\
\hline $\begin{array}{l}\text { 18.3 Mean Baseline } \\
\text { FEV1 not reported }\end{array}$ & 0 & & $\%$ (Fixed, 95\% CI) & Not estimable \\
\hline $\begin{array}{l}19 \text { Change in clinic PEF } \\
(\mathrm{L} / \mathrm{min})\end{array}$ & 1 & & $\begin{array}{l}\text { Mean Difference (IV, } \\
\text { Fixed, 95\% CI) }\end{array}$ & Totals not selected \\
\hline $\begin{array}{l}\text { 19.1 Mean baseline } \\
\text { FEV1 not reported }\end{array}$ & 1 & & $\begin{array}{l}\text { Mean Difference (IV, } \\
\text { Fixed, 95\% CI) }\end{array}$ & Not estimable \\
\hline $\begin{array}{l}20 \text { Change in \# daytime } \\
\text { rescue inhalations (puffs } \\
\text { per day) at endpoint }\end{array}$ & 5 & 1278 & $\begin{array}{l}\text { Mean Difference (IV, } \\
\text { Random, 95\% CI) }\end{array}$ & $-0.07[-0.14,0.01]$ \\
\hline $\begin{array}{l}\text { 20.1 Mean baseline } \\
\text { FEV1 61-79\% of } \\
\text { predicted }\end{array}$ & 5 & 1278 & $\begin{array}{l}\text { Mean Difference (IV, } \\
\text { Random, 95\% CI) }\end{array}$ & $-0.07[-0.14,0.01]$ \\
\hline $\begin{array}{l}\text { 20.2 Mean Baseline } \\
\text { FEV1 not reported }\end{array}$ & 0 & 0 & $\begin{array}{l}\text { Mean Difference (IV, } \\
\text { Random, 95\% CI) }\end{array}$ & Not estimable \\
\hline $\begin{array}{l}21 \% \text { days without } \\
\text { bronchodilator usage }\end{array}$ & 3 & 712 & $\begin{array}{l}\text { Mean Difference (IV, } \\
\text { Fixed, 95\% CI) }\end{array}$ & $-0.56[-2.84,1.73]$ \\
\hline $\begin{array}{l}21.1 \text { Mean baseline } \\
\text { FEV } 1>/=80 \% \\
\text { predicted }\end{array}$ & 2 & 630 & $\begin{array}{l}\text { Mean Difference (IV, } \\
\text { Fixed, 95\% CI) }\end{array}$ & $0.01[-3.45,3.47]$ \\
\hline $\begin{array}{l}\text { 21.2 Mean baseline } \\
\text { FEV1 not reported }\end{array}$ & 1 & 82 & $\begin{array}{l}\text { Mean Difference (IV, } \\
\text { Fixed, 95\% CI) }\end{array}$ & $-1.0[-4.04,2.04]$ \\
\hline $\begin{array}{l}22 \text { Mean change in } \\
\text { asthma symptom score }\end{array}$ & 4 & 1119 & $\begin{array}{l}\text { Std. Mean Difference (IV, } \\
\text { Fixed, 95\% CI) }\end{array}$ & $-0.04[-0.16,0.08]$ \\
\hline $\begin{array}{l}22.1 \text { Mean baseline } \\
\text { FEV1 }>/=80 \% \text { of } \\
\text { predicted }\end{array}$ & 4 & 1119 & $\begin{array}{l}\text { Std. Mean Difference (IV, } \\
\text { Fixed, 95\% CI) }\end{array}$ & $-0.04[-0.16,0.08]$ \\
\hline $\begin{array}{l}23 \text { Change in \# } \\
\text { nighttime rescue } \\
\text { inhalations at endpoint }\end{array}$ & 1 & & $\begin{array}{l}\text { Mean Difference (IV, } \\
\text { Random, 95\% CI) }\end{array}$ & Totals not selected \\
\hline $\begin{array}{l}23.1 \text { Mean baseline } \\
\text { FEV1 } 61-79 \% \text { of } \\
\text { predicted }\end{array}$ & 1 & & $\begin{array}{l}\text { Mean Difference (IV, } \\
\text { Random, 95\% CI) }\end{array}$ & Not estimable \\
\hline $\begin{array}{l}24 \text { Change in night time } \\
\text { awakening ( number of } \\
\text { nights) at endpoint }\end{array}$ & 1 & & $\begin{array}{l}\text { Mean Difference (IV, } \\
\text { Fixed, 95\% CI) }\end{array}$ & Totals not selected \\
\hline $\begin{array}{l}\text { 24.1 Mean baseline } \\
\text { FEV1 } 61-79 \% \text { of } \\
\text { predicted }\end{array}$ & 1 & & $\begin{array}{l}\text { Mean Difference (IV, } \\
\text { Fixed, 95\% CI) }\end{array}$ & Not estimable \\
\hline
\end{tabular}




\begin{tabular}{|c|c|c|c|c|}
\hline $\begin{array}{l}\text { Outcome or subgroup } \\
\text { title }\end{array}$ & No. of studies & No. of participants & Statistical method & Effect size \\
\hline $\begin{array}{l}25 \% \text { nights with } \\
\text { awakening }\end{array}$ & 1 & & $\begin{array}{l}\text { Mean Difference (IV, } \\
\text { Fixed, 95\% CI) }\end{array}$ & Totals not selected \\
\hline $\begin{array}{l}25.1 \text { Mean Baseline } \\
\text { FEV1 >/= } 80 \% \text { of } \\
\text { predicted }\end{array}$ & & & $\begin{array}{l}\text { Mean Difference (IV, } \\
\text { Fixed, 95\% CI) }\end{array}$ & Not estimable \\
\hline $\begin{array}{l}25.2 \text { Mean baseline } \\
\text { FEV1 } 61-79 \% \text { of } \\
\text { predicted }\end{array}$ & 1 & & $\begin{array}{l}\text { Mean Difference (IV, } \\
\text { Fixed, 95\% CI) }\end{array}$ & Not estimable \\
\hline $\begin{array}{l}26 \text { Change in } \% \\
\text { symptom-free days at } \\
\text { endpoint }\end{array}$ & 3 & 833 & $\begin{array}{l}\text { Mean Difference (IV, } \\
\text { Random, 95\% CI) }\end{array}$ & $1.30[-3.12,5.71]$ \\
\hline $\begin{array}{l}26.1 \text { Mean baseline } \\
\text { FEV1 }>/=80 \% \text { of } \\
\text { predicted }\end{array}$ & 3 & 833 & $\begin{array}{l}\text { Mean Difference (IV, } \\
\text { Random, 95\% CI) }\end{array}$ & $1.30[-3.12,5.71]$ \\
\hline $\begin{array}{l}26.2 \text { Mean baseline } \\
\text { FEV1 } 61-79 \% \text { of } \\
\text { predicted }\end{array}$ & 0 & 0 & $\begin{array}{l}\text { Mean Difference (IV, } \\
\text { Random, 95\% CI) }\end{array}$ & Not estimable \\
\hline $27 \%$ symptom-free days & 3 & 392 & $\begin{array}{l}\text { Std. Mean Difference (IV, } \\
\text { Random, 95\% CI) }\end{array}$ & $0.07[-0.14,0.27]$ \\
\hline $\begin{array}{l}27.1 \text { Mean baseline } \\
\text { FEV1 } 61-79 \% \text { of } \\
\text { predicted }\end{array}$ & 1 & 286 & $\begin{array}{l}\text { Std. Mean Difference (IV, } \\
\text { Random, 95\% CI) }\end{array}$ & $0.12[-0.11,0.35]$ \\
\hline $\begin{array}{l}27.2 \text { Mean baseline } \\
\text { FEV1 }>/=80 \% \text { of } \\
\text { predicted }\end{array}$ & 0 & 0 & $\begin{array}{l}\text { Std. Mean Difference (IV, } \\
\text { Random, 95\% CI) }\end{array}$ & Not estimable \\
\hline $\begin{array}{l}27.3 \text { Unclear baseline } \\
\text { FEV1 }\end{array}$ & 2 & 106 & $\begin{array}{l}\text { Std. Mean Difference (IV, } \\
\text { Random, 95\% CI) }\end{array}$ & $-0.12[-0.56,0.31]$ \\
\hline $\begin{array}{l}28 \% \text { symptom-free } \\
\text { nights at } 52+/-4 \text { weeks }\end{array}$ & 1 & & $\begin{array}{l}\text { Mean Difference (IV, } \\
\text { Fixed, 95\% CI) }\end{array}$ & Totals not selected \\
\hline $\begin{array}{l}28.1 \text { Mean Baseline } \\
\text { FEV1 not reported }\end{array}$ & 1 & & $\begin{array}{l}\text { Mean Difference (IV, } \\
\text { Fixed, 95\% CI) }\end{array}$ & Not estimable \\
\hline $\begin{array}{l}29 \text { Quality of life (P- } \\
\text { AQLQ) }\end{array}$ & 10 & 2333 & $\begin{array}{l}\text { Mean Difference (IV, } \\
\text { Fixed, 95\% CI) }\end{array}$ & $0.03[-0.04,0.11]$ \\
\hline $\begin{array}{l}\text { 29.1 Mean baseline } \\
\text { FEV1 61-79\% of } \\
\text { predicted }\end{array}$ & 4 & 747 & $\begin{array}{l}\text { Mean Difference (IV, } \\
\text { Fixed, 95\% CI) }\end{array}$ & $-0.12[-0.28,0.03]$ \\
\hline $\begin{array}{l}29.2 \text { Mean baseline } \\
\text { FEV } 1>/=80 \% \text { of } \\
\text { predicted }\end{array}$ & 6 & 1586 & $\begin{array}{l}\text { Mean Difference (IV, } \\
\text { Fixed, 95\% CI) }\end{array}$ & $0.07[-0.01,0.16]$ \\
\hline $\begin{array}{l}29.3 \text { Unclear baseline } \\
\text { FEV1 }\end{array}$ & 0 & 0 & $\begin{array}{l}\text { Mean Difference (IV, } \\
\text { Fixed, 95\% CI) }\end{array}$ & Not estimable \\
\hline $\begin{array}{l}30 \text { Total \# adverse } \\
\text { events }\end{array}$ & 15 & 3284 & $\begin{array}{c}\text { Risk Ratio (M-H, Fixed, } \\
95 \% \text { CI) }\end{array}$ & $1.04[0.98,1.10]$ \\
\hline $\begin{array}{l}30.1 \text { Mean baseline } \\
\text { FEV1 }>/=80 \% \text { of } \\
\text { predicted }\end{array}$ & 7 & 1424 & $\begin{array}{c}\text { Risk Ratio (M-H, Fixed, } \\
95 \% \text { CI) }\end{array}$ & $1.09[1.01,1.19]$ \\
\hline $\begin{array}{l}30.2 \text { Mean baseline } \\
\text { FEV1 } 61-79 \% \text { of } \\
\text { predicted }\end{array}$ & 4 & 995 & $\begin{array}{c}\text { Risk Ratio (M-H, Fixed, } \\
95 \% \text { CI) }\end{array}$ & $1.02[0.93,1.12]$ \\
\hline $\begin{array}{l}\text { 30.3 Mean baseline } \\
\text { FEV1 not reported }\end{array}$ & 4 & 865 & $\begin{array}{c}\text { Risk Ratio (M-H, Fixed, } \\
95 \% \text { CI) }\end{array}$ & $0.94[0.80,1.09]$ \\
\hline $\begin{array}{l}31 \text { \# patients with } \\
\text { headache }\end{array}$ & 14 & 2966 & $\begin{array}{c}\text { Risk Ratio (M-H, Fixed, } \\
95 \% \text { CI) }\end{array}$ & $1.10[0.90,1.33]$ \\
\hline $\begin{array}{l}31.1 \text { Mean baseline } \\
\text { FEV1 }>/=80 \% \% \text { of } \\
\text { predicted }\end{array}$ & 4 & 779 & $\begin{array}{c}\text { Risk Ratio (M-H, Fixed, } \\
95 \% \text { CI) }\end{array}$ & $1.02[0.78,1.33]$ \\
\hline
\end{tabular}




\begin{tabular}{|c|c|c|c|c|}
\hline $\begin{array}{l}\text { Outcome or subgroup } \\
\text { title }\end{array}$ & No. of studies & No. of participants & Statistical method & Effect size \\
\hline $\begin{array}{l}\text { 31.2 Mean baseline } \\
\text { FEV1 } 61-79 \% \text { of } \\
\text { predicted }\end{array}$ & 7 & 1346 & $\begin{array}{c}\text { Risk Ratio (M-H, Fixed, } \\
95 \% \mathrm{CI})\end{array}$ & $1.18[0.85,1.65]$ \\
\hline $\begin{array}{l}\text { 31.3 Mean baseline } \\
\text { FEV1 not reported }\end{array}$ & 3 & 841 & $\begin{array}{c}\text { Risk Ratio (M-H, Fixed, } \\
95 \% \mathrm{CI})\end{array}$ & $1.15[0.65,2.04]$ \\
\hline $\begin{array}{l}32 \text { \# patients with oral } \\
\text { thrush }\end{array}$ & 5 & 1155 & $\begin{array}{c}\text { Risk Ratio (M-H, Fixed, } \\
95 \% \mathrm{CI})\end{array}$ & $3.78[0.63,22.75]$ \\
\hline $\begin{array}{l}\text { 32.1 Mean baseline } \\
\text { FEV1 } 61-79 \% \text { of } \\
\text { predicted }\end{array}$ & 1 & 206 & $\begin{array}{c}\text { Risk Ratio (M-H, Fixed, } \\
95 \% \mathrm{CI})\end{array}$ & $3.24[0.13,78.62]$ \\
\hline $\begin{array}{l}32.2 \mathrm{FEV} 1>/=80 \% \\
\text { predicted }\end{array}$ & 4 & 949 & $\begin{array}{c}\text { Risk Ratio (M-H, Fixed, } \\
95 \% \mathrm{CI})\end{array}$ & $4.04[0.46,35.52]$ \\
\hline $\begin{array}{l}\text { 32.3 Mean baseline } \\
\text { FEV1 not reported }\end{array}$ & 0 & 0 & $\begin{array}{c}\text { Risk Ratio (M-H, Fixed, } \\
95 \% \mathrm{CI})\end{array}$ & Not estimable \\
\hline 33 \# patients with tremor & 6 & 1467 & $\begin{array}{c}\text { Risk Ratio (M-H, Fixed, } \\
95 \% \mathrm{CI})\end{array}$ & $3.07[0.38,25.05]$ \\
\hline $\begin{array}{l}33.1 \text { Mean baseline } \\
\text { FEV } 1>/=80 \% \text { of } \\
\text { predicted }\end{array}$ & 3 & 959 & $\begin{array}{c}\text { Risk Ratio (M-H, Fixed, } \\
95 \% \mathrm{CI})\end{array}$ & $5.30[0.26,109.66]$ \\
\hline $\begin{array}{l}\text { 33.2 Mean baseline } \\
\text { FEV1 } 61-79 \% \text { of } \\
\text { predicted }\end{array}$ & 3 & 508 & $\begin{array}{c}\text { Risk Ratio (M-H, Fixed, } \\
95 \% \mathrm{CI})\end{array}$ & $1.46[0.06,35.18]$ \\
\hline $\begin{array}{l}\text { 33.3 Mean baseline } \\
\text { FEV1 not reported }\end{array}$ & 0 & 0 & $\begin{array}{c}\text { Risk Ratio (M-H, Fixed, } \\
95 \% \mathrm{CI})\end{array}$ & Not estimable \\
\hline $\begin{array}{l}34 \text { \# patients with } \\
\text { tachycardia or } \\
\text { palpitations }\end{array}$ & 5 & 1052 & $\begin{array}{c}\text { Risk Ratio (M-H, Fixed, } \\
95 \% \mathrm{CI})\end{array}$ & $0.40[0.05,3.25]$ \\
\hline $\begin{array}{l}34.1 \text { Mean baseline } \\
\text { FEV } 1>/=80 \% \text { of } \\
\text { predicted }\end{array}$ & 2 & 545 & $\begin{array}{c}\text { Risk Ratio (M-H, Fixed, } \\
95 \% \mathrm{CI})\end{array}$ & $0.33[0.01,8.02]$ \\
\hline $\begin{array}{l}\text { 34.2 Mean baseline } \\
\text { FEV1 } 61-79 \% \text { of } \\
\text { predicted }\end{array}$ & 3 & 507 & $\begin{array}{c}\text { Risk Ratio (M-H, Fixed, } \\
95 \% \mathrm{CI})\end{array}$ & $0.49[0.03,7.61]$ \\
\hline $\begin{array}{l}\text { 34.3 Mean baseline } \\
\text { FEV1 not reported }\end{array}$ & 0 & 0 & $\begin{array}{c}\text { Risk Ratio (M-H, Fixed, } \\
95 \% \mathrm{CI})\end{array}$ & Not estimable \\
\hline $\begin{array}{l}35 \text { \# patients with } \\
\text { adverse cardiovascular } \\
\text { events }\end{array}$ & 2 & 148 & $\begin{array}{c}\text { Risk Ratio (M-H, Fixed, } \\
95 \% \mathrm{CI})\end{array}$ & $0.31[0.01,7.49]$ \\
\hline $\begin{array}{l}35.1 \text { Mean baseline } \\
\text { FEV } 1>/=80 \% \text { of } \\
\text { predicted }\end{array}$ & 1 & 116 & $\begin{array}{c}\text { Risk Ratio (M-H, Fixed, } \\
95 \% \mathrm{CI})\end{array}$ & $0.31[0.01,7.49]$ \\
\hline $\begin{array}{l}\text { 35.2 Mean baseline } \\
\text { FEV1 } 61-79 \% \text { of } \\
\text { predicted }\end{array}$ & 1 & 32 & $\begin{array}{c}\text { Risk Ratio (M-H, Fixed, } \\
95 \% \mathrm{CI})\end{array}$ & Not estimable \\
\hline $\begin{array}{l}36 \text { Change in } \% \text { PC } 20 \text { at } \\
\text { endpoint }\end{array}$ & 1 & & $\begin{array}{l}\text { Mean Difference (IV, } \\
\text { Fixed, 95\% CI) }\end{array}$ & Totals not selected \\
\hline $\begin{array}{l}36.1 \text { Mean baseline } \\
\text { FEV1 }>/=80 \% \text { of } \\
\text { predicted }\end{array}$ & 1 & & $\begin{array}{l}\text { Mean Difference (IV, } \\
\text { Fixed, 95\% CI) }\end{array}$ & Not estimable \\
\hline $\begin{array}{l}37 \text { Change in height } \\
(\mathrm{cm}) \text { as SD scores at } 24 \\
+/-4 \text { weeks }\end{array}$ & 1 & & $\begin{array}{l}\text { Mean Difference (IV, } \\
\text { Fixed, 95\% CI) }\end{array}$ & Totals not selected \\
\hline $\begin{array}{l}37.1 \text { Mean baseline } \\
\text { FEV } 1>/=80 \% \text { of } \\
\text { predicted }\end{array}$ & 1 & & $\begin{array}{l}\text { Mean Difference (IV, } \\
\text { Fixed, 95\% CI) }\end{array}$ & Not estimable \\
\hline
\end{tabular}




\begin{tabular}{lcccc}
\hline $\begin{array}{l}\text { Outcome or subgroup } \\
\text { title }\end{array}$ & No. of studies & No. of participants & Statistical method & Effect size \\
\hline $\begin{array}{l}38 \text { Change in height at } \\
\text { one year }\end{array}$ & 1 & $\begin{array}{c}\text { Mean Difference (IV, } \\
\text { Fixed, 95\% CI) }\end{array}$ & Totals not selected \\
\hline
\end{tabular}

\section{Comparison 2}

\section{Long-acting beta2 agonist + ICS versus placebo + higher dose of ICS}

\begin{tabular}{|c|c|c|c|c|}
\hline $\begin{array}{l}\text { Outcome or subgroup } \\
\text { title }\end{array}$ & No. of studies & No. of participants & Statistical method & Effect size \\
\hline $\begin{array}{l}1 \text { \# patients with } \\
\text { exacerbations requiring } \\
\text { oral steroids }\end{array}$ & 2 & 441 & $\begin{array}{l}\text { Risk Ratio (M-H, Fixed, } \\
95 \% \mathrm{CI})\end{array}$ & $1.50[0.65,3.48]$ \\
\hline $\begin{array}{l}1.1 \text { Baseline FEV1 }>= \\
80 \% \text { predicted }\end{array}$ & 2 & 441 & $\begin{array}{c}\text { Risk Ratio (M-H, Fixed, } \\
95 \% \mathrm{CI})\end{array}$ & $1.50[0.65,3.48]$ \\
\hline $\begin{array}{l}\text { 1.2 Baseline FEV1 } \\
61-79 \% \text { predicted }\end{array}$ & 0 & 0 & $\begin{array}{c}\text { Risk Ratio (M-H, Fixed, } \\
95 \% \mathrm{CI})\end{array}$ & Not estimable \\
\hline $\begin{array}{l}2 \# \text { patients with } \\
\text { exacerbations requiring } \\
\text { hospitalisation }\end{array}$ & 4 & 1026 & $\begin{array}{c}\text { Risk Ratio (M-H, Fixed, } \\
95 \% \mathrm{CI})\end{array}$ & $2.21[0.74,6.64]$ \\
\hline $\begin{array}{l}2.1 \text { Baseline FEV1 }>= \\
80 \% \text { predicted }\end{array}$ & 2 & 441 & $\begin{array}{l}\text { Risk Ratio (M-H, Fixed, } \\
95 \% \mathrm{CI})\end{array}$ & $3.01[0.32,28.65]$ \\
\hline $\begin{array}{l}\text { 2.2 Baseline FEV1 } \\
61-79 \% \text { predicted }\end{array}$ & 1 & 225 & $\begin{array}{l}\text { Risk Ratio (M-H, Fixed, } \\
95 \% \mathrm{CI})\end{array}$ & $3.17[0.67,14.95]$ \\
\hline $\begin{array}{l}2.3 \text { Mean baseline } \\
\text { FEV1 not reported }\end{array}$ & 1 & 360 & $\begin{array}{c}\text { Risk Ratio (M-H, Fixed, } \\
95 \% \mathrm{CI})\end{array}$ & $0.33[0.01,8.13]$ \\
\hline 3 Serious adverse events & 5 & 1037 & $\begin{array}{l}\text { Risk Ratio (M-H, } \\
\text { Random, 95\% CI) }\end{array}$ & $1.45[0.63,3.33]$ \\
\hline $\begin{array}{l}3.1 \text { Baseline FEV1 > = } \\
80 \% \text { predicted }\end{array}$ & 2 & 423 & $\begin{array}{l}\text { Risk Ratio (M-H, } \\
\text { Random, 95\% CI) }\end{array}$ & $1.00[0.26,3.91]$ \\
\hline $\begin{array}{l}\text { 3.2 Baseline FEV1 } \\
61-79 \% \text { predicted }\end{array}$ & 1 & 223 & $\begin{array}{l}\text { Risk Ratio (M-H, } \\
\text { Random, 95\% CI) }\end{array}$ & $2.90[1.10,7.64]$ \\
\hline $\begin{array}{l}\text { 3.3 Mean baseline } \\
\text { FEV1 not reported }\end{array}$ & 2 & 391 & $\begin{array}{l}\text { Risk Ratio (M-H, } \\
\text { Random, 95\% CI) }\end{array}$ & $0.51[0.10,2.77]$ \\
\hline 4 Total \# withdrawals & 5 & 1026 & $\begin{array}{l}\text { Risk Ratio (M-H, Fixed, } \\
95 \% \mathrm{CI})\end{array}$ & $0.71[0.42,1.20]$ \\
\hline $\begin{array}{l}\text { 4.1 Baseline FEV1 }>= \\
80 \% \text { predicted }\end{array}$ & 1 & 120 & $\begin{array}{l}\text { Risk Ratio (M-H, Fixed, } \\
95 \% \mathrm{CI})\end{array}$ & $1.25[0.35,4.43]$ \\
\hline $\begin{array}{l}\text { 4.2 Baseline FEV1 } \\
61-79 \% \text { predicted }\end{array}$ & 1 & 223 & $\begin{array}{l}\text { Risk Ratio (M-H, Fixed, } \\
95 \% \mathrm{CI})\end{array}$ & $0.65[0.30,1.39]$ \\
\hline $\begin{array}{l}\text { 4.3 Mean baseline } \\
\text { FEV1 not reported }\end{array}$ & 3 & 683 & $\begin{array}{c}\text { Risk Ratio (M-H, Fixed, } \\
95 \% \mathrm{CI})\end{array}$ & $0.59[0.24,1.49]$ \\
\hline $\begin{array}{l}5 \text { \# withdrawals due to } \\
\text { adverse events }\end{array}$ & 2 & 343 & $\begin{array}{l}\text { Risk Ratio (M-H, Fixed, } \\
95 \% \mathrm{CI})\end{array}$ & $1.44[0.25,8.42]$ \\
\hline $\begin{array}{l}5.1 \text { Baseline FEV1 }>= \\
80 \% \text { predicted }\end{array}$ & 1 & 120 & $\begin{array}{l}\text { Risk Ratio (M-H, Fixed, } \\
95 \% \mathrm{CI})\end{array}$ & $2.0[0.19,21.47]$ \\
\hline $\begin{array}{l}\text { 5.2 Baseline FEV1 } \\
61-79 \% \text { predicted }\end{array}$ & 1 & 223 & $\begin{array}{l}\text { Risk Ratio (M-H, Fixed, } \\
95 \% \mathrm{CI})\end{array}$ & $0.91[0.06,14.30]$ \\
\hline $\begin{array}{l}5.3 \text { Baseline FEV1 <= } \\
60 \% \text { predicted }\end{array}$ & 0 & 0 & $\begin{array}{l}\text { Risk Ratio (M-H, Fixed, } \\
95 \% \mathrm{CI})\end{array}$ & Not estimable \\
\hline $\begin{array}{l}5.4 \text { Mean baseline } \\
\text { FEV1 not reported }\end{array}$ & 0 & 0 & $\begin{array}{l}\text { Risk Ratio (M-H, Fixed, } \\
95 \% \mathrm{CI})\end{array}$ & Not estimable \\
\hline
\end{tabular}




\begin{tabular}{|c|c|c|c|c|}
\hline $\begin{array}{l}\text { Outcome or subgroup } \\
\text { title }\end{array}$ & No. of studies & No. of participants & Statistical method & Effect size \\
\hline $\begin{array}{l}6 \# \text { withdrawals due to } \\
\text { poor asthma control or } \\
\text { exacerbation }\end{array}$ & 1 & & $\begin{array}{c}\text { Risk Ratio (M-H, Fixed, } \\
95 \% \mathrm{CI})\end{array}$ & Totals not selected \\
\hline $\begin{array}{l}6.1 \text { Baseline FEV1 >= } \\
80 \% \text { predicted }\end{array}$ & 1 & & $\begin{array}{c}\text { Risk Ratio (M-H, Fixed, } \\
95 \% \text { CI) }\end{array}$ & Not estimable \\
\hline $\begin{array}{l}\text { 6.2 Baseline FEV1 } \\
\text { 61-79\% predicted }\end{array}$ & 0 & & $\begin{array}{c}\text { Risk Ratio (M-H, Fixed, } \\
95 \% \text { CI) }\end{array}$ & Not estimable \\
\hline $\begin{array}{l}6.3 \text { Baseline FEV1 }<= \\
60 \% \text { predicted }\end{array}$ & 0 & & $\begin{array}{c}\text { Risk Ratio (M-H, Fixed, } \\
95 \% \text { CI) }\end{array}$ & Not estimable \\
\hline $\begin{array}{l}7 \text { Change in FEV1 at } \\
\text { endpoint }\end{array}$ & 2 & 526 & $\begin{array}{c}\text { Mean Difference (Fixed, } \\
95 \% \text { CI) }\end{array}$ & $0.01[-0.03,0.05]$ \\
\hline $\begin{array}{l}\text { 7.1 Baseline FEV1 >= } \\
80 \% \text { predicted }\end{array}$ & 1 & 303 & $\begin{array}{c}\text { Mean Difference (Fixed, } \\
95 \% \mathrm{CI} \text { ) }\end{array}$ & Not estimable \\
\hline $\begin{array}{l}\text { 7.2 Baseline FEV1 } \\
\text { 61-79\% predicted }\end{array}$ & 1 & 223 & $\begin{array}{c}\text { Mean Difference (Fixed, } \\
95 \% \text { CI) }\end{array}$ & $0.04[-0.06,0.14]$ \\
\hline $8 \mathrm{FEV} 1$ at endpoint & 1 & & $\begin{array}{l}\text { Mean Difference (IV, } \\
\text { Random, 95\% CI) }\end{array}$ & Totals not selected \\
\hline $\begin{array}{l}\text { 8.1 Baseline FEV1 >= } \\
80 \% \text { predicted }\end{array}$ & 0 & & $\begin{array}{l}\text { Mean Difference (IV, } \\
\text { Random, 95\% CI) }\end{array}$ & Not estimable \\
\hline $\begin{array}{l}\text { 8.2 Baseline FEV1 } \\
\text { 61-79\% predicted }\end{array}$ & 0 & & $\begin{array}{l}\text { Mean Difference (IV, } \\
\text { Random, 95\% CI) }\end{array}$ & Not estimable \\
\hline $\begin{array}{l}\text { 8.3 Mean Baseline } \\
\text { FEV1 not reported }\end{array}$ & 1 & & $\begin{array}{l}\text { Mean Difference (IV, } \\
\text { Random, 95\% CI) }\end{array}$ & Not estimable \\
\hline $\begin{array}{l}9 \text { Change in FEV1 } \\
\text { predicted at endpoint }\end{array}$ & 1 & & $\%$ (Random, 95\% CI) & Totals not selected \\
\hline $\begin{array}{l}9.1 \text { Baseline FEV1 }>= \\
80 \% \text { predicted }\end{array}$ & 1 & & $\%$ (Random, 95\% CI) & Not estimable \\
\hline $\begin{array}{l}\text { 9.2 Baseline FEV1 } \\
\text { 61-79\% predicted }\end{array}$ & 0 & & $\%$ (Random, $95 \% \mathrm{CI}$ ) & Not estimable \\
\hline $\begin{array}{l}10 \text { Change in morning } \\
\mathrm{PEF} \text { (L/min) at endpoint }\end{array}$ & 4 & 1002 & $\begin{array}{l}\text { Mean Difference (IV, } \\
\text { Random, 95\% CI) }\end{array}$ & $7.55[3.57,11.53]$ \\
\hline $\begin{array}{l}10.1 \text { Baseline FEV } 1>= \\
80 \% \text { predicted }\end{array}$ & 2 & 423 & $\begin{array}{l}\text { Mean Difference (IV, } \\
\text { Random, 95\% CI) }\end{array}$ & $7.75[2.48,13.02]$ \\
\hline $\begin{array}{l}\text { 10.2 Baseline FEV1 } \\
\text { 61-79\% predicted }\end{array}$ & 1 & 223 & $\begin{array}{l}\text { Mean Difference (IV, } \\
\text { Random, 95\% CI) }\end{array}$ & $9.0[-0.07,18.07]$ \\
\hline $\begin{array}{l}\text { 10.3 Mean Baseline } \\
\text { FEV1 not reported }\end{array}$ & 1 & 356 & $\begin{array}{l}\text { Mean Difference (IV, } \\
\text { Random, 95\% CI) }\end{array}$ & $5.90[-2.28,14.08]$ \\
\hline 11 Change in pm PEF & & 882 & $\begin{array}{c}\text { Mean Difference (Fixed, } \\
95 \% \text { CI) }\end{array}$ & $5.50[1.21,9.79]$ \\
\hline $\begin{array}{l}\text { 11.1 Baseline FEV1 } \\
\text { predicted }>=80 \%\end{array}$ & 1 & 303 & $\begin{array}{c}\text { Mean Difference (Fixed, } \\
95 \% \text { CI) }\end{array}$ & $5.4[-0.56,11.36]$ \\
\hline $\begin{array}{l}\text { 11.2 Baseline FEV1 } \\
\text { 61-79\% predicted }\end{array}$ & 1 & 223 & $\begin{array}{c}\text { Mean Difference (Fixed, } \\
95 \% \text { CI) }\end{array}$ & $7.0[-2.07,16.07]$ \\
\hline $\begin{array}{l}\text { 11.3 Mean Baseline } \\
\text { FEV1 not reported }\end{array}$ & 1 & 356 & $\begin{array}{c}\text { Mean Difference (Fixed, } \\
95 \% \mathrm{CI} \text { ) }\end{array}$ & $4.4[-4.05,12.85]$ \\
\hline $\begin{array}{l}12 \text { Change in clinic PEF } \\
(\mathrm{L} / \mathrm{min})\end{array}$ & 1 & & $\begin{array}{l}\text { Mean Difference (IV, } \\
\text { Fixed, 95\% CI) }\end{array}$ & Totals not selected \\
\hline $\begin{array}{l}\text { 12.1 Mean Baseline } \\
\text { FEV1 not reported }\end{array}$ & 1 & & $\begin{array}{l}\text { Mean Difference (IV, } \\
\text { Fixed, 95\% CI) }\end{array}$ & Not estimable \\
\hline $\begin{array}{l}13 \text { \# daytime rescue } \\
\text { inhalations at endpoint }\end{array}$ & 1 & & puffs/d (Fixed, 95\% CI) & Totals not selected \\
\hline $\begin{array}{l}\text { 13.1 Baseline FEV1 } \\
\text { 61-79\% predicted }\end{array}$ & 1 & & puffs/d (Fixed, 95\% CI) & Not estimable \\
\hline
\end{tabular}




\begin{tabular}{|c|c|c|c|c|}
\hline $\begin{array}{l}\text { Outcome or subgroup } \\
\text { title }\end{array}$ & No. of studies & No. of participants & Statistical method & Effect size \\
\hline $\begin{array}{l}14 \text { Number of nighttime } \\
\text { awakenings }\end{array}$ & 1 & & $\begin{array}{l}\text { Awakenings/yr (Fixed, } \\
95 \% \mathrm{CI} \text { ) }\end{array}$ & Totals not selected \\
\hline $\begin{array}{l}\text { 14.1 Baseline FEV1 } \\
\text { 61-79\% predicted }\end{array}$ & 1 & & $\begin{array}{c}\text { Awakenings/yr (Fixed, } \\
95 \% \mathrm{CI} \text { ) }\end{array}$ & Not estimable \\
\hline 15 Total \# adverse events & & 814 & $\begin{array}{l}\text { Risk Ratio (M-H, } \\
\text { Random, 95\% CI) }\end{array}$ & $1.05[0.90,1.23]$ \\
\hline $\begin{array}{l}15.1 \text { Baseline FEV1 }>= \\
80 \% \text { predicted }\end{array}$ & 1 & 120 & $\begin{array}{l}\text { Risk Ratio (M-H, } \\
\text { Random, } 95 \% \text { CI) }\end{array}$ & $1.13[1.02,1.26]$ \\
\hline $\begin{array}{l}\text { 15.2 Baseline FEV1 } \\
\text { 61-79\% predicted }\end{array}$ & 0 & 0 & $\begin{array}{l}\text { Risk Ratio (M-H, } \\
\text { Random, } 95 \% \text { CI) }\end{array}$ & Not estimable \\
\hline $\begin{array}{l}15.3 \text { Mean baseline } \\
\text { FEV1 not reported }\end{array}$ & 3 & 694 & $\begin{array}{l}\text { Risk Ratio (M-H, } \\
\text { Random, 95\% CI) }\end{array}$ & $0.99[0.84,1.16]$ \\
\hline $\begin{array}{l}16 \text { \# patients with } \\
\text { headache }\end{array}$ & 3 & 790 & $\begin{array}{c}\text { Risk Ratio (M-H, Fixed, } \\
95 \% \text { CI) }\end{array}$ & $1.37[0.98,1.90]$ \\
\hline $\begin{array}{l}16.1 \text { Baseline FEV1 >= } \\
80 \% \text { predicted }\end{array}$ & 1 & 120 & $\begin{array}{c}\text { Risk Ratio (M-H, Fixed, } \\
95 \% \mathrm{CI})\end{array}$ & $1.56[0.93,2.62]$ \\
\hline $\begin{array}{l}\text { 16.2 Baseline FEV1 } \\
61-79 \% \text { predicted }\end{array}$ & 0 & 0 & $\begin{array}{c}\text { Risk Ratio (M-H, Fixed, } \\
95 \% \mathrm{CI})\end{array}$ & Not estimable \\
\hline $\begin{array}{l}\text { 16.3 Mean baseline } \\
\text { FEV1 not reported }\end{array}$ & 2 & 670 & $\begin{array}{c}\text { Risk Ratio (M-H, Fixed, } \\
95 \% \text { CI) }\end{array}$ & $1.27[0.83,1.95]$ \\
\hline 17 Linear growth & 2 & & $\begin{array}{c}\text { Mean Difference (Fixed, } \\
95 \% \text { CI) }\end{array}$ & $1.21[0.72,1.70]$ \\
\hline $\begin{array}{l}18 \text { \# nighttime rescue } \\
\text { inhalations (puffs per day; } \\
\text { mean over study period) }\end{array}$ & 1 & & $\begin{array}{l}\text { Mean Difference (IV, } \\
\text { Random, 95\% CI) }\end{array}$ & Totals not selected \\
\hline $\begin{array}{l}\text { 18.1 Mean baseline } \\
\text { FEV1 } 61-79 \% \text { of } \\
\text { predicted }\end{array}$ & 0 & & $\begin{array}{l}\text { Mean Difference (IV, } \\
\text { Random, 95\% CI) }\end{array}$ & Not estimable \\
\hline $\begin{array}{l}\text { 18.2 Mean Baseline } \\
\text { FEV1 not reported }\end{array}$ & 1 & & $\begin{array}{l}\text { Mean Difference (IV, } \\
\text { Random, 95\% CI) }\end{array}$ & Not estimable \\
\hline $\begin{array}{l}19 \text { \# daytime rescue } \\
\text { inhalations (puffs per day; } \\
\text { mean over study period) }\end{array}$ & 1 & & $\begin{array}{l}\text { Mean Difference (IV, } \\
\text { Random, 95\% CI) }\end{array}$ & Totals not selected \\
\hline $\begin{array}{l}\text { 19.1 Mean baseline } \\
\text { FEV1 } 61-79 \% \text { of } \\
\text { predicted }\end{array}$ & 0 & & $\begin{array}{l}\text { Mean Difference (IV, } \\
\text { Random, 95\% CI) }\end{array}$ & Not estimable \\
\hline $\begin{array}{l}\text { 19.2 Mean Baseline } \\
\text { FEV1 not reported }\end{array}$ & 1 & & $\begin{array}{l}\text { Mean Difference (IV, } \\
\text { Random, 95\% CI) }\end{array}$ & Not estimable \\
\hline 20 Deaths & 2 & 459 & $\begin{array}{l}\text { Risk Difference (M-H, } \\
\text { Fixed, } 95 \% \text { CI) }\end{array}$ & Not estimable \\
\hline $\begin{array}{l}20.1 \text { Baseline FEV1 }>= \\
80 \% \text { predicted }\end{array}$ & 0 & 0 & $\begin{array}{l}\text { Risk Difference (M-H, } \\
\text { Fixed, } 95 \% \mathrm{CI})\end{array}$ & Not estimable \\
\hline $\begin{array}{l}\text { 20.2 Baseline FEV1 } \\
\text { 61-79\% predicted }\end{array}$ & 0 & 0 & $\begin{array}{l}\text { Risk Difference (M-H, } \\
\text { Fixed, } 95 \% \mathrm{CI})\end{array}$ & Not estimable \\
\hline $\begin{array}{l}20.3 \text { Mean baseline } \\
\text { FEV1 not reported }\end{array}$ & 2 & 459 & $\begin{array}{l}\text { Risk Difference (M-H, } \\
\text { Fixed, 95\% CI) }\end{array}$ & Not estimable \\
\hline $\begin{array}{l}21 \text { Daytime asthma } \\
\text { symptom score (mean } \\
\text { over study period) }\end{array}$ & 1 & & $\begin{array}{l}\text { Std. Mean Difference (IV, } \\
\text { Fixed, 95\% CI) }\end{array}$ & Totals not selected \\
\hline $\begin{array}{l}21.1 \text { Mean baseline } \\
\text { FEV } 1>/=80 \% \text { of } \\
\text { predicted }\end{array}$ & 0 & & $\begin{array}{l}\text { Std. Mean Difference (IV, } \\
\text { Fixed, 95\% CI) }\end{array}$ & Not estimable \\
\hline $\begin{array}{l}\text { 21.2 Mean Baseline } \\
\text { FEV1 not reported }\end{array}$ & 1 & & $\begin{array}{l}\text { Std. Mean Difference (IV, } \\
\text { Fixed, 95\% CI) }\end{array}$ & Not estimable \\
\hline
\end{tabular}




\begin{tabular}{|c|c|c|c|c|}
\hline $\begin{array}{l}\text { Outcome or subgroup } \\
\text { title }\end{array}$ & No. of studies & No. of participants & Statistical method & Effect size \\
\hline $\begin{array}{l}22 \text { Nighttime asthma } \\
\text { symptom score (mean } \\
\text { over study period) }\end{array}$ & 1 & & $\begin{array}{l}\text { Std. Mean Difference (IV, } \\
\text { Fixed, 95\% CI) }\end{array}$ & Totals not selected \\
\hline $\begin{array}{l}22.1 \text { Mean baseline } \\
\text { FEV } 1>/=80 \% \text { of } \\
\text { predicted }\end{array}$ & 0 & & $\begin{array}{l}\text { Std. Mean Difference (IV, } \\
\text { Fixed, 95\% CI) }\end{array}$ & Not estimable \\
\hline $\begin{array}{l}2.2 \text { Mean Baseline } \\
\text { FEV1 not reported }\end{array}$ & 1 & & $\begin{array}{l}\text { Std. Mean Difference (IV, } \\
\text { Fixed, 95\% CI) }\end{array}$ & Not estimable \\
\hline
\end{tabular}

Comparison 3

Subgroup analyses (comparison 01: LABA+ICS versus SAME DOSE ICS)

\begin{tabular}{|c|c|c|c|c|}
\hline Outcome or subgroup title & No. of studies & No. of participants & Statistical method & Effect size \\
\hline $\begin{array}{l}1 \text { \# patients with exacerbations } \\
\text { requiring oral steroids by FEV1 } \\
\% \text { predicted at baseline }\end{array}$ & 8 & 1084 & $\begin{array}{l}\text { Risk Ratio (M-H, } \\
\text { Fixed, 95\% CI) }\end{array}$ & $0.92[0.60,1.40]$ \\
\hline $\begin{array}{l}1.1 \text { Mean baseline FEV } 1>/= \\
80 \% \text { of predicted }\end{array}$ & 4 & 375 & $\begin{array}{l}\text { Risk Ratio (M-H, } \\
\text { Fixed, 95\% CI) }\end{array}$ & $0.87[0.47,1.64]$ \\
\hline $\begin{array}{l}\text { 1.2 Mean baseline FEV1 } \\
61-79 \% \text { of predicted }\end{array}$ & 2 & 230 & $\begin{array}{l}\text { Risk Ratio (M-H, } \\
\text { Fixed, 95\% CI) }\end{array}$ & $0.89[0.48,1.64]$ \\
\hline $\begin{array}{l}1.3 \text { Mean baseline FEV1 not } \\
\text { reported }\end{array}$ & 2 & 479 & $\begin{array}{l}\text { Risk Ratio (M-H, } \\
\text { Fixed, 95\% CI) }\end{array}$ & $1.54[0.26,9.09]$ \\
\hline $\begin{array}{l}2 \text { \# patients with exacerbations } \\
\text { requiring oral steroids by } \\
\text { whether funded by producers } \\
\text { ofLABA }\end{array}$ & 8 & 1084 & $\begin{array}{l}\text { Risk Ratio (M-H, } \\
\text { Fixed, 95\% CI) }\end{array}$ & $0.92[0.60,1.40]$ \\
\hline 2.1 Charity Funded & 1 & 23 & $\begin{array}{l}\text { Risk Ratio (M-H, } \\
\text { Fixed, 95\% CI) }\end{array}$ & $1.09[0.28,4.32]$ \\
\hline $\begin{array}{l}2.2 \text { Funded by manufacturers } \\
\text { of LABA }\end{array}$ & 7 & 1061 & $\begin{array}{l}\text { Risk Ratio (M-H, } \\
\text { Fixed, 95\% CI) }\end{array}$ & $0.90[0.58,1.41]$ \\
\hline $\begin{array}{l}3 \text { \# patients with exacerbations } \\
\text { requiring oral steroids by dose } \\
\text { of ICS in both groups }\end{array}$ & 8 & 1084 & $\begin{array}{l}\text { Risk Ratio (M-H, } \\
\text { Fixed, 95\% CI) }\end{array}$ & $0.92[0.60,1.40]$ \\
\hline $\begin{array}{l}\text { 3.1 Low dose of ICS }(<=400 \\
\mathrm{mcg} / \text { day of BDP-eq) }\end{array}$ & 5 & 831 & $\begin{array}{l}\text { Risk Ratio (M-H, } \\
\text { Fixed, 95\% CI) }\end{array}$ & $0.91[0.48,1.76]$ \\
\hline $\begin{array}{l}\text { 3.2 Moderate dose of ICS } \\
\text { (401-800 mcg/day of BDP-eq) }\end{array}$ & 2 & 230 & $\begin{array}{l}\text { Risk Ratio (M-H, } \\
\text { Fixed, 95\% CI) }\end{array}$ & $0.89[0.48,1.64]$ \\
\hline $\begin{array}{l}\text { 3.3 High dose of ICS (>800 } \\
\text { mcg/day of BDP-eq) }\end{array}$ & 0 & 0 & $\begin{array}{l}\text { Risk Ratio (M-H, } \\
\text { Fixed, 95\% CI) }\end{array}$ & Not estimable \\
\hline $\begin{array}{l}3.4 \text { Unspecified dose of ICS } \\
\text { or range of dose only } \\
\text { mentioned }\end{array}$ & 1 & 23 & $\begin{array}{l}\text { Risk Ratio (M-H, } \\
\text { Fixed, 95\% CI) }\end{array}$ & $1.09[0.28,4.32]$ \\
\hline $\begin{array}{l}4 \text { \# patients with exacerbations } \\
\text { requiring oral steroids by } \\
\text { combination inhaler or separate } \\
\text { inhaler for LABA }\end{array}$ & 8 & 1084 & $\begin{array}{l}\text { Risk Ratio (M-H, } \\
\text { Fixed, 95\% CI) }\end{array}$ & $0.92[0.60,1.40]$ \\
\hline 4.1 Combination inhaler & 3 & 682 & $\begin{array}{l}\text { Risk Ratio (M-H, } \\
\text { Fixed, 95\% CI) }\end{array}$ & $1.02[0.30,3.47]$ \\
\hline 4.2 Separate inhaler & 5 & 402 & $\begin{array}{l}\text { Risk Ratio (M-H, } \\
\text { Fixed, 95\% CI) }\end{array}$ & $0.90[0.57,1.42]$ \\
\hline 4.3 Not reported & 0 & 0 & $\begin{array}{l}\text { Risk Ratio (M-H, } \\
\text { Fixed, 95\% CI) }\end{array}$ & Not estimable \\
\hline
\end{tabular}




\begin{tabular}{|c|c|c|c|c|}
\hline Outcome or subgroup title & No. of studies & No. of participants & Statistical method & Effect size \\
\hline $\begin{array}{l}5 \text { \# patients with exacerbations } \\
\text { requiring oral steroids by } \\
\text { whether LABA dose is usual or } \\
\text { higher than usual }\end{array}$ & 8 & 1084 & $\begin{array}{l}\text { Risk Ratio (M-H, } \\
\text { Fixed, 95\% CI) }\end{array}$ & $0.92[0.60,1.40]$ \\
\hline 5.1 LABA at usual dose & 7 & 1061 & $\begin{array}{l}\text { Risk Ratio (M-H, } \\
\text { Fixed, 95\% CI) }\end{array}$ & $0.90[0.58,1.41]$ \\
\hline $\begin{array}{l}\text { 5.2 LABA at higher than } \\
\text { usual dose }\end{array}$ & 1 & 23 & $\begin{array}{l}\text { Risk Ratio (M-H, } \\
\text { Fixed, 95\% CI) }\end{array}$ & $1.09[0.28,4.32]$ \\
\hline $\begin{array}{l}6 \text { \# patients with exacerbations } \\
\text { requiring oral steroids by type } \\
\text { of LABA }\end{array}$ & 8 & 1084 & $\begin{array}{l}\text { Risk Ratio (M-H, } \\
\text { Fixed, 95\% CI) }\end{array}$ & $0.92[0.60,1.40]$ \\
\hline 6.1 Formoterol & 1 & 32 & $\begin{array}{l}\text { Risk Ratio (M-H, } \\
\text { Fixed, 95\% CI) }\end{array}$ & Not estimable \\
\hline 6.2 Salmeterol & 7 & 1052 & $\begin{array}{l}\text { Risk Ratio (M-H, } \\
\text { Fixed, 95\% CI) }\end{array}$ & $0.92[0.60,1.40]$ \\
\hline $\begin{array}{l}7 \text { \# patients with exacerbations } \\
\text { requiring oral steroids by trial } \\
\text { duration }\end{array}$ & 8 & 1084 & $\begin{array}{l}\text { Risk Ratio (M-H, } \\
\text { Fixed, 95\% CI) }\end{array}$ & $0.92[0.60,1.40]$ \\
\hline $7.1<16$ weeks & 7 & 967 & $\begin{array}{l}\text { Risk Ratio (M-H, } \\
\text { Fixed, 95\% CI) }\end{array}$ & $0.90[0.55,1.49]$ \\
\hline $7.2>=24$ weeks & 1 & 117 & $\begin{array}{l}\text { Risk Ratio (M-H, } \\
\text { Fixed, 95\% CI) }\end{array}$ & $0.95[0.43,2.11]$ \\
\hline $\begin{array}{l}8 \text { Change in FEV1 at endpoint } \\
\text { (L or \% predicted) stratifying } \\
\text { by type of LABA used }\end{array}$ & 11 & & $\begin{array}{l}\text { Std. Mean Difference } \\
\quad \text { (Fixed, 95\% CI) }\end{array}$ & Subtotals only \\
\hline 8.1 Formoterol & 6 & & $\begin{array}{l}\text { Std. Mean Difference } \\
\quad \text { (Fixed, 95\% CI) }\end{array}$ & $0.34[0.23,0.45]$ \\
\hline 8.2 Salmeterol & 5 & & $\begin{array}{l}\text { Std. Mean Difference } \\
\quad \text { (Fixed, 95\% CI) }\end{array}$ & $0.30[0.12,0.48]$ \\
\hline
\end{tabular}

Comparison 4

Subgroup analyses (comparison 02: LABA+ICS versus HIGHER DOSE ICS)

\begin{tabular}{|c|c|c|c|c|}
\hline Outcome or subgroup title & No. of studies & No. of participants & Statistical method & Effect size \\
\hline $\begin{array}{l}1 \text { \# patients with exacerbations } \\
\text { requiring oral steroids by FEV1 } \\
\% \text { predicted at baseline }\end{array}$ & 1 & 120 & $\begin{array}{l}\text { Risk Ratio (M-H, } \\
\text { Fixed, } 95 \% \mathrm{CI})\end{array}$ & $1.43[0.58,3.50]$ \\
\hline $\begin{array}{l}1.1 \text { Baseline FEV1 > = } 80 \% \\
\text { predicted }\end{array}$ & 1 & 120 & $\begin{array}{l}\text { Risk Ratio (M-H, } \\
\text { Fixed, 95\% CI) }\end{array}$ & $1.43[0.58,3.50]$ \\
\hline $\begin{array}{l}\text { 1.2 Baseline FEV1 61-79\% } \\
\text { predicted }\end{array}$ & 0 & 0 & $\begin{array}{l}\text { Risk Ratio (M-H, } \\
\text { Fixed, 95\% CI) }\end{array}$ & Not estimable \\
\hline $\begin{array}{l}2 \text { \# patients with exacerbations } \\
\text { requiring oral steroids by } \\
\text { whether funded by producers } \\
\text { ofLABA }\end{array}$ & 1 & 120 & $\begin{array}{l}\text { Risk Ratio (M-H, } \\
\text { Fixed, } 95 \% \text { CI) }\end{array}$ & $1.43[0.58,3.50]$ \\
\hline 2.1 Charity Funded & 0 & 0 & $\begin{array}{l}\text { Risk Ratio (M-H, } \\
\text { Fixed, 95\% CI) }\end{array}$ & Not estimable \\
\hline $\begin{array}{l}2.2 \text { Funded by manufacturers } \\
\text { of LABA }\end{array}$ & 1 & 120 & $\begin{array}{l}\text { Risk Ratio (M-H, } \\
\text { Fixed, 95\% CI) }\end{array}$ & $1.43[0.58,3.50]$ \\
\hline $\begin{array}{l}3 \text { \# patients with exacerbations } \\
\text { requiring oral steroids by dose } \\
\text { of ICS in control groups }\end{array}$ & 1 & 120 & $\begin{array}{l}\text { Risk Ratio (M-H, } \\
\text { Fixed, 95\% CI) }\end{array}$ & $1.43[0.58,3.50]$ \\
\hline
\end{tabular}




\begin{tabular}{|c|c|c|c|c|}
\hline Outcome or subgroup title & No. of studies & No. of participants & Statistical method & Effect size \\
\hline $\begin{array}{l}\text { 3.1 Low dose of ICS }(<=400 \\
\mathrm{mcg} / \text { day of BDP-eq) }\end{array}$ & 1 & 120 & $\begin{array}{l}\text { Risk Ratio (M-H, } \\
\text { Fixed, 95\% CI) }\end{array}$ & $1.43[0.58,3.50]$ \\
\hline $\begin{array}{c}\text { 3.2 Moderate dose of ICS } \\
(401-800 \mathrm{mcg} / \text { day of BDP-eq) }\end{array}$ & 0 & 0 & $\begin{array}{l}\text { Risk Ratio (M-H, } \\
\text { Fixed, 95\% CI) }\end{array}$ & Not estimable \\
\hline $\begin{array}{l}\text { 3.3 High dose of ICS (>800 } \\
\text { mcg/day of BDP-eq) }\end{array}$ & 0 & 0 & $\begin{array}{l}\text { Risk Ratio (M-H, } \\
\text { Fixed, 95\% CI) }\end{array}$ & Not estimable \\
\hline $\begin{array}{l}3.4 \text { Unspecified dose of ICS } \\
\text { or range of dose only } \\
\text { mentioned }\end{array}$ & 0 & 0 & $\begin{array}{l}\text { Risk Ratio (M-H, } \\
\text { Fixed, 95\% CI) }\end{array}$ & Not estimable \\
\hline $\begin{array}{l}4 \text { \# patients with exacerbations } \\
\text { requiring oral steroids by } \\
\text { combination inhaler or separate } \\
\text { inhaler for LABA }\end{array}$ & 1 & 120 & $\begin{array}{l}\text { Risk Ratio (M-H, } \\
\text { Fixed, } 95 \% \mathrm{CI})\end{array}$ & $1.43[0.58,3.50]$ \\
\hline 4.1 Combination inhaler & 0 & 0 & $\begin{array}{l}\text { Risk Ratio (M-H, } \\
\text { Fixed, 95\% CI) }\end{array}$ & Not estimable \\
\hline 4.2 Separate inhaler & 1 & 120 & $\begin{array}{l}\text { Risk Ratio (M-H, } \\
\text { Fixed, 95\% CI) }\end{array}$ & $1.43[0.58,3.50]$ \\
\hline 4.3 Not reported & 0 & 0 & $\begin{array}{l}\text { Risk Ratio (M-H, } \\
\text { Fixed, 95\% CI) }\end{array}$ & Not estimable \\
\hline $\begin{array}{l}5 \text { \# patients with exacerbations } \\
\text { requiring oral steroids by } \\
\text { whether LABA dose is usual or } \\
\text { higher than usual }\end{array}$ & 1 & 120 & $\begin{array}{l}\text { Risk Ratio (M-H, } \\
\text { Fixed, } 95 \% \mathrm{CI})\end{array}$ & $1.43[0.58,3.50]$ \\
\hline 5.1 LABA at usual dose & 1 & 120 & $\begin{array}{l}\text { Risk Ratio (M-H, } \\
\text { Fixed, 95\% CI) }\end{array}$ & $1.43[0.58,3.50]$ \\
\hline $\begin{array}{l}\text { 5.2 LABA at higher than } \\
\text { usual dose }\end{array}$ & 0 & 0 & $\begin{array}{l}\text { Risk Ratio (M-H, } \\
\text { Fixed, 95\% CI) }\end{array}$ & Not estimable \\
\hline $\begin{array}{l}6 \text { \# patients with exacerbations } \\
\text { requiring oral steroids by type } \\
\text { of LABA }\end{array}$ & 1 & 120 & $\begin{array}{l}\text { Risk Ratio (M-H, } \\
\text { Fixed, 95\% CI) }\end{array}$ & $1.43[0.58,3.50]$ \\
\hline 6.1 Formoterol & 0 & 0 & $\begin{array}{l}\text { Risk Ratio (M-H, } \\
\text { Fixed, 95\% CI) }\end{array}$ & Not estimable \\
\hline 6.2 Salmeterol & 1 & 120 & $\begin{array}{l}\text { Risk Ratio (M-H, } \\
\text { Fixed, 95\% CI) }\end{array}$ & $1.43[0.58,3.50]$ \\
\hline $\begin{array}{l}7 \text { \# patients with exacerbations } \\
\text { requiring oral steroids by trial } \\
\text { duration }\end{array}$ & 1 & 120 & $\begin{array}{l}\text { Risk Ratio (M-H, } \\
\text { Fixed, } 95 \% \mathrm{CI})\end{array}$ & $1.43[0.58,3.50]$ \\
\hline $7.1<16$ weeks & 0 & 0 & $\begin{array}{l}\text { Risk Ratio (M-H, } \\
\text { Fixed, 95\% CI) }\end{array}$ & Not estimable \\
\hline $7.2>=24$ weeks & 1 & 120 & $\begin{array}{l}\text { Risk Ratio (M-H, } \\
\text { Fixed, 95\% CI) }\end{array}$ & $1.43[0.58,3.50]$ \\
\hline
\end{tabular}

\section{Comparison 5}

WMD archive

\begin{tabular}{|c|c|c|c|c|}
\hline $\begin{array}{l}\text { Outcome or subgroup } \\
\text { title }\end{array}$ & No. of studies & No. of participants & Statistical method & Effect size \\
\hline $\begin{array}{l}1 \text { Change in FEV1 (\% } \\
\text { predicted) at endpoint }\end{array}$ & 4 & & $\begin{array}{l}\text { Mean Difference (IV, } \\
\text { Random, 95\% CI) }\end{array}$ & Totals not selected \\
\hline $\begin{array}{l}1.1 \text { Baseline FEV1 >= } \\
80 \% \text { predicted }\end{array}$ & 2 & & $\begin{array}{l}\text { Mean Difference (IV, } \\
\text { Random, 95\% CI) }\end{array}$ & Not estimable \\
\hline $\begin{array}{l}\text { 1.2 Baseline FEV1 } \\
61-79 \% \text { predicted }\end{array}$ & 2 & & $\begin{array}{l}\text { Mean Difference (IV, } \\
\text { Random, 95\% CI) }\end{array}$ & Not estimable \\
\hline
\end{tabular}




\begin{tabular}{|c|c|c|c|c|}
\hline $\begin{array}{l}\text { Outcome or subgroup } \\
\text { title }\end{array}$ & No. of studies & No. of participants & Statistical method & Effect size \\
\hline $\begin{array}{l}2 \text { Change in height at } 1 \\
\text { year }\end{array}$ & 1 & & $\begin{array}{l}\text { Mean Difference (IV, } \\
\text { Fixed, 95\% CI) }\end{array}$ & Totals not selected \\
\hline 3 Change in am PEF & 5 & & $\begin{array}{l}\text { Mean Difference (IV, } \\
\text { Fixed, 95\% CI) }\end{array}$ & Totals not selected \\
\hline $\begin{array}{l}4 \text { Change in FEV1 ( } \mathrm{L} \text { or } \\
\% \text { pred )stratifying on trial } \\
\text { duration }\end{array}$ & 4 & & $\begin{array}{l}\text { Std. Mean Difference (IV, } \\
\text { Random, 95\% CI) }\end{array}$ & Totals not selected \\
\hline $\begin{array}{l}\text { 4.1 Change in FEV1 }(\mathrm{L}) \\
\text { or }(\% \text { predicted) at } 6+/-2 \\
\text { weeks of treatment }\end{array}$ & 1 & & $\begin{array}{l}\text { Std. Mean Difference (IV, } \\
\text { Random, 95\% CI) }\end{array}$ & Not estimable \\
\hline $\begin{array}{l}4.2 \text { Change in FEV1 }(\mathrm{L}) \\
\text { or }(\% \text { predicted) at } 12+/- \\
4 \text { weeks of treatment }\end{array}$ & 4 & & $\begin{array}{l}\text { Std. Mean Difference (IV, } \\
\text { Random, 95\% CI) }\end{array}$ & Not estimable \\
\hline $\begin{array}{l}4.3 \text { Change in FEV1 }(\mathrm{L}) \\
\text { or }(\% \text { predicted) at } 24+/- \\
4 \text { weeks of treatment }\end{array}$ & 1 & & $\begin{array}{l}\text { Std. Mean Difference (IV, } \\
\text { Random, 95\% CI) }\end{array}$ & Not estimable \\
\hline $\begin{array}{l}4.4 \text { Change in FEV1 }(\mathrm{L}) \\
\text { or }(\% \text { predicted) at } 52+/- \\
4 \text { weeks of treatment }\end{array}$ & 0 & & $\begin{array}{l}\text { Std. Mean Difference (IV, } \\
\text { Random, 95\% CI) }\end{array}$ & Not estimable \\
\hline 5 am PEF & 3 & & $\begin{array}{l}\text { Mean Difference (IV, } \\
\text { Fixed, 95\% CI) }\end{array}$ & Totals not selected \\
\hline $6 \mathrm{Pm}$ PEF & 2 & & $\begin{array}{l}\text { Mean Difference (IV, } \\
\text { Fixed, 95\% CI) }\end{array}$ & Totals not selected \\
\hline 7 Change in pm PEF & 2 & & $\begin{array}{l}\text { Mean Difference (IV, } \\
\text { Fixed, 95\% CI) }\end{array}$ & Totals not selected \\
\hline $\begin{array}{l}8 \text { Change in FEV1 }(\mathrm{L}) \\
\text { versus baseline }\end{array}$ & 3 & & $\begin{array}{l}\text { Mean Difference (IV, } \\
\text { Fixed, 95\% CI) }\end{array}$ & Totals not selected \\
\hline $\begin{array}{l}9 \text { Change in evening PEF } \\
\text { (L/min) at endpoint }\end{array}$ & 2 & & $\begin{array}{l}\text { Mean Difference (IV, } \\
\text { Fixed, 95\% CI) }\end{array}$ & Totals not selected \\
\hline $\begin{array}{l}9.1 \text { Baseline FEV1 >= } \\
80 \% \text { predicted }\end{array}$ & 0 & & $\begin{array}{l}\text { Mean Difference (IV, } \\
\text { Fixed, 95\% CI) }\end{array}$ & Not estimable \\
\hline $\begin{array}{l}\text { 9.2 Baseline FEV1 } \\
61-79 \% \text { predicted }\end{array}$ & 1 & & $\begin{array}{l}\text { Mean Difference (IV, } \\
\text { Fixed, 95\% CI) }\end{array}$ & Not estimable \\
\hline $\begin{array}{l}\text { 9.3 Mean Baseline } \\
\text { FEV1 not reported }\end{array}$ & 1 & & $\begin{array}{l}\text { Mean Difference (IV, } \\
\text { Fixed, 95\% CI) }\end{array}$ & Not estimable \\
\hline
\end{tabular}




\section{Analysis 1.1}

Comparison 1 Long-acting beta 2 versus placebo: both groups receiving similar dose ICS, Outcome 1 \# patients with exacerbations requiring systemic steroids

Review: Addition of long-acting beta-agonists to inhaled corticosteroids for chronic asthma in children

Comparison: 1 Long-acting beta2 versus placebo: both groups receiving similar dose ICS Outcome: 1 \# patients with exacerbations requiring systemic steroids

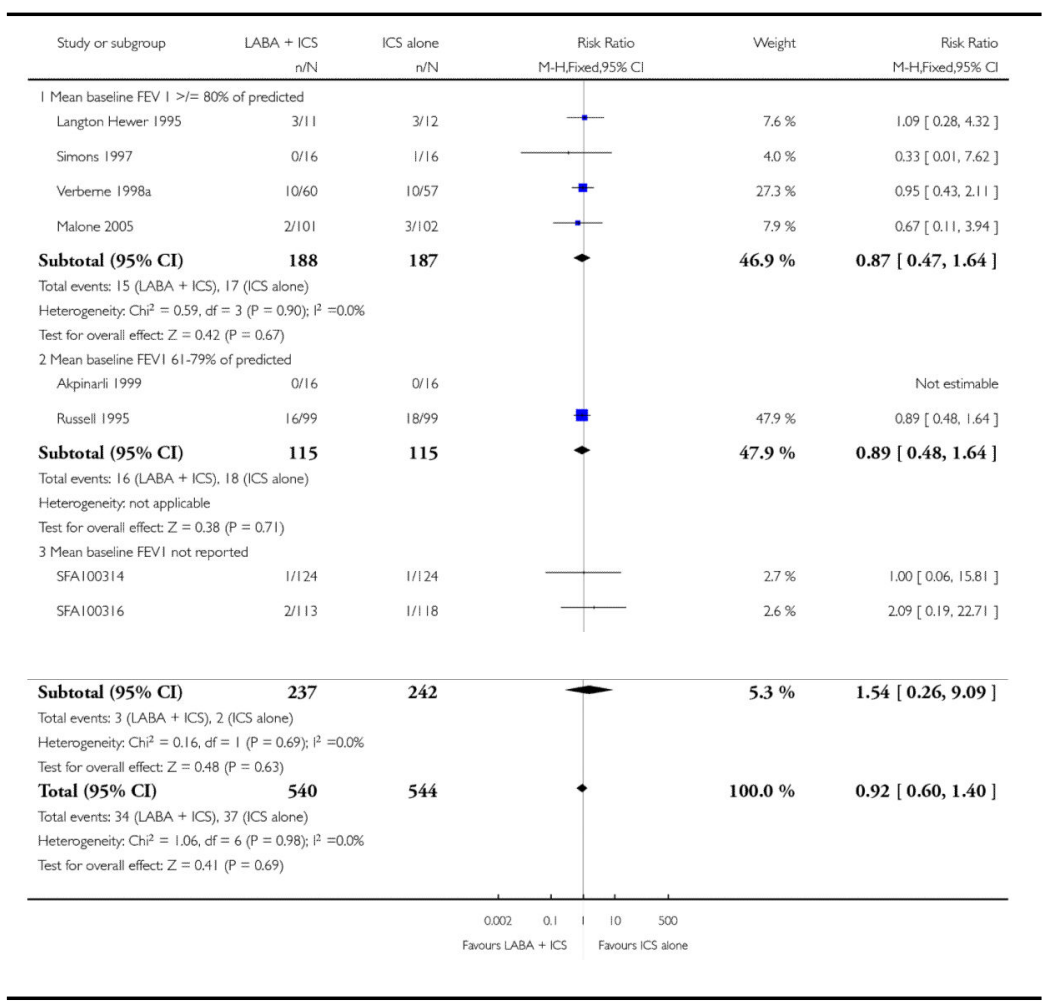




\section{Analysis 1.2}

Comparison 1 Long-acting beta 2 versus placebo: both groups receiving similar dose ICS, Outcome 2 \# patients with exacerbations requiring hospitalisation

Review: Addition of long-acting beta-agonists to inhaled corticosteroids for chronic asthma in children

Comparison: 1 Long-acting beta 2 versus placebo: both groups receiving similar dose ICS Outcome: 2 \# patients with exacerbations requiring hospitalisation

\begin{tabular}{|c|c|c|c|c|c|c|}
\hline Study or subgroup & $\begin{array}{r}\angle A B A+I C S \\
n / N \\
\end{array}$ & $\begin{array}{r}\text { ICS alone } \\
\mathrm{n} / \mathrm{N}\end{array}$ & \multicolumn{2}{|c|}{$\begin{array}{c}\text { Risk Ratio } \\
\text { M-H,Fixed,95\% CI }\end{array}$} & Weight & $\begin{array}{r}\text { Risk Ratio } \\
\text { M-H,Fixed,95\% CI }\end{array}$ \\
\hline \multicolumn{7}{|c|}{ | Mean baseline FEV $|>|=80 \%$ of predicted } \\
\hline Langton Hewer 1995 & $1 / 11$ & $0 / 12$ & & & $3.8 \%$ & $3.25[0.15,72.36]$ \\
\hline Verberne 1998a & 1/60 & $2 / 56$ & & - & $16.2 \%$ & $0.47[0.04,5.01]$ \\
\hline Subtotal $(95 \% \mathrm{CI})$ & 71 & 68 & & & $20.0 \%$ & $0.99[0.18,5.39]$ \\
\hline \multicolumn{7}{|c|}{ Total events: 2 (LABA + ICS), 2 (ICS alone) } \\
\hline \multicolumn{7}{|c|}{ Heterogeneity: $C h I^{2}=0.95$, df $=1(P=0.33): P^{2}=0.0 \%$} \\
\hline \multicolumn{7}{|c|}{ Test for overall effect $Z=0.01(P=0.99)$} \\
\hline \multicolumn{7}{|c|}{2 Mean baseline FEV I $61-79 \%$ of predicted } \\
\hline SD 0390714 & $4 / 136$ & 1/134 & & $\rightarrow$ & $7.9 \%$ & $3.94[0.45,34.80]$ \\
\hline Tal 2002 & $5 / 158$ & $0 / 138$ & & & $4.2 \%$ & $9.62[0.54,172.36]$ \\
\hline Russell 1995 & 9/99 & 9/107 & & 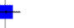 & $67.9 \%$ & $1.08[0.45,2.61]$ \\
\hline Subtotal $(95 \% \mathrm{CI})$ & 393 & 379 & & - & $80.0 \%$ & $1.81[0.86,3.82]$ \\
\hline \multicolumn{7}{|c|}{ Total events: 18 (LABA + ICS), 10 (ICS alone) } \\
\hline \multicolumn{7}{|c|}{ Heterogeneity: $C h^{2}=3.09$, df $=2(P=0.21): R^{2}=35 \%$} \\
\hline \multicolumn{7}{|c|}{ Test for overall effect: $Z=1.56(P=0.12)$} \\
\hline \multicolumn{7}{|c|}{3 Mean baseline FEVI not reported } \\
\hline SAM40012a & $0 / 180$ & $0 / 175$ & & & & Not estimable \\
\hline Subtotal $(95 \% \mathrm{CI})$ & 180 & 175 & & & & Not estimable \\
\hline \multicolumn{7}{|c|}{ Total events: 0 (LABA + ICS), 0 (ICS alone) } \\
\hline \multicolumn{7}{|c|}{ Heterogeneity, not applicable } \\
\hline \multicolumn{7}{|c|}{ Test for overall effect: not applicable } \\
\hline Total $(95 \%$ CI $)$ & 644 & 622 & & - & $100.0 \%$ & $1.65[0.83,3.25]$ \\
\hline \multicolumn{7}{|c|}{ Total everts: 20 (LABA + ICS), 12 (ICS alone) } \\
\hline \multicolumn{7}{|c|}{ Heterogencity $C \mathrm{Ch}^{2}=4.20, \mathrm{df}=4(\mathrm{P}=0.38) ; \mathrm{P}^{2}=5 \%$} \\
\hline \multicolumn{7}{|c|}{ Test for overall effect: $Z=1.44(P=0.15)$} \\
\hline & & & $0.005 \quad 0.1$ & 10 & & \\
\hline
\end{tabular}




\section{Analysis 1.3}

Comparison 1 Long-acting beta2 versus placebo: both groups receiving similar dose ICS, Outcome 3 Serious adverse events

Review: Addition of long-acting beta-agonists to inhaled corticosteroids for chronic asthma in children

Comparison: 1 Long-acting beta2 versus placebo: both groups receiving similar dose ICS Outcome: 3 Serious adverse events

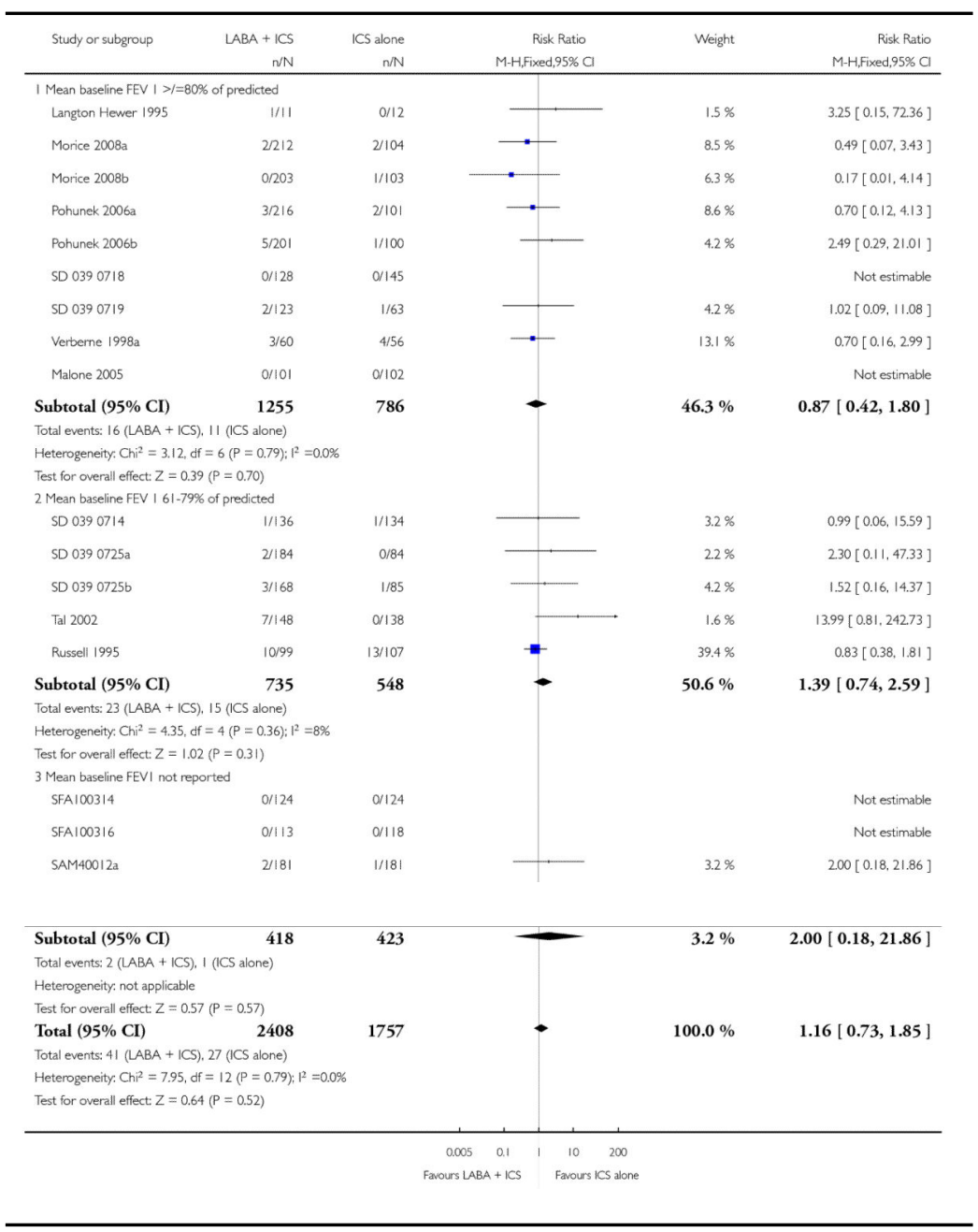




\section{Analysis 1.4}

Comparison 1 Long-acting beta 2 versus placebo: both groups receiving similar dose ICS, Outcome 4 Total \# withdrawals

Review: Addition of long-acting beta-agonists to inhaled corticosteroids for chronic asthma in children

Comparison: 1 Long-acting beta2 versus placebo: both groups receiving similar dose ICS Outcome: 4 Total \# withdrawals

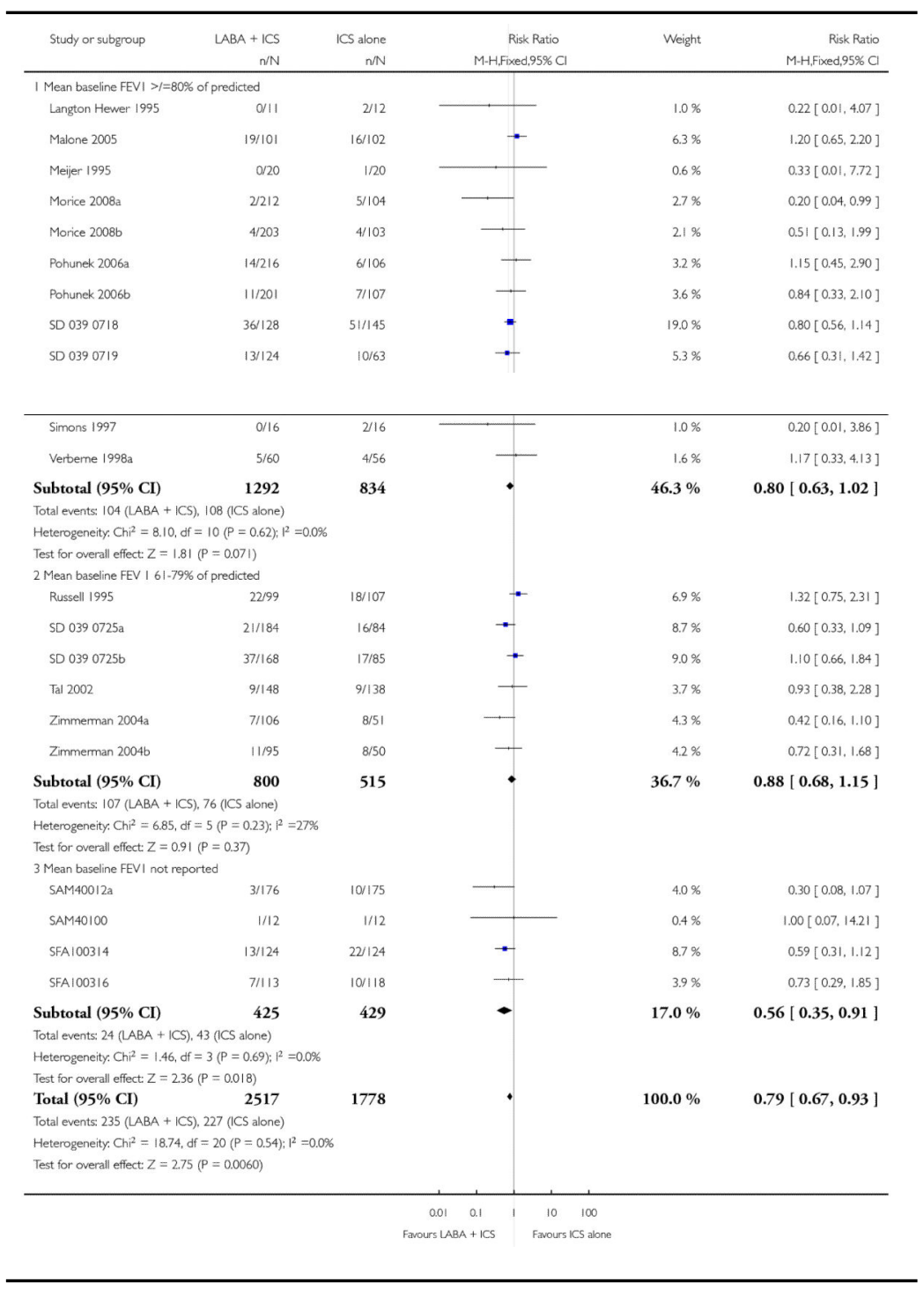




\section{Analysis 1.5}

Comparison 1 Long-acting beta 2 versus placebo: both groups receiving similar dose ICS, Outcome 5 \# withdrawals due to poor asthma control or exacerbation

Review: Addition of long-acting beta-agonists to inhaled corticosteroids for chronic asthma in children

Comparison: 1 Long-acting beta2 versus placebo: both groups receiving similar dose ICS Outcome: 5 \# withdrawals due to poor asthma control or exacerbation

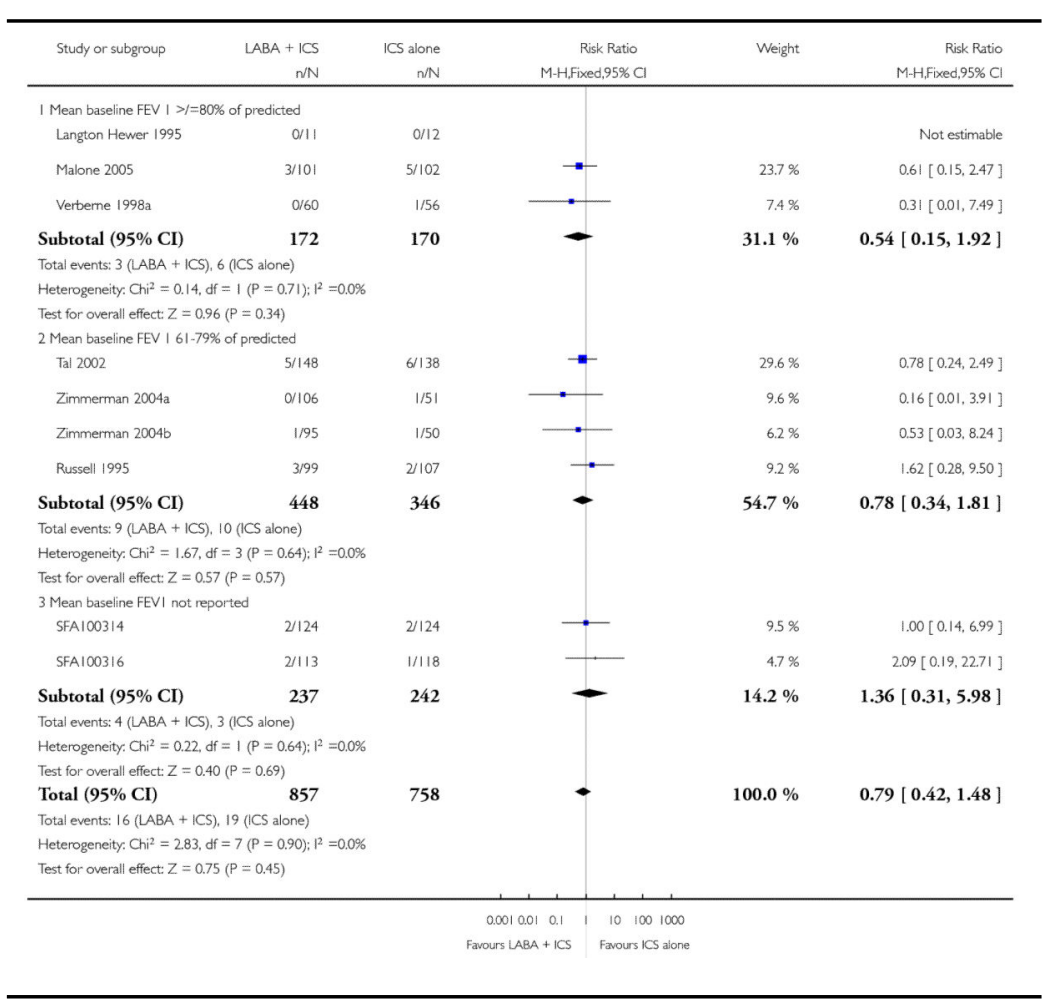




\section{Analysis 1.6}

Comparison 1 Long-acting beta2 versus placebo: both groups receiving similar dose ICS, Outcome 6 \# withdrawals due to serious non-respiratory event

Review: Addition of long-acting beta-agonists to inhaled corticosteroids for chronic asthma in children

Comparison: 1 Long-acting beta2 versus placebo: both groups receiving similar dose ICS Outcome: 6 \# withdrawals due to serious non-respiratory event

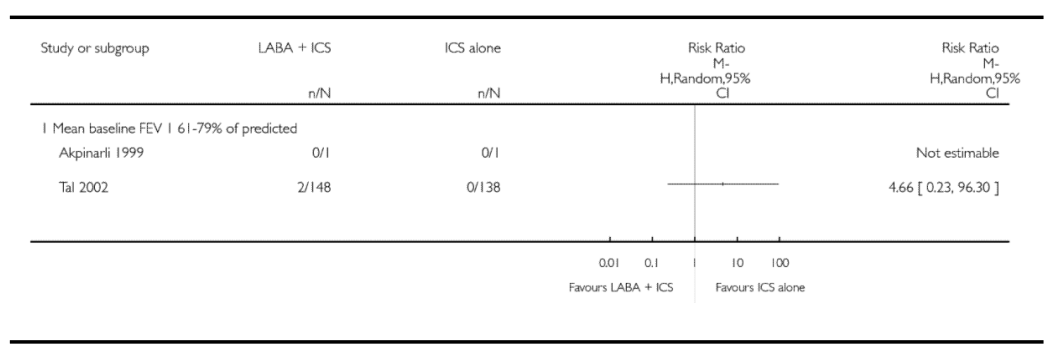




\section{Analysis 1.7}

Comparison 1 Long-acting beta 2 versus placebo: both groups receiving similar dose ICS, Outcome 7 \# withdrawals due to adverse events

Review: Addition of long-acting beta-agonists to inhaled corticosteroids for chronic asthma in children

Comparison: 1 Long-acting beta2 versus placebo: both groups receiving similar dose ICS Outcome: 7 \# withdrawals due to adverse events

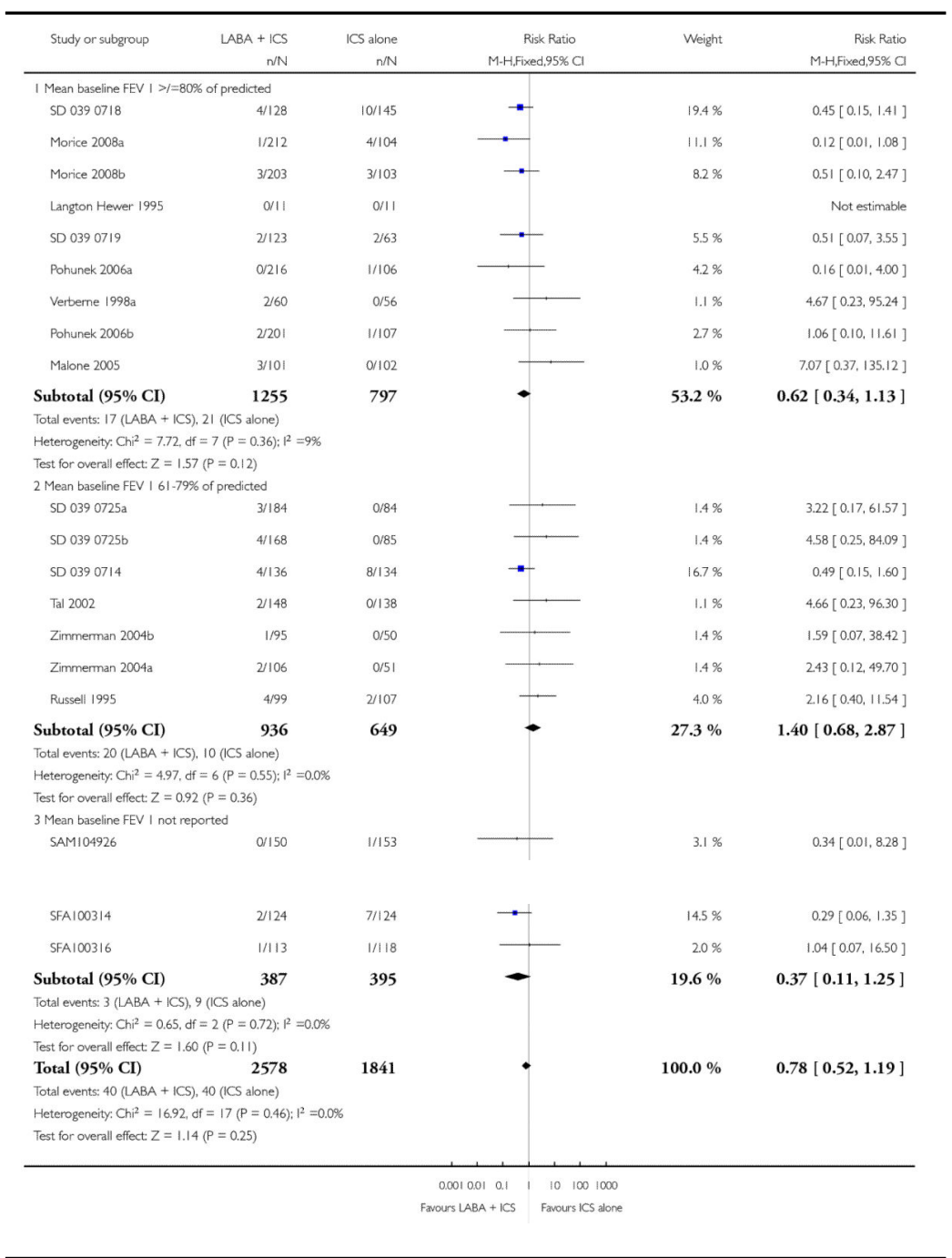




\section{Analysis 1.8}

Comparison 1 Long-acting beta 2 versus placebo: both groups receiving similar dose ICS, Outcome 8 Change in FEV1 at endpoint stratifying on baseline FEV1

Review: Addition of long-acting beta-agonists to inhaled corticosteroids for chronic asthma in children

Comparison: 1 Long-acting beta2 versus placebo: both groups receiving similar dose ICS Outcome: 8 Change in FEV1 at endpoint stratifying on baseline FEV1

\begin{tabular}{|c|c|c|c|c|c|c|c|}
\hline Study or subgroup & $\begin{array}{r}\angle A B A+I C S \\
N \\
\end{array}$ & $\begin{array}{r}\text { ICS alone } \\
\mathrm{N}\end{array}$ & Litres (SE) & IV.Rand & $\begin{array}{l}\text { Litres } \\
\text { tom, } 95 \% \mathrm{Cl}\end{array}$ & Weight & $\begin{array}{r}\text { Litres } \\
\text { IV.Random,95\% C }\end{array}$ \\
\hline \multicolumn{8}{|c|}{$\mid$ Mean Baseline FEVI $>\mid=80 \%$ of predicted } \\
\hline Langton Hewer 1995 & 11 & 10 & $0.42(0.107)$ & & $\longrightarrow$ & $1.8 \%$ & $0.42[0.21,0.63]$ \\
\hline Malone 2005 & 79 & 81 & $0.06(0.0408)$ & & - & $9.4 \%$ & $0.06[-0.02,0.14]$ \\
\hline Pohunek 20066 & 201 & 107 & $0.07(0.0306)$ & & $\rightarrow$ & $13.8 \%$ & $0.07[0.01,0.13]$ \\
\hline SD 0390719 & 0 & 0 & $0.08(0.03)$ & & $\rightarrow$ & $14.2 \%$ & $0.08[0.02,0.14]$ \\
\hline Pohunek 2006a & 213 & 106 & $0.07(0.0306)$ & & $\rightarrow$ & $13.8 \%$ & $0.07[0.01,0.13]$ \\
\hline $\begin{array}{l}\text { Subtotal }(\mathbf{9 5} \% \mathbf{C I}) \\
\text { Heterogeneity: } \text { Tau }^{2}=0.0 \\
\text { Test for overall effect: } Z=\end{array}$ & $\begin{array}{r}504 \\
-h^{2}=10.55, d f \\
4(P=0.0012)\end{array}$ & $\begin{array}{r}304 \\
(P=0.03) ;\end{array}$ & $62 \%$ & & - & $53.0 \%$ & $0.09[0.04,0.15]$ \\
\hline \multicolumn{8}{|c|}{2 Mean Baseline FEVI $61-79 \%$ of predicted } \\
\hline SD $0390725 \mathrm{a}$ & 0 & 0 & $0.07(0.0255)$ & & * & $17.0 \%$ & $0.07[0.02,0.12]$ \\
\hline SD 03907256 & 0 & 0 & $0.08(0.0255)$ & & * & $170 \%$ & $0.08[0.03,0.13]$ \\
\hline SD 0390714 & 133 & 131 & $0.13(0.045)$ & & $\rightarrow$ & $8.1 \%$ & $0.13[0.04,0.22]$ \\
\hline Russell 1995 & 76 & 87 & $0.09(0.061)$ & & - & $4.9 \%$ & $0.09[-0.03,0.21]$ \\
\hline \multicolumn{8}{|c|}{$\begin{array}{l}\text { Heterogeneity. Tat }{ }^{2}=0.0 ; \mathrm{Ch}^{2}=1.38, \mathrm{df}=3(\mathrm{P}=0.71) ; \mathrm{I}^{2}=0.0 \% \\
\text { Test for overall effect: } Z=5.15(P<0.00001)\end{array}$} \\
\hline $\begin{array}{l}\text { Subtotal }(95 \% \mathrm{CI}) \\
\text { Heterogeneity. not applica } \\
\text { Test for overall effect: not }\end{array}$ & $\begin{array}{l}0 \\
\text { licable }\end{array}$ & 0 & & & & & Not estimable \\
\hline \multicolumn{8}{|c|}{$\begin{array}{l}\text { Heterogeneity, Ta } \Psi^{2}=0.00 ; \mathrm{Ch}^{2}=11.96 \mathrm{df}=8(P=0.15): 1^{2}=33 \% \\
\text { Test for overall effect: } Z=5.78(P<0.00001)\end{array}$} \\
\hline & & & & $\begin{array}{l}-0.5 \quad-0.25 \\
\text { Favours treatment }\end{array}$ & $\begin{array}{ccc}0 & 0.25 & 0.5 \\
& \text { Favours control }\end{array}$ & & \\
\hline
\end{tabular}




\section{Analysis 1.9}

Comparison 1 Long-acting beta 2 versus placebo: both groups receiving similar dose ICS, Outcome 9 Change in FEV1 at endpoint (\% predicted) stratifying on baseline FEV1

Review: Addition of long-acting beta-agonists to inhaled corticosteroids for chronic asthma in children

Comparison: 1 Long-acting beta2 versus placebo: both groups receiving similar dose ICS Outcome: 9 Change in FEV1 at endpoint (\% predicted) stratifying on baseline FEV1

\begin{tabular}{|c|c|c|c|c|c|c|c|c|}
\hline \multirow[t]{2}{*}{ Study or subgroup } & \multirow{2}{*}{$\begin{array}{r}\angle A B A+I C S \\
N\end{array}$} & \multicolumn{3}{|c|}{ ICS alone } & \multirow{2}{*}{\multicolumn{2}{|c|}{$\begin{array}{c}\text { Mean } \\
\text { Difference } \\
\text { IV,Fixed,95\% Cl }\end{array}$}} & \multirow[t]{2}{*}{ Weight } & \multirow{2}{*}{$\begin{array}{r}\text { Mean } \\
\text { Difference } \\
\text { IV,Fixed, } 95 \% \mathrm{Cl}\end{array}$} \\
\hline & & $\operatorname{Mean}(S D)$ & $\mathrm{N}$ & $\operatorname{Mean}(S D)$ & & & & \\
\hline \multicolumn{9}{|c|}{ | Mean baseline FEV| >/= $80 \%$ predicted } \\
\hline Verberne 1998a & 60 & $4.36(10.53)$ & 57 & $1.28(9.13)$ & & \pm & $41.1 \%$ & $3.08[-0.49,6.65]$ \\
\hline Mejer 1995 & 20 & $5.8(11.6)$ & 19 & $2.2(9.15)$ & & $\cdot$ & $122 \%$ & $3.60[-2.94,10.14]$ \\
\hline Subtotal $(95 \% \mathrm{CI})$ & 80 & & 76 & & & - & $53.3 \%$ & $3.20[0.07,6.33]$ \\
\hline \multicolumn{9}{|c|}{ Heterogeneity: $C \mathrm{Ch}^{2}=0.02, \mathrm{df}=1(\mathrm{P}=0.89) ; \mathbf{1}^{2}=0.0 \%$} \\
\hline \multicolumn{9}{|c|}{ Test for overall effect: $Z=200(P=0.045)$} \\
\hline \multicolumn{9}{|c|}{2 Mean Baseline FEVI $61-79 \%$ of predicted } \\
\hline Akpinarli 1999 & 16 & $3(42.84)$ & 16 & $1(31.64)$ & & & $0.8 \%$ & $200[-24.10,28.10]$ \\
\hline Russell 1995 & 99 & $5.2(20)$ & 107 & $1.8(15.7)$ & & 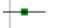 & $21.5 \%$ & $3.40[-1.54,8.34]$ \\
\hline Subtotal $(95 \% \mathrm{CI})$ & 115 & & 123 & & & - & $22.2 \%$ & $3.35[-1.50,8.20]$ \\
\hline \multicolumn{9}{|c|}{ Heterogeneity: $C \mathrm{Ch}^{2}=0.01, \mathrm{df}=1\langle\mathrm{P}=0.92) ; 1^{2}=0.0 \%$} \\
\hline \multicolumn{9}{|c|}{ Test for overall effect: $Z=1.35(P=0.18)$} \\
\hline \multicolumn{9}{|c|}{3 Mean Baseline FEVI not reported } \\
\hline Teper 2005 & 43 & $69(13)$ & 39 & $7.3(8)$ & & - & $24.4 \%$ & $-0.40[-5.03,4.23]$ \\
\hline Subtotal $(95 \% \mathrm{CI})$ & 43 & & 39 & & & - & $24.4 \%$ & $-0.40[-5.03,4.23]$ \\
\hline \multicolumn{9}{|c|}{ Heterogeneity not applicable } \\
\hline \multicolumn{9}{|c|}{ Test for overal effect: $Z=0.17(P=0.87)$} \\
\hline Total $(95 \% \mathrm{CI})$ & 238 & & 238 & & & - & $100.0 \%$ & $2.35[0.07,4.64]$ \\
\hline \multicolumn{9}{|c|}{ Heterogeneity: $C h^{2}=1.83, d f=4(P=0.77) ; 1^{2}=0.0 \%$} \\
\hline \multicolumn{9}{|c|}{ Test for overall effect: $Z=2.02(P=0.044)$} \\
\hline \multicolumn{9}{|c|}{ Test for subgroup differences: $C h i^{2}=1.80, d f=2(P=0.41), 1^{2}=0.0 \%$} \\
\hline & & & & & $-20 \quad-10$ & 10 & $\circ$ & \\
\hline
\end{tabular}




\section{Analysis 1.10}

Comparison 1 Long-acting beta2 versus placebo: both groups receiving similar dose ICS, Outcome 10 FEV1 predicted at endpoint stratifying on baseline FEV1

Review: Addition of long-acting beta-agonists to inhaled corticosteroids for chronic asthma in children

Comparison: 1 Long-acting beta2 versus placebo: both groups receiving similar dose ICS Outcome: 10 FEV1 predicted at endpoint stratifying on baseline FEV1

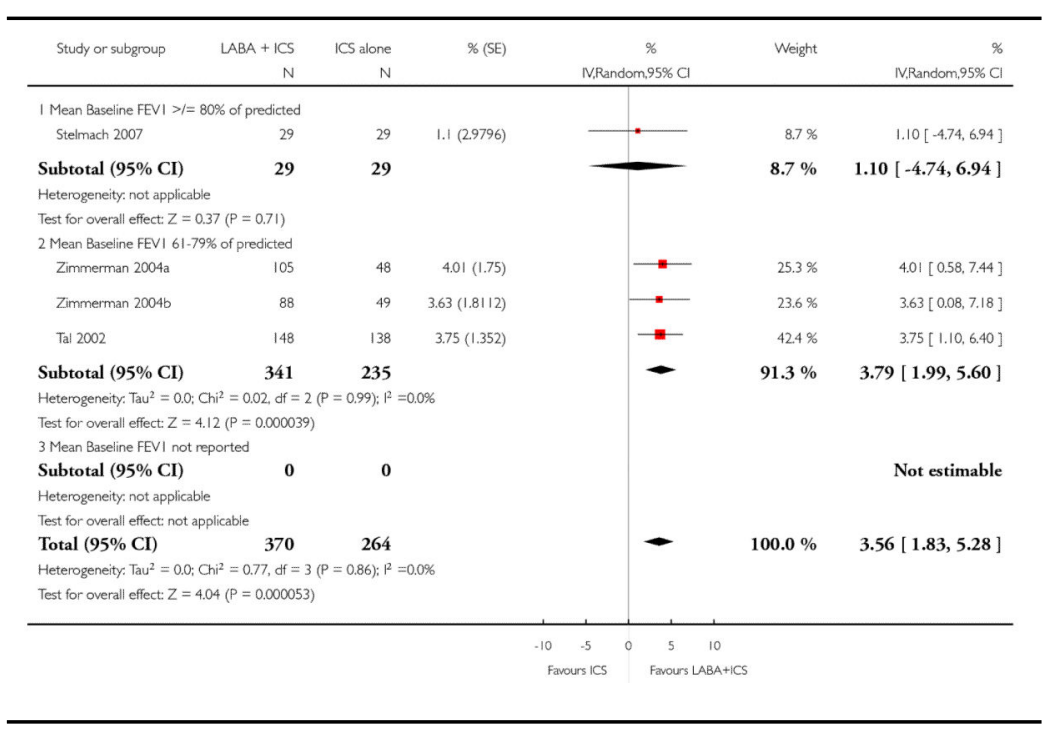




\section{Analysis 1.11}

Comparison 1 Long-acting beta2 versus placebo: both groups receiving similar dose ICS, Outcome 11 Change in FEV1 (L or \% pred) stratifying on trial duration

Review: Addition of long-acting beta-agonists to inhaled corticosteroids for chronic asthma in children

Comparison: 1 Long-acting beta2 versus placebo: both groups receiving similar dose ICS Outcome: 11 Change in FEV1 (L or \% pred) stratifying on trial duration

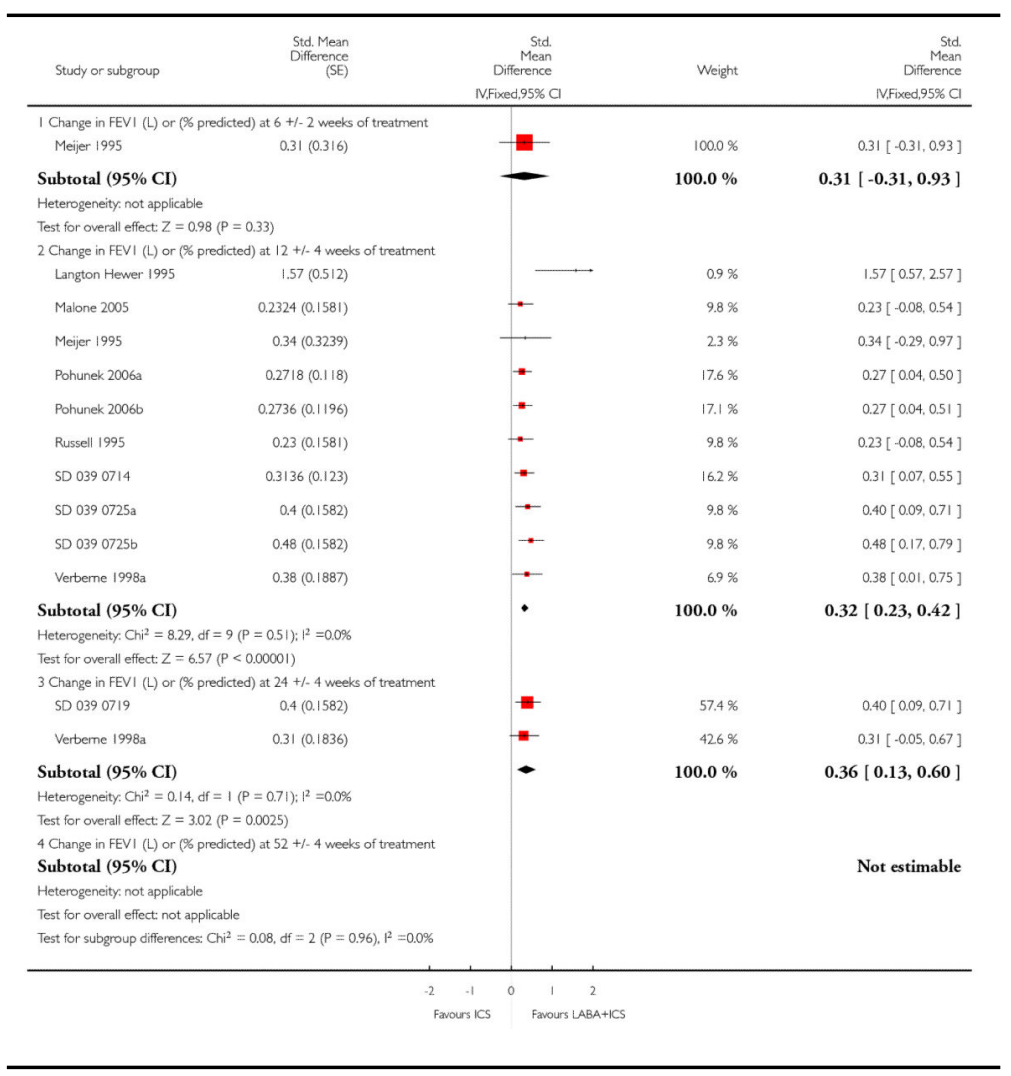




\section{Analysis 1.12}

Comparison 1 Long-acting beta2 versus placebo: both groups receiving similar dose ICS, Outcome 12 End of treatment FEV1 (L)

Review: Addition of long-acting beta-agonists to inhaled corticosteroids for chronic asthma in children

Comparison: 1 Long-acting beta2 versus placebo: both groups receiving similar dose ICS Outcome: 12 End of treatment FEV1 (L)

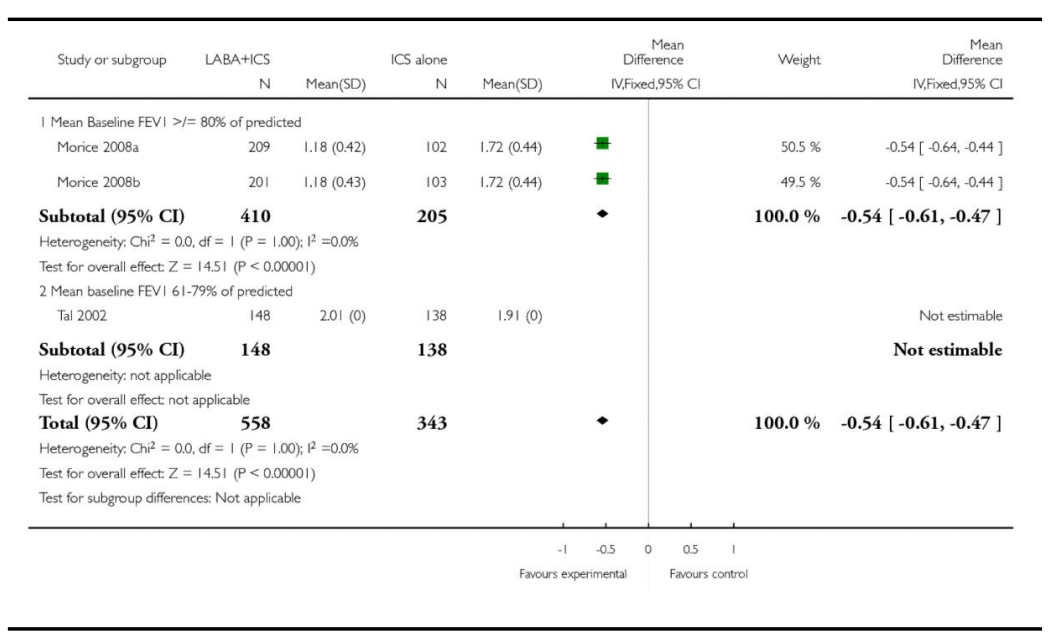

\section{Analysis 1.13}

Comparison 1 Long-acting beta2 versus placebo: both groups receiving similar dose ICS, Outcome 13 Change in PEF variability at endpoint

Review: Addition of long-acting beta-agonists to inhaled corticosteroids for chronic asthma in children

Comparison: 1 Long-acting beta2 versus placebo: both groups receiving similar dose ICS Outcome: 13 Change in PEF variability at endpoint

\begin{tabular}{|c|c|c|c|c|c|c|c|}
\hline Study or subgroup & $\begin{array}{r}\angle A B A+I C S \\
N\end{array}$ & \multicolumn{3}{|c|}{ ICS alone } & \multicolumn{2}{|c|}{$\begin{array}{c}\text { Mean } \\
\text { D.fference }\end{array}$} & $\begin{array}{r}\text { Mean } \\
\text { Difference } \\
\text { IV.Fixed,95\% Cl }\end{array}$ \\
\hline \multicolumn{8}{|c|}{ I Mean baseline FEV I $61-79 \%$ of presicted } \\
\hline \multirow[t]{2}{*}{ Russell |995 } & 75 & $-4.64(9.01)$ & 88 & $-3.35(7.15)$ & & & $-1.29[-3.82,1.24]$ \\
\hline & & & & & $\begin{array}{cc}-100 \quad-50 \\
\text { Favours LCS alone }\end{array}$ & $\begin{array}{l}50 \\
50 \\
\text { Farour: }\end{array}$ & \\
\hline
\end{tabular}




\section{Analysis 1.14}

Comparison 1 Long-acting beta 2 versus placebo: both groups receiving similar dose ICS, Outcome 14 Change in morning PEF at endpoint

Review: Addition of long-acting beta-agonists to inhaled corticosteroids for chronic asthma in children

Comparison: 1 Long-acting beta2 versus placebo: both groups receiving similar dose ICS Outcome: 14 Change in morning PEF at endpoint

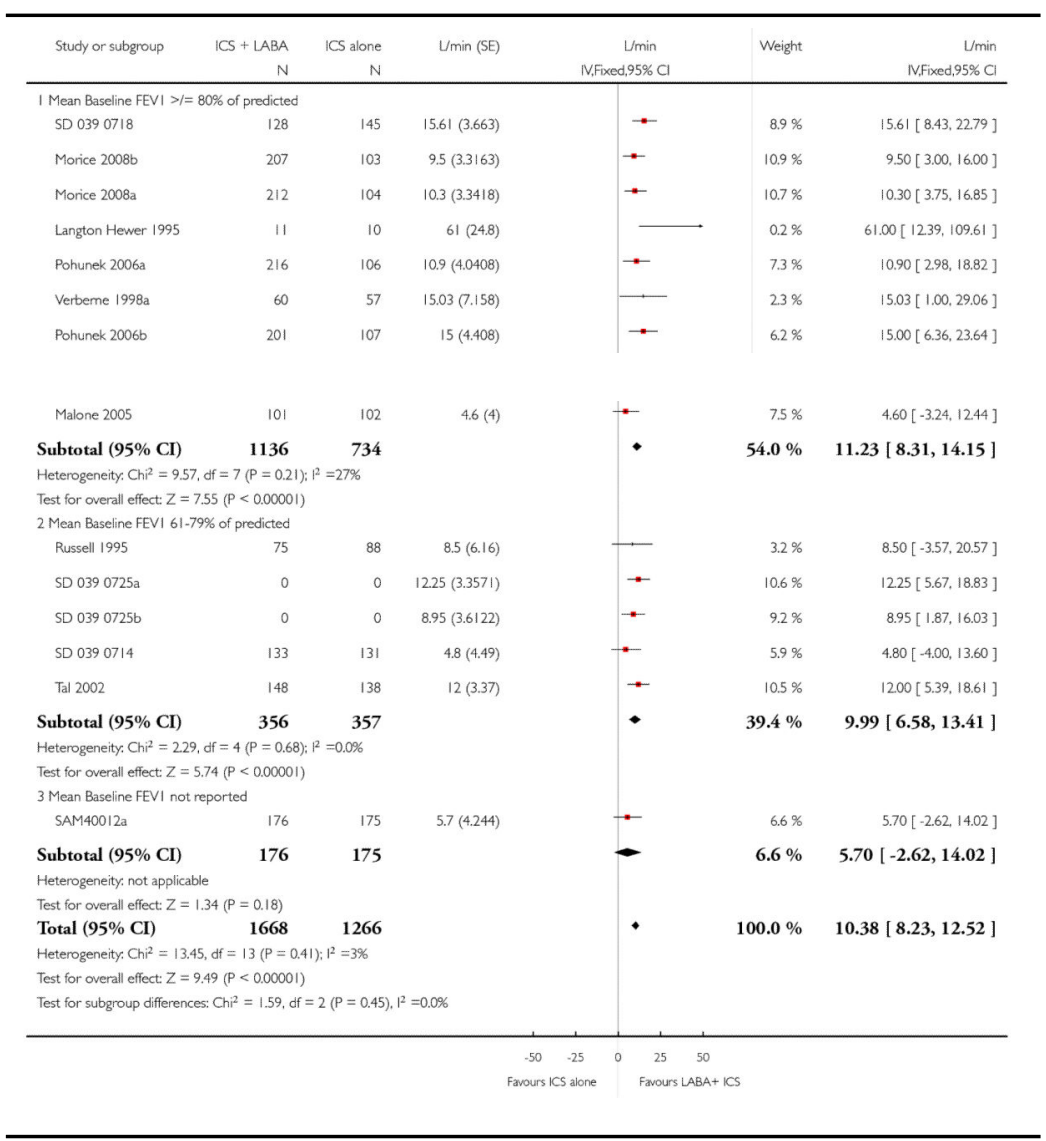




\section{Analysis 1.15}

Comparison 1 Long-acting beta2 versus placebo: both groups receiving similar dose ICS, Outcome 15 Morning PEF at endpoint

Review: Addition of long-acting beta-agonists to inhaled corticosteroids for chronic asthma in children

Comparison: 1 Long-acting beta2 versus placebo: both groups receiving similar dose ICS Outcome: 15 Morning PEF at endpoint

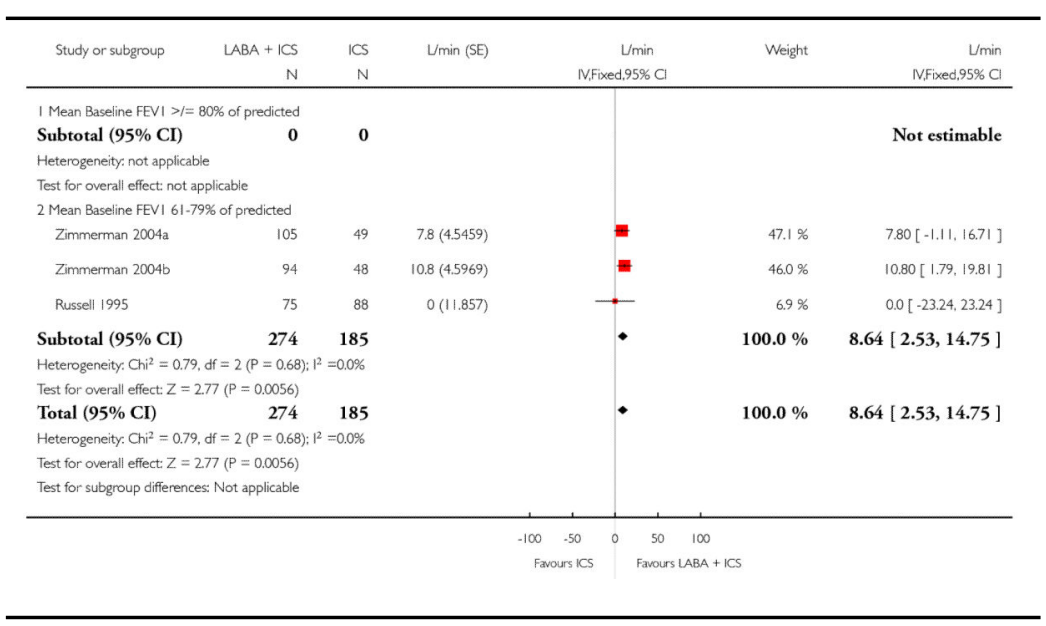

\section{Analysis 1.16}

Comparison 1 Long-acting beta2 versus placebo: both groups receiving similar dose ICS, Outcome 16 Change in morning PEF (\% predicted)

Review: Addition of long-acting beta-agonists to inhaled corticosteroids for chronic asthma in children

Comparison: 1 Long-acting beta2 versus placebo: both groups receiving similar dose ICS Outcome: 16 Change in morning PEF (\% predicted)

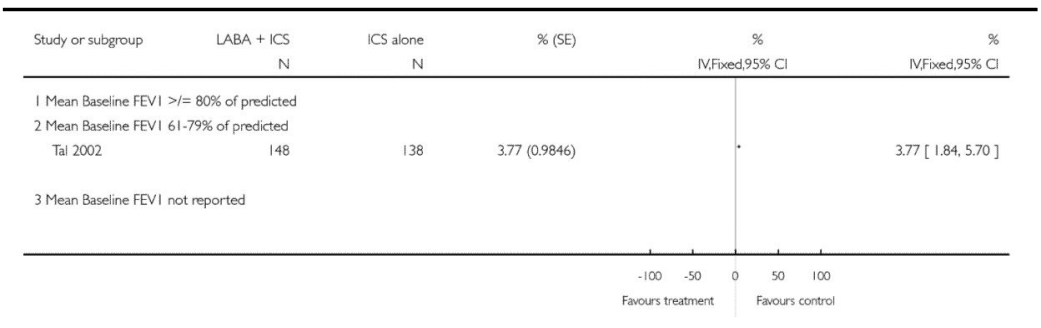




\section{Analysis 1.17}

Comparison 1 Long-acting beta2 versus placebo: both groups receiving similar dose ICS, Outcome 17 Change in evening PEF at endpoint

Review: Addition of long-acting beta-agonists to inhaled corticosteroids for chronic asthma in children

Comparison: 1 Long-acting beta2 versus placebo: both groups receiving similar dose ICS Outcome: 17 Change in evening PEF at endpoint

\begin{tabular}{|c|c|c|c|c|c|c|c|}
\hline Study or subgroup & $\begin{array}{r}\angle A B A+I C S \\
N \\
\end{array}$ & $\begin{array}{r}\text { ics alone } \\
\mathrm{N}\end{array}$ & Lmin (SE) & N,Fix & $\begin{array}{l}\text { Umin } \\
\mathrm{ed,9}, 9 \% \mathrm{Cl}\end{array}$ & Weight & $\begin{array}{r}U \text { min } \\
\text { IV,Fixed,95\% C }\end{array}$ \\
\hline \multicolumn{8}{|c|}{$\mid$ Mean Baseline FEV $>\mid=80 \%$ of predicted } \\
\hline Morice 2008a & 212 & 104 & $8.7(3.376)$ & & * & $13.3 \%$ & $8.70[208,15.32]$ \\
\hline Morice 2008b & 207 & 103 & $6.4(3.376)$ & & - & $13.3 \%$ & $6.40[-0.22,13.02]$ \\
\hline Langton Hewer 1995 & 11 & 10 & $90(25.6)$ & & 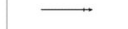 & $0.2 \%$ & $90.00[39.82,140.18]$ \\
\hline SD 0390718 & 0 & 0 & $|5.6|(4.6327)$ & & 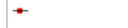 & $7.1 \%$ & $15.61[6.53,24.69]$ \\
\hline Pohunek 2006b & 201 & 107 & $12(4.3673)$ & & * & $7.9 \%$ & $1200[3.44,20.56]$ \\
\hline Malone 2005 & 101 & 102 & $6.4(3.69)$ & & * & $11.1 \%$ & $6.40[-0.83,13.63]$ \\
\hline Pohunek 2006a & 216 & 106 & $9.2(4.3673)$ & & - & $7.9 \%$ & $9.20[0.64,17.76]$ \\
\hline Subtotal $(95 \% \mathrm{CI})$ & 948 & 532 & & & - & $60.9 \%$ & $9.38[6.29,12.47]$ \\
\hline \multicolumn{8}{|c|}{ Heterogeneity: $\mathrm{Chi}^{2}=13.56, \mathrm{df}=6(P=0.03) ; 1^{2}=56 \%$} \\
\hline \multicolumn{3}{|c|}{2 Mean Baseline FEV| $61.79 \%$ of predicted } & \multicolumn{5}{|c|}{ Test for overall effect: $Z=5.95(P<0.00001)$} \\
\hline SD $0390725 a$ & 183 & 84 & $12.53(4.1888)$ & & - & $8.6 \%$ & $12.53[4.32,20.74]$ \\
\hline SD $0390725 b$ & 168 & 84 & $6.29(4.2296)$ & & - & $8.5 \%$ & $6.29[-200,14.58]$ \\
\hline Tal 2002 & 148 & 138 & $11.7(3.27)$ & & - & $14.2 \%$ & $11.70[5.29,18.11]$ \\
\hline Subtotal $(95 \% \mathrm{CI})$ & 499 & 306 & & & - & $31.3 \%$ & $10.46[6.15,14.78]$ \\
\hline \multicolumn{8}{|c|}{ Heterogeneity: $C \mathrm{Ch}^{2}=1.36, \mathrm{df}=2(\mathrm{P}=0.51) ; 1^{2}=0.0 \%$} \\
\hline \multicolumn{8}{|c|}{ Test for overal effect: $Z=4.75(P<0.00001)$} \\
\hline \multicolumn{8}{|c|}{3 Mean Baseline FEVI not reported } \\
\hline SAM40012a & 176 & 175 & $5(4.38)$ & & $\rightarrow$ & $7.9 \%$ & $5.00[-3.58,13.58]$ \\
\hline Subtotal $(95 \% \mathrm{CI})$ & 176 & 175 & & & - & $7.9 \%$ & $5.00[-3.58,13.58]$ \\
\hline \multicolumn{8}{|c|}{ Heterogeneity: not applicable } \\
\hline \multicolumn{8}{|c|}{ Test for overal effect: $Z=1.14(P=0.25)$} \\
\hline Total $(95 \% \mathrm{CI})$ & 1623 & 1013 & & & - & $100.0 \%$ & $9.37[6.96,11.79]$ \\
\hline \multirow{2}{*}{\multicolumn{8}{|c|}{$\begin{array}{l}\text { Heterogeneity, } \text { Chi }^{2}=16.16, \text { of }=10(P=0.10): 1^{2}=38 \% \\
\text { Test for overal effect: } Z=7.62(P<0.00001)\end{array}$}} \\
\hline \multicolumn{7}{|c|}{$\begin{array}{l}\text { Test for overall effect: } Z=7.62(P<0.00001) \\
\text { Test for subproup differences: } C h^{2}=1.24 \mathrm{df}=2(P=0.54), 1^{2}=0.0 \%\end{array}$} & \\
\hline \multicolumn{8}{|c|}{ Test for subgroup differences: $C h i^{2}=1.24, \mathrm{df}=2(P=0.54), 1^{2}=0.0 \%$} \\
\hline & & & & $\begin{array}{l}.100 \quad-50 \\
\text { Favours ics }\end{array}$ & $\begin{array}{ll}0 & 50 \quad 100 \\
\text { Faxours } L A B A+1 C S & \end{array}$ & & \\
\hline
\end{tabular}




\section{Analysis 1.18}

Comparison 1 Long-acting beta 2 versus placebo: both groups receiving similar dose ICS, Outcome 18 Change in evening PEF (\% predicted)

Review: Addition of long-acting beta-agonists to inhaled corticosteroids for chronic asthma in children

Comparison: 1 Long-acting beta2 versus placebo: both groups receiving similar dose ICS Outcome: 18 Change in evening PEF (\% predicted)

\begin{tabular}{|c|c|c|c|c|c|c|}
\hline Study or subgroup & $\begin{array}{r}\text { LABA }+ \text { ICS } \\
N\end{array}$ & $\begin{array}{r}\text { ICS alone } \\
\mathrm{N}\end{array}$ & $\%$ (SE) & \multicolumn{2}{|c|}{$\begin{array}{c}\% \\
\text { MFixed,95\% Cl }\end{array}$} & $\begin{array}{r}\% \\
\text { IV.Fixed,95\% C }\end{array}$ \\
\hline \multicolumn{7}{|c|}{$\mid$ Mean Baseline FEV $>>=80 \%$ of predicted } \\
\hline Tal 2002 & 148 & 138 & $3.4(0.9498)$ & & & $3.40[1.54,5.26]$ \\
\hline \multicolumn{7}{|c|}{3 Mean Baseline FEVI not reported } \\
\hline & & & & $\begin{array}{ccc}.100 & -50 \\
\text { Faycurs treatment }\end{array}$ & $\begin{array}{cc}50 \quad 100 \\
\text { Fawours controd }\end{array}$ & \\
\hline
\end{tabular}

Analysis 1.19

Comparison 1 Long-acting beta2 versus placebo: both groups receiving similar dose ICS, Outcome 19 Change in clinic PEF (L/min)

Review: Addition of long-acting beta-agonists to inhaled corticosteroids for chronic asthma in children

Comparison: 1 Long-acting beta2 versus placebo: both groups receiving similar dose ICS Outcome: 19 Change in clinic PEF (L/min)

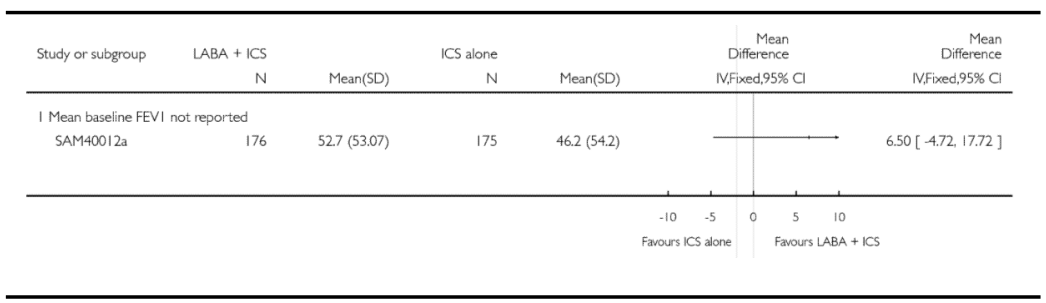




\section{Analysis 1.20}

Comparison 1 Long-acting beta 2 versus placebo: both groups receiving similar dose ICS, Outcome 20 Change in \# daytime rescue inhalations (puffs per day) at endpoint

Review: Addition of long-acting beta-agonists to inhaled corticosteroids for chronic asthma in children

Comparison: 1 Long-acting beta2 versus placebo: both groups receiving similar dose ICS Outcome: 20 Change in \# daytime rescue inhalations (puffs per day) at endpoint

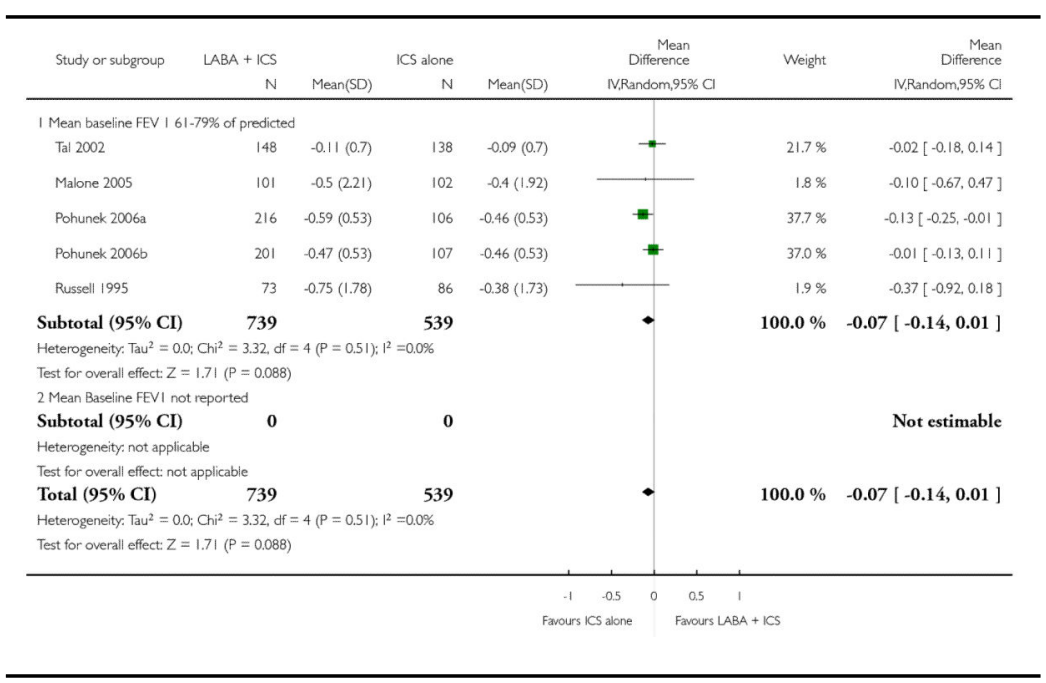




\section{Analysis 1.21}

Comparison 1 Long-acting beta2 versus placebo: both groups receiving similar dose ICS, Outcome $21 \%$ days without bronchodilator usage

Review: Addition of long-acting beta-agonists to inhaled corticosteroids for chronic asthma in children

Comparison: 1 Long-acting beta 2 versus placebo: both groups receiving similar dose ICS Outcome: $21 \%$ days without bronchodilator usage

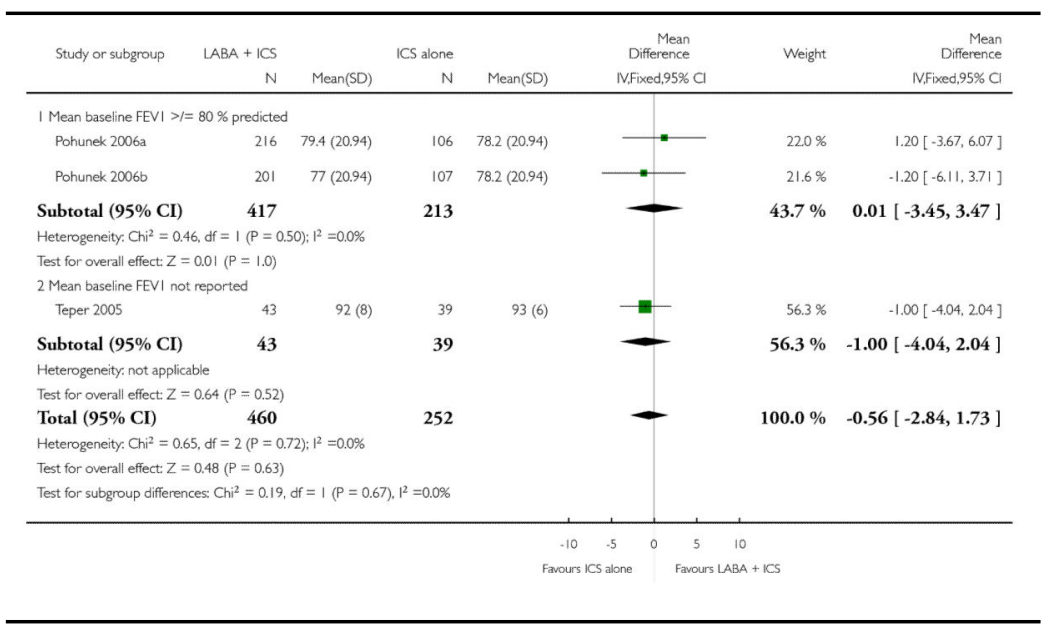

Analysis 1.22

Comparison 1 Long-acting beta2 versus placebo: both groups receiving similar dose ICS, Outcome 22 Mean change in asthma symptom score

Review: Addition of long-acting beta-agonists to inhaled corticosteroids for chronic asthma in children

Comparison: 1 Long-acting beta2 versus placebo: both groups receiving similar dose ICS Outcome: 22 Mean change in asthma symptom score

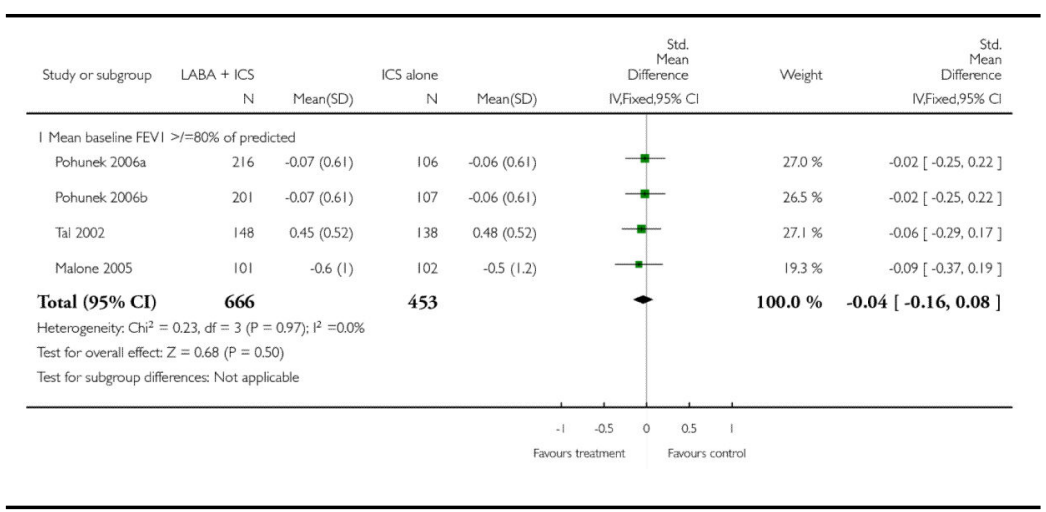




\section{Analysis 1.23}

Comparison 1 Long-acting beta2 versus placebo: both groups receiving similar dose ICS, Outcome 23 Change in \# nighttime rescue inhalations at endpoint

Review: Addition of long-acting beta-agonists to inhaled corticosteroids for chronic asthma in children

Comparison: 1 Long-acting beta2 versus placebo: both groups receiving similar dose ICS Outcome: 23 Change in \# nighttime rescue inhalations at endpoint

\begin{tabular}{|c|c|c|c|c|c|c|c|}
\hline Study or subgroup & $\begin{array}{r}\angle A B A+I C S \\
N\end{array}$ & \multicolumn{3}{|c|}{ ICS alone } & \multicolumn{2}{|c|}{$\begin{array}{c}\text { Mean } \\
\text { Difference } \\
\text { IV,Random,95\% CI }\end{array}$} & $\begin{array}{r}\text { Mean } \\
\text { Difference } \\
\text { |V.Random,95\% a }\end{array}$ \\
\hline \multicolumn{8}{|c|}{ I Mean baseline FEVI $61.79 \%$ of predicted } \\
\hline \multirow[t]{2}{*}{ Russell 1995} & 70 & $-0.15(1.05)$ & 83 & $-0.11(0.97)$ & 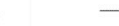 & - & $-0.04[-0.36,0.28]$ \\
\hline & & & & & $\begin{array}{c}-1 \quad 0.5 \\
\text { Favours iCS + }+ \text { LABA }\end{array}$ & $\begin{array}{c}0.5 \\
\text { Favours } 1\end{array}$ & \\
\hline
\end{tabular}

Analysis 1.24

Comparison 1 Long-acting beta2 versus placebo: both groups receiving similar dose ICS, Outcome 24 Change in night time awakening (number of nights) at endpoint

Review: Addition of long-acting beta-agonists to inhaled corticosteroids for chronic asthma in children

Comparison: 1 Long-acting beta2 versus placebo: both groups receiving similar dose ICS Outcome: 24 Change in night time awakening (number of nights) at endpoint

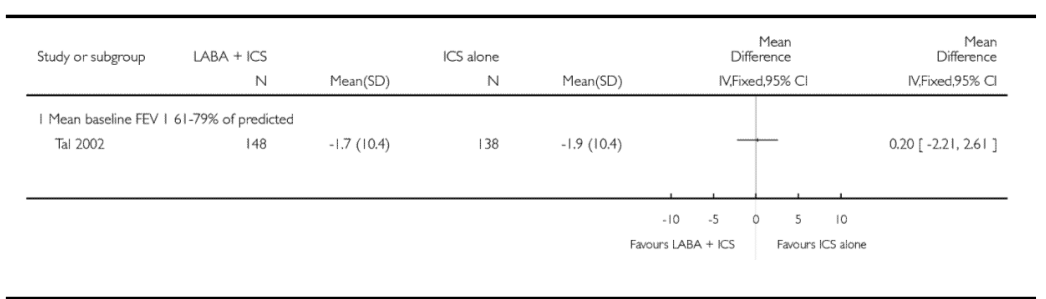




\section{Analysis 1.25}

Comparison 1 Long-acting beta 2 versus placebo: both groups receiving similar dose ICS, Outcome $25 \%$ nights with awakening

Review: Addition of long-acting beta-agonists to inhaled corticosteroids for chronic asthma in children

Comparison: 1 Long-acting beta2 versus placebo: both groups receiving similar dose ICS Outcome: $25 \%$ nights with awakening

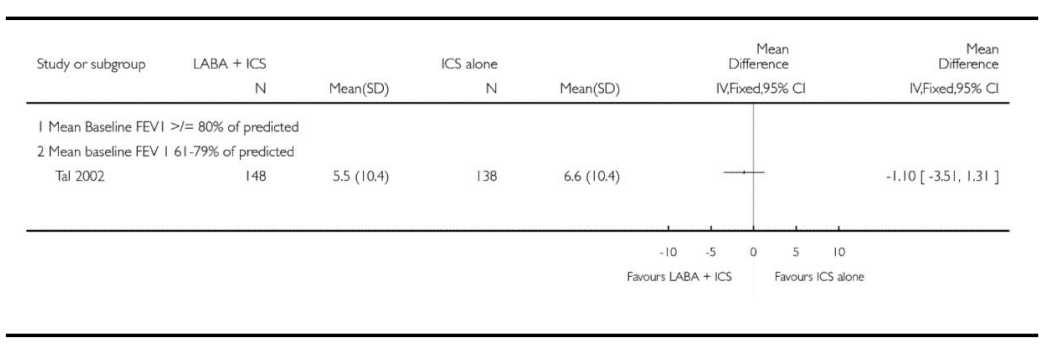

\section{Analysis 1.26}

Comparison 1 Long-acting beta2 versus placebo: both groups receiving similar dose ICS, Outcome 26 Change in \% symptom-free days at endpoint

Review: Addition of long-acting beta-agonists to inhaled corticosteroids for chronic asthma in children

Comparison: 1 Long-acting beta2 versus placebo: both groups receiving similar dose ICS Outcome: 26 Change in \% symptom-free days at endpoint

\begin{tabular}{|c|c|c|c|c|c|c|c|c|}
\hline \multirow[t]{2}{*}{ Study or subgroup } & \multirow{2}{*}{$\begin{array}{r}\mathrm{LABA}+\text { ICS } \\
\mathrm{N}\end{array}$} & \multicolumn{3}{|c|}{ ICS alone } & \multirow{2}{*}{\multicolumn{2}{|c|}{$\begin{array}{c}\text { Mean } \\
\text { Difference } \\
\text { IV.Random,95\% a }\end{array}$}} & \multirow[t]{2}{*}{ Weight } & \multirow{2}{*}{$\begin{array}{r}\text { Mean } \\
\text { Difference } \\
\text { IV.Random.95\% CI }\end{array}$} \\
\hline & & $\operatorname{Mean}(\mathrm{SD})$ & $\mathrm{N}$ & $\operatorname{Mean}(S D)$ & & & & \\
\hline \multicolumn{9}{|c|}{ | Mean baseline FEV $\mid>1=80 \%$ of predicted } \\
\hline Malone 2005 & 101 & $24.4(41)$ & 102 & $21.2(41)$ & & "- & $15.3 \%$ & $3.20[-8.08,14.48]$ \\
\hline Pohunek 2006a & 216 & $33(29.08)$ & 106 & $32(29.08)$ & & \# & $427 \%$ & $1.00[-5.76,7.76]$ \\
\hline Pohunek 2006b & 201 & $32.9(29.08)$ & 107 & $32(29.08)$ & & $=$ & $41.9 \%$ & $0.90[-5.92,7.72]$ \\
\hline Subtotal $(95 \% \mathrm{CI})$ & 518 & & 315 & & & - & $100.0 \%$ & $1.30[-3.12,5.71]$ \\
\hline \multicolumn{9}{|c|}{ Heterogeneity: Tau ${ }^{2}=0.0: \mathrm{Ch}^{2}=0.13, \mathrm{df}=2(\mathrm{P}=0.94): 1^{2}=0.0 \%$} \\
\hline \multicolumn{9}{|c|}{ Test for overal effect: $Z=0.57(P=0.57)$} \\
\hline \multicolumn{9}{|c|}{2 Mean baseline FEV I $61-79 \%$ of predicted } \\
\hline Subtotal $(95 \% \mathrm{CI})$ & 0 & & $\mathbf{0}$ & & & & & Not estimable \\
\hline \multicolumn{9}{|c|}{ Heterogeneity, not applicable } \\
\hline \multicolumn{9}{|c|}{ Test for overal effect: not applicable } \\
\hline Total $(95 \% \mathrm{CI})$ & 518 & & 315 & & & • & $100.0 \%$ & $1.30[-3.12,5.71]$ \\
\hline \multicolumn{9}{|c|}{ Heterogeneity: $\mathrm{Tau}^{2}=0.0 ; \mathrm{Chi}^{2}=0.13, \mathrm{df}=2(\mathrm{P}=0.94) ; 1^{2}=0.0 \%$} \\
\hline \multicolumn{9}{|c|}{ Test for overal effect: $Z=0.57(P=0.57)$} \\
\hline & & & & & -50 & 50 & $\infty$ & \\
\hline & & & & & Salone & Farours L & $A+1 C S$ & \\
\hline
\end{tabular}




\section{Analysis 1.27}

Comparison 1 Long-acting beta 2 versus placebo: both groups receiving similar dose ICS, Outcome $27 \%$ symptom-free days

Review: Addition of long-acting beta-agonists to inhaled corticosteroids for chronic asthma in children

Comparison: 1 Long-acting beta 2 versus placebo: both groups receiving similar dose ICS Outcome: $27 \%$ symptom-free days

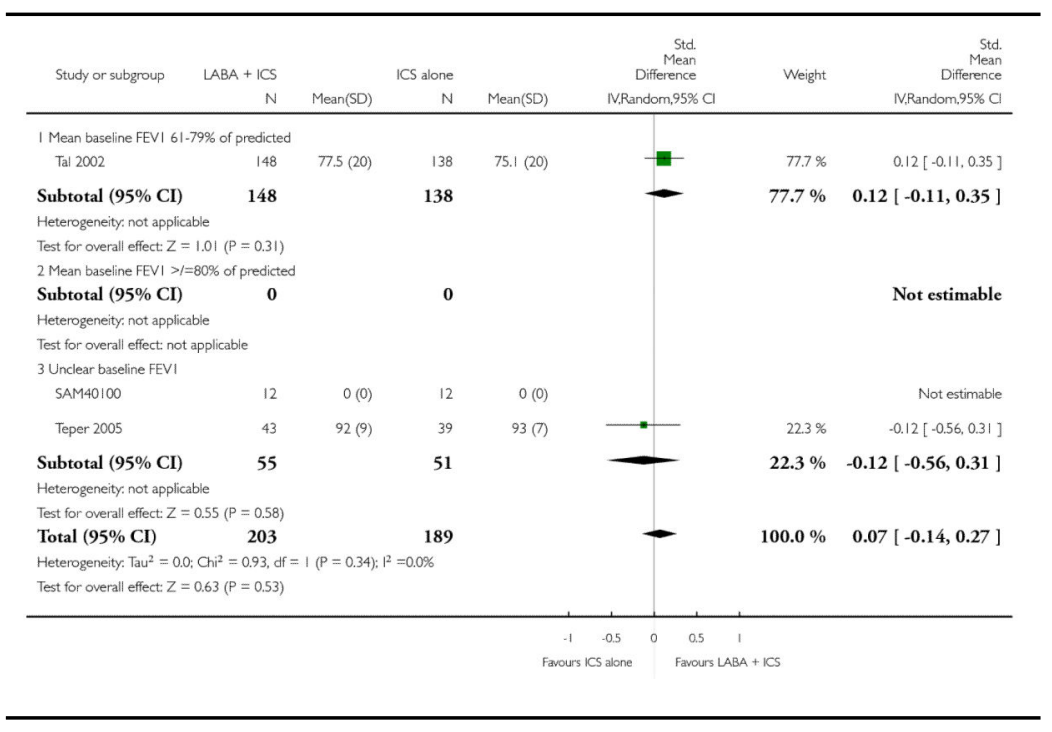

Analysis 1.28

Comparison 1 Long-acting beta 2 versus placebo: both groups receiving similar dose ICS, Outcome $28 \%$ symptom-free nights at $52+/-4$ weeks

Review: Addition of long-acting beta-agonists to inhaled corticosteroids for chronic asthma in children

Comparison: 1 Long-acting beta 2 versus placebo: both groups receiving similar dose ICS Outcome: $28 \%$ symptom-free nights at $52+/-4$ weeks

\begin{tabular}{|c|c|c|c|c|c|c|c|}
\hline Study or subgroup & $\begin{array}{r}\angle A B A+I C S \\
N\end{array}$ & \multicolumn{3}{|c|}{ ICS alone } & \multicolumn{2}{|c|}{$\begin{array}{l}\text { Mean } \\
\text { Diference } \\
\text { IVFixed } 95 \% \text { C }\end{array}$} & $\begin{array}{r}\text { Mean } \\
\text { Difference } \\
\text { IV.Fixed,95\% C }\end{array}$ \\
\hline \multicolumn{8}{|c|}{ | Mean Baseline FEV| not reported } \\
\hline Teper 2005 & 43 & $95(6)$ & 39 & $95(5)$ & & & $0.0[-2.38,2.38]$ \\
\hline & & & & & $\begin{array}{l}-100 \quad-50 \\
\text { Favours ICS alone }\end{array}$ & $\begin{array}{l}50 \\
\text { Farours }\end{array}$ & \\
\hline
\end{tabular}




\section{Analysis 1.29}

Comparison 1 Long-acting beta 2 versus placebo: both groups receiving similar dose ICS, Outcome 29 Quality of life (P-AQLQ)

Review: Addition of long-acting beta-agonists to inhaled corticosteroids for chronic asthma in children

Comparison: 1 Long-acting beta2 versus placebo: both groups receiving similar dose ICS Outcome: 29 Quality of life (P-AQLQ)

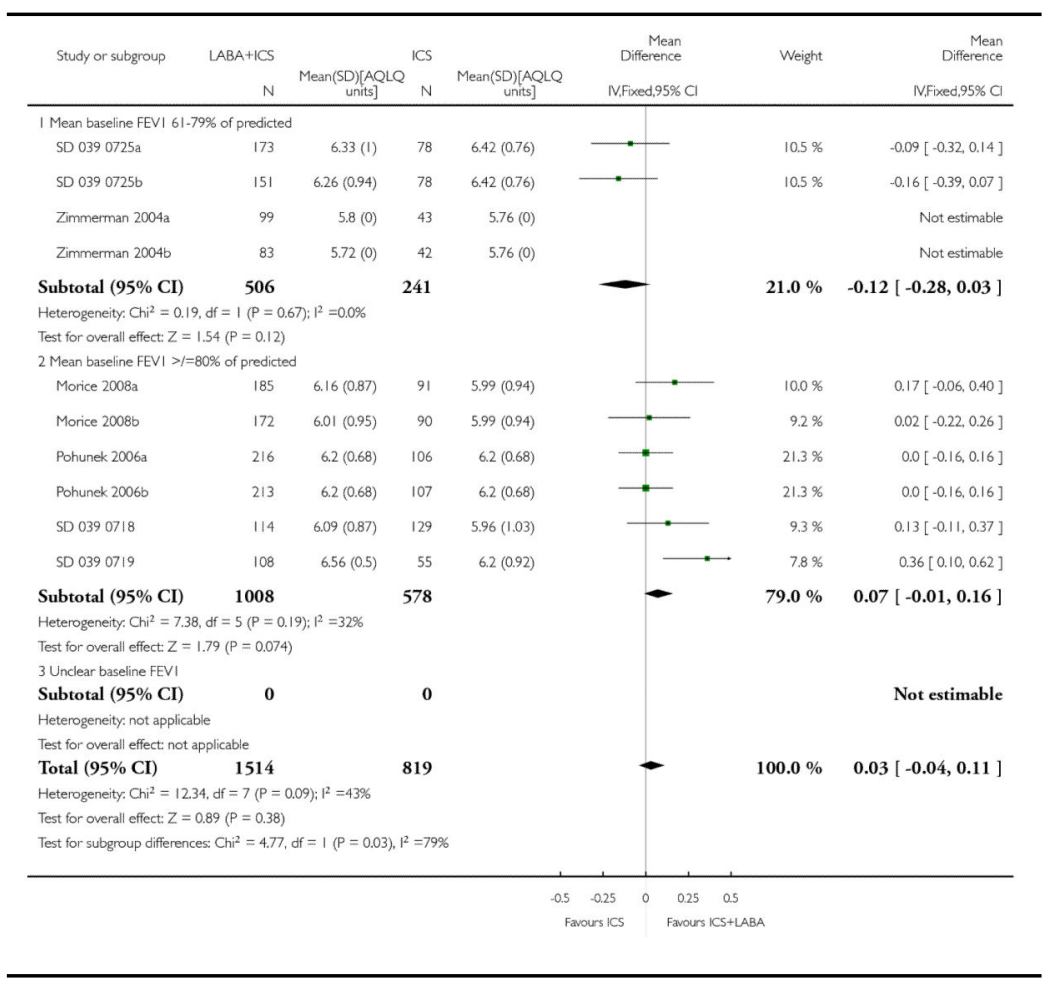




\section{Analysis 1.30}

Comparison 1 Long-acting beta2 versus placebo: both groups receiving similar dose ICS, Outcome 30 Total \# adverse events

Review: Addition of long-acting beta-agonists to inhaled corticosteroids for chronic asthma in children

Comparison: 1 Long-acting beta 2 versus placebo: both groups receiving similar dose ICS Outcome: 30 Total \# adverse events

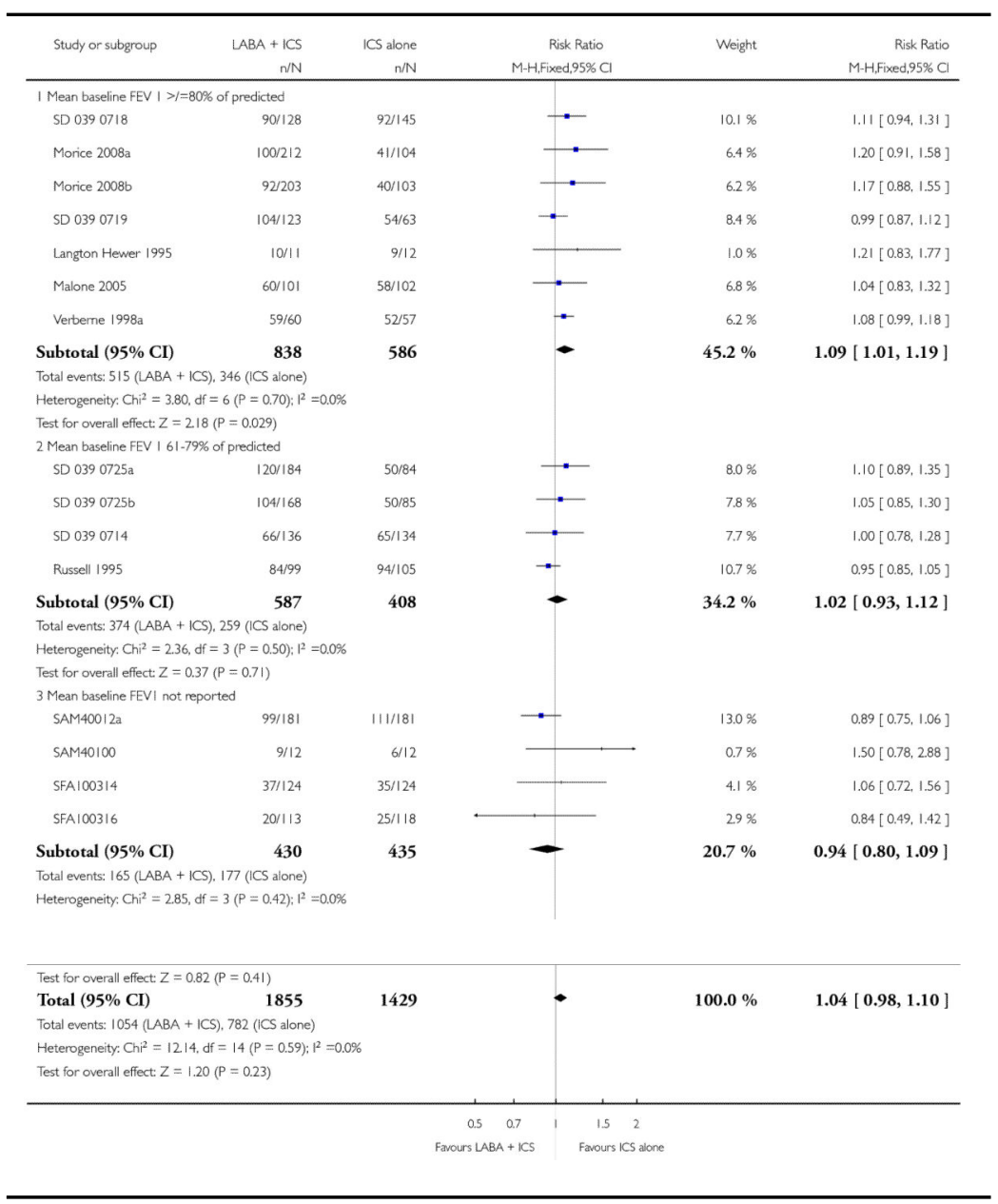




\section{Analysis 1.31}

Comparison 1 Long-acting beta2 versus placebo: both groups receiving similar dose ICS, Outcome 31 \# patients with headache

Review: Addition of long-acting beta-agonists to inhaled corticosteroids for chronic asthma in children

Comparison: 1 Long-acting beta2 versus placebo: both groups receiving similar dose ICS Outcome: 31 \# patients with headache

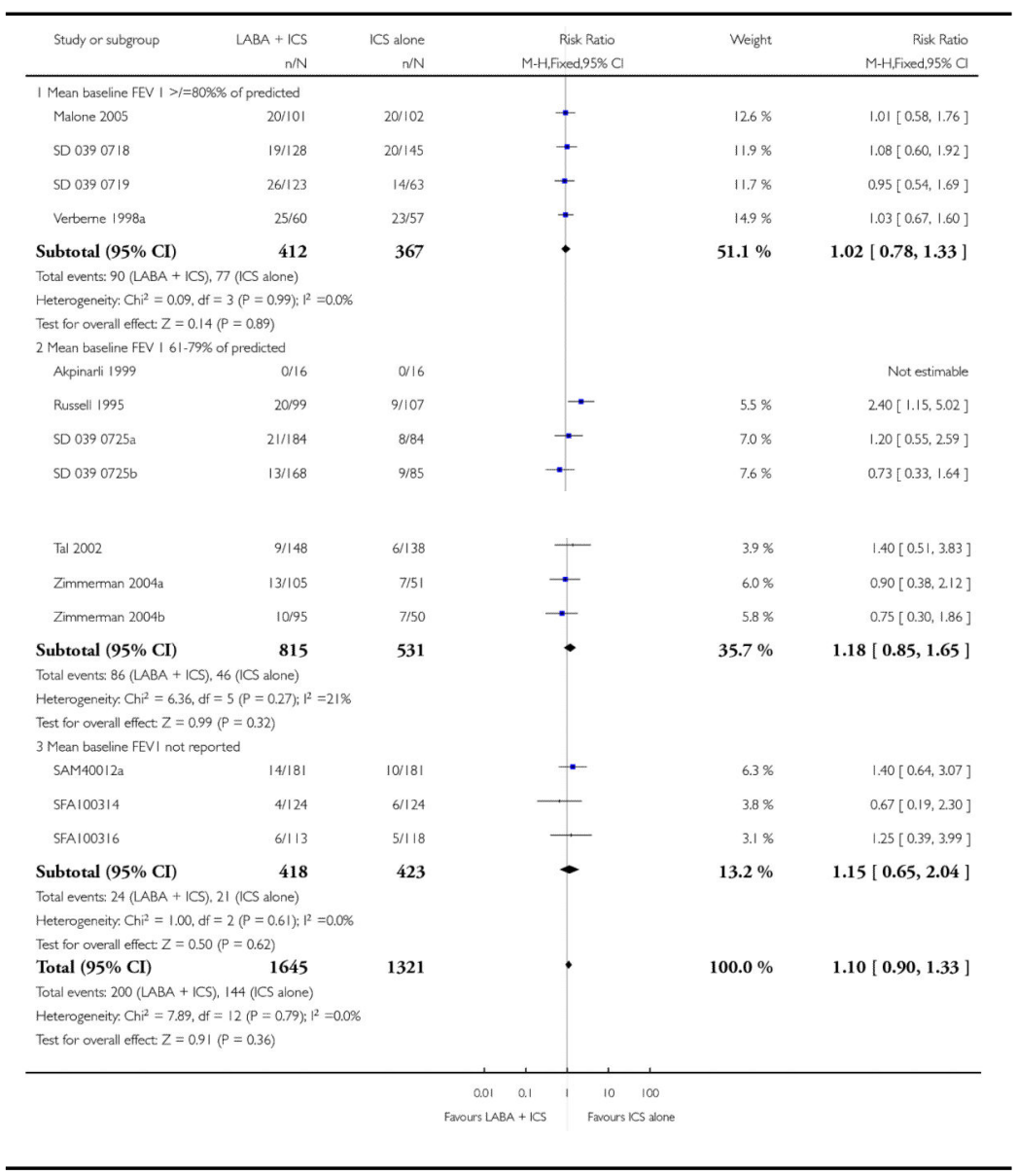




\section{Analysis 1.32}

Comparison 1 Long-acting beta2 versus placebo: both groups receiving similar dose ICS, Outcome 32 \# patients with oral thrush

Review: Addition of long-acting beta-agonists to inhaled corticosteroids for chronic asthma in children

Comparison: 1 Long-acting beta2 versus placebo: both groups receiving similar dose ICS Outcome: 32 \# patients with oral thrush

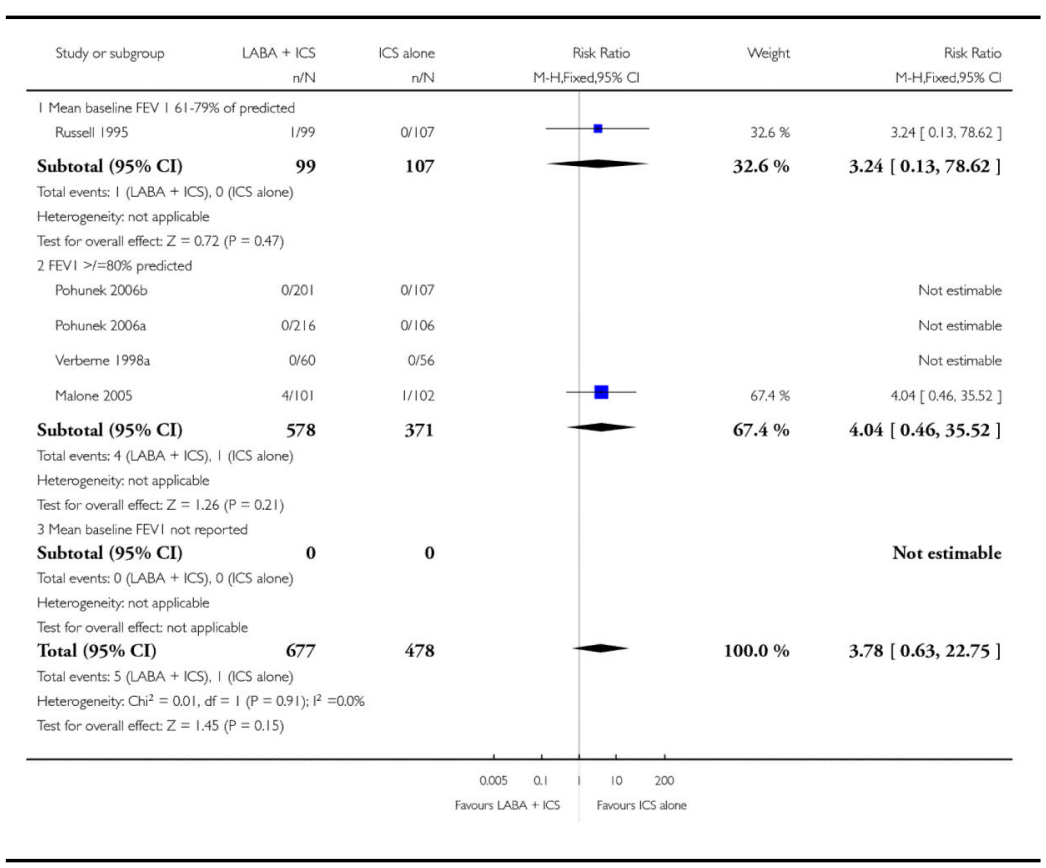




\section{Analysis 1.33}

Comparison 1 Long-acting beta2 versus placebo: both groups receiving similar dose ICS, Outcome 33 \# patients with tremor

Review: Addition of long-acting beta-agonists to inhaled corticosteroids for chronic asthma in children

Comparison: 1 Long-acting beta2 versus placebo: both groups receiving similar dose ICS Outcome: 33 \# patients with tremor

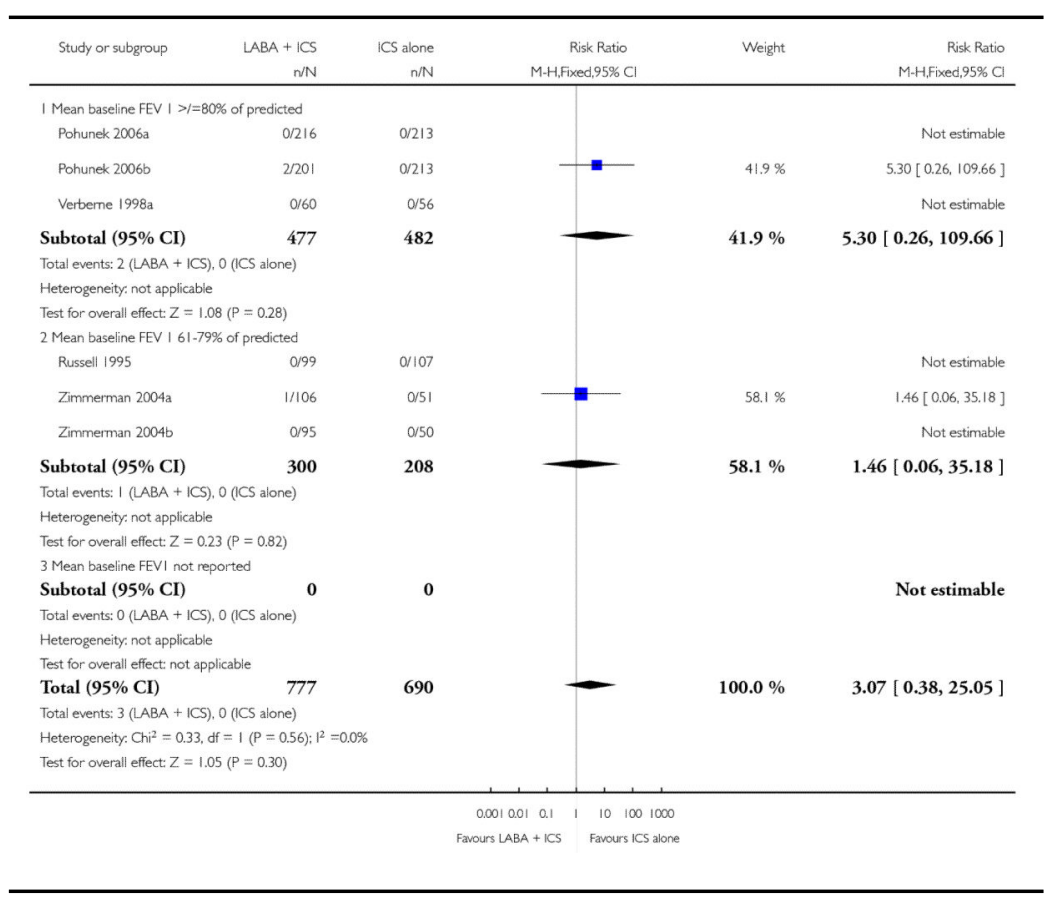




\section{Analysis 1.34}

Comparison 1 Long-acting beta2 versus placebo: both groups receiving similar dose ICS, Outcome 34 \# patients with tachycardia or palpitations

Review: Addition of long-acting beta-agonists to inhaled corticosteroids for chronic asthma in children

Comparison: 1 Long-acting beta2 versus placebo: both groups receiving similar dose ICS Outcome: 34 \# patients with tachycardia or palpitations

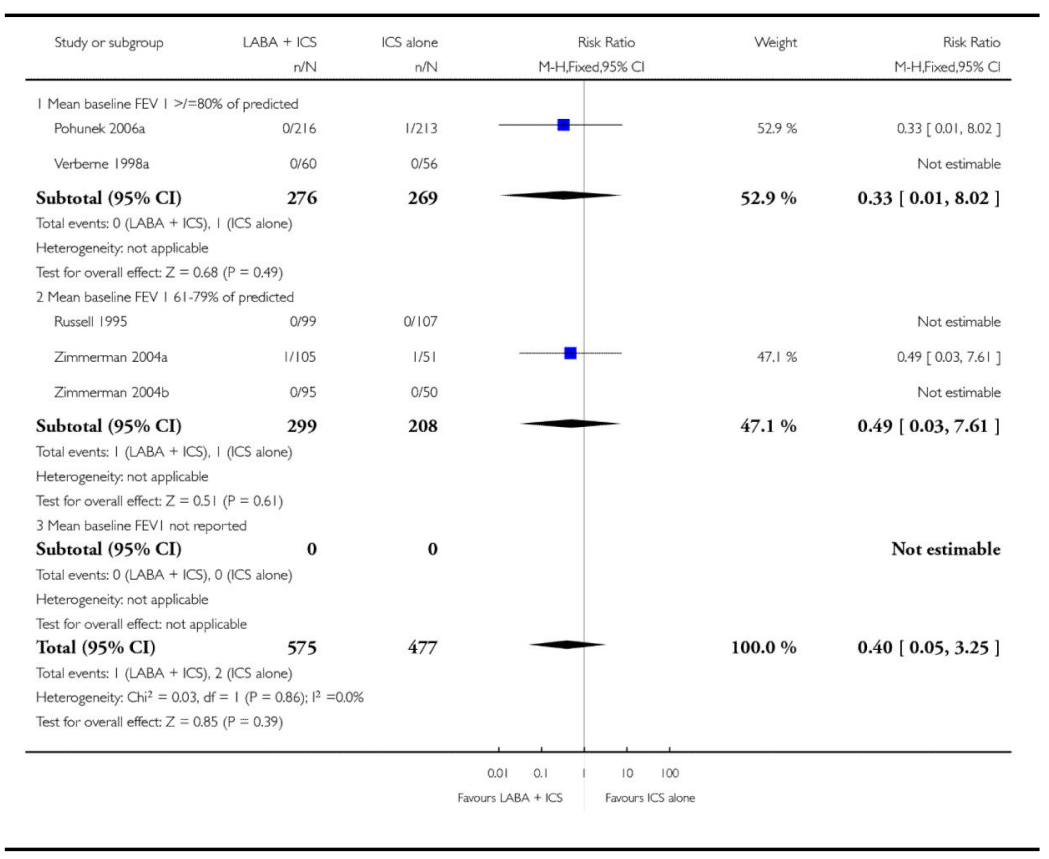




\section{Analysis 1.35}

Comparison 1 Long-acting beta2 versus placebo: both groups receiving similar dose ICS, Outcome 35 \# patients with adverse cardiovascular events

Review: Addition of long-acting beta-agonists to inhaled corticosteroids for chronic asthma in children

Comparison: 1 Long-acting beta2 versus placebo: both groups receiving similar dose ICS Outcome: 35 \# patients with adverse cardiovascular events

\begin{tabular}{|c|c|c|c|c|c|c|}
\hline Study or subgroup & $\begin{array}{r}\angle A B A+I C S \\
n / N \\
\end{array}$ & $\begin{array}{r}\text { ICS alone } \\
\mathrm{n} / \mathrm{N}\end{array}$ & \multicolumn{2}{|c|}{$\begin{array}{c}\text { Risk Ratio } \\
\text { M-H,Fixed,95\% CI }\end{array}$} & Weight & $\begin{array}{r}\text { Risk Ratio } \\
\text { M-H,Fixed,95\% CI }\end{array}$ \\
\hline \multicolumn{7}{|c|}{ | Mean baseline FEV $|>|=80 \%$ of preditted } \\
\hline Verberne 1998a & $0 / 60$ & 1/56 & a & & $100.0 \%$ & $0.31[0.01,7.49]$ \\
\hline Subtotal $(95 \% \mathrm{CI})$ & 60 & 56 & & & $100.0 \%$ & $0.31[0.01,7.49]$ \\
\hline \multicolumn{7}{|c|}{ Total events: 0 (LABA + ICS), I (ICS alone) } \\
\hline \multicolumn{7}{|c|}{ Heterogeneity, not applicable } \\
\hline \multicolumn{7}{|c|}{ Test for overall effect: $Z=0.72(P=0.47)$} \\
\hline \multicolumn{7}{|c|}{2 Mean baseline FEV I $61-79 \%$ of predicted } \\
\hline Akpinarli 1999 & $0 / 16$ & $0 / 16$ & & & & Not estimable \\
\hline Subtotal $(95 \% \mathrm{CI})$ & 16 & 16 & & & & Not estimable \\
\hline \multicolumn{7}{|c|}{ Total events: 0 (LABA + ICS), 0 (ICS alone) } \\
\hline \multicolumn{7}{|c|}{ Heterogeneity, not applicable } \\
\hline \multicolumn{7}{|c|}{ Test for overall effect: not applicable } \\
\hline Total $(95 \% \mathrm{CI})$ & 76 & 72 & & & $100.0 \%$ & $0.31[0.01,7.49]$ \\
\hline \multicolumn{7}{|c|}{ Total events: 0 (LABA + ICS), 1 (ICS alone) } \\
\hline \multicolumn{7}{|c|}{ Heterogeneity, not applicable } \\
\hline \multicolumn{7}{|c|}{ Test for overal effect: $Z=0.72(P=0.47)$} \\
\hline & & & $0.01 \quad 0.1$ & $10 \quad 100$ & & \\
\hline & & & Farours $1 A B A+1 C S$ & Favours ICS aione & & \\
\hline
\end{tabular}

Analysis 1.36

Comparison 1 Long-acting beta2 versus placebo: both groups receiving similar dose ICS, Outcome 36 Change in \% PC 20 at endpoint

Review: Addition of long-acting beta-agonists to inhaled corticosteroids for chronic asthma in children

Comparison: 1 Long-acting beta2 versus placebo: both groups receiving similar dose ICS Outcome: 36 Change in \% PC 20 at endpoint

\begin{tabular}{|c|c|c|c|c|c|c|c|}
\hline \multirow[t]{2}{*}{ Study or subgroup } & \multirow{2}{*}{$\begin{array}{r}\angle A B A+I C S \\
N\end{array}$} & \multicolumn{3}{|c|}{ ICS alone } & \multirow{2}{*}{\multicolumn{2}{|c|}{$\begin{array}{c}\text { Mean } \\
\text { Difference } \\
\mathrm{IV}, \mathrm{Fixed}, 95 \% \mathrm{Cl}\end{array}$}} & \multirow{2}{*}{$\begin{array}{r}\text { Mean } \\
\text { Difference } \\
\text { IV,Fixed,95\% C }\end{array}$} \\
\hline & & $\operatorname{Mean}(S D)$ & $\mathrm{N}$ & $\operatorname{Mean}(S D)$ & & & \\
\hline \multicolumn{8}{|c|}{ | Mean baseline |FV $|>|=80 \%$ of predicted } \\
\hline Mejer 1995 & 20 & $1.1(1.34)$ & 19 & $0.8(1.74)$ & & - & $0.30[-0.68,1.28]$ \\
\hline & & & & & $\begin{array}{cc}-10 & -5 \\
\text { Farours ICS alone }\end{array}$ & $\begin{array}{c}5 \\
\text { Farours }\end{array}$ & \\
\hline
\end{tabular}




\section{Analysis 1.37}

Comparison 1 Long-acting beta2 versus placebo: both groups receiving similar dose ICS, Outcome 37 Change in height $(\mathrm{cm})$ as SD scores at $24+/-4$ weeks

Review: Addition of long-acting beta-agonists to inhaled corticosteroids for chronic asthma in children

Comparison: 1 Long-acting beta2 versus placebo: both groups receiving similar dose ICS Outcome: 37 Change in height $(\mathrm{cm})$ as SD scores at $24+/-4$ weeks

\begin{tabular}{|c|c|c|c|c|c|c|c|}
\hline Study or subgroup & $\begin{array}{r}\angle A B A+I C S \\
N\end{array}$ & \multicolumn{3}{|c|}{ ICS alone } & \multicolumn{2}{|c|}{$\begin{array}{c}\text { Mean } \\
\text { Difference } \\
\text { IN.Fixed,95\% CI }\end{array}$} & $\begin{array}{r}\text { Mean } \\
\text { Difference } \\
\text { IVFixed,95\% C }\end{array}$ \\
\hline \multirow{3}{*}{$\begin{array}{l}\text { I Mean baseline FEV } \\
\text { Verberne } 1998 \mathrm{a}\end{array}$} & $=80 \%$ of predic & & & & & & \\
\hline & 60 & $-0.1(0.31)$ & 57 & $-0.16(0.3)$ & & & $0.06[-0.05,0.17]$ \\
\hline & & & & & $\begin{array}{cc}-10 & -5 \\
\text { Favours ICS alone }\end{array}$ & $\begin{array}{c}5 \\
\text { Farours }\end{array}$ & \\
\hline
\end{tabular}

\section{Analysis 1.38}

Comparison 1 Long-acting beta2 versus placebo: both groups receiving similar dose ICS, Outcome 38 Change in height at one year

Review: Addition of long-acting beta-agonists to inhaled corticosteroids for chronic asthma in children

Comparison: 1 Long-acting beta2 versus placebo: both groups receiving similar dose ICS Outcome: 38 Change in height at one year

\begin{tabular}{|c|c|c|c|c|c|c|c|}
\hline Study or subgroup & $\begin{array}{r}\angle A B A+I C S \\
N\end{array}$ & \multicolumn{3}{|c|}{ ICS } & \multicolumn{2}{|c|}{$\begin{array}{r}\text { Mean } \\
\text { Difference }\end{array}$} & $\begin{array}{r}\text { Mean } \\
\text { Difference } \\
\text { N.Fixed,95\% CI }\end{array}$ \\
\hline \multirow[t]{2}{*}{ Verberne 1998a } & 60 & $5.1(2.6)$ & 57 & $4.5(2.6)$ & & $\rightarrow$ & $0.60[-0.34,1.54]$ \\
\hline & & & & & $\begin{array}{cc}.10 & -5 \\
\text { Favours treatment }\end{array}$ & $\begin{array}{cc}0 & 5 \\
& \text { Farours }\end{array}$ & \\
\hline
\end{tabular}




\section{Analysis 2.1}

Comparison 2 Long-acting beta 2 agonist + ICS versus placebo + higher dose of ICS, Outcome 1 \# patients with exacerbations requiring oral steroids

Review: Addition of long-acting beta-agonists to inhaled corticosteroids for chronic asthma in children

Comparison: 2 Long-acting beta2 agonist + ICS versus placebo + higher dose of ICS Outcome: 1 \# patients with exacerbations requiring oral steroids

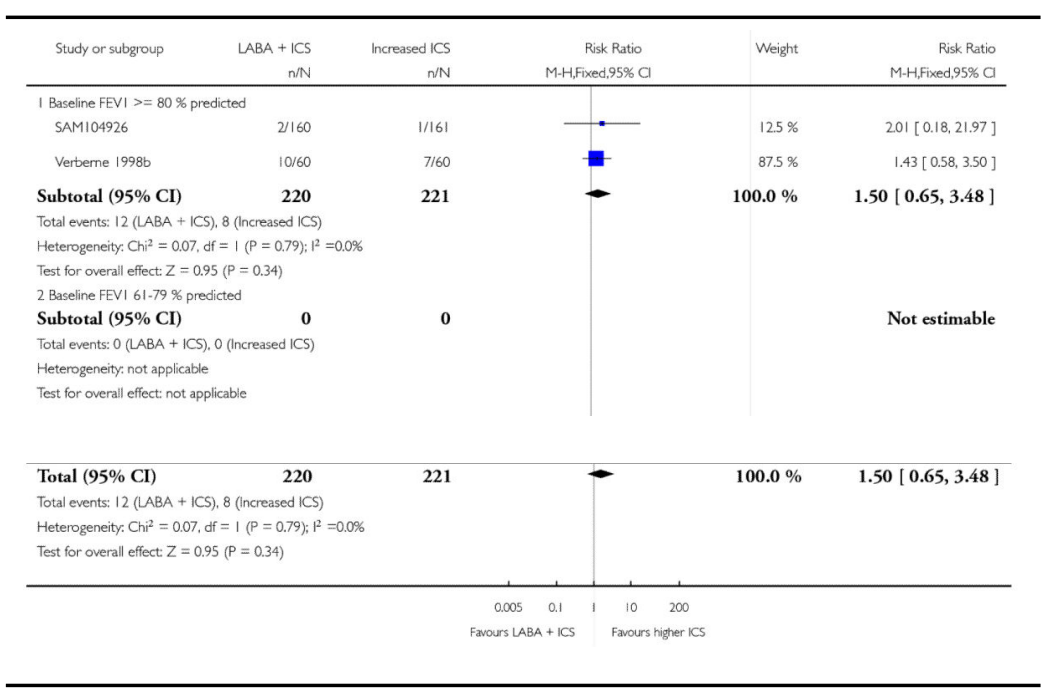




\section{Analysis 2.2}

Comparison 2 Long-acting beta 2 agonist + ICS versus placebo + higher dose of ICS, Outcome 2 \# patients with exacerbations requiring hospitalisation

Review: Addition of long-acting beta-agonists to inhaled corticosteroids for chronic asthma in children

Comparison: 2 Long-acting beta2 agonist + ICS versus placebo + higher dose of ICS

Outcome: 2 \# patients with exacerbations requiring hospitalisation

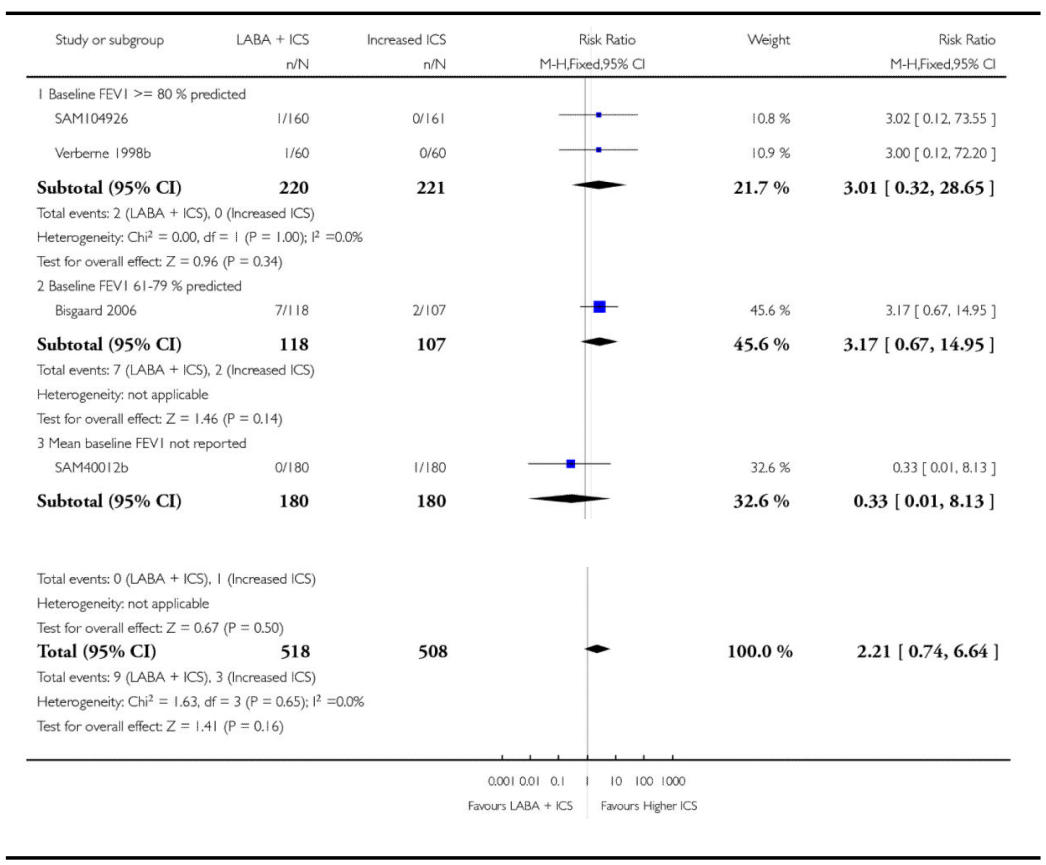




\section{Analysis 2.3}

Comparison 2 Long-acting beta 2 agonist + ICS versus placebo + higher dose of ICS, Outcome 3 Serious adverse events

Review: Addition of long-acting beta-agonists to inhaled corticosteroids for chronic asthma in children

Comparison: 2 Long-acting beta2 agonist + ICS versus placebo + higher dose of ICS Outcome: 3 Serious adverse events

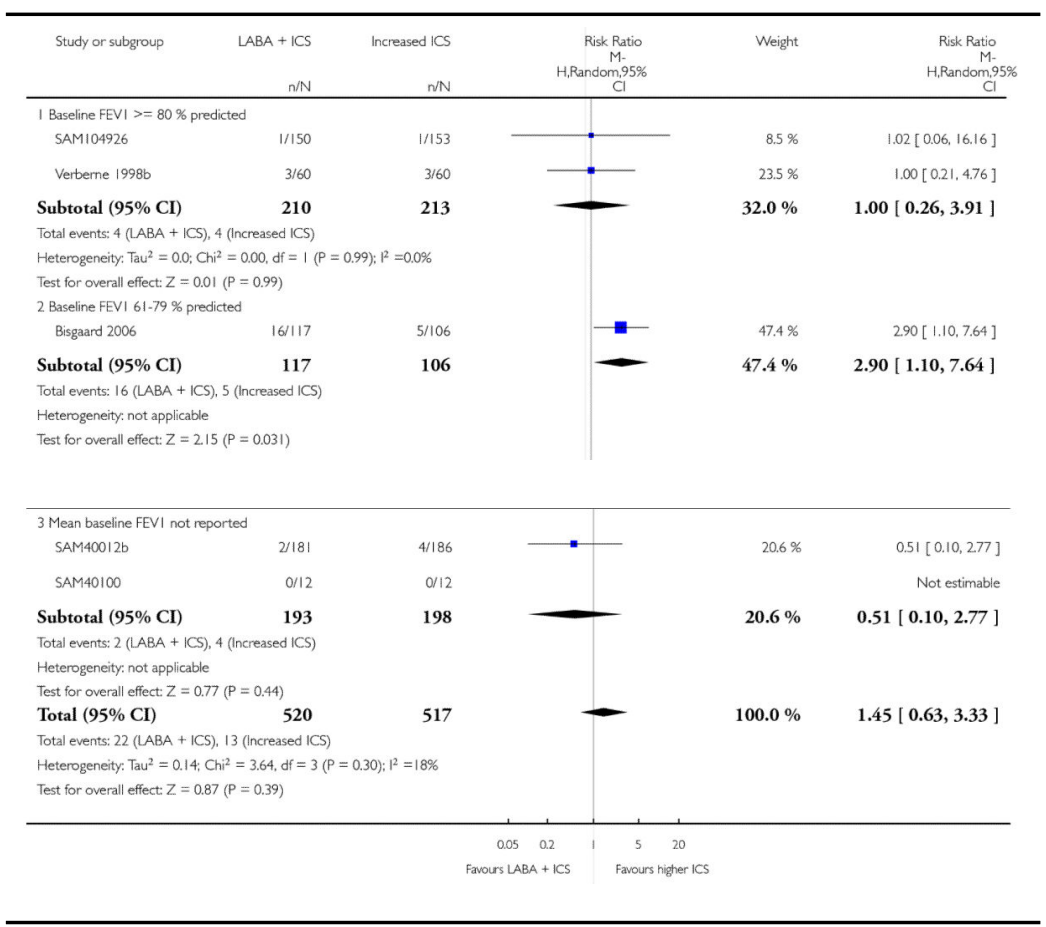


Analysis 2.4

Comparison 2 Long-acting beta 2 agonist + ICS versus placebo + higher dose of ICS, Outcome 4 Total \# withdrawals

Review: Addition of long-acting beta-agonists to inhaled corticosteroids for chronic asthma in children

Comparison: 2 Long-acting beta 2 agonist + ICS versus placebo + higher dose of ICS Outcome: 4 Total \# withdrawals

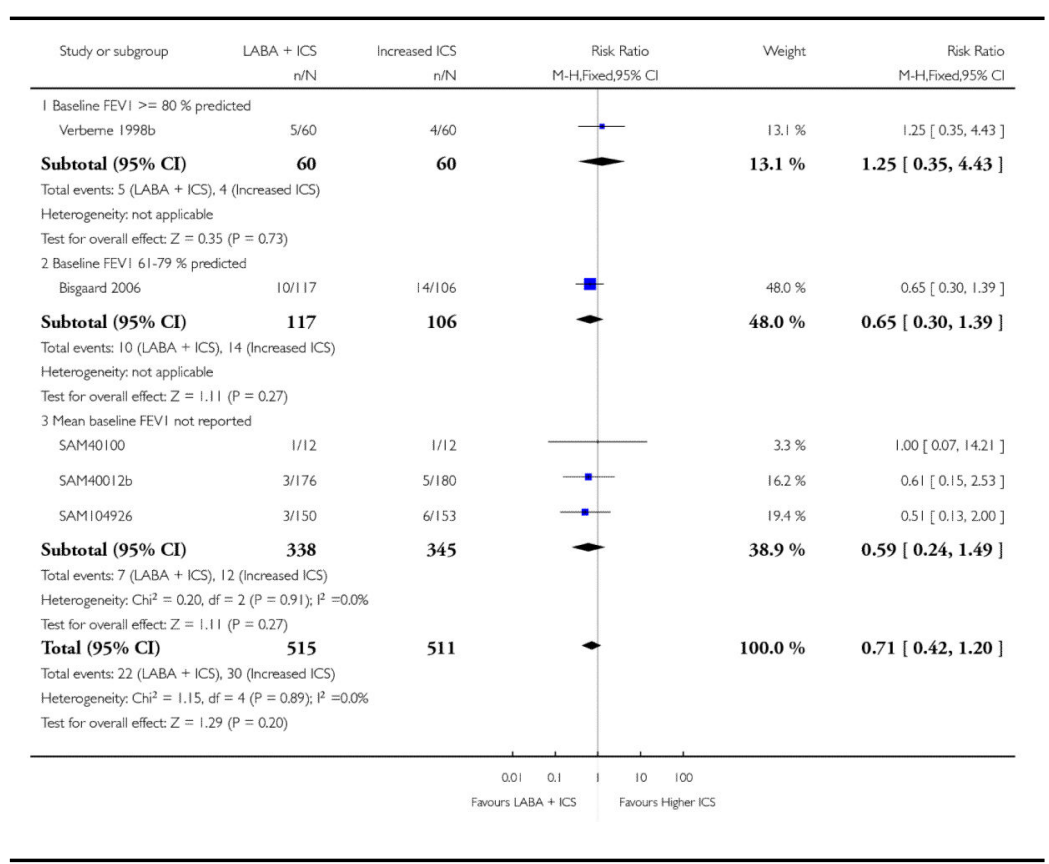




\section{Analysis 2.5}

Comparison 2 Long-acting beta 2 agonist + ICS versus placebo + higher dose of ICS, Outcome 5 \# withdrawals due to adverse events

Review: Addition of long-acting beta-agonists to inhaled corticosteroids for chronic asthma in children

Comparison: 2 Long-acting beta2 agonist + ICS versus placebo + higher dose of ICS

Outcome: 5 \# withdrawals due to adverse events

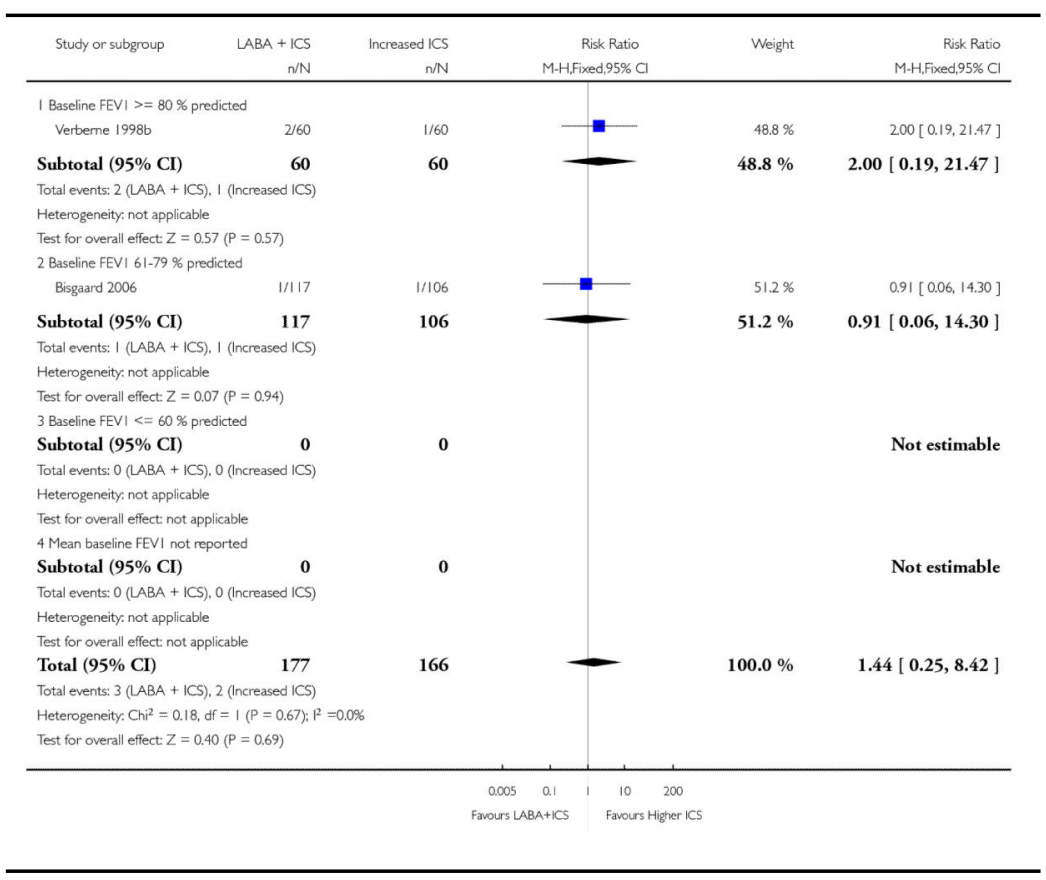

Analysis 2.6

Comparison 2 Long-acting beta 2 agonist + ICS versus placebo + higher dose of ICS, Outcome 6 \# withdrawals due to poor asthma control or exacerbation

Review: Addition of long-acting beta-agonists to inhaled corticosteroids for chronic asthma in children

Comparison: 2 Long-acting beta2 agonist + ICS versus placebo + higher dose of ICS Outcome: 6 \# withdrawals due to poor asthma control or exacerbation

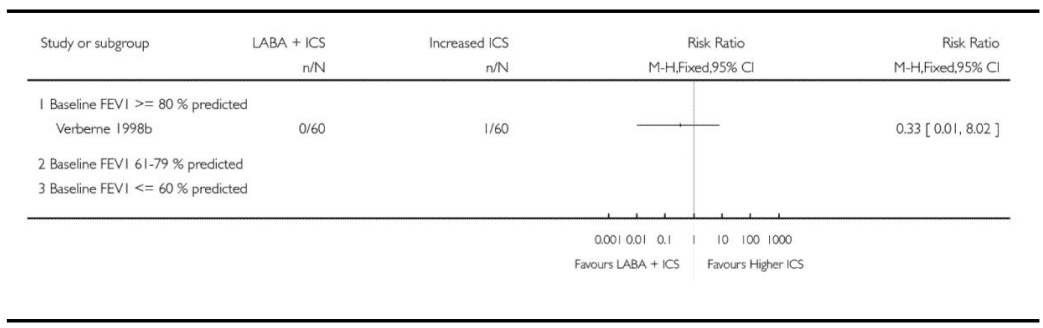




\section{Analysis 2.7}

Comparison 2 Long-acting beta 2 agonist + ICS versus placebo + higher dose of ICS, Outcome 7 Change in FEV1 at endpoint

Review: Addition of long-acting beta-agonists to inhaled corticosteroids for chronic asthma in children

Comparison: 2 Long-acting beta2 agonist + ICS versus placebo + higher dose of ICS Outcome: 7 Change in FEV1 at endpoint

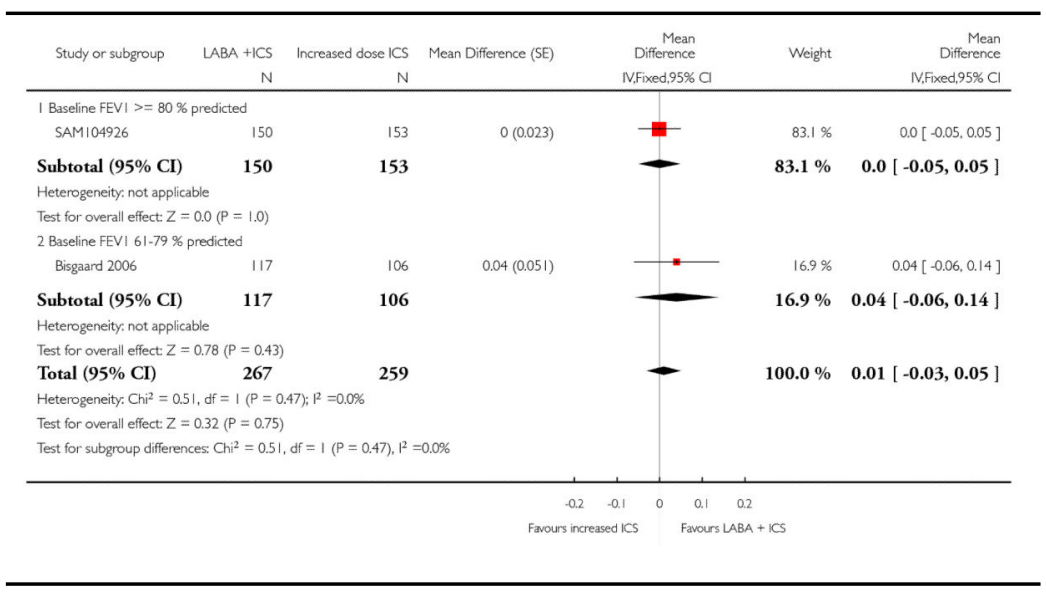

Analysis 2.8

Comparison 2 Long-acting beta 2 agonist + ICS versus placebo + higher dose of ICS, Outcome 8 FEV1 at endpoint

Review: Addition of long-acting beta-agonists to inhaled corticosteroids for chronic asthma in children

Comparison: 2 Long-acting beta2 agonist + ICS versus placebo + higher dose of ICS Outcome: 8 FEV1 at endpoint

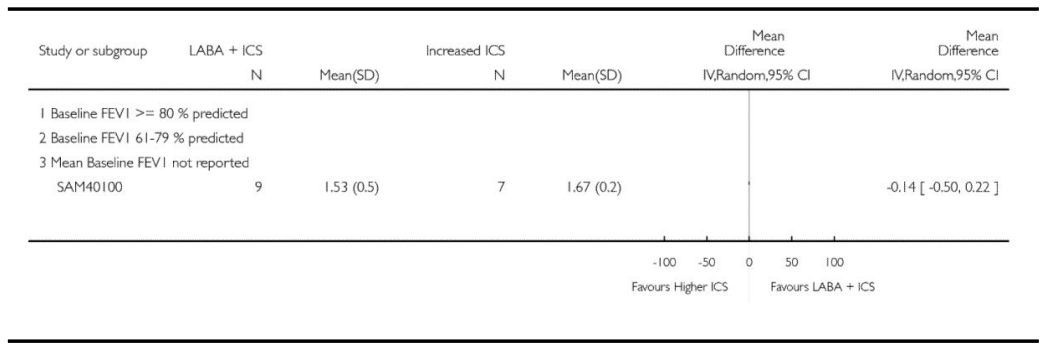




\section{Analysis 2.9}

Comparison 2 Long-acting beta 2 agonist + ICS versus placebo + higher dose of ICS, Outcome 9 Change in FEV1 predicted at endpoint

Review: Addition of long-acting beta-agonists to inhaled corticosteroids for chronic asthma in children

Comparison: 2 Long-acting beta2 agonist + ICS versus placebo + higher dose of ICS Outcome: 9 Change in FEV1 predicted at endpoint

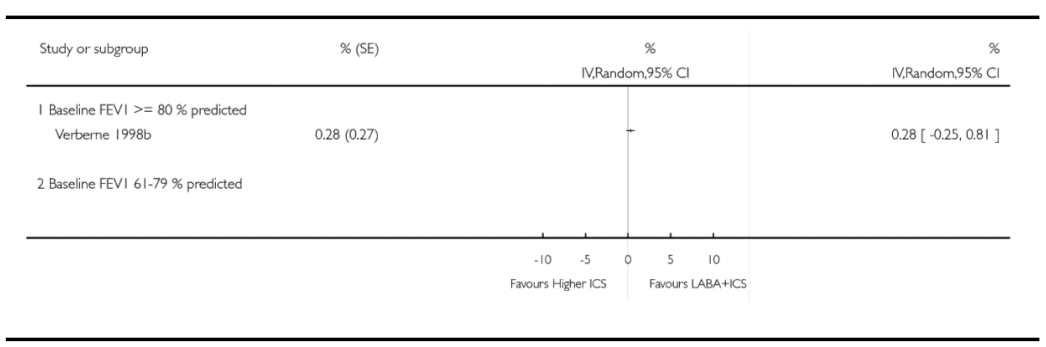

\section{Analysis 2.10}

Comparison 2 Long-acting beta 2 agonist + ICS versus placebo + higher dose of ICS, Outcome 10 Change in morning PEF (L/min) at endpoint

Review: Addition of long-acting beta-agonists to inhaled corticosteroids for chronic asthma in children

Comparison: 2 Long-acting beta2 agonist + ICS versus placebo + higher dose of ICS Outcome: 10 Change in morning PEF (L/min) at endpoint

\begin{tabular}{|c|c|c|c|c|c|c|c|c|}
\hline \multirow[t]{2}{*}{ Study or subgroup } & \multirow{2}{*}{$\begin{array}{r}\angle A B A+I C S \\
N\end{array}$} & \multicolumn{3}{|c|}{ Increased ICS } & \multirow{2}{*}{\multicolumn{2}{|c|}{$\begin{array}{c}\text { Mean } \\
\text { Difference } \\
\text { IV.Random, } 95 \% \mathrm{CI}\end{array}$}} & \multirow[t]{2}{*}{ Weight } & \multirow{2}{*}{$\begin{array}{r}\text { Mean } \\
\text { Difference } \\
\text { N.Random,95\% Cl }\end{array}$} \\
\hline & & $\operatorname{Mean}(\mathrm{SD})$ & $\mathrm{N}$ & $\operatorname{Mean}(\mathrm{SD})$ & & & & \\
\hline \multicolumn{9}{|c|}{ | Baseline FEV| >= $80 \%$ predicted } \\
\hline Verberne $1998 \mathrm{~b}$ & 60 & $30.92(33)$ & 60 & $22.57(33)$ & & $\rightarrow$ & $11.4 \%$ & $8.35[-3.46,20.16]$ \\
\hline SAM104926 & 150 & $26.9(26.09)$ & 153 & $19.3(26.22)$ & & $\mathbf{m}$ & $45.7 \%$ & $7.60[1.71,13.49]$ \\
\hline Subtotal $(95 \% \mathrm{CI})$ & 210 & & 213 & & & - & $57.0 \%$ & $7.75[2.48,13.02]$ \\
\hline \multicolumn{9}{|c|}{ Heterogeneity. $\mathrm{Tau}^{2}=0.0 ; \mathrm{Chi}^{2}=0.01, \mathrm{df}=1 \quad(\mathrm{P}=0.91) ; \mathrm{R}^{2}=0.0 \%$} \\
\hline \multicolumn{9}{|c|}{ Test for overal effect: $Z=2.88(P=0.0040)$} \\
\hline \multicolumn{9}{|c|}{2 Baseline FEVI $61.79 \%$ predicted } \\
\hline Bisgaard 2006 & 117 & $26(34.51)$ & 106 & $17(34.51)$ & & * & $19.3 \%$ & $9.00[-0.07,18.07]$ \\
\hline Subtotal $(95 \% \mathrm{CI})$ & 117 & & 106 & & & - & $19.3 \%$ & $9.00[-0.07,18.07]$ \\
\hline \multicolumn{9}{|c|}{ Heterogeneity, not applicable } \\
\hline \multicolumn{9}{|c|}{ Test for overall effect: $Z=1.94(P=0.052)$} \\
\hline \multicolumn{9}{|c|}{3 Mean Baseline FEVI not reported } \\
\hline SAM40012b & 176 & $45.1(39.8)$ & 180 & $39.2(38.9)$ & & * & $23.7 \%$ & $5.90[-2.28,14.08]$ \\
\hline Subtotal $(95 \% \mathrm{CI})$ & 176 & & 180 & & & - & $23.7 \%$ & $5.90[-2.28,14.08]$ \\
\hline \multicolumn{9}{|c|}{ Heterogeneity, not applicable } \\
\hline \multicolumn{9}{|c|}{ Test for overall effect: $Z=1.41(P=0.16)$} \\
\hline Total $(95 \% \mathrm{CI})$ & 503 & & 499 & & & - & $100.0 \%$ & $7.55[3.57,11.53]$ \\
\hline \multirow{2}{*}{\multicolumn{9}{|c|}{$\begin{array}{l}\text { Heterogeneity, } \mathrm{Tau}^{2}=0.0 ; \mathrm{Ch}^{2}=0.27, \mathrm{df}=3(\mathrm{P}=0.97), \mathrm{P}^{2}=0.0 \% \\
\text { Test for overal effect: } Z=3.72(\mathrm{P}=0.00020)\end{array}$}} \\
\hline \multicolumn{6}{|c|}{ Test for overal effect: $Z=3.72(P=0.00020)$} & & & \\
\hline & & & & & $\begin{array}{l}.50 \\
-50 \\
\text { erics }\end{array}$ & $\begin{array}{c}0 \\
0 \\
\text { Favours L L }\end{array}$ & $A+1 C S$ & \\
\hline
\end{tabular}




\section{Analysis 2.11}

\section{Comparison 2 Long-acting beta 2 agonist + ICS versus placebo + higher dose of ICS, Outcome 11 Change in pm PEF}

Review: Addition of long-acting beta-agonists to inhaled corticosteroids for chronic asthma in children

Comparison: 2 Long-acting beta2 agonist + ICS versus placebo + higher dose of ICS Outcome: 11 Change in pm PEF

\begin{tabular}{|c|c|c|c|c|c|c|c|}
\hline Study or subgroup & $\begin{array}{r}\text { LABA and ICS } \\
\mathrm{N}\end{array}$ & $\begin{array}{r}\text { Increased dose ICS } \\
\mathrm{N} \\
\end{array}$ & Mean Difference (SE) & & $\begin{array}{l}\text { Mean } \\
\text { Eerence } \\
\text { ed,95\% Cl } \\
\end{array}$ & Weight & $\begin{array}{r}\text { Mean } \\
\text { Difference } \\
\text { IV,Fixed,95\% Cl }\end{array}$ \\
\hline \multicolumn{8}{|c|}{ | Baseline FEVI predicted >=80\% } \\
\hline SAM104926 & 150 & 153 & $5.4(3.04)$ & & \pm & $51.8 \%$ & $5.40[-0.56,11.36]$ \\
\hline Subtotal $(95 \% \mathrm{CI})$ & 150 & 153 & & & - & $51.8 \%$ & $5.40[-0.56,11.36]$ \\
\hline \multicolumn{8}{|c|}{ Heterogeneity. not applicable } \\
\hline \multicolumn{8}{|c|}{ Test for overal effect: $Z=1.78(P=0.076)$} \\
\hline \multicolumn{8}{|c|}{2 Baseline FEVI $61.79 \%$ predicted } \\
\hline Bisgaard 2006 & 117 & 106 & $7(4.6276)$ & & * & $22.4 \%$ & $7.00[-2.07,16.07]$ \\
\hline Subtotal $(95 \% \mathrm{CI})$ & 117 & 106 & & & - & $22.4 \%$ & $7.00[-2.07,16.07]$ \\
\hline \multicolumn{8}{|c|}{ Heterogeneity not applicable } \\
\hline \multicolumn{8}{|c|}{ Test for overall effect: $Z=1.51(P=0.13)$} \\
\hline \multicolumn{8}{|c|}{3 Mean Baseline FEVI not reported } \\
\hline SAM40012b & 176 & 180 & $4.4(4.3112)$ & & 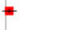 & $25.8 \%$ & $4.40[-4.05,12.85]$ \\
\hline Subtotal $(95 \% \mathrm{CI})$ & 176 & 180 & & & - & $25.8 \%$ & $4.40[-4.05,12.85]$ \\
\hline \multicolumn{8}{|c|}{ Heterogeneity. not applicable } \\
\hline \multicolumn{8}{|c|}{ Test for overall effect: $Z=1.02(P=0.31)$} \\
\hline Total $(95 \% \mathrm{CI})$ & 443 & 439 & & & - & $100.0 \%$ & $5.50[1.21,9.79]$ \\
\hline \multicolumn{8}{|c|}{ Heterogeneity: $C \mathrm{~h}^{2}=0.17, \mathrm{df}=2(\mathrm{P}=0.92) ; \mathrm{I}^{2}=0.0 \%$} \\
\hline \multicolumn{8}{|c|}{ Test for overall effect: $Z=2.51(P=0.012)$} \\
\hline \multicolumn{8}{|c|}{ Test for subgroup differences: $C h i^{2}=0.17, \mathrm{df}=2(P=0.92), l^{2}=0.0 \%$} \\
\hline & & & Favours & $\begin{array}{l}.50 \\
\text { ed ICS }\end{array}$ & $\begin{array}{l}0 \\
0\end{array}$ & $4+1 C S$ & \\
\hline
\end{tabular}

Analysis 2.12

Comparison 2 Long-acting beta 2 agonist + ICS versus placebo + higher dose of ICS, Outcome 12 Change in clinic PEF (L/min)

Review: Addition of long-acting beta-agonists to inhaled corticosteroids for chronic asthma in children

Comparison: 2 Long-acting beta2 agonist + ICS versus placebo + higher dose of ICS Outcome: 12 Change in clinic PEF (L/min)

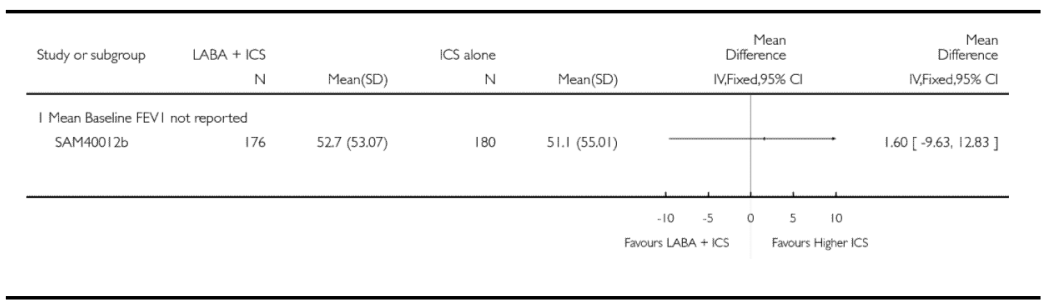




\section{Analysis 2.13}

Comparison 2 Long-acting beta2 agonist + ICS versus placebo + higher dose of ICS, Outcome 13 \# daytime rescue inhalations at endpoint

Review: Addition of long-acting beta-agonists to inhaled corticosteroids for chronic asthma in children

Comparison: 2 Long-acting beta2 agonist + ICS versus placebo + higher dose of ICS

Outcome: 13 \# daytime rescue inhalations at endpoint

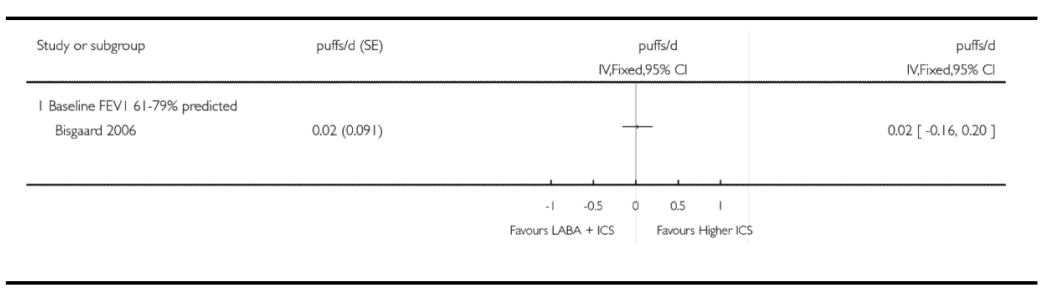

Analysis 2.14

Comparison 2 Long-acting beta2 agonist + ICS versus placebo + higher dose of ICS, Outcome 14 Number of nighttime awakenings

Review: Addition of long-acting beta-agonists to inhaled corticosteroids for chronic asthma in children

Comparison: 2 Long-acting beta2 agonist + ICS versus placebo + higher dose of ICS Outcome: 14 Number of nighttime awakenings

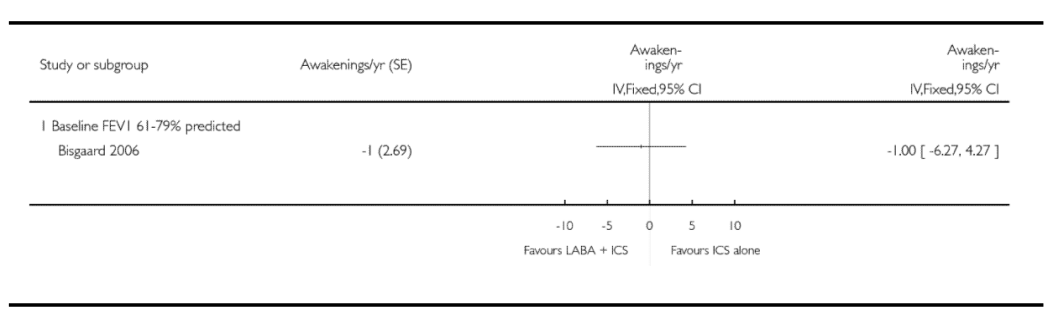




\section{Analysis 2.15}

Comparison 2 Long-acting beta2 agonist + ICS versus placebo + higher dose of ICS, Outcome 15 Total \# adverse events

Review: Addition of long-acting beta-agonists to inhaled corticosteroids for chronic asthma in children

Comparison: 2 Long-acting beta2 agonist + ICS versus placebo + higher dose of ICS Outcome: 15 Total \# adverse events

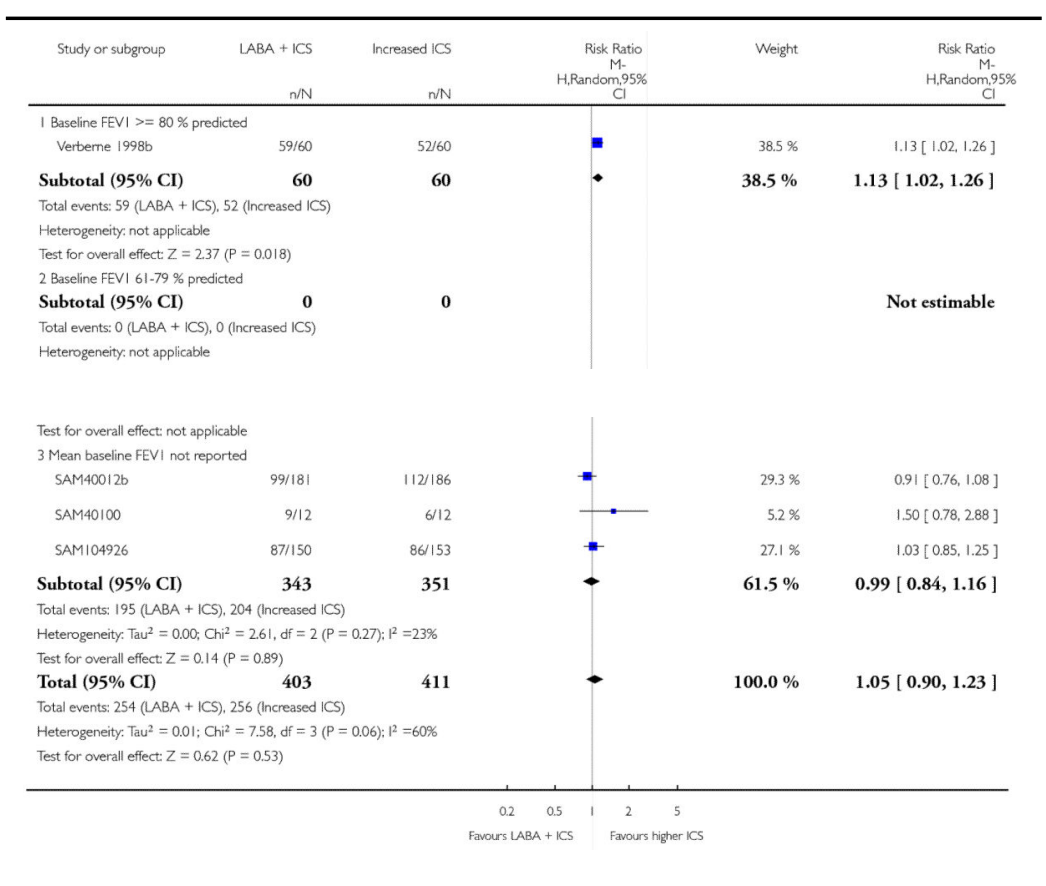




\section{Analysis 2.16}

Comparison 2 Long-acting beta 2 agonist + ICS versus placebo + higher dose of ICS, Outcome 16 \# patients with headache

Review: Addition of long-acting beta-agonists to inhaled corticosteroids for chronic asthma in children

Comparison: 2 Long-acting beta2 agonist + ICS versus placebo + higher dose of ICS Outcome: 16 \# patients with headache

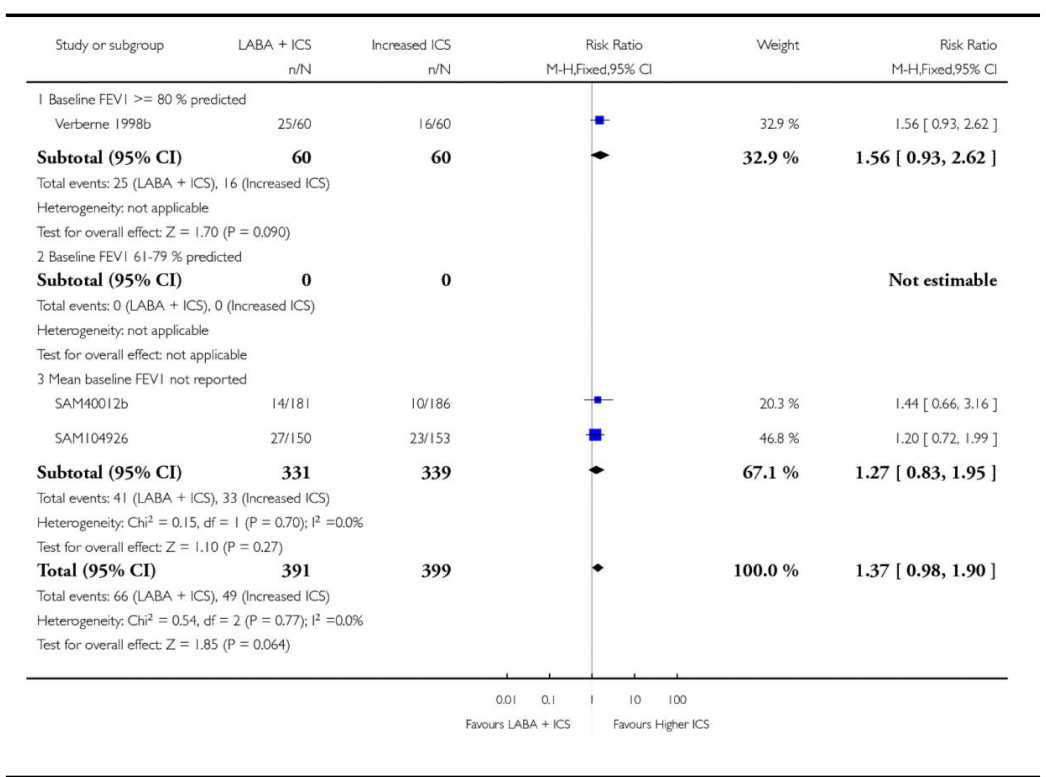

\section{Analysis 2.17}

Comparison 2 Long-acting beta 2 agonist + ICS versus placebo + higher dose of ICS, Outcome 17 Linear growth

Review: Addition of long-acting beta-agonists to inhaled corticosteroids for chronic asthma in children

Comparison: 2 Long-acting beta2 agonist + ICS versus placebo + higher dose of ICS Outcome: 17 Linear growth

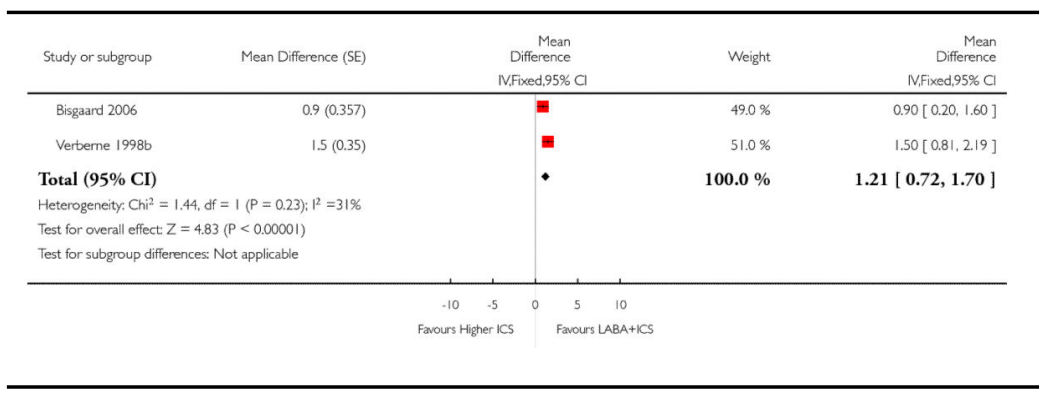




\section{Analysis 2.18}

Comparison 2 Long-acting beta 2 agonist + ICS versus placebo + higher dose of ICS, Outcome 18 \# nighttime rescue inhalations (puffs per day; mean over study period)

Review: Addition of long-acting beta-agonists to inhaled corticosteroids for chronic asthma in children

Comparison: 2 Long-acting beta2 agonist + ICS versus placebo + higher dose of ICS Outcome: 18 \# nighttime rescue inhalations (puffs per day; mean over study period)

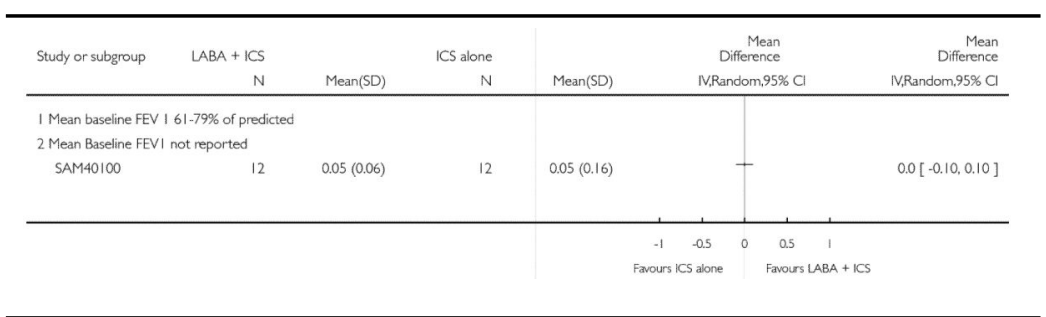

\section{Analysis 2.19}

Comparison 2 Long-acting beta 2 agonist + ICS versus placebo + higher dose of ICS, Outcome 19 \# daytime rescue inhalations (puffs per day; mean over study period)

Review: Addition of long-acting beta-agonists to inhaled corticosteroids for chronic asthma in children

Comparison: 2 Long-acting beta2 agonist + ICS versus placebo + higher dose of ICS Outcome: 19 \# daytime rescue inhalations (puffs per day; mean over study period)

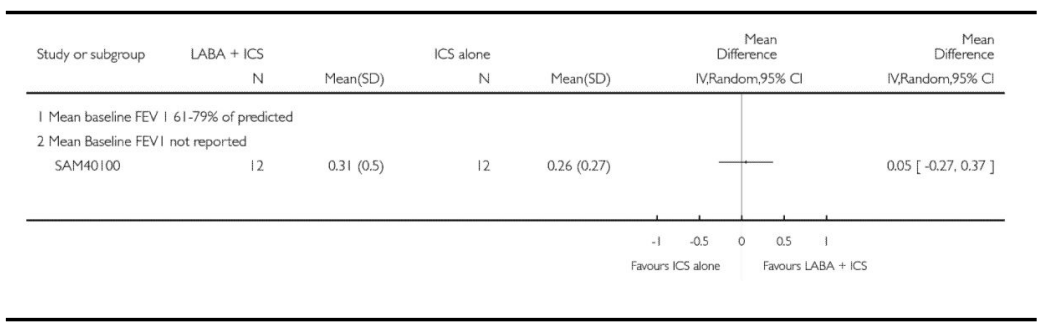




\section{Analysis 2.20}

\section{Comparison 2 Long-acting beta2 agonist + ICS versus} placebo + higher dose of ICS, Outcome 20 Deaths

Review: Addition of long-acting beta-agonists to inhaled corticosteroids for chronic asthma in children

Comparison: 2 Long-acting beta2 agonist + ICS versus placebo + higher dose of ICS Outcome: 20 Deaths

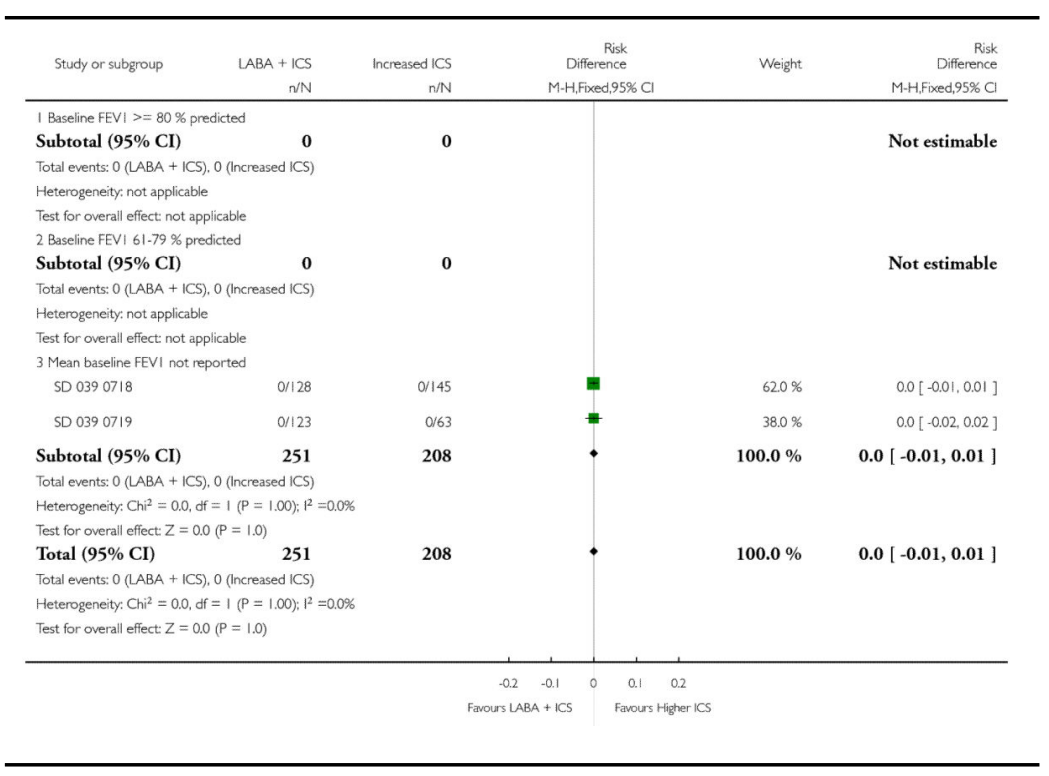

Analysis 2.21

Comparison 2 Long-acting beta2 agonist + ICS versus placebo + higher dose of ICS, Outcome 21 Daytime asthma symptom score (mean over study period)

Review: Addition of long-acting beta-agonists to inhaled corticosteroids for chronic asthma in children

Comparison: 2 Long-acting beta2 agonist + ICS versus placebo + higher dose of ICS Outcome: 21 Daytime asthma symptom score (mean over study period)

\begin{tabular}{|c|c|c|c|c|c|c|c|}
\hline Study or subgroup & $\begin{array}{r}\text { LABA + ICS } \\
N\end{array}$ & \multicolumn{3}{|c|}{ ICS alone } & \multicolumn{2}{|c|}{$\begin{array}{r}\begin{array}{r}\text { Std. } \\
\text { Mean } \\
\text { Difference }\end{array} \\
\text {. }\end{array}$} & $\begin{array}{r}\begin{array}{r}\text { Std. } \\
\text { Mean } \\
\text { Diference }\end{array} \\
\text { IV Fixed,95\% CI } \\
\end{array}$ \\
\hline \multirow{2}{*}{\multicolumn{8}{|c|}{$\begin{array}{l}\text { I Mean baseline FEVI }>>=80 \% \text { of predicted } \\
2 \text { Mean Baseline FEVI not reported }\end{array}$}} \\
\hline & & & & & & & \\
\hline \multirow[t]{2}{*}{ SAM40100 } & 12 & $0.46(0.5)$ & 12 & $0.44(0.6)$ & & & $0.03[-0.77,0.84]$ \\
\hline & & & & & $\begin{array}{ccc}-1 & -0.5 & 0 \\
\text { Farours treatrment }\end{array}$ & $\begin{array}{c}0.5 \quad \mid \\
\text { Faveurs control }\end{array}$ & \\
\hline
\end{tabular}


Analysis 2.22

Comparison 2 Long-acting beta2 agonist + ICS versus placebo + higher dose of ICS, Outcome 22 Nighttime asthma symptom score (mean over study period)

Review: Addition of long-acting beta-agonists to inhaled corticosteroids for chronic asthma in children

Comparison: 2 Long-acting beta2 agonist + ICS versus placebo + higher dose of ICS Outcome: 22 Nighttime asthma symptom score (mean over study period)

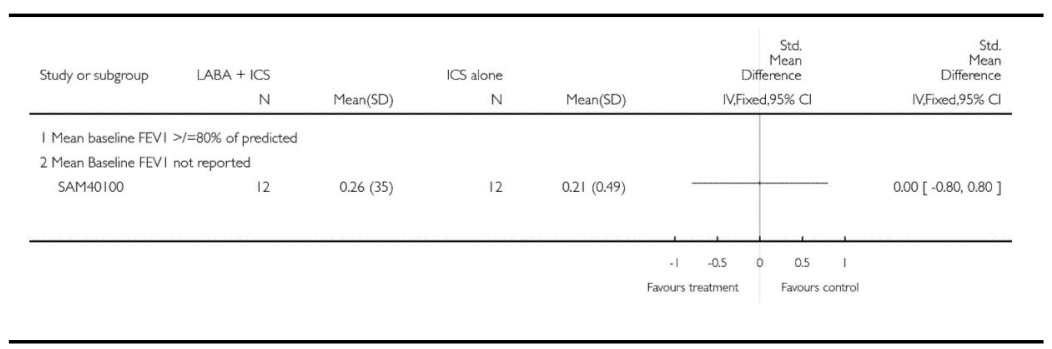




\section{Analysis 3.1}

Comparison 3 Subgroup analyses (comparison 01:

LABA+ICS versus SAME DOSE ICS),Outcome 1 \# patients with exacerbations requiring oral steroids by FEV1 \% predicted at baseline

Review: Addition of long-acting beta-agonists to inhaled corticosteroids for chronic asthma in children

Comparison: 3 Subgroup analyses (comparison 01: LABA+ICS versus SAME DOSE ICS) Outcome: 1 \# patients with exacerbations requiring oral steroids by FEV1 \% predicted at baseline

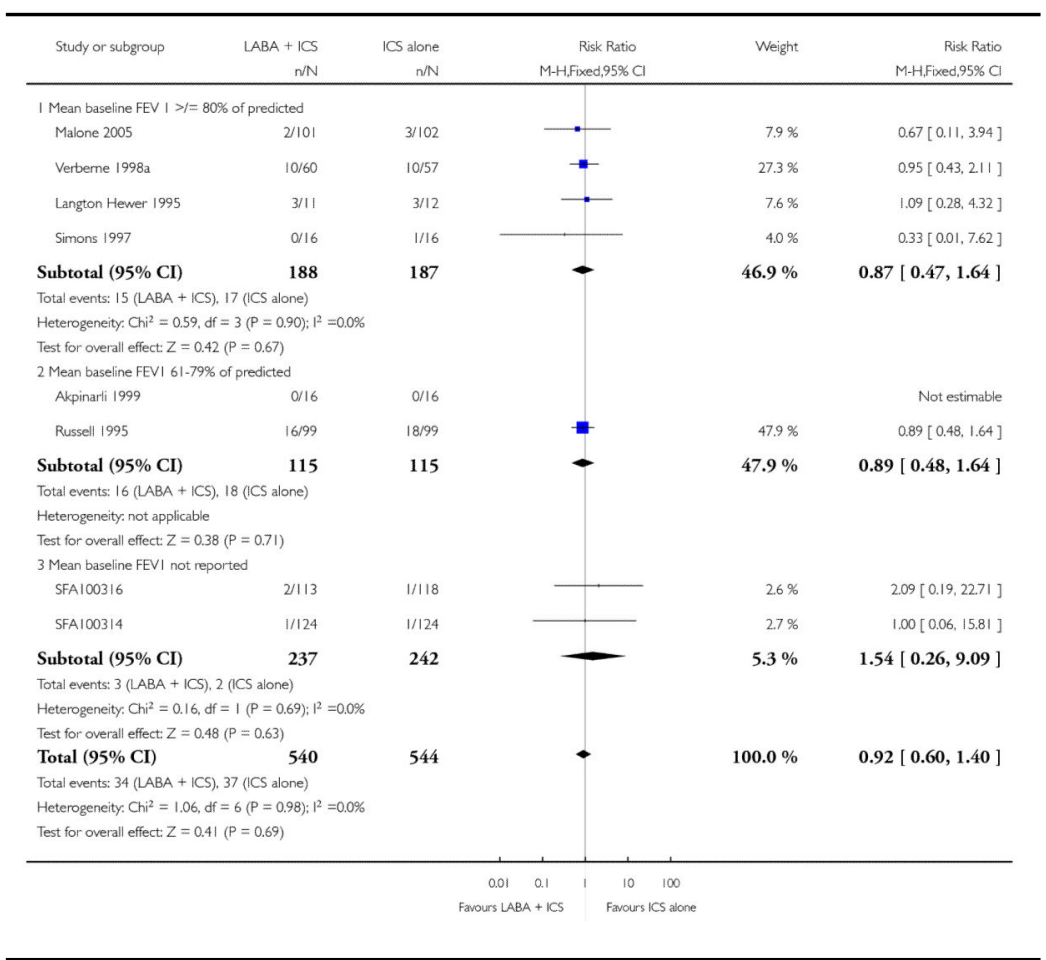


Analysis 3.2

Comparison 3 Subgroup analyses (comparison 01:

LABA+ICS versus SAME DOSE ICS), Outcome 2 \#

patients with exacerbations requiring oral steroids by whether funded by producers of LABA

Review: Addition of long-acting beta-agonists to inhaled corticosteroids for chronic asthma in children

Comparison: 3 Subgroup analyses (comparison 01: LABA+ICS versus SAME DOSE ICS) Outcome: 2 \# patients with exacerbations requiring oral steroids by whether funded by producers of LABA

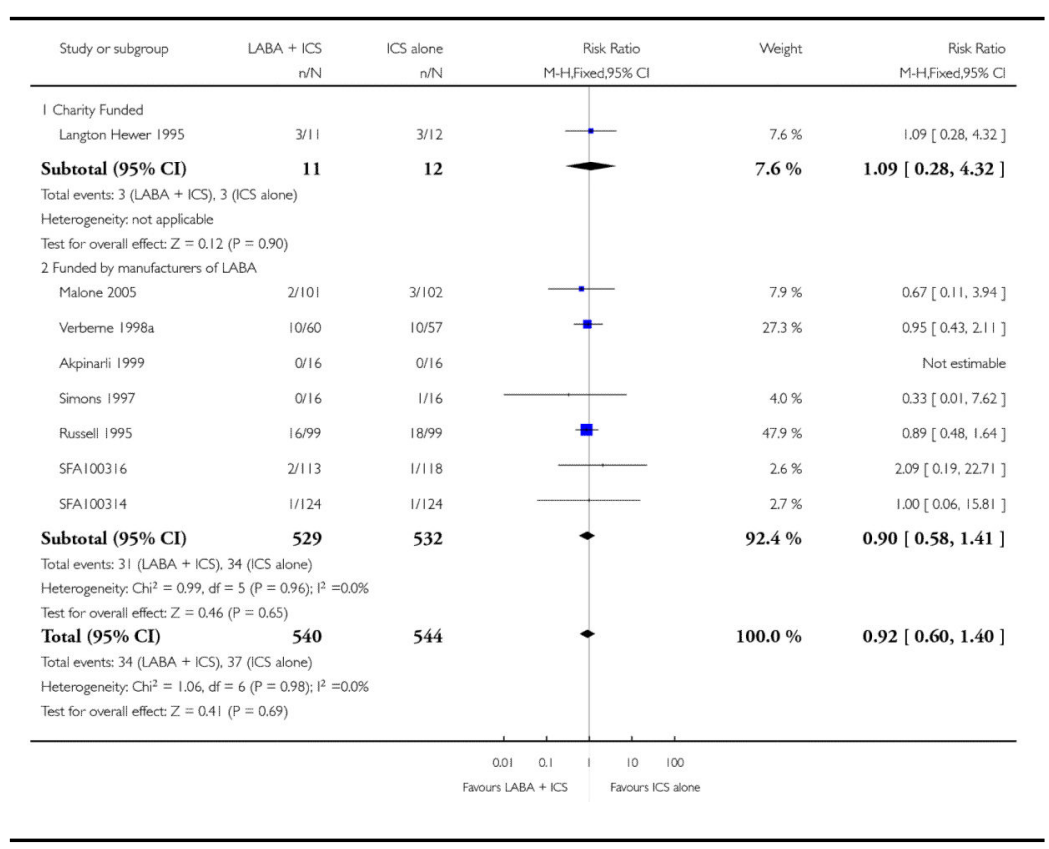


Analysis 3.3

Comparison 3 Subgroup analyses (comparison 01:

LABA+ICS versus SAME DOSE ICS),Outcome 3 \# patients with exacerbations requiring oral steroids by dose of ICS in both groups

Review: Addition of long-acting beta-agonists to inhaled corticosteroids for chronic asthma in children

Comparison: 3 Subgroup analyses (comparison 01: LABA+ICS versus SAME DOSE ICS) Outcome: 3 \# patients with exacerbations requiring oral steroids by dose of ICS in both groups

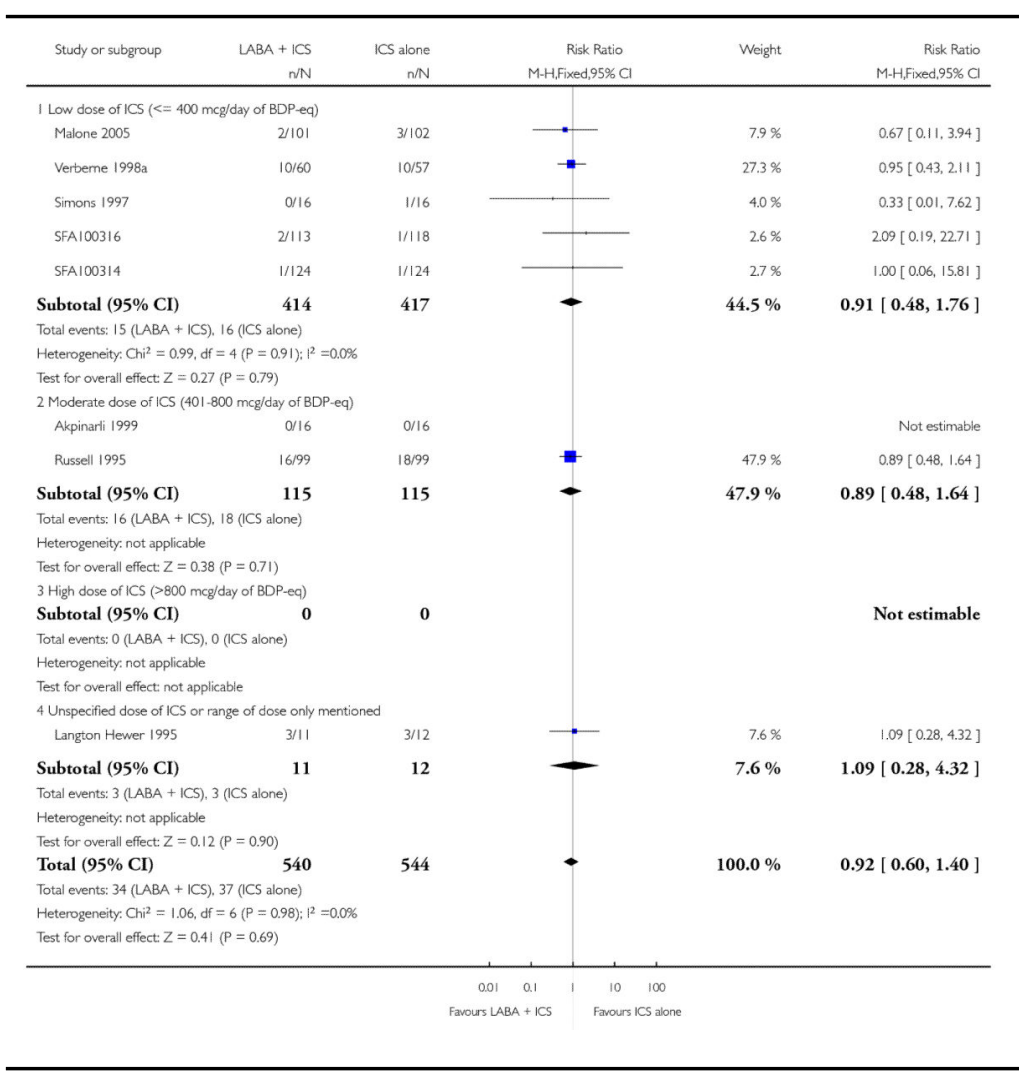


Analysis 3.4

Comparison 3 Subgroup analyses (comparison 01:

LABA+ICS versus SAME DOSE ICS),Outcome 4 \# patients with exacerbations requiring oral steroids by combination inhaler or separate inhaler for LABA

Review: Addition of long-acting beta-agonists to inhaled corticosteroids for chronic asthma in children

Comparison: 3 Subgroup analyses (comparison 01: LABA+ICS versus SAME DOSE ICS) Outcome: 4 \# patients with exacerbations requiring oral steroids by combination inhaler or separate inhaler for LABA

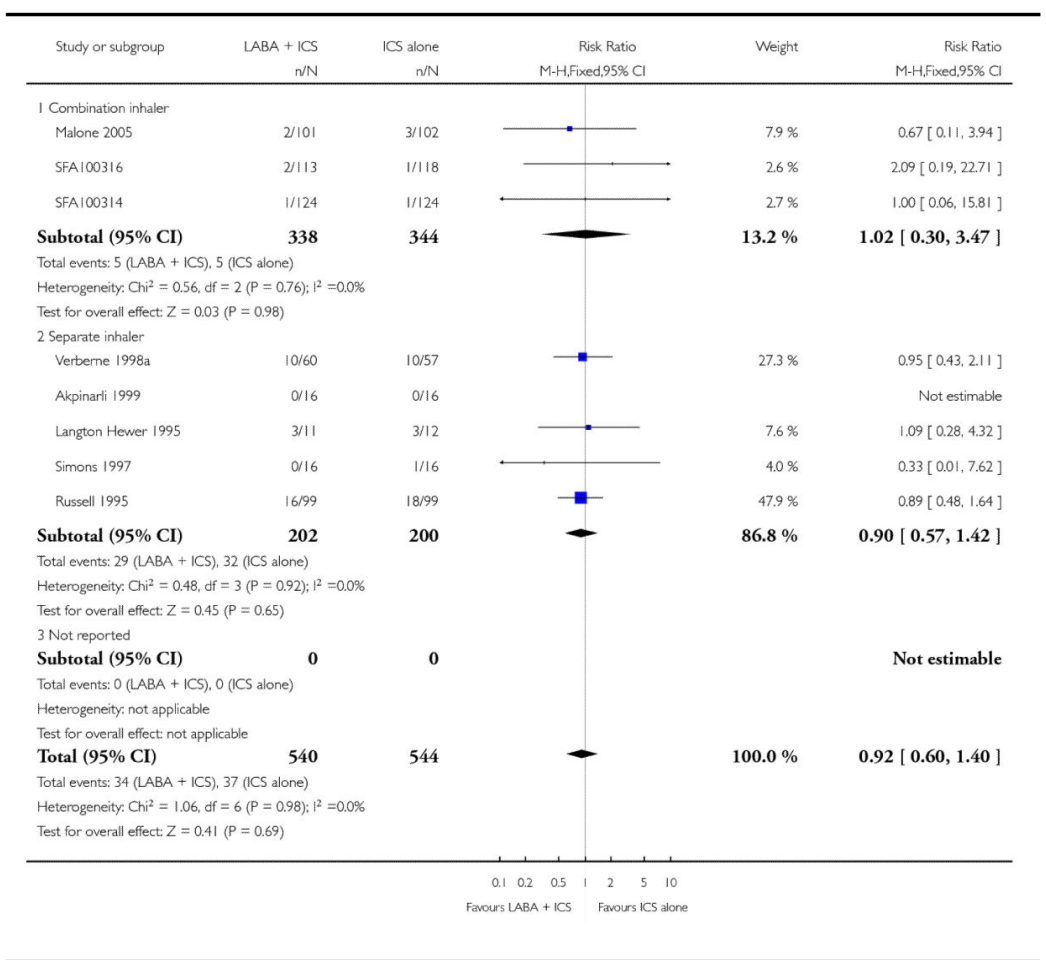




\section{Analysis 3.5}

Comparison 3 Subgroup analyses (comparison 01:

LABA+ICS versus SAME DOSE ICS), Outcome 5 \# patients with exacerbations requiring oral steroids by whether LABA dose is usual or higher than usual

Review: Addition of long-acting beta-agonists to inhaled corticosteroids for chronic asthma in children

Comparison: 3 Subgroup analyses (comparison 01: LABA+ICS versus SAME DOSE ICS) Outcome: 5 \# patients with exacerbations requiring oral steroids by whether LABA dose is usual or higher thanusual

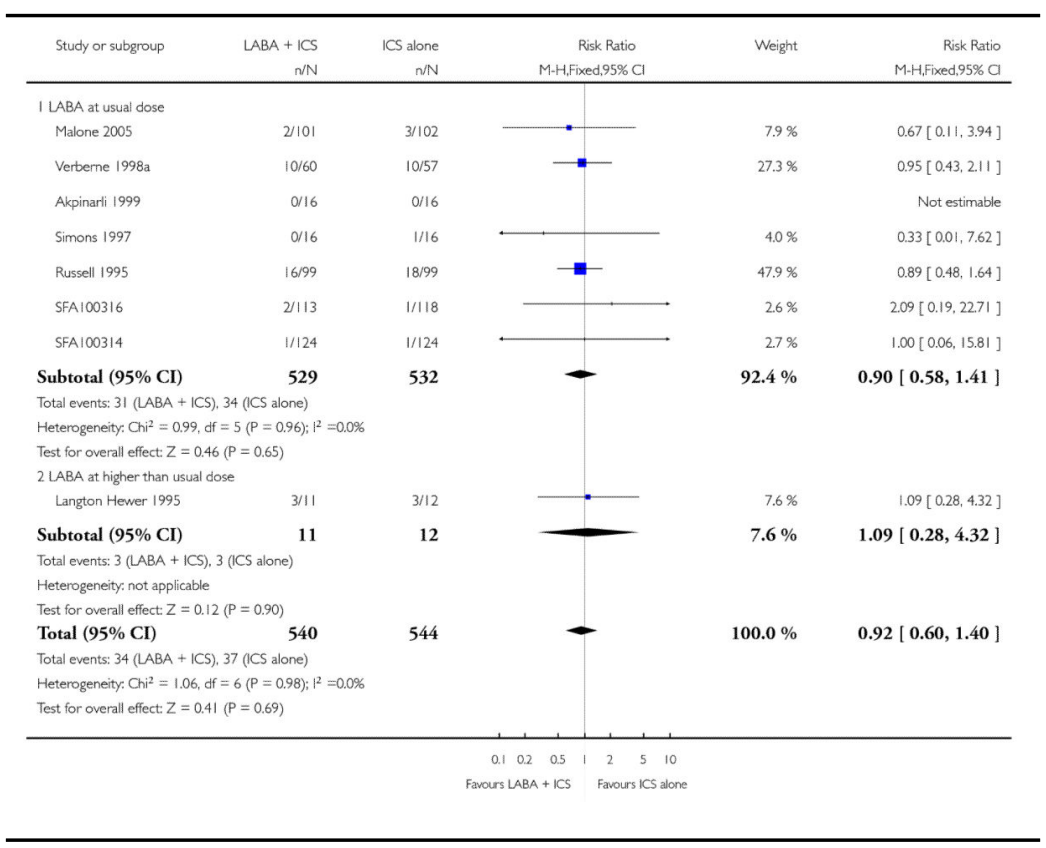


Analysis 3.6

Comparison 3 Subgroup analyses (comparison 01:

LABA+ICS versus SAME DOSE ICS),Outcome 6 \# patients with exacerbations requiring oral steroids by type of LABA

Review: Addition of long-acting beta-agonists to inhaled corticosteroids for chronic asthma in children

Comparison: 3 Subgroup analyses (comparison 01: LABA+ICS versus SAME DOSE ICS) Outcome: 6 \# patients with exacerbations requiring oral steroids by type of LABA

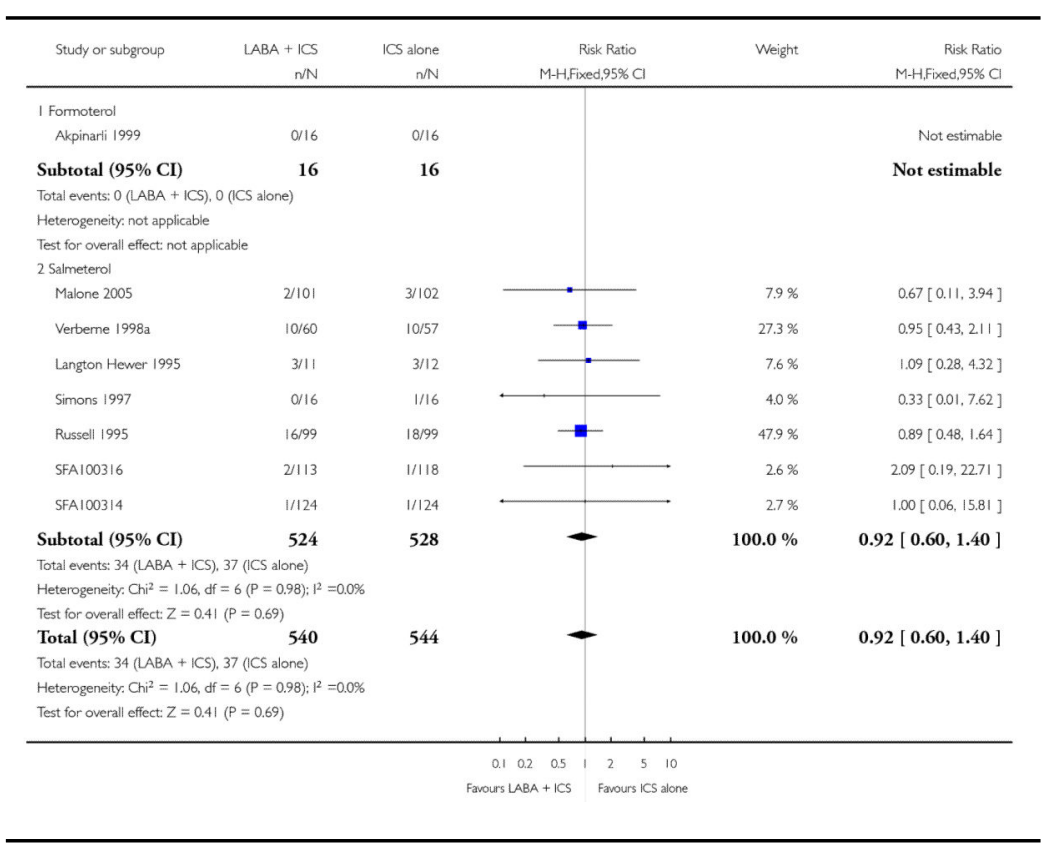




\section{Analysis 3.7}

Comparison 3 Subgroup analyses (comparison 01:

LABA+ICS versus SAME DOSE ICS),Outcome 7 \#

patients with exacerbations requiring oral steroids by

trial duration

Review: Addition of long-acting beta-agonists to inhaled corticosteroids for chronic asthma in children

Comparison: 3 Subgroup analyses (comparison 01: LABA+ICS versus SAME DOSE ICS) Outcome: 7 \# patients with exacerbations requiring oral steroids by trial duration

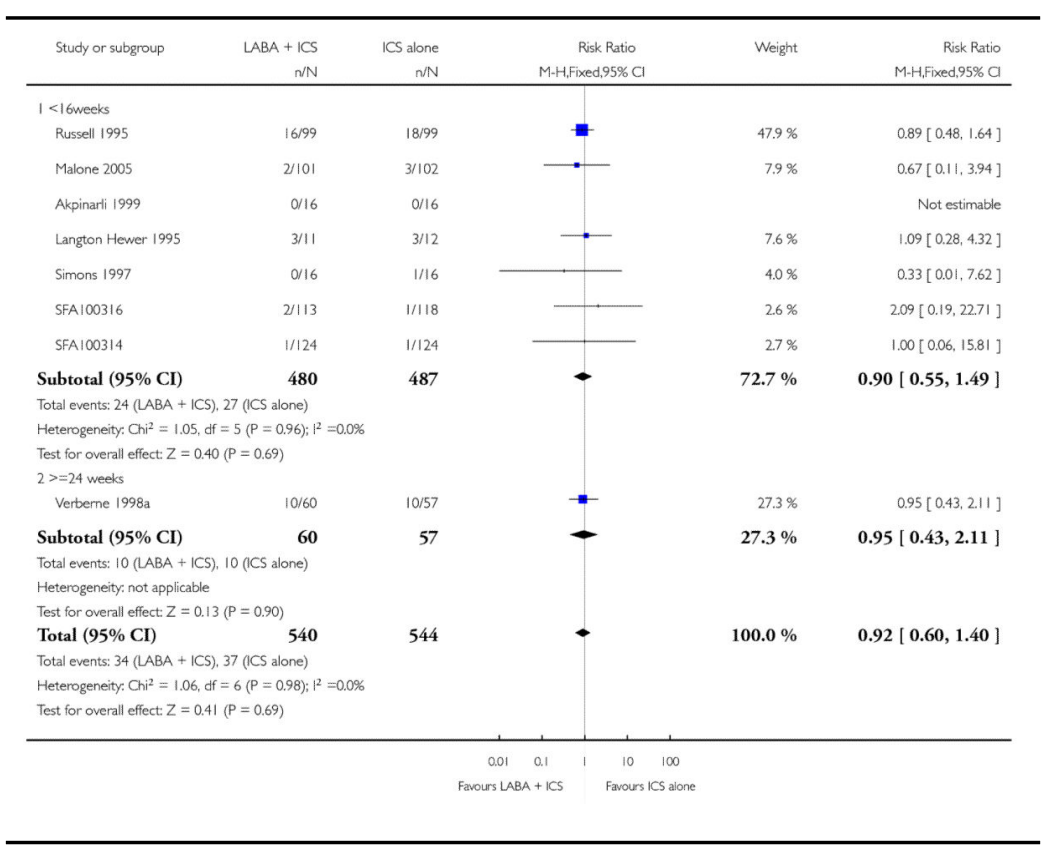




\section{Analysis 3.8}

Comparison 3 Subgroup analyses (comparison 01:

LABA+ICS versus SAME DOSE ICS), Outcome 8

Change in FEV1 at endpoint ( $\mathrm{L}$ or \% predicted)

stratifying by type of LABA used

Review: Addition of long-acting beta-agonists to inhaled corticosteroids for chronic asthma in children

Comparison: 3 Subgroup analyses (comparison 01: LABA+ICS versus SAME DOSE ICS) Outcome: 8 Change in FEV1 at endpoint (L or \% predicted) stratifying by type of LABA used

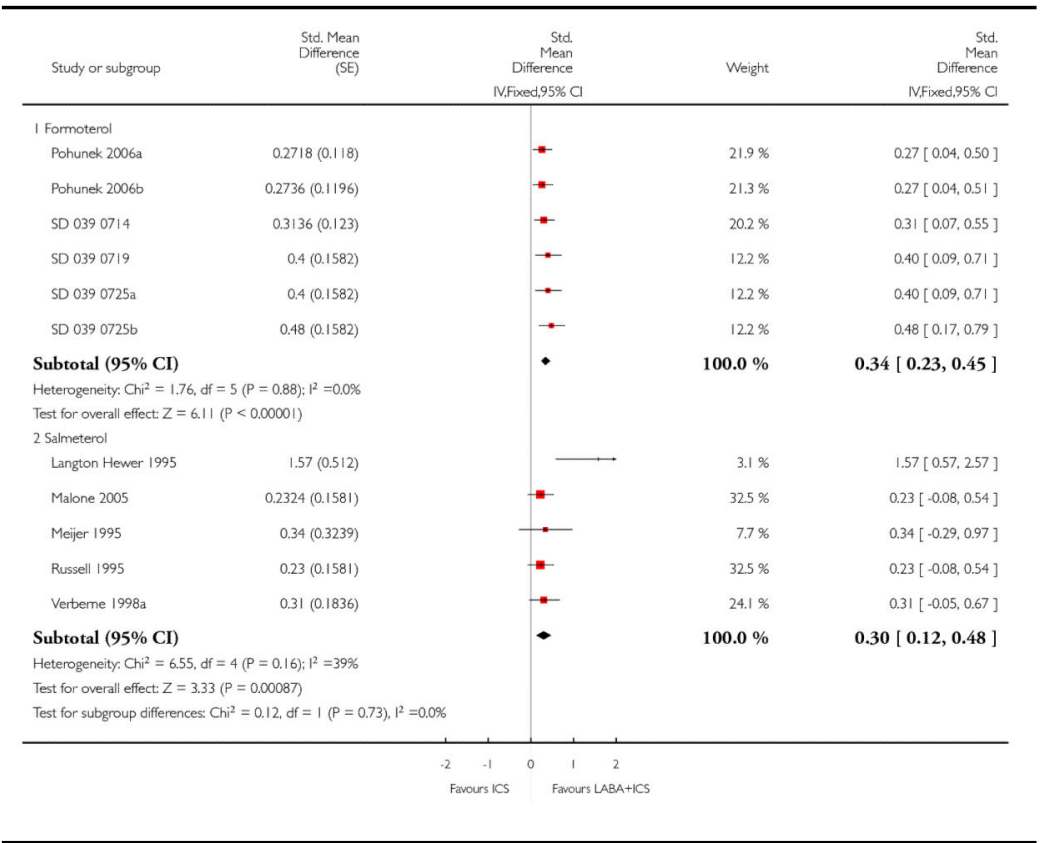


Analysis 4.1

Comparison 4 Subgroup analyses (comparison 02:

LABA+ICS versus HIGHER DOSE ICS), Outcome 1 \# patients with exacerbations requiring oral steroids by

FEV1 \% predicted at baseline

Review: Addition of long-acting beta-agonists to inhaled corticosteroids for chronic asthma in children

Comparison: 4 Subgroup analyses (comparison 02: LABA+ICS versus HIGHER DOSE ICS)

Outcome: 1 \# patients with exacerbations requiring oral steroids by FEV1 \% predicted at baseline

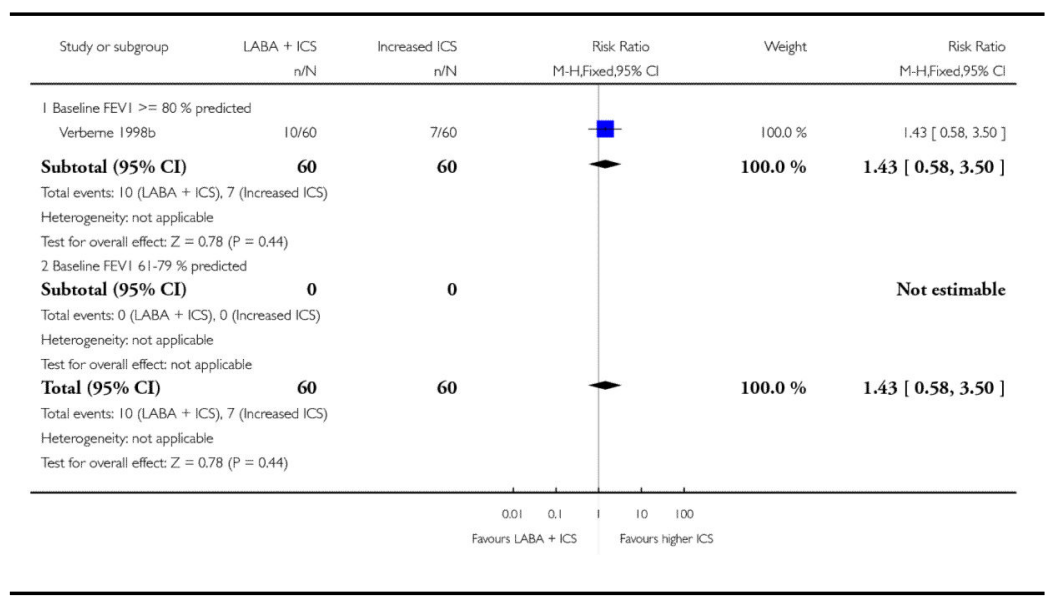




\section{Analysis 4.2}

Comparison 4 Subgroup analyses (comparison 02:

LABA+ICS versus HIGHER DOSE ICS), Outcome 2 \# patients with exacerbations requiring oral steroids by whether funded by producers of LABA

Review: Addition of long-acting beta-agonists to inhaled corticosteroids for chronic asthma in children

Comparison: 4 Subgroup analyses (comparison 02: LABA+ICS versus HIGHER DOSE ICS)

Outcome: 2 \# patients with exacerbations requiring oral steroids by whether funded by producers of LABA

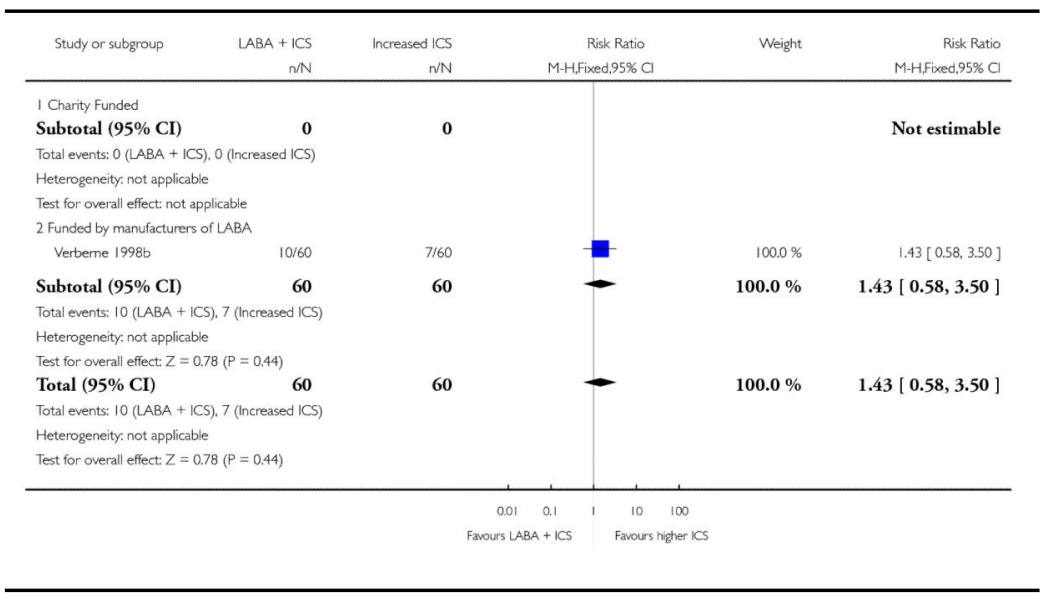


Analysis 4.3

Comparison 4 Subgroup analyses (comparison 02:

LABA+ICS versus HIGHER DOSE ICS), Outcome 3 \# patients with exacerbations requiring oral steroids by dose of ICS in control groups

Review: Addition of long-acting beta-agonists to inhaled corticosteroids for chronic asthma in children

Comparison: 4 Subgroup analyses (comparison 02: LABA+ICS versus HIGHER DOSE ICS)

Outcome: 3 \# patients with exacerbations requiring oral steroids by dose of ICS in control groups

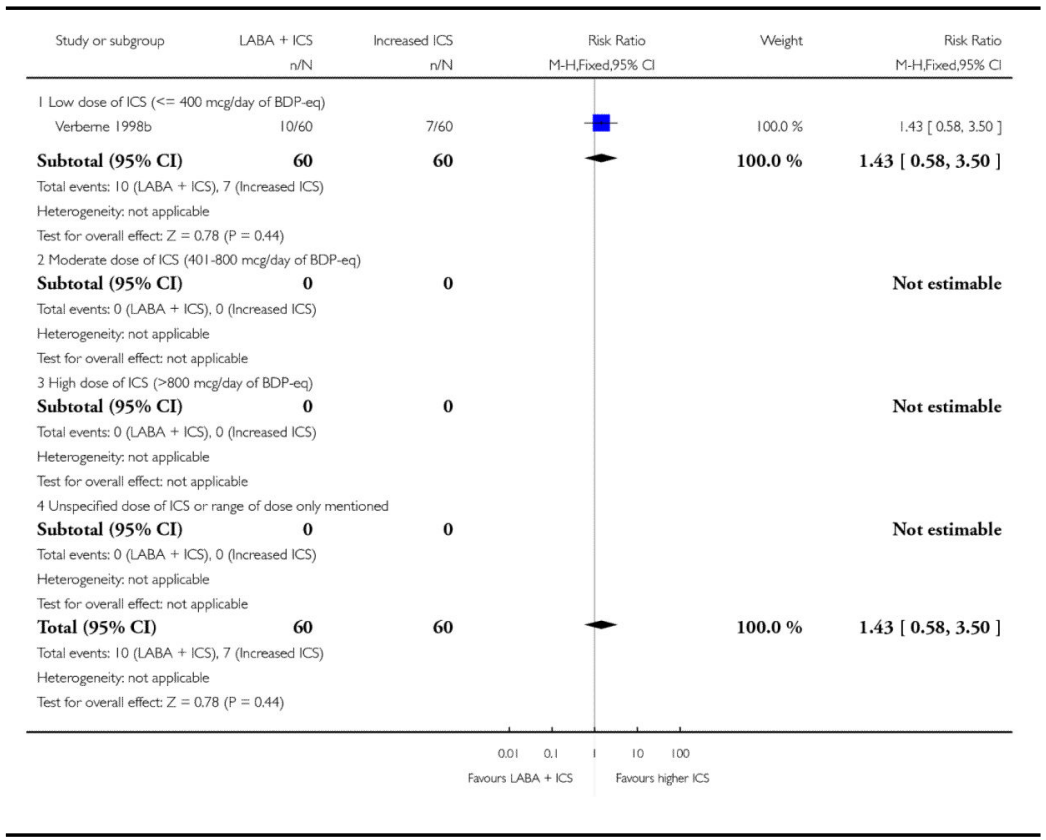


Analysis 4.4

Comparison 4 Subgroup analyses (comparison 02:

LABA+ICS versus HIGHER DOSE ICS), Outcome 4 \# patients with exacerbations requiring oral steroids by combination inhaler or separate inhaler for LABA

Review: Addition of long-acting beta-agonists to inhaled corticosteroids for chronic asthma in children

Comparison: 4 Subgroup analyses (comparison 02: LABA+ICS versus HIGHER DOSE ICS)

Outcome: 4 \# patients with exacerbations requiring oral steroids by combination inhaler or separate inhaler for LABA

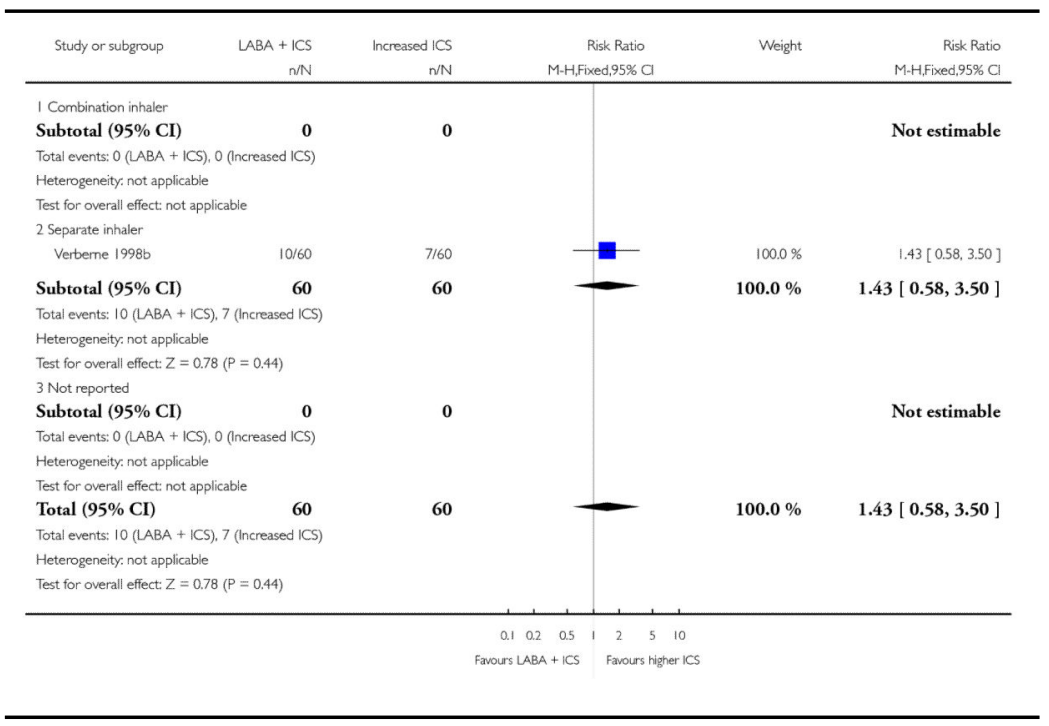


Analysis 4.5

Comparison 4 Subgroup analyses (comparison 02:

LABA+ICS versus HIGHER DOSE ICS), Outcome 5 \# patients with exacerbations requiring oral steroids by whether LABA dose is usual or higher than usual

Review: Addition of long-acting beta-agonists to inhaled corticosteroids for chronic asthma in children

Comparison: 4 Subgroup analyses (comparison 02: LABA+ICS versus HIGHER DOSE ICS)

Outcome: 5 \# patients with exacerbations requiring oral steroids by whether LABA dose is usual or higher thanusual

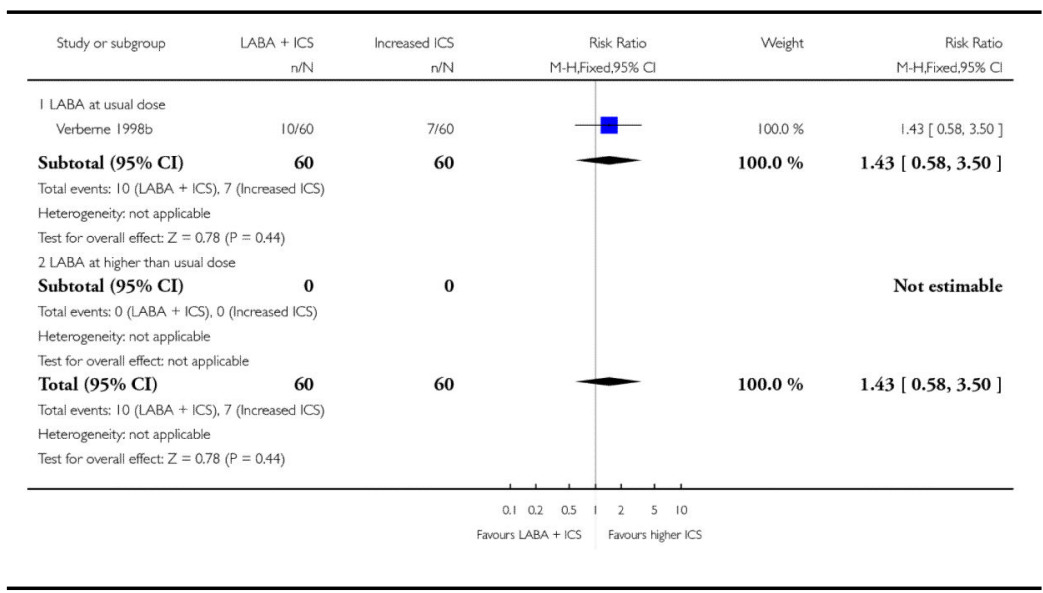


Analysis 4.6

Comparison 4 Subgroup analyses (comparison 02:

LABA+ICS versus HIGHER DOSE ICS), Outcome 6 \# patients with exacerbations requiring oral steroids by type of LABA

Review: Addition of long-acting beta-agonists to inhaled corticosteroids for chronic asthma in children

Comparison: 4 Subgroup analyses (comparison 02: LABA+ICS versus HIGHER DOSE ICS)

Outcome: 6 \# patients with exacerbations requiring oral steroids by type of LABA

\begin{tabular}{|c|c|c|c|c|c|}
\hline Study or subgroup & $\begin{array}{r}L A B A+I C S \\
n / N \\
\end{array}$ & $\begin{array}{r}\text { Increased ICS } \\
n / N\end{array}$ & $\begin{array}{l}\text { Risk Ratio } \\
\text { M-H,Fixed,95\% CI }\end{array}$ & Weight & $\begin{array}{r}\text { Risk Ratio } \\
\text { M-H.Fxed,95\% C }\end{array}$ \\
\hline \multicolumn{6}{|l|}{ | Formoterol } \\
\hline Subtotal $(95 \% \mathrm{CI})$ & 0 & 0 & & & Not estimable \\
\hline \multicolumn{6}{|c|}{ Total events: $0($ LABA + ICS), $0($ (ncreased ICS $)$} \\
\hline \multicolumn{6}{|c|}{ Heterogeneity. not applicable } \\
\hline \multicolumn{6}{|c|}{ Test for overal effect: not applicable } \\
\hline \multicolumn{6}{|c|}{2 Salmeterol } \\
\hline Verberne $1998 \mathrm{~b}$ & 10/60 & $7 / 60$ & $=$ & $100.0 \%$ & $1.43[0.58,3.50]$ \\
\hline Subtotal $(95 \% \mathrm{CI})$ & 60 & 60 & $\longrightarrow$ & $100.0 \%$ & $1.43[0.58,3.50]$ \\
\hline \multicolumn{6}{|c|}{ Total events: 10 (LABA + ICS), 7 (Increased ICS) } \\
\hline \multicolumn{6}{|c|}{ Heterogeneity not applicable } \\
\hline \multicolumn{6}{|c|}{ Test for overall effect: $Z=0.78(P=0.44)$} \\
\hline Total $(95 \% \mathrm{CI})$ & 60 & 60 & - & $100.0 \%$ & $1.43[0.58,3.50]$ \\
\hline \multicolumn{6}{|c|}{ Total events: 10 (LABA + ICSS), 7 (Increased ICS) } \\
\hline \multicolumn{6}{|c|}{ Heterogeneity, not applicable } \\
\hline \multicolumn{6}{|c|}{ Test for overall effect: $Z=0.78(P=0.44)$} \\
\hline & & & 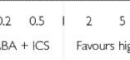 & & \\
\hline
\end{tabular}


Analysis 4.7

Comparison 4 Subgroup analyses (comparison 02:

LABA+ICS versus HIGHER DOSE ICS), Outcome 7 \# patients with exacerbations requiring oral steroids by trial duration

Review: Addition of long-acting beta-agonists to inhaled corticosteroids for chronic asthma in children

Comparison: 4 Subgroup analyses (comparison 02: LABA+ICS versus HIGHER DOSE ICS)

Outcome: 7 \# patients with exacerbations requiring oral steroids by trial duration

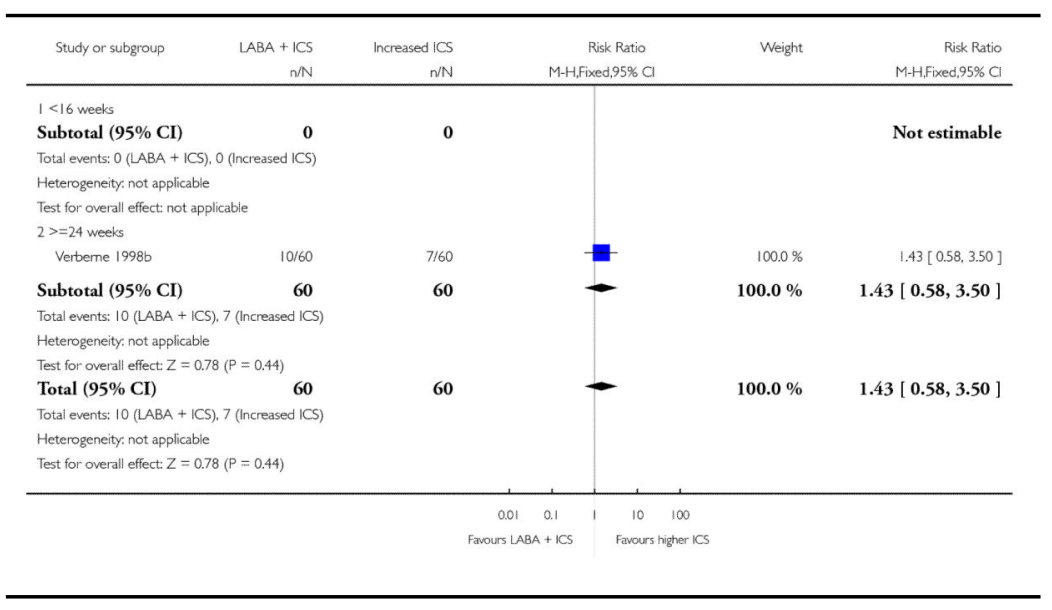

\section{Analysis 5.1}

Comparison 5 WMD archive, Outcome 1 Change in FEV1 (\% predicted) at endpoint

Review: Addition of long-acting beta-agonists to inhaled corticosteroids for chronic asthma in children

Comparison: 5 WMD archive

Outcome: 1 Change in FEV1 (\% predicted) at endpoint

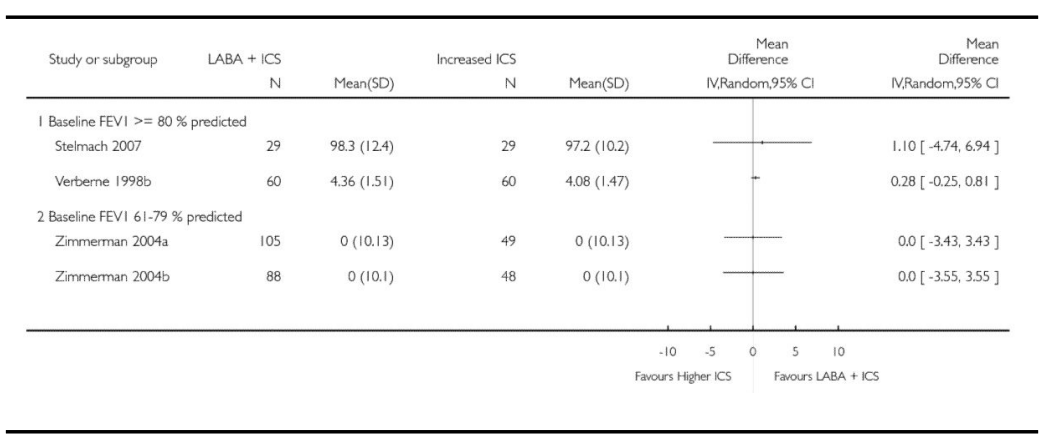


Analysis 5.2

Comparison 5 WMD archive, Outcome 2 Change in height at 1 year

Review: Addition of long-acting beta-agonists to inhaled corticosteroids for chronic asthma in children

Comparison: 5 WMD archive

Outcome: 2 Change in height at 1 year

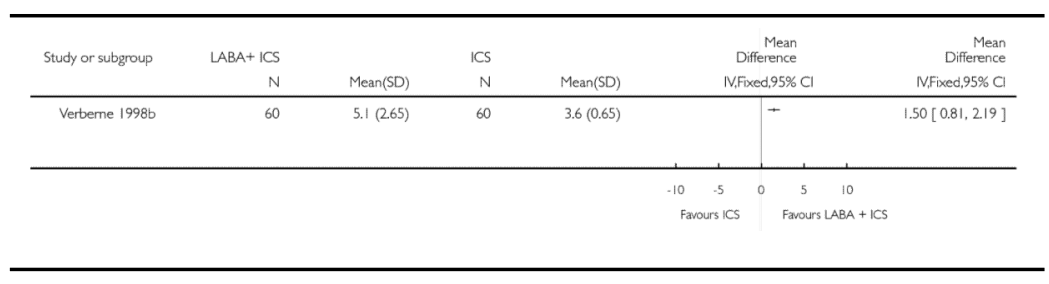

Analysis 5.3

Comparison 5 WMD archive, Outcome 3 Change in am PEF

Review: Addition of long-acting beta-agonists to inhaled corticosteroids for chronic asthma in children

Comparison: 5 WMD archive

Outcome: 3 Change in am PEF

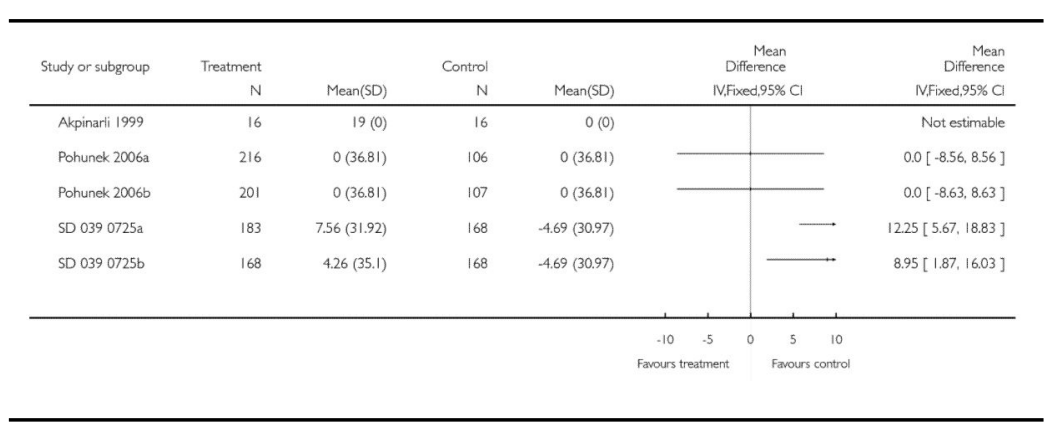


Analysis 5.6

\section{Comparison 5 WMD archive, Outcome 6 Pm PEF}

Review: Addition of long-acting beta-agonists to inhaled corticosteroids for chronic asthma in children

Comparison: 5 WMD archive

Outcome: 6 Pm PEF

\begin{tabular}{|c|c|c|c|c|c|c|c|}
\hline \multirow{3}{*}{$\begin{array}{l}\text { Study or subgroup } \\
\text { Morice 2008a }\end{array}$} & \multirow{3}{*}{$\begin{array}{r}\text { Treatment } \\
\mathrm{N} \\
212\end{array}$} & \multicolumn{3}{|c|}{ Control } & \multirow{2}{*}{\multicolumn{2}{|c|}{$\begin{array}{c}\text { Mean } \\
\text { Difference } \\
\text { IV,Fixed,95\% C }\end{array}$}} & \multirow{3}{*}{$\begin{array}{r}\begin{array}{r}\text { Mean } \\
\text { Difference }\end{array} \\
\text { INFixed,95\% a } \\
0.0[-6.62,6.62]\end{array}$} \\
\hline & & \multirow{2}{*}{$\begin{array}{r}\operatorname{Mean}(\mathrm{SD}) \\
0(28.2)\end{array}$} & \multirow{2}{*}{$\frac{\mathrm{N}}{104}$} & \multirow{2}{*}{$\begin{array}{r}\text { Mean(SD) } \\
0(28.2)\end{array}$} & & & \\
\hline & & & & & 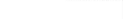 & - & \\
\hline SD 0390718 & 128 & $21.4(40.62)$ & 145 & $5.79(35.3)$ & & $\longrightarrow$ & $15.61[6.53,24.69]$ \\
\hline & & & & & $\begin{array}{cc}-10 & -5 \\
\text { Farours treatment }\end{array}$ & $\begin{array}{ccc}0 & 5 & 10 \\
& \text { Farours control }\end{array}$ & \\
\hline
\end{tabular}

Analysis 5.7

Comparison 5 WMD archive, Outcome 7 Change in pm PEF

Review: Addition of long-acting beta-agonists to inhaled corticosteroids for chronic asthma in children

Comparison: 5 WMD archive

Outcome: 7 Change in pm PEF

\begin{tabular}{|c|c|c|c|c|c|c|}
\hline \multirow[t]{2}{*}{ Study or subgroup } & \multirow{2}{*}{$\begin{array}{r}\text { Treatment } \\
\mathrm{N}\end{array}$} & \multicolumn{3}{|c|}{ Control } & $\begin{array}{r}\text { Mean } \\
\text { Difference }\end{array}$ & \multirow{2}{*}{$\begin{array}{r}\text { Mean } \\
\text { Difference } \\
\text { IV.Fixed,95\% C }\end{array}$} \\
\hline & & $\operatorname{Mean}(S D)$ & N & $\operatorname{Mean}(\mathrm{SD})$ & IN,Fixed,95\% C & \\
\hline SD 039 0725a & 183 & $6.69(35.52)$ & 84 & $-5.84(29.89)$ & $\rightarrow$ & $12.53[4.32,20.74]$ \\
\hline \multirow[t]{2}{*}{ SD $0390725 b$} & 168 & $0.45(34.92)$ & 84 & $-5.84(29.89)$ & - & $6.29[-2.00,14.58]$ \\
\hline & & & & & $\begin{array}{cccr}-100 & -50 & 0 & 50 \\
\text { Favours treatment } & & \text { Favo } \\
\end{array}$ & \\
\hline
\end{tabular}


Analysis 5.8

Comparison 5 WMD archive, Outcome 8 Change in

FEV1 (L) versus baseline

Review: Addition of long-acting beta-agonists to inhaled corticosteroids for chronic asthma in children

Comparison: 5 WMD archive

Outcome: 8 Change in FEV1 (L) versus baseline

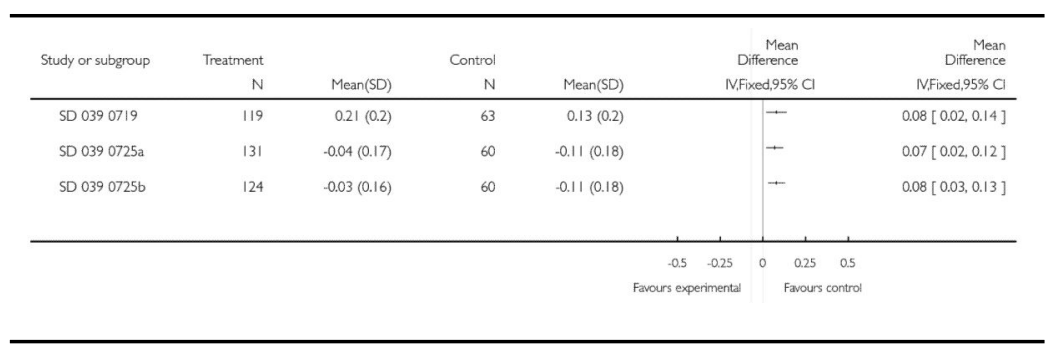

Analysis 5.9

Comparison 5 WMD archive, Outcome 9 Change in evening PEF ( $\mathrm{L} / \mathrm{min})$ at endpoint

Review: Addition of long-acting beta-agonists to inhaled corticosteroids for chronic asthma in children

Comparison: 5 WMD archive

Outcome: 9 Change in evening PEF (L/min) at endpoint

\begin{tabular}{|c|c|c|c|c|c|c|c|}
\hline \multirow{2}{*}{$\begin{array}{l}\text { Study or subgroup } \\
\text { | Baseline FEV| >= }\end{array}$} & $\begin{array}{r}\angle A B A+I C S \\
N\end{array}$ & \multicolumn{3}{|c|}{ ICS alone } & \multicolumn{2}{|c|}{$\begin{array}{c}\text { Mean } \\
\text { Difference }\end{array}$} & $\begin{array}{r}\text { Mean } \\
\text { Difference } \\
\text { IV,Fixed,95\% Cl } \\
\end{array}$ \\
\hline & \multicolumn{5}{|c|}{2 Baseline FEVI $61.79 \%$ preditted } & & \\
\hline Bisgaard 2006 & 117 & $22(34.51)$ & 106 & $15(34.51)$ & & + & $7.00[-207,16.07]$ \\
\hline \multicolumn{8}{|c|}{3 Mean Baseline FEVI not reported } \\
\hline SAM40012b & 176 & $43.2(41.13)$ & 180 & $38.8(40.2)$ & & + & $4.40[-4.05,12.85]$ \\
\hline & & & & & $\begin{array}{cc}-100 & -50 \\
\text { Favours ICS alone }\end{array}$ & $\begin{array}{r}\text { so } \\
\text { Favour }\end{array}$ & \\
\hline
\end{tabular}

\section{Appendix 1. Randomisation procedures for GSK sponsored studies}

The procedures for randomising GSK sponsored studies has been detailed in correspondence between Richard Follows and TL, the details of which are given below:

The randomisation software is a computer-generated, centralised programme (RandAll). After verification that the randomisation sequence is suitable for the study design (crossover, block or stratification), Clinical Supplies then package the treatments according the randomisation list generated. Concealment of allocation is maintained by a third party, since the sites phone in and are allocated treatments on that basis. Alternatively a third party may dispense the drug at the sites. Unblinding of data for interim analyses can only be done 
through RandAll, and are restricted so that only those reviewing the data are unblinded to treatment group allocation.

\section{WHAT'S NEW}

Last assessed as up-to-date: 8 December 2008.

\begin{tabular}{lll}
\hline Date & Event & Description \\
\hline 8 December 2009 & Amended & $\begin{array}{l}\text { We have revised the reporting of correspondence in relation to missing data } \\
\text { The correspondence regarding the data from Bisgaard 2006 was made directly with } \\
\text { the sponsors, not with Hans Bisgaard. The sponsors were unable to provide data on } \\
\text { children with exacerbations requiring oral corticosteroids from this study }\end{array}$ \\
\hline
\end{tabular}

\section{HISTORY}

Review first published: Issue 3, 2009

\begin{tabular}{lll}
\hline Date & Event & Description \\
\hline 21 April 2008 & Amended & Converted to new review format. \\
\hline
\end{tabular}

\section{DIFFERENCES BETWEEN PROTOCOL AND REVIEW}

The principal difference between the protocol for the set of reviews relating to long-acting beta-agonists and inhaled steroids and this review is the risk of bias assessment. This has been developed by methodologists and statisticians and aims to provide a transparent mechanism for reporting the design of clinical trials, and the extent to which review authors judge them to be at risk of bias.

\section{References to studies included in this review}

Akpinarli 1999 \{published data only\} . Akpinarli A, Tuncer A, Saraclar Y, Sekerel BE, Kalayci O. Effect of formoterol on clinical parameters and lung functions in patients with bronchial asthma: a randomised controlled trial. Archives of Disease in Childhood. 1999; 81(1):45-8. [PubMed: 10373134]

Bisgaard 2006 \{published data only\} . Barnes PJ, O’Byrne PM, Rodriguez-Roisin R, Runnerstrom E, Sandstrom T, Svensson K, Tattersfield A. Oxis and Pulmicort turbuhaler in the management of asthma OPTIMA international study group. Treatment of mild persistent asthma with low doses of inhaled Budesonide alone or in combination with Formoterol. Thorax. 2000; 55(Suppl 3):A4.

Barnes PJ, O’Byrne PM, Rodriguez-Roisin R, Runnerstrom E, Sandstrom T, Svensson K, Tattersfield A. Treatment of mild persistent asthma with low doses of inhaled budesonide alone or in combination with formoterol. For the Oxis and Pulmicort Turbuhaler in the Management of Asthma (OPTIMA) international study group. Thorax. 2000; 55(Suppl 3):s5.

Bisgaard H, Hultquist C. Budesonide/formoterol for maintenance and as needed - a new approach to asthma management in children [Abstract]. European Respiratory Journal. 2005; 26(Suppl 49) Abstract No. 1060.

*Bisgaard H, Le Roux P, Bjamer D, Dymek A, Vermeulen JH, Hultquist C. Budesonide/formoterol maintenance plus reliever therapy - a new strategy in pediatric asthma. Chest. 2006; 130(6) 
Jönsson BG, Berggren FE, Svensson K, O'Byrne PM. Budesonide and formoterol in mild persistent asthma compared with doubling the dose of budesonide - a cost-effectiveness analysis. European Respiratory Journal. 2001; 18(Suppl 33):517s.

Jönsson BG, Berggren FE, Svensson K, O’Byrne PM. Economic results of adding formoterol to budesonide in mild persistent asthma. European Respiratory Journal. 2001; 18(Suppl 33):331s.

O'Byrne PM. Acute asthma intervention: Insights from the STAY study. Journal of Allergy \& Clinical Immunology. 2007; 119(6):1332-6. [PubMed: 17451803]

O’Byrne PM, Barnes PJ, Rodriguez-Roisin R, Sandtröm T, Tattersfield AE, Runnerström EM, Svensson K. Addition of formoterol Turbuhaler® to budesonide Tubuhaler ${ }^{\circledR}$ is safe and well tolerated in the long-term treatment of mild asthma: results from the OPTIMA trial. European Respiratory Journal. 2001; 18(Suppl 33):330s.

O’Byrne PM, Bisgaard H, Godard PP, Pistolesi M, Palmqvist M, Zhu Y, Ekstrom T, Bateman ED. Budesonide/formoterol combination therapy as both maintenance and reliever medication in asthma.[see comment]. American Journal of Respiratory \& Critical Care Medicine. 2005; 171(2): 129-36. [PubMed: 15502112]

SD-039-0673. Efficacy and safety of budesonide/formoterol (Symbicort) Turbuhaler ${ }^{\circledR}$ as Single Therapy in patients with mild-moderate asthma. Comparison with Symbicort Turbuhaler and Pulmicort ${ }^{\circledR}$ Turbuhaler as maintenance therapy, both complemented with Bricanyl ${ }^{\circledR}$ Turbuhaler (STAY). 2006 http://www.astrazenecaclinicaltrials.com.

Heuck 2000 \{published data only\} . Heuck C, Heickendorff L, Wolthers OD. A randomized controlled trial of short term growth and collagen turnover in asthmatics with inhaled formoterol and budesonide. Archives of Disease of Childhood. 2000; 83(4):334-9.

Heuck, C.; Heickendorff, L.; Wolthers, OD.; Sygehus, S. Short term growth and collagen turnover in asthmatics treated inhaled formoterol and budesonide. European Respiratory Society; Madrid, Spain: Oct 9-13. 1999 p. 3641999

Langton Hewer 1995 \{published data only\} . Langton Hewer S, Hobbs J, French D, Lenney W. Pilgrims progress: the effect of salmeterol in older children with chronic severe asthma. Respiratory Medicine. 1995; 89(6):435-40. [PubMed: 7644775]

Malone 2005 \{published and unpublished data\} . Dorinsky P, Emmett A, Sutton L. Reduced risk for asthma exacerbations in pediatric patients receiving salmeterol plus inhaled corticosteroids (ICS) vs ICS alone [Abstract]. European Respiratory Journal. 2004; 24(Suppl 48):308s.

House K, Dorinsky PM, Stauffer J, Schoaf L, Ellsworth A. The Safety of Fluticasone propionate/ Salmeterol Diskus(R) in Pediatric Patients Ages 4-11 with Asthma [Abstract]. Chest. 2004; 126(Suppl 4):911S.

*Malone R, LaForce C, Nimmagadda S, Schoaf L, House K, Ellsworth A, Dorinsky P. The safety of twice-daily treatment with fluticasone propionate and salmeterol in pediatric patients with persistent asthma. Annals of Allergy, Asthma, \& Immunology. 2005; 95(1):66-71.

SAS30031. A Randomized, Double-Blind, 12-Week Trial Evaluating the Safety of the Fluticasone Propionate/SalmeterolDISKUS Combination Product 100/50mcg BID Versus Fluticasone Propionate DISKUS 100mcg BID in SymptomaticPediatric Subjects (4-11 Years) With Asthma. 2005 http://www.ctr.gsk.co.uk.

Scott C, Wu W, Ellsworth A, Crim C. Efficacy and safety of fluticasone propionate/salmeterol DISKUS and fluticasone propionate DISKUS and HFA in children [Abstract]. European Respiratory Journal. 2005; 26(Suppl 49) Abstract No. 1057.

Meijer 1995 \{published data only\} . Meijer GG, Postma DS, Mulder PG, van Aalderen WM. Long-term circadian effects of salmeterol in asthmatic children treated with inhaled corticosteroids. American Journal of Respiratory \& Critical Care Medicine. 1995; 152:1887-92. [PubMed: 8520751]

Morice 2008a $\quad$ \{published and unpublished data $\quad$. AstraZeneca. [accessed 4th January 2008] A 12week randomised, double-blind, parallel-group, multicentre phase-III study to compare the efficacy and safety of Symbicort ${ }^{\circledR}$ pMDI (budesonide/formoterol 80/4.5 mcg 2 actuations b.i.d., delivered dose) with that of Pulmicort ${ }^{\circledR}$ pMDI (budesonide $100 \mathrm{mcg} 2$ actuations b.i.d., metered dose) and Symbicort Turbuhaler®(budesonide/formoterol 80/4.5 mcg 2 actuations b.i.d., delivered dose) in children with asthma. 2007. http://www.astrazenecaclinicaltrials.com 
*Morice AH, Peterson S, Beckman O, Kukova Z. Efficacy and safety of a new pressurised metereddose inhaler formulation of budesonide/formoterol in children with asthma: A superiority and therapeutic equivalence study. Pulmonology Pharmacology Therapeutics. 2008; 21(1):152-9.

Morice 2008b \{published data only\} . AstraZeneca. [accessed 4th January 2008] A 12-week randomised, double-blind, parallel-group, multicentre phase-III study to compare the efficacy and safety of Symbicort@pMDI (budesonide/formoterol 80/4.5 mcg 2 actuations b.i.d., delivered dose) with that of Pulmicort ${ }^{\circledR p M D I}$ (budesonide $100 \mathrm{mcg} 2$ actuations b.i.d., metered dose) and Symbicort Turbuhaler ${ }^{(}$(budesonide/formoterol 80/4.5 mcg 2 actuations b.i.d., delivered dose) in children with asthma. 2007. http://www.astrazenecaclinicaltrials.com

*Morice AH, Peterson S, Beckman O, Kukova Z. Efficacy and safety of a new pressurised metereddose inhaler formulation of budesonide/formoterol in children with asthma: A superiority and therapeutic equivalence study. Pulmonology Pharmacology Therapeutics. 2008; 21(1):152-9.

Ortega-Cisneros 1998 \{published data only\} . Ortega-Cisnero M, Maldonado-Alaniz ML, Rosas Vargas MA, Sierra-Monge JJL. Salmeterol and inhaled beclomethasone versus high dose inhaled beclomethasone in the control of pediatric patients with moderate asthma. Annals of Allergy, Asthma and Immunology. 1998; 80:131.

Pohunek 2006a \{published and unpublished data\} . Pohunek P, Kuna P, De Boeck K. Budesonide/ formoterol improves lung function compared with budesonide alone in children with asthma [Abstract]. European Respiratory Journal. 2004; 24(Suppl 48):379s.

*Pohunek P, Kuna P, Jorup C, Boeck KD. Budesonide/formoterol improves lung function compared with budesonide alone in children with asthma. Pediatric Allergy and Immunology. 2006; 17(6): 458-65. [PubMed: 16925692]

Pohunek P, Matulka M, Rybnicek O, Kopriva F, Honomichlova H, Svobodova T. Dose-related efficacy and safety of formoterol (Oxis) Turbuhaler compared with salmeterol Diskhaler in children with asthma. Pediatric Allergy \& Immunology. 2004; 15(1):32-9. [PubMed: 14998380]

Pohunek 2006b \{published and unpublished data\} . Pohunek P, Kuna P, De Boeck K. Budesonide/ formoterol improves lung function compared with budesonide alone in children with asthma [Abstract]. European Respiratory Journal. 2004; 24(Suppl 48):379s.

*Pohunek P, Kuna P, Jorup C, Boeck KD. Budesonide/formoterol improves lung function compared with budesonide alone in children with asthma. Pediatric Allergy and Immunology. 2006; 17(6): 458-65. [PubMed: 16925692]

Pohunek P, Matulka M, Rybnicek O, Kopriva F, Honomichlova H, Svobodova T. Dose-related efficacy and safety of formoterol (Oxis) Turbuhaler compared with salmeterol Diskhaler in children with asthma. Pediatric Allergy \& Immunology. 2004; 15(1):32-9. [PubMed: 14998380]

Russell 1995 \{published data only\} . Glaxo Smith Kline (SALMP/AH91/D89). [accessed 9th June 2008] A phase III, multi-centre, double-blind, placebo controlled, parallel group study assessing the efficacy and safety of inhaled salmeterol xinafoate (Serevent ${ }^{\mathrm{TM}}$ ) 50 micrograms BD via the Diskhaler ${ }^{\mathrm{TM}}$ when added to the existing treatment of moderate to severe asthmatic children. 2006. www.ctr.gsk.co.uk

*Russell G, Williams DAJ, Weller P, Price JF. Salmeterol xinafoate on children on high dose inhaled steroids. Annals of Allergy, Asthma and Immunology. 1995; 75:423-28.

SAM104926 \{unpublished data only\} . Glaxo Smith Kline (SAM104926). [accessed 14/01/2008] A multicentre, randomised, double-blind, double dummy, parallel group study to compare the salmeterol/fluticasone propionate combination (SERETIDETM) at a dose of 50/100mcg twice daily and fluticasone propionate (FLIXOTIDETM) at a dose of $200 \mathrm{mcg}$ twice daily, both delivered via a dry powder inhaler (DISKUS ${ }^{\mathrm{TM}}$ ) for 12 weeks in asthma in children aged 4-11 years not controlled by inhaled corticosteroids alone at medium dose. 2007. http://ctr.gsk.co.uk

SAM40012a \{unpublished data only\} . Dorinsky P, Emmett A, Sutton L. Reduced risk for asthma exacerbations in pediatric patients receiving salmeterol plus inhaled corticosteroids (ICS) versus ICS alone [Abstract]. European Respiratory Journal. 2004; 24(Suppl 48):308s.

*Glasxo Smith Kline (SAM40012). A multicentre, randomised, double-blind, double-dummy, parallel group comparison of three treatments: 1) salmeterol/fluticasone propionate (SFC) $(50 / 100 \mathrm{mcg}$ strength) bd via DISKUS/ACCUHALER inhaler, 2) fluticasone propionate $200 \mathrm{mcg}$ bd via DISKUS/ACCUHALER inhaler, 3) fluticasone propionate 100mcg bd via DISKUS/ ACCUHALER inhaler in children aged 4-11 years with asthma. 2005 http://www.ctr.gsk.co.uk. 
SAM40012b \{unpublished data only $\quad$. Dorinsky P, Emmett A, Sutton L. Reduced risk for asthma exacerbations in pediatric patients receiving salmeterol plus inhaled corticosteroids (ICS) vs ICS alone [Abstract]. European Respiratory Journal. 2004; 24(Suppl 48):308s.

*Glaxo Smith Kline (SAM40012). A multicentre, randomised, double-blind, double-dummy, parallel group comparison of three treatments: 1) salmeterol/fluticasone propionate (SFC) $(50 / 100 \mathrm{mcg}$ strength) bd via DISKUS/ACCUHALER inhaler, 2) fluticasone propionate $200 \mathrm{mcg}$ bd via DISKUS/ACCUHALER inhaler, 3) fluticasone propionate 100mcg bd via DISKUS/ ACCUHALER inhaler in children aged 4-11 years with asthma. 2005. http://www.ctr.gsk.co.uk

SAM40100 \{unpublished data only\} . Glaxo Smith Kline (SAM40100). [accessed 4th January 2008] Randomised, double-blind, comparator study to demonstrate the superiority of salmeterol/ fluticasone propionate combination DISKUS ${ }^{\mathrm{TM}} 50 / 100 \mathrm{mcg}$ bd over fluticasone propionate DISKUS ${ }^{\mathrm{TM}} / \mathrm{ACCUHALER}^{\mathrm{TM}} 200 \mathrm{mcg}$ bd with respect to airway physiology in asthmatic children treated for 6 weeks. 2006. http://ctr.gsk.co.uk

SD 0390714 \{published data only\}. AstraZeneca Pharmaceuticals (SD 039 0714). [Accessed 21-02/2006] Efficacy and safety of budesonide/formoterol Turbuhaler® (160/4.5 mcg b.i.d. delivered dose) compared to budesonide Turbuhaler® (200 mcg b.i.d. metered dose) in steroidusing asthmatic adolescent patients. A double-blind, double-dummy, randomised, parallel group, phase III, multicentre study. 2005. http://www.astrazenecaclinicaltrials.com

SD 0390718 \{unpublished data only\}. AstraZeneca Pharmaceuticals (SD 039 0718). [accessed 4th January 2008] A twelve-week, randomized, double-blind, double-dummy trial of Symbicort ${ }^{\circledR}(40 / 4.5 \mathrm{mcg})$ versus its mono-products (budesonide and formoterol) in asthmatic children aged six to fifteen years. 2005. http://www.astrazenecaclinicaltrials.com

SD 0390719 \{unpublished data only\}. AstraZeneca Pharmaceuticals (SD 039 0719). [accessed 4th January 2008] A six-month, randomized, open-label safety study of Symbicort@ (160/4.5 mcg) compared to Pulmicort Turbuhaler® in asthmatic children aged 6 to 11 years. 2005. http:// www.astrazenecaclinicaltrials.com

SD 039 0725a \{published data only\} . AstraZeneca Pharmaceuticals (SD 039 0725). [accessed 4th January 2008] A twelve-week, randomized, double-blind, double-dummy, active-controlled study of Symbicort ${ }^{\circledR}$ pMDI administered once daily in children and adolescents 6 to 15 years of age with asthma. 2005. http://www.astrazenecaclinicaltrials.com

SD 039 0725b \{unpublished data only\} . AstraZeneca Pharmaceuticals (SD 039 0725). [accessed 4th January 2008] A twelve-week, randomized, double-blind, double-dummy, active-controlled study of Symbicort ${ }^{\circledR}$ pMDI administered once daily in children and adolescents 6 to 15 years of age with asthma. 2005. http://www.astrazenecaclinicaltrials.com

SFA100314 \{unpublished data only\} . Glaxo Smith Kline (SFA100314). [accessed 16th May 2008] A stratified, multicenter, randomized, double-blind, parallel group, 4-week comparison of fluticasone propionate/salmeterol DISKUS combination product 100/50mcg BID versus fluticasone propionate DISKUS 100mcg BID in pediatric and in adolescent subjects with activity-induced bronchospasm. 2007. http://www.ctr.gsk.co.uk

SFA100316 \{unpublished data only\} . Glaxo Smith Kline (SFA100316). [accessed 30th April 2008] A stratified, multicenter, randomized, double-blind, parallel group, 4-week comparison of fluticasone propionate/salmeterol DISKUS combination product 100/50mcg BID versus fluticasone propionate DISKUS 100mcg BID in pediatric and in adolescent subjects with activity-induced bronchospasm. 2006. http://ctr.gsk.co.uk

Simons 1997 \{published data only\} . Simons FE, Gerstner TV, Cheang MS. Tolerance to the bronchoprotective effect of salmeterol in adolescents with exercise-induced asthma using concurrent inhaled glucocorticoid treatment. Pediatrics. 1997; 99(5):655-9. [PubMed: 9113940]

Stelmach 2007 \{published data only\} . *Stelmach I, Grzelewskia T, Bobrowska-Korzeniowska M, Stelmach P, Kuna P. A randomized, double-blind trial of the effect of anti-asthma treatment on lung function in children with asthma. Pulmonology Pharmacology \& Therapeutics. 2007; 20:691-700.

Tal 2002 \{published data only\} . AstraZeneca Pharmaceuticals (SD 039 0353). [accessed 25th April 2008] Efficacy and safety of budesonide/formoterol Turbuhaler® in a fixed combination in steroid-using asthmatic children. http://www.astrazenecaclinicaltrials.com 
Tal, A.; Simon, G.; Vermeulen, JH. Symbicort ${ }^{\circledR}$ (Budesonide and formoterol in a single inhaler) is effective and well tolerated in children with asthma; Annual Thoracic Society 97th International Conference; San Francisco CA. May 18-23. 2001;

Tal, A.; Simon, G.; Vermeulen, JH.; Petru, V.; Cobos, N.; Everard, ML.; de Boeck, C.; Jerre, F. Symbicort (budesonide and formoterol in a single inhaler) improves lung function in children with asthma; International Paediatric Respiratory and Allergy Congress; Prague. April 1-4; 2001. p. 85

Tal, A.; Simon, G.; Vermeulen, JH.; Petru, V.; Cobos, N.; Everard, ML.; de Boeck, C.; Jerre, F. Symbicort (budesonide and formoterol in a single inhaler) is more effective that budesonide alone in children with asthma; International Paediatric Respiratory and Allergy Congress; Prague. April 1-4; 2001. p. 84-5.

Tal A, Simon G, Vermeulen JH, Petru V, Cobos N, Everard ML, De Boeck C, Naya I. Rapid and sustained improvements in lung function and symptom control with budesonide/ formoterol in adolescent asthma. European Respiratory Journal. 2001; 18(Suppl 33):494s.

*Tal A, Simon G, Vermeulen JH, Petru V, Cobos N, Everard ML, de Boeck K. Budesonide/ Formoterol in a single Inhaler versus inhaled corticosteroids alone in the treatment of asthma. Pediatric Pulmonology. 2002; 34(5):342-50. [PubMed: 12357478]

Vermeulen, JH.; Simon, G.; Tal, A. Symbicort ${ }^{\circledR}$ (Budesonide and formoterol in a single inhaler) improves lung function in asthmatic children aged 4-17 years; Annual Thoracic Society 97th International Conference; San Francisco CA. May 18-23. 2001;

Teper 2005 \{published data only\} . Teper, AM.; Zaragoza, SM.; Lubovich, S.; Rodriguez, VA.; Venalago, C.; Kofman, CD.; Vidauretta, SM. Effect of fluticasone propionate (FP) with or without salmeterol (S) on bronchial reactivity (BR) in children with mild to moderate persistent asthma [Abstract]; American Thoracic Society 2005 International Conference; San Diego, California. May 20-25; 2005. p. C47Poster: A5

Verberne 1998a \{published data only . Verberne AAPH, Frost C, Duiverman EJ, Grol MH, Kerribijn KF. Addition of salmeterol versus doubling the dose of beclomethasone in children with asthma. American Journal of Respiratory \& Critical Care Medicine. 1998; 158(1):213-9. [PubMed: 9655732]

Verberne 1998b \{published data only . Verberne AAPH, Frost C, Duiverman EJ, Grol MH, Kerribijn KF. Addition of salmeterol versus doubling the dose of beclomethasone in children with asthma. American Journal of Respiratory \& Critical Care Medicine. 1998; 158:213-19. [PubMed: 9655732]

Zimmerman 2004a \{published data only\} . *Zimmerman B, D’Urzo A, Berube D. Efficacy and safety of formoterol turbuhaler(R) when added to inhaled corticosteroid treatment in children with asthma. Pediatric Pulmonology. 2004; 37(2):122-7. [PubMed: 14730657]

Zimmerman B, D'Urzo A, Berube D. Efficacy and tolerability of formoterol turbuhaler(r) compared with placebo, in children (6-11 yr) with asthma poorly controlled with inhaled corticosteroids [abstract]. American Journal of Respiratory and Critical Care Medicine. 2002; 165(8 Suppl):A746.

Zimmerman B, D'Urzo A, Berube D. Efficacy and tolerability of formoterol Turbuhaler in 6-11 year old children with asthma, not adequately controlled with inhaled corticosteroids. European Respiratory Society Annual Congress. 2002:P2734.

Zimmerman 2004b \{published data only\} . *Zimmerman B, D'Urzo A, Berube D. Efficacy and safety of formoterol turbuhaler(R) when added to inhaled corticosteroid treatment in children with asthma. Pediatric Pulmonology. 2004; 37(2):122-7. [PubMed: 14730657]

Zimmerman B, D'Urzo A, Berube D. Efficacy and tolerability of formoterol turbuhaler(r) compared with placebo, in children (6-11 yr) with asthma poorly controlled with inhaled corticosteroids [abstract]. American Journal of Respiratory and Critical Care Medicine. 2002; 165(8 Suppl):A746.

Zimmerman B, D’Urzo A, Berube D. Efficacy and tolerability of formoterol Turbuhaler in 6-11 year old children with asthma, not adequately controlled with inhaled corticosteroids. European Respiratory Society Annual Congress. 2002:P2734. 


\section{References to studies excluded from this review}

Aldington 2006 \{published data only\} . Aldington S, Williams M, Weatherall M, Beasley R. Duration of action of the salmeterol/fluticasone combination inhaler administered in the evening: A randomized controlled trial in childhood asthma. Respirology. 2006; 11(5):638-42. [PubMed: 16916339]

Aubier 1999 \{published data only\} . Aubier M, Pieters WR, Schlosser NJ, Steinmetz KO. Salmeterol/fluticasone propionate $(50 / 500 \mathrm{mug})$ in combination in a Diskus(TM) inhaler (Seretide(TM)) is effective and safe in the treatment of steroid-dependent asthma. Respiratory Medicine. 1999; 93(12):876-84. [PubMed: 10653049]

Bergmann 2004 \{published data only\} . Bergmann KC, Lindemann L, Braun R, Steinkamp G. Salmeterol/fluticasone propionate (50/250 microg) combination is superior to double dose fluticasone (500 microg) for the treatment of symptomatic moderate asthma. Swiss Medical Weekly. 2004; 134(3-4):50-8. [PubMed: 14745658]

Borker 2005 \{published data only\} . Borker R, Emmett A, Jhingran P, Rickard K, Dorinsky P. Determining economic feasibility of fluticasone propionate-salmeterol vs montelukast in the treatment of persistent asthma using a net benefit approach and cost-effectiveness acceptability curves. Annals of Allergy, Asthma, \& Immunology. 2005; 95(2):181-9.

Boulet 2003 \{published data only\} . Boulet LP, Roberts J, Watson EG. Efficacy of salmeterol/ fluticasone propionate MDI versus higher dose fluticasone propionate in adolescent and adult asthmatics uncontrolled on fluticasone propionate alone [Abstract]. European Respiratory Journal. 2003; 22(Suppl 45) Abstract No: [P2799].

Bousquet 2005 \{published data only\}. Bousquet J, Gaugris S, Kocevar VS, Zhang Q, Yin DD, Polos PG, Bjermer L. Increased risk of asthma attacks and emergency visits among asthma patients with allergic rhinitis: A subgroup analysis of the improving asthma control trial. Clinical \& Experimental Allergy. 2005; 35(6):723-7. [PubMed: 15969661]

Bracamonte 2005 \{published data only\} . Bracamonte T, Schauer U, Emeryk A, Godwood A, Balsara S. Efficacy and safety of salmeterol/fluticasone propionate combination delivered by the Diskus or pressurised metered-dose inhaler in children with asthma. Clinical Drug Investigation. 2005; 25(1):1-11. [PubMed: 17523742]

Bruce 2005 \{published data only\} . Bruce SA, Scherer YK. Maintenance and symptom relief with budesonide plus formoterol reduced severe asthma exacerbations. Evidence-Based Nursing. Jul. 2005 8(3):78. [PubMed: 16021707]

Bruggenjurgen 2005 \{published data only\} . Bruggenjurgen B, Selim D, Kardos P, Richter K, Vogelmeier C, Roll S, et al. Economic assessment of adjustable maintenance treatment with budesonide/formoterol in a single inhaler versus fixed treatment in asthma. Pharmacoeconomics. 2005; 23(7):723-31. [PubMed: 15987228]

Buchvald 2002 \{published data only\} . Buchvald FF, Bisgaard H. Comparison of add-on of leukotriene receptor antagonist vs. long-acting beta2-agonist of FeNO in asthmatic children on regular inhaled budesonide. European Respiratory Journal. 2002; 20(Suppl 38):431s.

Buhl 2004 \{published data only\} . Buhl R, Kardos P, Richter K, Meyer-Sabellek W, Bruggenjurgen B, Willich SN, Vogelmeier C. The effect of adjustable dosing with budesonide/ formoterol on health-related quality of life and asthma control compared with fixed dosing. Current Medical Research \& Opinion. 2004; 20(8):1209-20. [PubMed: 15324523]

Caffey 2005 \{published data only\} . Caffey LF, Raissy HH, Marshik P, Kelly HW. A crossover comparison of fluticasone propionate and montelukast on inflammatory indices in children with asthma. Pediatric Asthma Allergy \& Immunology. 2005; 18(3):123-30.

Chopra 2005 \{published data only\} . Chopra N, Williams M, Rimmer M, Kahl L, Jenkins M. SMO30006 and SMO30007 International Study Teams. Salmeterol HFA is as effective as salmeterol CFC in children and adults with persistent asthma. Respiratory Medicine. 2005; 99(SUPPL 1):S1-S10. [PubMed: 15777603]

Chuchalin 2005 \{published data only\} . Chuchalin A, Kasl M, Bengtsson T, Nihlen U, Rosenborg J. Formoterol used as needed in patients with intermittent or mild persistent asthma. Respiratory Medicine. 2005; 99(4):461-70. [PubMed: 15763453] 
Cowan 2004 \{published data only\} . Cowan S, Ernst P, Cartier A, Boulet LP. A population-based evaluation of a regional asthma education centre. Canadian Respiratory Journal. 2004; 11(1):39_ 44. [PubMed: 15010731]

Daviskas 2005 \{published data only\} . Daviskas E, Anderson SD, Shaw J, Eberl S, Seale JP, Yang IA, Young IH. Mucociliary clearance in patients with chronic asthma: effects of beta agonists. Respirology. 2005; 10(4):426-35. [PubMed: 16135164]

Delaronde 2005 \{published data only\} . Delaronde S, Peruccio DL, Bauer BJ. Improving asthma treatment in a managed care population. American Journal of Managed Care. 2005; 11(6):361-8. [PubMed: 15974555]

Dubus 2003 \{published data only\} . Dubus JC, Mely L, Huiart L, Marguet C, Le Roux P. Reseau de Recherche Clinique en Pneumologie Pediatrique. Cough after inhalation of corticosteroids delivered from spacer devices in children with asthma. Fundamental \& Clinical Pharmacology. 2003; 17(5):627-31. [PubMed: 14703724]

Emeryk 2003 \{published data only\} . Emeryk A, Bracamonte T, Schauer U, Balsara S, Athisegaran R. Efficacy of salmeterol/fluticasone propionate $(50 / 100 \mathrm{mcg} /$ day) delivered via the Diskus and MDi in children with asthma [Abstract]. European Respiratory Journal. 2003; 22(Suppl 45) Abstract No: [P1985].

Everden 2004 \{published data only\} . Everden P, Campbell M, Harnden C, McGoldrick H, Bodalia B, Manion V, Reynia S. Eformoterol Turbohaler compared with salmeterol by dry powder inhaler in asthmatic children not controlled on inhaled corticosteroids. Pediatric Allergy \& Immunology. 2004; 15(1):40-7. [PubMed: 14998381]

Fardon 2005 \{published data only\} . Fardon TC, Lee DK, Hodge MR, Lipworth BJ. Addition of fexofenadine to inhaled corticosteroid therapy to reduce inflammatory biomarkers in atopic asthma. Annals of Allergy, Asthma, \& Immunology. 2005; 95(3):259-65.

Grady 1995 \{published data only\} . Grady J, Dockhorn R, Blake K. Effects of inhaled salmeterol powder in adult and adolescent asthmatics using concurrent inhaled corticosteroids [abstract]. European Respiratory Journal. Supplement. 1995; 8(Suppl 19):2S.

Holgate 2004 \{published data only\} . Holgate ST, Chuchalin AG, Hebert J, Lotvall J, Persson GB, Chung KF, et al. Efficacy and safety of a recombinant anti-immunoglobulin E antibody (omalizumab) in severe allergic asthma. Clinical \& Experimental Allergy. 2004; 34(4):632-8. [PubMed: 15080818]

Holt 2005 \{published data only\} . Holt S, Ryder-Lewis S, Masoli M, Weatherall M, Beasley R. Fixed and adjustable dose asthma action plans based on combination therapy: a pilot study. Respirology. 2005; 10(4):497-503. [PubMed: 16135174]

Ilowite 2004 \{published data only\} . Ilowite J, Webb R, Friedman B, Kerwin E, Bird SR, Hustad $\mathrm{CM}$, Edelman JM. Addition of montelukast or salmeterol to fluticasone for protection against asthma attacks: a randomized, double-blind, multicenter study. Annals of Allergy, Asthma, \& Immunology. 2004; 92(6):641-8.

Jenkins 2005 ppublished data only\} . Jenkins CR, Thien FCK, Wheatley JR, Reddel HK. Traditional and patient-centred outcomes with three classes of asthma medication. European Respiratory Journal. 2005; 26(1):36-44. [PubMed: 15994387]

Karaman 2007 \{published data only\} . Karaman O, Arli O, Uzuner N, Islekel H, Babayigit A, Olmez D, et al. The effectiveness of asthma therapy alternatives and evaluating the effectivity of asthma therapy by interleukin-13 and interferon gamma levels in children. Allergy \& Asthma Proceedings. 2007; 28(2):204-9. [PubMed: 17479606]

Lara-Perez 2005 \{published data only\} . Lara-Perez, EA. Emprovement of the asthma in children using salmeterol and fluticasone (Seretide) in inhaled combination [Abstract]; XIX World Allergy Organization Congress; 2005. Abstract 861

Levy 2005 \{published data only\} . Levy R, Pinnas J, Milgrom H, Smith J, Yegen U. Safety and efficacy in children with persistent asthma treated with formoterol 10 mug BID delivered via Certihaler: A novel multidose dry-powder inhaler. Pediatric Asthma Allergy \& Immunology. 2005; 18(1):25-35.

LOCCS \{published data only\}. American Lung Association Asthma Clinical Research. Randomized comparison of strategies for reducing treatment in mild persistent asthma. New England Journal of Medicine. 2007; 356(20):2027-39. [PubMed: 17507702] 
Matthys 2004 \{published data only\} . Matthys H, Behling B, Behling E. Comparison of formoterol (12 and 6 mug b.i.d) versus terbutaline $(0.5 \mathrm{mg}$ b.i.d) with equal doses of inhaled budesonide $(0.2$ $\mathrm{mg}$ bid) in 246 children with mild to moderate asthma. Atemwegs-Und Lungenkrankheiten. 2004; 30(5):251-6.

Miraglia 2007 \{published data only\} . Miraglia del Giudice, M.; Capristo, M.; Amelio, R.; Rocco, A.; Fusco, N.; Brunese, FP. Combined fluticasone propionate/salmeterol (diskus) vs fluticasone propionate (Diskus) in the control of persistent asthma in children; American Thoracic Society 99th International Conference; 2003; :A117 Poster D78

*Miraglia del Giudice M, Piacentini GL, Capasso M, Capristo C, Maiello N, Boner AL, Capristo AF. Formoterol, montelukast, and budesonide in asthmatic children: Effect on lung function and exhaled nitric oxide. Respiratory Medicine. 2007; 101(8):1809-13. [PubMed: 17418554]

Mitchell 2005 \{published data only\} . Mitchell EA, Didsbury PB, Kruithof N, Robinson E, Milmine M, Barry M, Newman J. A randomized controlled trial of an asthma clinical pathway for children in general practice. Acta Paediatrica. 2005; 94(2):226-33. [PubMed: 15981759]

Mitra 2003 \{published data only\} . Mitra A, Sims EJ, Mukhopadhyay S, Lipworth BJ. Low-dose inhaled corticosteroid (ICS) confers optimal anti-inflammatory effects while additional bronchodilator activity is afforded by salmeterol in mild persistent asthmatic children [Abstract]. Journal of Allergy and Clinical Immunology. 2003; 111(2 Suppl):S145.

Morice 2005 \{published data only . Morice AH, Hochmuth L, Puterman A, Artheden L, Beckman O. Comparable safety of a novel budesonide/formoterol pMDI versus budesonide/formoterol Turbuhaler in adolescents and adults with asthma [Abstract]. Journal of Allergy \& Clinical Immunology. 2005; 115(2 Suppl):S3.

Morice 2005a \{published data only\}. Morice AH, Osmanliev D, Arheden L, Beckman O. Therapeutic equivalence of a novel budesonide/formoterol pMDI versus budesonide/formoterol Turbuhaler in adolescents and adults with asthma [Abstract]. Journal of Allergy \& Clinical Immunology. 2005; 115(2 Suppl):S2.

Murray 2004 \{published data only\} . Murray J, Rosenthal R, Somerville L, Blake K, House K, Baitinger L, VanderMeer A, Dorinsky P. Fluticasone propionate and salmeterol administered via Diskus compared with salmeterol or fluticasone propionate alone in patients suboptimally controlled with short-acting beta2-agonists. Annals of Allergy, Asthma, \& Immunology. 2004; 93(4):351-9.

Nathan 2005 \{published data only\} . Nathan RA, Yancey SW, Waitkus-Edwards K, Prillaman BA, Stauffer JL, Philpot E, et al. Fluticasone propionate nasal spray is superior to montelukast for allergic rhinitis while neither affects overall asthma control. Chest. 2005; 128(4):1910-20. [PubMed: 16236835]

Nelson 2006 \{published data only\} . Nelson HS, Weiss ST, Bleecker ER, Yancey SW, Dorinsky PM, SMART Study Group. The Salmeterol Multicenter Asthma Research Trial: a comparison of usual pharmacotherapy for asthma or usual pharmacotherapy plus salmeterol. Chest. 2006; 129(1):15-26. [PubMed: 16424409]

Nguyen 2005 \{published data only\} . Nguyen WT, Stewart C, Fisher K, Tolley E, Lew Db, Self Th. Maintenance Asthma Treatment With Fluticasone/Salmeterol Combination Via Diskus: Effect on Outcomes in Inner-City Children Enrolled in Tenncare. Allergy and Asthma Proceedings. 2005; 26(2):129-34. [PubMed: 15971472]

O’Byrne 2001 \{published data only\} . Barnes PJ, O'Byrne PM, Rodriguez-Roisin R, Runnerstrom E, Sandstrom T, Svensson K, Tattersfield A. Oxis and Pulmicort turbuhaler in the management of asthma OPTIMA international study group. Treatment of mild persistent asthma with low doses of inhaled Budesonide alone or in combination with Formoterol. Thorax. 2000; 55(Suppl 3):A4.

Barnes PJ, O’Byrne PM, Rodriguez-Roisin R, Runnerstrom E, Sandstrom T, Svensson K, Tattersfield A. Treatment of mild persistent asthma with low doses of inhaled budesonide alone or in combination with formoterol. For the Oxis and Pulmicort Turbuhaler in the Management of Asthma (OPTIMA) international study group. Thorax. 2000; 55(Suppl 3):s5.

Bisgaard H, Hultquist C. Budesonide/formoterol for maintenance and as needed - a new approach to asthma management in children [Abstract]. European Respiratory Journal. 2005; 26(Suppl 49) Abstract No. 1060. 
Jönsson BG, Berggren FE, Svensson K, O’Byrne PM. Budesonide and formoterol in mild persistent asthma compared with doubling the dose of budesonide - a cost-effectiveness analysis. European Respiratory Journal. 2001; 18(Suppl 33):517s.

Jönsson BG, Berggren FE, Svensson K, O’Byrne PM. Economic results of adding formoterol to budesonide in mild persistent asthma. European Respiratory Journal. 2001; 18(Suppl 33):331s.

*O'Byrne PM, Barnes PJ, Rodriguez-Roisin R, Runnerstrom E, Sandstrom T, Svensson K, Tattersfield A. Low dose inhaled budesonide and formoterol in mild persistent asthma: The OPTIMA randomized trial. American Journal of Respiratory \& Critical Care Medicine. 2001; 164(8 pt 1): 1392-7. [PubMed: 11704584]

O’Byrne PM, Barnes PJ, Rodriguez-Roisin R, Sandtröm T, Tattersfield AE, Runnerström EM, Svensson K. Addition of formoterol Turbuhaler ${ }^{\circledR}$ to budesonide Tubuhaler ${ }^{\circledR}$ is safe and well tolerated in the long-term treatment of mild asthma: results from the OPTIMA trial. European Respiratory Journal. 2001; 18(Suppl 33):330s.

Pearlman 2004 \{published data only\} . Pearlman DS, Peden D, Condemi JJ, Weinstein S, White M, Baitinger L, Scott C, Ho S-Y, House K, Dorinsky P. Efficacy and safety of fluticasone propionate/salmeterol HFA 134A MDI in patients with mild-to-moderate persistent asthma. Journal of Asthma. 2004; 41(8):797-806. [PubMed: 15641629]

Peroni 2005 \{published data only\} . Peroni DG, Piacentini GL, Bodini A, Ress M, Costella S, Boner AL. Montelukast versus formoterol as second-line therapy in asthmatic children exposed to relevant allergens. Allergy \& Asthma Proceedings. 2005; 26(4):283-6. [PubMed: 16270721]

Pijnenburg 2005 \{published data only\} . Pijnenburg MW, Bakker EM, Hop WC, De Jongste JC. Titrating steroids on exhaled nitric oxide in children with asthma: A randomized controlled trial. American Journal of Respiratory \& Critical Care Medicine. 2005; 172(7):831-6. [PubMed: 15976380]

Prieto 2005 \{published data only . Prieto L, Gutierrez V, Perez-Frances C, Badiola C, Lanuza A, Bruno L, Ferrer A. Effect of fluticasone propionate-salmeterol therapy on seasonal changes in airway responsiveness and exhaled nitric oxide levels in patients with pollen-induced asthma. Annals of Allergy, Asthma, \& Immunology. 2005; 95(5):452-61.

Renzi 2005 \{published data only\} . Renzi, PM.; Frassen, E.; Stat, P.; Watson, EG. Salmeterol/ fluticasone propionate diskus (ADVAIR) 50/100mcg bid improves asthma outcomes compared with fluticasone propionate (FLOVENT) diskus $100 \mathrm{mcg}$ bid when used as initial maintenance treatment in adult and adolescent subjects with symptomatic persistent asthma [Abstract]; American Thoracic Society 2005 International Conference; San Diego, California. May 20-25; 2005. p. C33Poster: F65

SAM30002 \{unpublished data only\} . SAM30002 (GSK). A randomised, double blind, doubledummy, parallel-group, twelve week comparison of salmeterol/fluticasone propionate (FP) DISKUS/ACCUHALER 50/100mcg bd. with budesonide $200 \mathrm{mcg}$ bd. plus formoterol $4.5 \mathrm{mcg}$ bd. (both by breath-activated dry powder inhaler [BADPI]) in adult and adolescent asthmatics. 2004 Www.Clinicalstudyresults.Org.

SAM40101 \{unpublished data only\} . Glaxo Smith Kline. [accessed 4th January 2008] A pilot single centre, randomised, double-blind, crossover study to demonstrate the superiority of Salmeterol/fluticasone propionate combination product 50/100mcg bd versus fluticasone propionate $100 \mathrm{mcg}$ bd when treated for two weeks with respect to activity levels in children aged 7-15 years. 2006. http://ctr.gsk.co.uk

SAS30021 \{unpublished data only\} . *SAS30021. [Accessed 28th April 2008] A Stratified, randomized, double-blind, placebo-controlled, parallel-group, 12-week trial evaluating the safety and efficacy of the fluticasone propionate/salmeterol DISKUS combination product $100 / 50 \mathrm{mcg}$ once daily versus fluticasone propionate DISKUS $100 \mathrm{mcg}$ once daily and placebo in symptomatic pediatric subjects (4-11 years) with asthma. 2005. http://www.ctr.gsk.co.uk

Schauer 2003 \{published data only\} . Schauer U, Bracamonte T, Emeryk A, Balsara S, Athisegaran R. Clinincal equivalence of salmeterol/fluticasone propionate combination 50/100mcg BID delivered via Diskus or CFC - free metered dose inhaler (MDI) in children with asthma [Abstract]. European Respiratory Journal. 2003; 22(Suppl 45) Abstract No: [P1986].

Scicchitano 2004a \{published data only\} . Scicchitano R, Aalbers R, Ukena D, Manjra A, Fouquert L, Centann S, et al. Efficacy and safety of budesonide/formoterol single inhaler therapy versus a 
higher dose of budesonide in moderate to severe asthma. Current Medical Research \& Opinion. 2004; 20(9):1403-18. [PubMed: 15383189]

Selroos 2004 \{published data only\} . Selroos O, Lofroos AB, Pietinalho A, Riska H. Asthma control and steroid doses 5 years after early or delayed introduction of inhaled corticosteroids in asthma: A real-life study. Respiratory Medicine. 2004; 98(3):254-62. [PubMed: 15002762]

SFCF3001 \{unpublished data only\} . GlaxoSmithKline. A multicentre, randomised, double-blind, double-dummy, parallel-group study to establish equivalence of the salmeterol/fluticasone propionate combination product $(50 / 250 \mu \mathrm{g})$ via either the reservoir powder inhalation device (RPID) or via the Diskus Inhaler over 12 weeks in adolescents and adults with moderate asthma. 2004. Www.Clinicalstudyresults.Org

SFCF3002 \{unpublished data only\} . SFCF3002 (GSK). A multicentre, randomised, double-blind, double-dummy, parallel-group study to establish equivalence of the fluticasone propionate/ salmeterol combination (FSC) product $(100 / 50 \mu \mathrm{g})$ via either the reservoir powder inhalation device (RPID) or via the Diskus inhaler over 12 weeks in children with asthma. 2004. www.clinicalstudyresults.org

Sienra-Monge 2004 \{published data only\} . Sienra-Monge JJL, Del Rio-Navarro BE, AlvarezAmador M, Blandon-Vijil V, Gomez Chico-Velasco R. The Quality of Life of the Asthmatic Child Caregiver. Gaceta Medica De Mexico. 2004; 140(2):139-45. [PubMed: 15162946]

Sorkness 2007 \{published and unpublished data\} . *Sorkness CA, Lemanske RF Jr, Mauger DT, Boehmer SJ, Chinchilli VM, Martinez FD, et al. Long-term comparison of 3 controller regimens for mild-moderate persistent childhood asthma: The Pediatric Asthma Controller Trial. Journal of Allergy \& Clinical Immunology. 2007; 119(1):64-72. [PubMed: 17140647]

Stelmach 2008 \{published data only\} . Stelmach I, Grzelewski T, Jerzynska J, Kuna P. A randomized, double-blind trial on the effect of treatment with montelukast, budesonide, montelukast with budesonide, formoterol with budesonide on lung function and clinical symptoms in children with asthma [Abstract]. Journal of Allergy \& Clinical Immunology. 2005; 115(2 Suppl):S151.

*Stelmach I, Grzelewski T, Majak P, Jerzynska J, Stelmach W, Kuna P. Effect of different antiasthmatic treatments on exercise-induced bronchoconstriction in children with asthma. Journal of Allergy and Clinical Immunology. 2007 Vol. Epub ahead of print.

Storms 2004 \{published data only\}. Storms W, Chervinsky P, Ghannam AF, Bird S, Hustad CM, Edelman JM, et al. A comparison of the effects of oral montelukast and inhaled salmeterol on response to rescue bronchodilation after challenge. Respiratory Medicine. 2004; 98(11):1051-62. [PubMed: 15526805]

van den Toorn 2005 \{published data only\} . van den Toorn LM, Prins JB, de Jongste JC, Leman K, Mulder PG, Hoogsteden HC, Overbeek SE. Benefit from anti-inflammatory treatment during clinical remission of atopic asthma. Respiratory Medicine. 2005; 99(6):779-87. [PubMed: 15878496]

Vogelmeier 2005 \{published data only\} . Vogelmeier C, D’Urzo A, Pauwels R, Merino JM, Jaspal M, Boutet S, et al. Budesonide/formoterol maintenance and reliever therapy: An effective asthma treatment option? European Respiratory Journal. 2005; 26(5):819-28. [PubMed: 16264042]

Von Berg 2003 \{published data only\} . Von Berg A, Papageorgiou Saxoni F, Wille S, Carrillo T, Kattamis C, Helms PJ. Efficacy and tolerability of formoterol turbuhaler in children. International Journal of Clinical Practice. 2003; 57(10):852-856. [PubMed: 14712884]

Weiler 2005 \{published data only\} . Weiler JM, Nathan RA, Rupp NT, Kalberg CJ, Emmett A, Dorinsky PM. Effect of fluticasone/salmeterol administered via a single device on exerciseinduced bronchospasm in patients with persistent asthma. Annals of Allergy, Asthma and Immunology. 2005; 94(1):65-72.

You-Ning 2005 \{published data only\} . You-Ning L, Humphries M, Du X, Wang L, Jiang J. Efficacy and safety of salmeterol/fluticasone propionate delivered via a hydrofluoroalkane metered dose inhaler in Chinese patients with moderate asthma poorly controlled with inhaled corticosteroids. International Journal of Clinical Practice. 2005; 59(7):754-9. [PubMed: 15963199] 


\section{Additional references}

Adams 2005 . Adams NP, Bestall JC, Malouf R, Lasserson TJ, Jones P. Beclomethasone versus placebo for chronic asthma. Cochrane Database of Systematic Reviews. 2005; (1) DOI: 10.1002/14651858.CD002738.Pub2.

Adams 2008a . Adams NP, Bestall JC, Lasserson TJ, Jones P, Cates CJ. Fluticasone versus placebo for chronic asthma in adults and children. Cochrane Database of Systematic Reviews. 2008; (4) DOI: 10.1002/14651858.CD003135.Pub4.

Adams 2008b . Adams NP, Bestall JC, Jones P, Lasserson TJ, Griffiths B, Cates CJ. Fluticasone at different doses for chronic asthma in adults and children. Cochrane Database of Systematic Reviews. 2008; (4) DOI: 10.1002/14651858.CD003534.pub3.

Bateman 2008 . Bateman E, Nelson H, Bousquet J, Kral K, Sutton L, Ortega H, et al. Meta-analysis: Effects of adding salmeterol to inhaled corticosteroids on serious asthma-related events. 2008; 149(1) www.annals.org. Epub.

Bisgaard 2003 . Bisgaard H. Effect of long-acting B2-agonists on exacerbation rates of asthma in children. Pediatric Pulmonology. 2003; 36:391-8. [PubMed: 14520721]

BTS 2007 . British Thoracic Society. British Guideline on the Management of Asthma. 2007 BTS website www.brit-thoracic.org.uk.

Canadian Paediatric Asthma Consensus Guidelines . Becker A, Bérubé D, Chad Z, Dolovich M, Ducharme F, D'Urzo T, et al. Canadian Pediatric Asthma Consensus Guidelines, 2003 (updated to December 2004). Canadian Medical Association Journal. 2005; 173(6 Suppl)

Cates 2008a . Cates CJ, Cates MJ. Regular treatment with salmeterol for chronic asthma: serious adverse events. Cochrane Database of Systematic Reviews. 2008; (3) DOI: 10.1002/14651858.CD006363.pub2.

Cates 2009a . Cates CJ, Lasserson TJ, Cates MJ. Regular treatment with formoterol and inhaled steroids for chronic asthma: serious adverse events. Cochrane Database of Systematic Reviews. 2009; (2) DOI: 10.1002/14651858.CD006924.pub2.

Cates 2009b . Cates CJ, Cates MJ, Lasserson TJ. Regular treatment with salmeterol and inhaled steroids for chronic asthma: serious adverse events. Cochrane Database of Systematic Reviews. 2009; (3) DOI: 10.1002/14651858.CD006922.pub2.

Cochrane Handbook . Higgins, JPT.; Green, S., editors. Cochrane Handbook for Systematic Reviews of Interventions Version 5.0.0 [updated February 2008]. The Cochrane Collaboration; 2008. Available from www.cochrane-handbook.org

DerSimonian 1986 . DerSimonian R, Laird N. Meta-analysis in clinical trials. Controlled Clinical Trials. 1986; 7:177-88. [PubMed: 3802833]

Ducharme 2006 . Ducharme F, Lasserson TJ, Cates CJ. Long-acting beta2-agonists versus antileukotrienes as add-on therapy to inhaled corticosteroids for chronic asthma. Cochrane Database of Systematic Reviews. 2006; (4) DOI: 10.1002/14651858.CD003137.pub3.

GINA 2007 . Global Initiative for Asthma. Global Strategy for Asthma Management and Prevention. 2007. NIH PublicationAvailable at: http://www.ginasthma.com

Higgins 2003 . Higgins JPT, Thompson SG, Deeks JJ, Altman DG. Measuring inconsistency in meta-analyses. BMJ. 2003; 327(557):560.

Manning 2008 . Manning P, Gibson PG, Lasserson TJ. Ciclesonide versus placebo for chronic asthma in adults and children. Cochrane Database of Systematic Reviews. 2008; (2) DOI: 10.1002/14651858.CD006217.Pub2.

NAC Guidelines 2006 . National Asthma Council. Asthma Management Handbook; Australia: 2006. www.nationalasthma.org.au

NIH Publication 2007 . National Asthma Education and Prevention Program. NIH Publication. National Heart, Lung and Blood Institute; Bethesda, MD: 2007. Expert Panel Report 3 (EPR 3) Guidelines for the Diagnosis and Management of Asthma. Available at: http:// www.nhlbi.nih.gov/guidelines/asthma/index.htm

Sharek 1999 . Sharek PJ, Bergman DA, Ducharme F. Beclomethasone for asthma in children: effects on linear growth. Cochrane Database of Systematic Reviews. 1999; (3) DOI: 10.1002/1465. 
Walters 2007 . Walters EH, Gibson PG, Lasserson TJ, Walters JAE. Long-acting beta2-agonists for chronic asthma in adults and children where background therapy contains varied or no inhaled corticosteroid. Cochrane Database of Systematic Reviews. 2007; (1) Art. No.: CD001385. DOI: 10.1002/14651858.CD001385.pub2.

\section{References to other published versions of this review}

Greenstone 2005 . Greenstone IR, Ni Chroinin MN, Masse V, Danish A, Magdalinos H, Zhang X, Ducharme FM. Combination of inhaled long-acting beta2-agonists and inhaled steroids versus higher dose of inhaled steroids in children and adults with persistent asthma. Cochrane Database of Systematic Reviews. 2005; (4) DOI: 10.1002/14651858.CD005533.

Ni Chroinin 2005 . Ni Chroinin M, Greenstone IR, Danish A, Magdolinos H, Masse V, Zhang X, Ducharme FM. Long-acting beta2-agonists versus placebo in addition to inhaled corticosteroids in children and adults with chronic asthma. Cochrane Database of Systematic Reviews. 2005; (4) DOI: 10.1002/14651858.CD005535.

*Indicates the major publication for the study 


\section{PLAIN LANGUAGE SUMMARY}

Addition of long-acting beta-agonists to inhaled corticosteroids for chronic asthma in children

Most consensus statements recommend the use of long-acting B2-agonists (LABA) as adjunct therapy to inhaled corticosteroids for poorly controlled asthma. The purpose of this review was to identify the benefits and safety profile of adding long-acting B2agonists to inhaled corticosteroids in asthmatic children. Based on the identified paediatric randomised trials, the addition of long-acting 32 -agonists did not significantly reduce the risk of asthma exacerbations requiring rescue systemic steroids, but improved lung function compared to ongoing treatment with a similar dose of inhaled corticosteroids. There was no evidence of increased serious side effects or withdrawals with the addition of long-acting 32 -agonists. Compared to doubling the dose of inhaled corticosteroids, the combination of LABA and inhaled steroids did not lead to a significant reduction in the rate of moderate exacerbations or hospital admissions, but it improved lung function and lead to greater growth. 


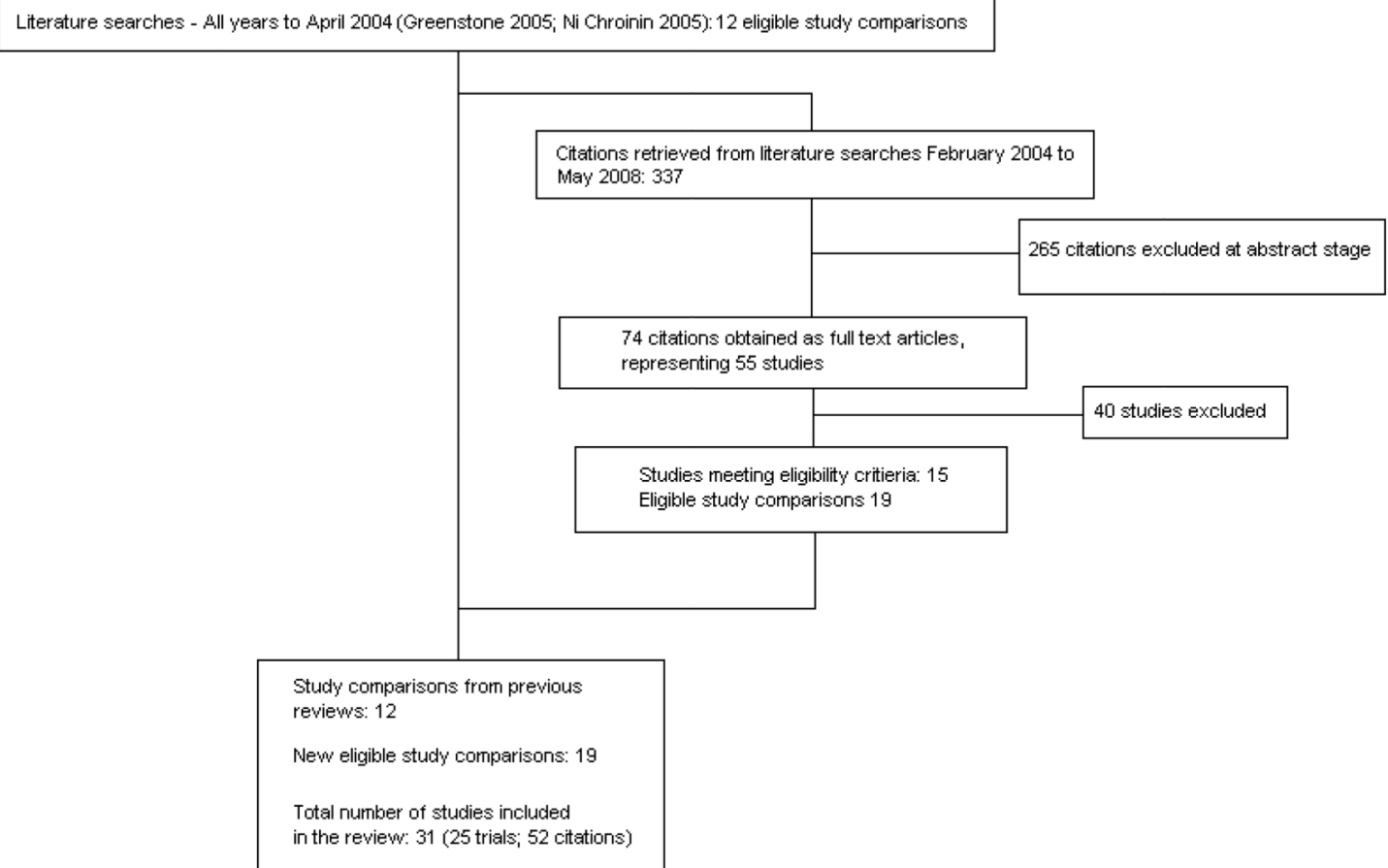

Figure 1.

Flow diagram of literature search results for review: Addition of long-acting beta-agonists to inhaled corticosteroids for chronic asthma in children 


\begin{tabular}{|c|c|c|c|c|c|c|}
\hline & 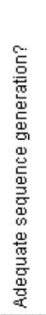 & 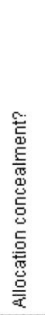 & $\begin{array}{l}\text { 히 } \\
\text { 言 } \\
\text { 言 }\end{array}$ & 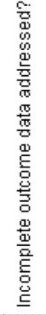 & 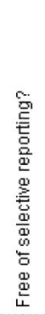 & 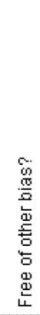 \\
\hline Akpinarli 1999 & $?$ & $?$ & $\odot$ & $?$ & $\odot$ & ? \\
\hline Bisgaard 2006 & $\odot$ & $?$ & $\odot$ & $?$ & $\rightarrow$ & $?$ \\
\hline Heuck 2000 & + & $?$ & $\odot$ & $?$ & (†) & $?$ \\
\hline Langton Hewer 1995 & $?$ & $?$ & + & $?$ & (†) & $?$ \\
\hline Malone 2005 & ๑ & $\odot$ & $\odot$ & $\theta$ & $\odot$ & + \\
\hline Meijer 1995 & $?$ & $?$ & $\odot$ & $?$ & $?$ & $?$ \\
\hline Morice 2008a & $\odot$ & $?$ & $\odot$ & $?$ & $\odot$ & $\odot$ \\
\hline Morice $2008 \mathrm{~b}$ & + & $?$ & 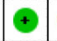 & $?$ & $\odot$ & $\odot$ \\
\hline Ortega-Cisneros 1998 & $?$ & $?$ & ○ & $?$ & $?$ & $?$ \\
\hline Pohunek 2006a & (†) & $\odot$ & + & $?$ & $?$ & + \\
\hline Pohunek 2006b & $\odot$ & $\odot$ & $\odot$ & $?$ & $?$ & + \\
\hline Russell 1995 & $\odot$ & $\odot$ & $\odot$ & - & $\odot$ & $?$ \\
\hline SAM104926 & $\odot$ & $\odot$ & $\odot$ & $?$ & $?$ & $?$ \\
\hline SAM40012a & + & $\odot$ & + & $?$ & $?$ & $?$ \\
\hline SAM40012b & + & $\odot$ & + & $?$ & $?$ & $?$ \\
\hline SAM 40100 & + & $\odot$ & $\odot$ & $?$ & $?$ & $?$ \\
\hline SD 0390714 & $?$ & $?$ & $\oplus$ & $?$ & $?$ & $\odot$ \\
\hline SD 0390718 & $?$ & $?$ & $\odot$ & $?$ & $?$ & $\odot$ \\
\hline SD 0390719 & $?$ & $?$ & ○ & $?$ & $\theta$ & + \\
\hline SD $0390725 \mathrm{a}$ & $?$ & $?$ & 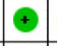 & $?$ & $\theta$ & + \\
\hline$S D 0390725 b$ & $?$ & $?$ & $\odot$ & $?$ & $\theta$ & + \\
\hline SFA100314 & + & $\odot$ & $\odot$ & $?$ & + & $?$ \\
\hline SFA100316 & $\odot$ & $\odot$ & $\odot$ & $?$ & 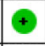 & $?$ \\
\hline Simons 1997 & $\odot$ & $?$ & $\odot$ & $\odot$ & $\odot$ & $?$ \\
\hline Stelmach 2007 & ๑ & $?$ & $\odot$ & + & $?$ & + \\
\hline Tal 2002 & + & ๑ & $\odot$ & $?$ & $?$ & $?$ \\
\hline Teper 2005 & $?$ & $?$ & $?$ & $?$ & $?$ & $?$ \\
\hline Verberne 1998a & $\odot$ & $\odot$ & $\bullet$ & $?$ & $\odot$ & $?$ \\
\hline Verberne $1998 \mathrm{~b}$ & $\bullet$ & $\odot$ & $\bullet$ & $?$ & $\odot$ & $?$ \\
\hline Zimmerman 2004a & $?$ & $?$ & $\odot$ & $?$ & $\odot$ & 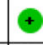 \\
\hline Zimmerman 2004b & $?$ & $?$ & $\odot$ & $?$ & 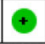 & $\oplus$ \\
\hline
\end{tabular}

Figure 2.

Methodological quality summary: review authors' judgments about each methodological quality item for each included study. 


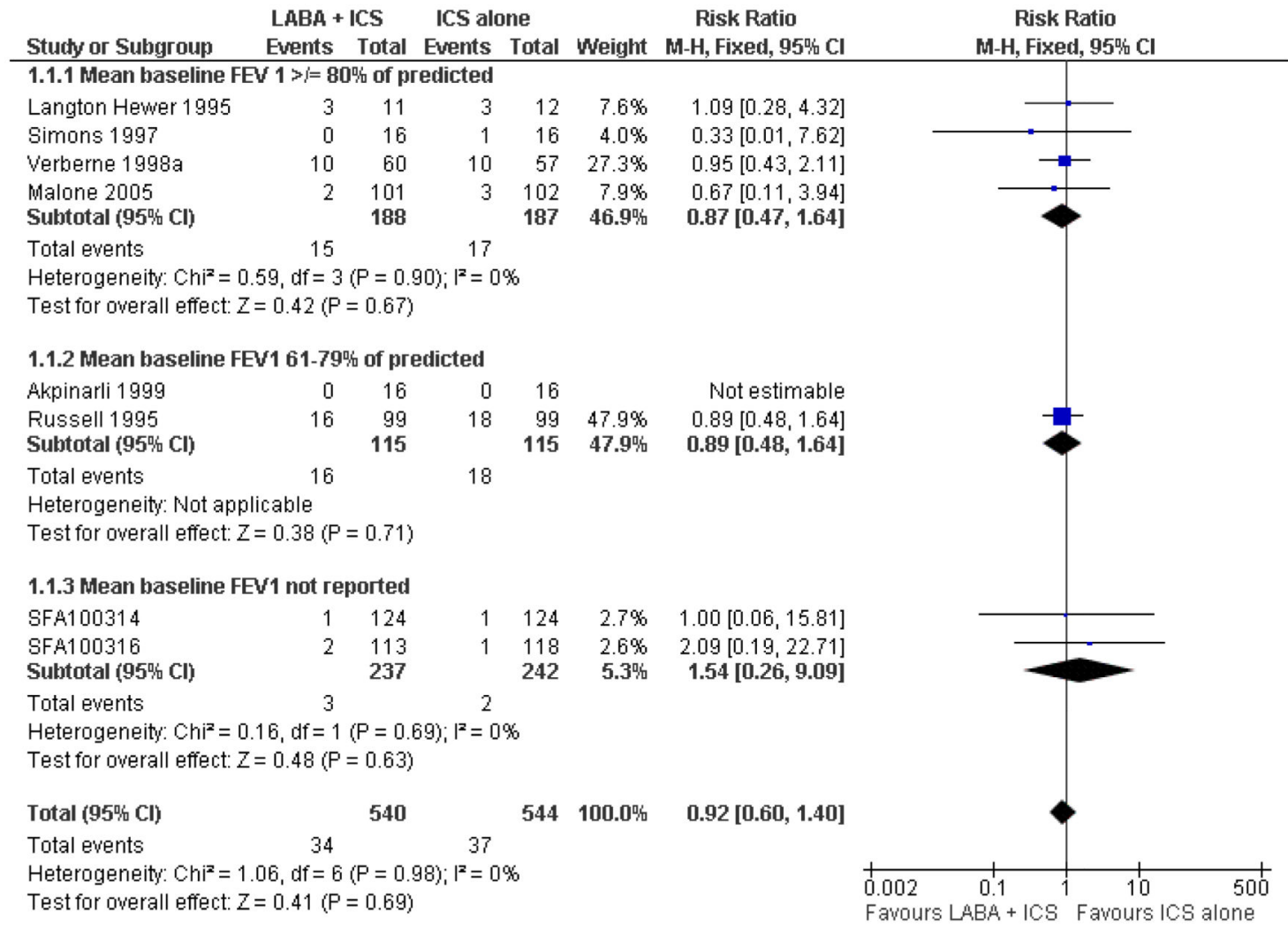

Figure 3.

Forest plot of comparison: 1 Long-acting beta 2 versus placebo: both groups receiving similar dose ICS, outcome: 1.1 \# patients with exacerbations requiring systemic steroids. 


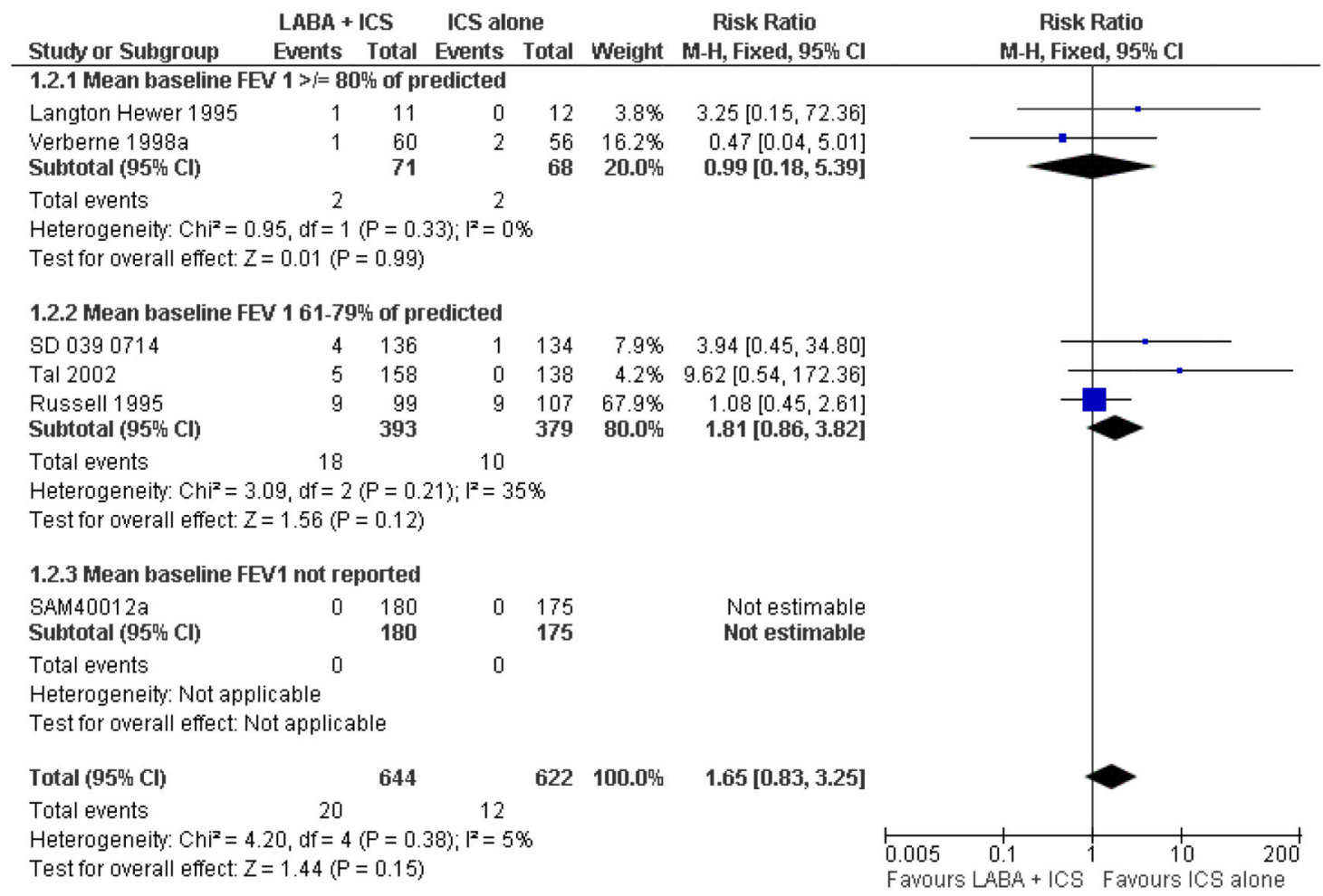

Figure 4.

Forest plot of comparison: 1 Long-acting beta2 versus placebo: both groups receiving similar dose ICS, outcome: 1.2 \# patients with exacerbations requiring hospitalisation. 


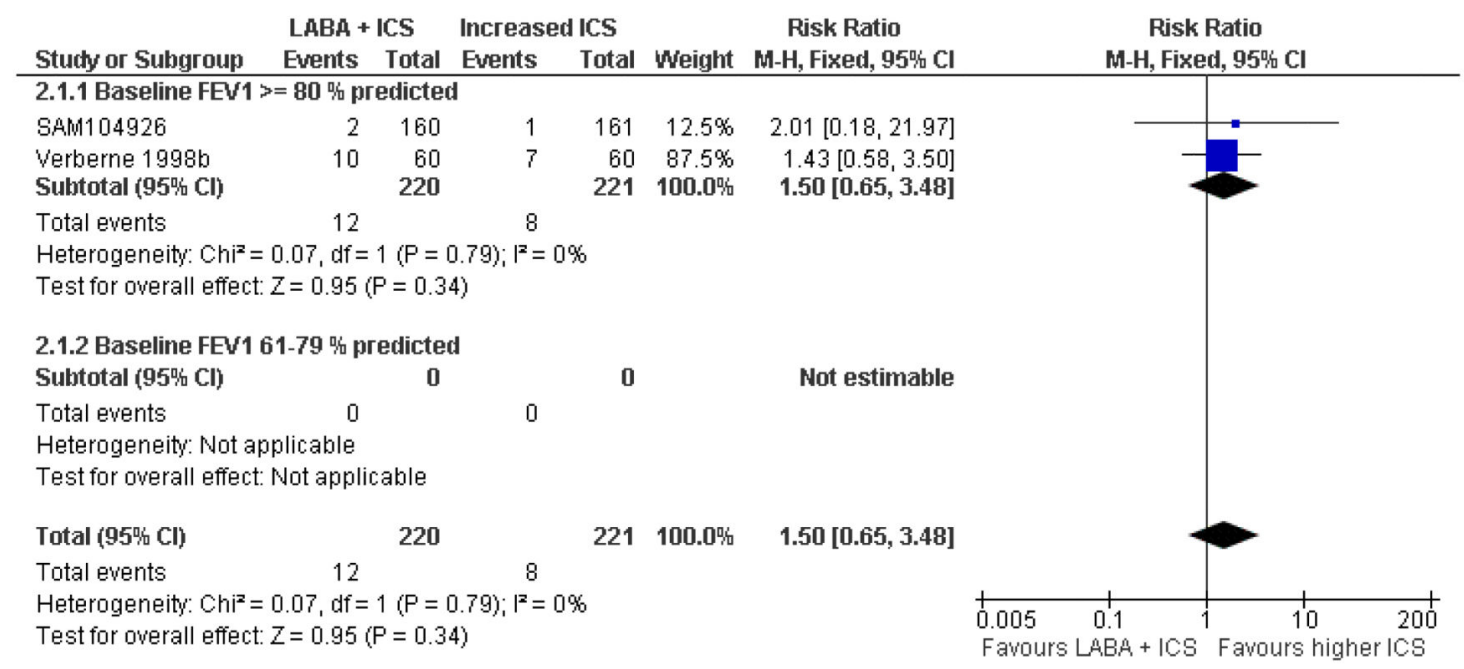

Figure 5.

Forest plot of comparison: 2 Long-acting beta2 agonist + ICS versus placebo + higher dose of ICS, outcome: 2.1 \# patients with exacerbations requiring oral steroids. 


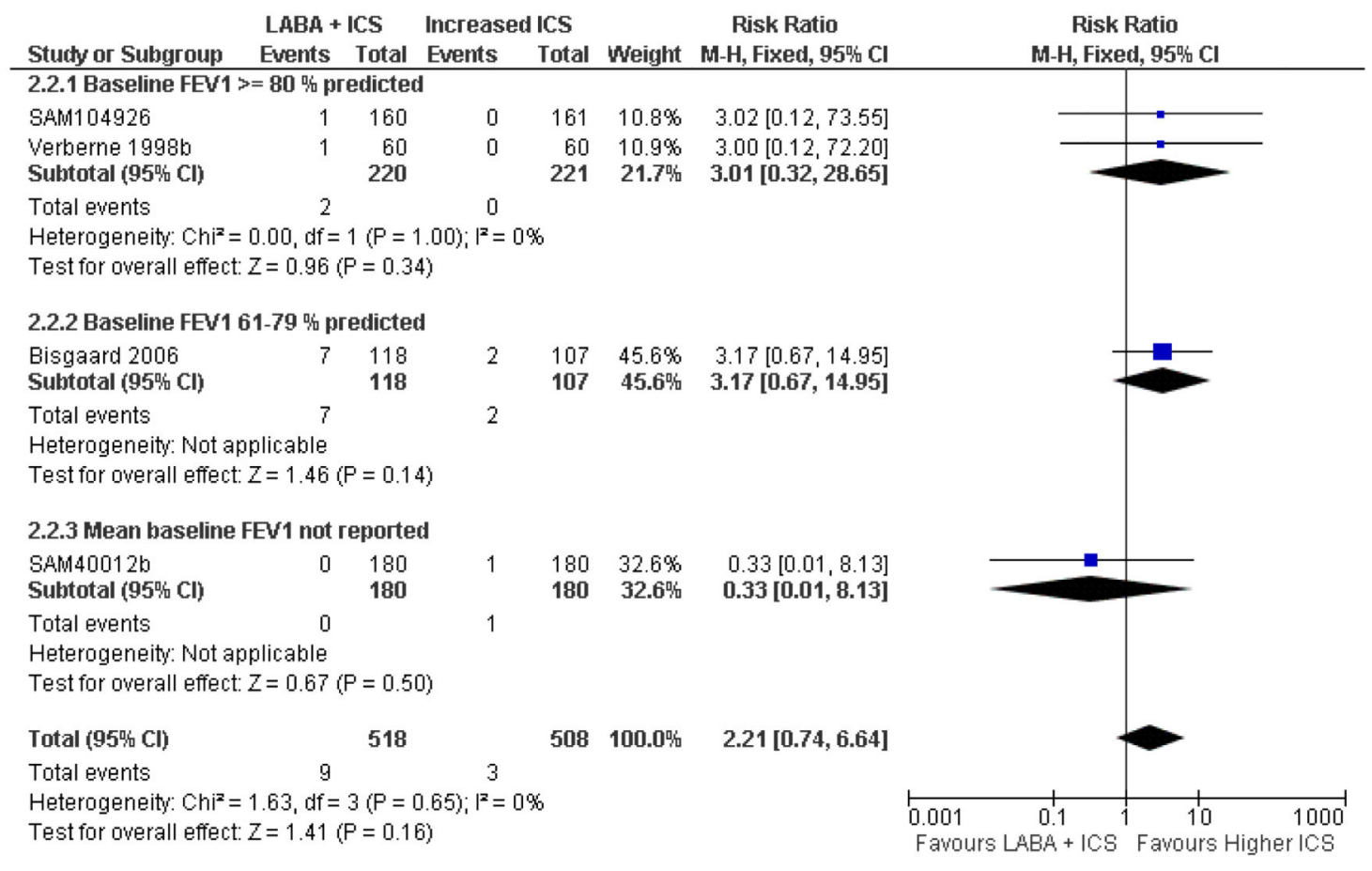

Figure 6.

Forest plot of comparison: 2 Long-acting beta2 agonist + ICS versus placebo + higher dose of ICS, outcome: 2.2 \# patients with exacerbations requiring hospitalisation. 